\title{
Highly Reactive, General and Long-Lived Catalysts for Palladium-Catalyzed Amination of Heteroaryl and Aryl Chlorides, Bromides and Iodides: Scope and Structure-Activity Relationships
}

\author{
Qilong Shen, Tokutaro Ogata and John F. Hartwig* \\ Department of Chemistry, Yale University, P.O. Box 208107, New Haven, Connecticut 06520-8107, \\ and Department of Chemistry, University of Illinois, Box 58-6, 600 South Mathews Avenue, Urbana, \\ Illinois 61801
}

\section{Supporting Information}

General Methods. Unless otherwise noted, all manipulation were conducted inside an inert atmosphere glovebox, but procedures for conducting reations without a glovebox are provided below. ${ }^{1} \mathrm{H}$ and ${ }^{13} \mathrm{C}$ NMR spectra were recorded on a Bruker DPX 400 or $500 \mathrm{MHz}$ Spectrometer and ${ }^{31} \mathrm{P}\left\{{ }^{1} \mathrm{H}\right\}$ NMR spectra were recorded on a General Electric QE $300 \mathrm{MHz}$ spectrometer with tetramethylsilane or residual protiated solvent as a reference. All ${ }^{31} \mathrm{P}\left\{{ }^{1} \mathrm{H}\right\}$ NMR chemical shifts are reported in parts per million relative to an $85 \% \mathrm{H}_{3} \mathrm{PO}_{4}$ external standard. Shifts downfield of the standard are reported as positive. Elemental analyses were performed by Atlantic Microlabs Inc., Norcross, GA or Robertson Microlab, Inc., Madison, NJ. GC and GC/MS analyses were conducted with an HP-1 methyl silicone column. CyPF-t-Bu (CyPF- $t$-Bu = 1-dicyclohexylphosphino-2-di-t-butylphosphinoethylferrocene) was obtained from Solvias AG and Strem Chemicals and used without further purification. Ethylene glycol dimethyl ether (DME, 99.9\% purity, HPLC grade) was purchased and used without further purification. All other chemicals were used as received from commercial sources.

Table S1. Optimization of Palladium-catalyzed Coupling Reaction of 3-Chloropyridine with 1-Octylamine Catalyzed by Palladium Precursors and CyPF- $t$-Bu $(1: 1) .^{a}$

\begin{tabular}{|c|c|c|c|c|c|c|}
\hline Entry & Palladium & Base & Solvent & $\begin{array}{l}\text { Loading } \\
(\text { mol \%) }\end{array}$ & $\begin{array}{c}\text { Time } \\
\text { (h) }\end{array}$ & $\begin{array}{c}\text { Conversion [\%] } \\
\left({\text { Yield })^{b}}^{\text {Con }}\right.\end{array}$ \\
\hline 1 & $\mathrm{Pd}(\mathrm{dba})_{2}$ & $\mathrm{NaOtBu}$ & DME & 1 & 10 & 100 \\
\hline 2 & $\mathrm{PdCl}_{2}(\mathrm{PhCN})_{2}$ & $\mathrm{NaOtBu}$ & DME & 1 & 10 & 96 \\
\hline 3 & $\mathrm{Pd}_{2}(\mathrm{dba})_{3}$ & $\mathrm{NaOtBu}$ & DME & 1 & 10 & 100 \\
\hline 4 & $\mathrm{Pd}(\mathrm{OAc})_{2}$ & $\mathrm{NaOtBu}$ & DME & 1 & 1 & $100(99)$ \\
\hline 5 & $\mathrm{Pd}(\mathrm{OAc})_{2}$ & $\mathrm{NaOtBu}$ & DME & 0.05 & 1 & 100 \\
\hline 6 & $\mathrm{Pd}(\mathrm{OAc})_{2}$ & $\mathrm{~K}_{3} \mathrm{PO}_{4}$ & DME & 0.05 & 24 & trace \\
\hline 7 & $\mathrm{Pd}(\mathrm{OAc})_{2}$ & $\mathrm{Cs}_{2} \mathrm{CO}_{3}$ & DME & 0.05 & 24 & NR \\
\hline 8 & $\mathrm{Pd}(\mathrm{OAc})_{2}$ & $\mathrm{~K}_{2} \mathrm{CO}_{3}$ & DME & 0.05 & 24 & NR \\
\hline 9 & $\mathrm{Pd}(\mathrm{OAc})_{2}$ & $\mathrm{NaOtBu}$ & Toluene & 0.05 & 5 & 100 \\
\hline 10 & $\mathrm{Pd}(\mathrm{OAc})_{2}$ & $\mathrm{NaOtBu}$ & THF & 0.05 & 1 & 100 \\
\hline 11 & $\mathrm{Pd}(\mathrm{OAc})_{2}$ & $\mathrm{NaOtBu}$ & 1,4-dioxane & 0.05 & 5 & 100 \\
\hline 12 & $\mathrm{Pd}(\mathrm{OAc})_{2}$ & $\mathrm{NaOtBu}$ & DME & 0.01 & 5 & $100(99)$ \\
\hline 13 & $\mathrm{Pd}(\mathrm{OAc})_{2}$ & $\mathrm{NaOtBu}$ & Toluene & 0.01 & 48 & 81 \\
\hline 14 & $\mathrm{Pd}(\mathrm{OAc})_{2}$ & $\mathrm{NaOtBu}$ & THF & 0.01 & 48 & 97 \\
\hline 15 & $\mathrm{Pd}(\mathrm{OAc})_{2}$ & $\mathrm{NaOtBu}$ & 1,4-dioxane & 0.01 & 48 & 87 \\
\hline 16 & $\mathrm{Pd}(\mathrm{OAc})_{2}$ & $\mathrm{NaOtBu}$ & DME & 0.005 & 24 & $100(93)$ \\
\hline
\end{tabular}

${ }^{a}$ All the experiments were conducted with a 1:1 ratio of metal to ligand, 1 mmol of 3-chloropyridine and 1.2 equiv 1octylamine, and 1.4 equiv base in $1.0 \mathrm{~mL}$ solvent. ${ }^{c}$ Determined by GC analysis. Isolated yields are indicated in parentheses. 
Stability of Josiphos CyPF-t-Bu. The Josiphos ligand CyPF-t-Bu (11.0 mg, $\left.2.00 \times 10^{-5} \mathrm{mmol}\right)$ was dissolved in $\mathrm{C}_{6} \mathrm{D}_{6}(0.5 \mathrm{~mL})$ in an uncapped NMR tube in air. The ${ }^{1} \mathrm{H}$ NMR and ${ }^{31} \mathrm{P}$ NMR spectra of CyPF- $t$-Bu were unchanged after $24 \mathrm{~h}$. Alternatively, CyPF- $t$-Bu $\left(11.0 \mathrm{mg}, 2.00 \times 10^{-5} \mathrm{mmol}\right)$ was weighed into an open vial in air. The solid was dissolved in $\mathrm{C}_{6} \mathrm{D}_{6}(0.5 \mathrm{~mL})$ after $24 \mathrm{~h}$. The ${ }^{1} \mathrm{H}$ NMR and ${ }^{31} \mathrm{P}$ NMR spectra of Josiphos CyPF- $t$-Bu also were unchanged.

General Procedure A for Catalytic Amination of Heteroaryl and Aryl Halides in DME. The reaction conditions and average yields for each reaction are shown in Table 1 and 2 . A typical procedure is given for the eleventh entry in Table 1 .

Stock Solution A $\left(\mathbf{1 . 0} \times \mathbf{1 0}^{-2} \mathbf{M}\right)$ : $\mathrm{DME}(1.0 \mathrm{~mL})$ was added to the mixture of $\mathrm{Pd}(\mathrm{OAc})_{2}(2.2 \mathrm{mg}, 1.0 \times$ $\left.10^{-2} \mathrm{mmol}\right)$ and CyPF- $t$-Bu $\left(5.5 \mathrm{mg}, 1.0 \times 10^{-2} \mathrm{mmol}\right)$. The resulting orange colored solution was stirred at room temperature for one minute before using.

Stock Solution B $\left(1.0 \times \mathbf{1 0}^{-4} \mathbf{M}\right): 10.0 \mu \mathrm{L}$ of the stock solution A was diluted to $1.0 \mathrm{~mL}$ with DME. The resulting pale yellow colored solution was stirred at room temperature for one minute before using.

$N$-Octyl-3-aminopyridine. (Table 1, entry 11). A solution of $\mathrm{Pd}(\mathrm{OAc})_{2}$ and $\mathrm{CyPF}-t$-Bu $(5.0 \mu \mathrm{L}$ from stock solution $\left.\mathbf{A}, 5.0 \times 10^{-5} \mathrm{mmol}\right)$ was added to a $4 \mathrm{~mL}$ vial containing 3 -chloropyridine $(0.114 \mathrm{~g}$, $1.00 \mathrm{mmol})$ and sodium tert-butoxide $(0.135 \mathrm{~g}, 1.40 \mathrm{mmol})$ in $1.0 \mathrm{~mL}$ of DME. Octylamine $(0.155 \mathrm{~g}$, $1.20 \mathrm{mmol}$ ) was then added by syringe. The vial was sealed with a cap containing a PTFE septum, and the reaction mixture was stirred at $90{ }^{\circ} \mathrm{C}$ until 3-chloropyridine was consumed, as determined by GC. The reaction solution was directly adsorbed onto silica gel, and the product was isolated by eluting with hexane/ethyl acetate $(85 / 15)$ to give $197.2 \mathrm{mg}(93 \%)$ of $N$-octyl-3-aminopyridine as a yellow solid. ${ }^{1} \mathrm{H}$ NMR $\left(\mathrm{CDCl}_{3}\right) \delta 8.02(\mathrm{~d}, J=2.8 \mathrm{~Hz}, 1 \mathrm{H}), 7.93(\mathrm{dd}, J=4.4,1.6 \mathrm{~Hz}, 1 \mathrm{H}), 7.07(\mathrm{dd}, J=8.4$, $4.6 \mathrm{~Hz}, 1 \mathrm{H}), 6.86(\mathrm{ddd}, J=8.8,3.2,1.6 \mathrm{~Hz}, 1 \mathrm{H}), 3.72(\mathrm{~s}, \mathrm{~b}, 1 \mathrm{H}), 3.11(\mathrm{t}, J=7.2 \mathrm{~Hz}, 2 \mathrm{H}), 1.63$ (quint, $J=7.2 \mathrm{~Hz}, 2 \mathrm{H}), 1.26-1.42(\mathrm{~m}, 10 \mathrm{H}), 0.89(\mathrm{t}, J=7.2 \mathrm{~Hz}, 3 \mathrm{H}) ;{ }^{13} \mathrm{C} \mathrm{NMR}\left(\mathrm{CDCl}_{3}\right) \delta 144.37$, 138.0, 135.69, 123.49, 118.01, 43.35, 31.63, 29.19, 29.16, 29.06, 26.92, 22.47, 13.91; Anal. Calcd. For $\mathrm{C}_{13} \mathrm{H}_{22} \mathrm{~N}_{2}$ : C, 75.68; H, 10.75; N, 13.58. Found: C, 75.74; H, 10.94; N, 13.79.

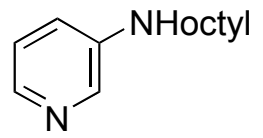

$N$-Octyl-2-aminopyridine. (Table 1, entry 1). The general procedure A conducted with 2chloropyridine $(0.114 \mathrm{~g}, 1.00 \mathrm{mmol})$, octylamine $(0.155 \mathrm{~g}, 1.20 \mathrm{mmol}), \mathrm{Pd}(\mathrm{OAc})_{2}$ and $\mathrm{CyPF}-t$-Bu $\left(100.0 \mu \mathrm{L}\right.$ from stock solution $\left.\mathbf{B}, 1.0 \times 10^{-5} \mathrm{mmol}\right)$ gave $176.3 \mathrm{mg}(86 \%)$ of $N$-benzyl-2-aminopyridine as a colorless liquid. ${ }^{1} \mathrm{H} \mathrm{NMR}\left(\mathrm{CDCl}_{3}\right) \delta 8.07(\mathrm{~d}, J=4.8 \mathrm{~Hz}, 1 \mathrm{H}), 7.42(\mathrm{dd}, J=8.6,7.2 \mathrm{~Hz}, 1 \mathrm{H}), 6.38$ $(\mathrm{d}, J=8.4 \mathrm{~Hz}, 1 \mathrm{H}), 6.55(\mathrm{dd}, J=7.0,4.8 \mathrm{~Hz}, 1 \mathrm{H}), 4.55(\mathrm{~s}, \mathrm{~b}, 1 \mathrm{H}), 3.24(\mathrm{q}, J=6.8 \mathrm{~Hz}, 2 \mathrm{H}), 1.62$ (quint, $J=7.2 \mathrm{~Hz}, 2 \mathrm{H}), 1.20-1.42(\mathrm{~m}, 10 \mathrm{H}), 0.89(\mathrm{t}, J=6.8 \mathrm{~Hz}, 3 \mathrm{H}) ;{ }^{13} \mathrm{C} \mathrm{NMR}\left(\mathrm{CDCl}_{3}\right) \delta 158.88$, 148.14, 137.19, 12.32, 106.1, 42.32, 31.68, 29.59, 29.42, 29.24, 27.11, 22.51, 13.95; Anal. Calcd. For $\mathrm{C}_{13} \mathrm{H}_{22} \mathrm{~N}_{2}$ : C, 75.68; H, 10.75; N, 13.58. Found: C, 75.71; H, 10.67; N, 13.74.

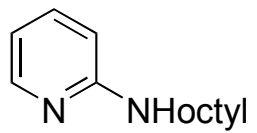

N-Octyl-2-aminopyridine. (Table 1 , entry 2). The general procedure A conducted with 2iodopyridine $(0.205 \mathrm{~g}, 1.00 \mathrm{mmol})$, octylamine $(0.155 \mathrm{~g}, 1.20 \mathrm{mmol}), \mathrm{Pd}(\mathrm{OAc})_{2}$ and CyPF-t-Bu $(5.0$ $\mu \mathrm{L}$ from stock solution $\mathbf{A}, 5.0 \times 10^{-5} \mathrm{mmol}$ ) gave $12.2 \mathrm{mg}(84 \%)$ of $N$-octyl-2-aminopyridine as a yellow solid.of $N$-octyl-2-aminopyridine as a yellow solid. $\left({ }^{1} \mathrm{H}\right.$ and ${ }^{13} \mathrm{C}$ NMR spectra are the same as those for the product in Table 1, entry 1.) 
N-Octyl-2-aminopyridine. (Table 1, entry 3). The general procedure A conducted with 2bromopyridine $(0.158 \mathrm{~g}, 1.00 \mathrm{mmol})$, octylamine $(0.155 \mathrm{~g}, 1.20 \mathrm{mmol}), \mathrm{Pd}(\mathrm{OAc})_{2}$ and CyPF-t-Bu $\left(50.0 \mu \mathrm{L}\right.$ from stock solution $\left.\mathbf{B}, 5.0 \times 10^{-6} \mathrm{mmol}\right)$ gave $196.8 \mathrm{mg}(96 \%)$ of $N$-octyl-2-aminopyridine as a yellow solid. $\left({ }^{1} \mathrm{H}\right.$ and ${ }^{13} \mathrm{C}$ NMR spectra are the same as those for the product in Table 1, entry 1.)

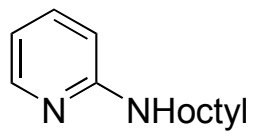

$\boldsymbol{N}$-benzyl-2-aminopyridine. ${ }^{1}$ (Table 1, entry 4). The general procedure A conducted with 2chloropyridine $(0.114 \mathrm{~g}, 1.00 \mathrm{mmol})$, benzylamine $(0.129 \mathrm{~g}, 1.20 \mathrm{mmol}), \mathrm{Pd}(\mathrm{OAc})_{2}$ and $\mathrm{CyPF}-t-\mathrm{Bu}$ $\left(100.0 \mu \mathrm{L}\right.$ from stock solution $\left.\mathbf{B}, 1.0 \times 10^{-5} \mathrm{mmol}\right)$ gave $173.6 \mathrm{mg}(85 \%)$ of $N$-benzyl-2-aminopyridine as a colorless liquid. ${ }^{1} \mathrm{H}$ NMR $\left(\mathrm{CDCl}_{3}\right) \delta 7.95(\mathrm{dt}, J=4.81 .0 \mathrm{~Hz}, 1 \mathrm{H}), 7.26(\mathrm{td}, J=8.0,1.2 \mathrm{~Hz}, 1 \mathrm{H})$, $7.24(\mathrm{~d}, J=8.0 \mathrm{~Hz}, 2 \mathrm{H}), 7.21(\mathrm{t}, J=8.0 \mathrm{~Hz}, 2 \mathrm{H}), 7.15(\mathrm{t}, J=7.2 \mathrm{~Hz}, 1 \mathrm{H}), 6.45(\mathrm{dd}, J=7.2,4.8 \mathrm{~Hz}, 1$ $\mathrm{H}), 6.23(\mathrm{dd}, J=8.0,0.8 \mathrm{~Hz}, 1 \mathrm{H}), 5.13(\mathrm{~s}, 1 \mathrm{H}), 4.38(\mathrm{~d}, J=5.6 \mathrm{~Hz}, 2 \mathrm{H}) ;{ }^{13} \mathrm{C} \mathrm{NMR}\left(\mathrm{CDCl}_{3}\right) \delta 158.63$, $148.08,139.14,137.31,128.49,127.28,127.06,112.92,106.60,46.17$.<smiles>Brc1ccccn1</smiles>

$N$-Benzyl-2-aminopyridine. ${ }^{1}$ (Table 1, entry 5). The general procedure A conducted with 2bromopyridine $(0.158 \mathrm{~g}, 1.00 \mathrm{mmol})$, benzylamine $(0.129 \mathrm{~g}, 1.20 \mathrm{mmol}), \mathrm{Pd}(\mathrm{OAc})_{2}$ and CyPF- $t$-Bu $\left(50.0 \mu \mathrm{L}\right.$ from stock solution $\left.\mathbf{B}, 5.0 \times 10^{-6} \mathrm{mmol}\right)$ gave $152.7 \mathrm{mg}(83 \%)$ of $N$-benzyl-2-aminopyridine as a white solid. $\left({ }^{1} \mathrm{H}\right.$ and ${ }^{13} \mathrm{C}$ NMR spectra are the same as those for the product in Table 1 , entry 4 .)<smiles>Brc1ccccn1</smiles>

$\mathbf{N}$-Cyclohexyl-2-aminopyridine. ${ }^{1}$ (Table 1, entry 6). The general procedure A conducted with 2chloropyridine $(0.114 \mathrm{~g}, 1.00 \mathrm{mmol})$, cyclohexylamine $(0.119 \mathrm{~g}, 1.20 \mathrm{mmol}), \mathrm{Pd}(\mathrm{OAc})_{2}$ and CyPF-t$\mathrm{Bu}\left(10.0 \mu \mathrm{L}\right.$ from stock solution $\left.\mathbf{A}, 1.0 \times 10^{-4} \mathrm{mmol}\right)$ gave $173.1 \mathrm{mg}(98 \%)$ of $N$-cyclonexyl-2aminopyridine as a white solid. ${ }^{1} \mathrm{H} \mathrm{NMR}\left(\mathrm{CDCl}_{3}\right) \delta 7.97(\mathrm{dt}, J=5.0,0.8 \mathrm{~Hz}, 1 \mathrm{H}), 7.28(\mathrm{ddd}, J=8.4$, 6.8, $1.6 \mathrm{~Hz}, 1 \mathrm{H}), 6.42(\mathrm{dd}, J=6.8,5.0 \mathrm{~Hz}, 1 \mathrm{H}), 6.26(\mathrm{~d}, J=8.0 \mathrm{~Hz}, 1 \mathrm{H}), 4.48(\mathrm{~d}, J=6.4 \mathrm{~Hz}, 1 \mathrm{H})$, 3.41-3.49 (m, $1 \mathrm{H}), 1.93-1.97(\mathrm{~m}, 2 \mathrm{H}), 1.66(\mathrm{dt}, J=13.2,3.6 \mathrm{~Hz}, 2 \mathrm{H}), 1.55$ (dt, $J=12.4,4.0 \mathrm{~Hz}, 1$ $\mathrm{H}), 1.25-1.36(\mathrm{~m}, 2 \mathrm{H}), 1.06-1.19(\mathrm{~m}, 3 \mathrm{H}) ;{ }^{13} \mathrm{C} \mathrm{NMR}\left(\mathrm{CDCl}_{3}\right) \delta 158.03,148.13,137.11,112.14$, $106.57,49.95,33.23,25.67,24.78$.<smiles>[Al]Nc1ccccn1</smiles>

$\mathbf{N}$-Cyclohexyl-2-aminopyridine. ${ }^{1}$ (Table 1, entry 7). The general procedure A conducted with 2bromopyridine $(0.158 \mathrm{~g}, 1.00 \mathrm{mmol})$, cyclohexylamine $(0.119 \mathrm{~g}, 1.20 \mathrm{mmol}), \mathrm{Pd}(\mathrm{OAc})_{2}$ and CyPF- $t$ $\mathrm{Bu}\left(5.0 \mu \mathrm{L}\right.$ from stock solution $\left.\mathbf{A}, 5.0 \times 10^{-5} \mathrm{mmol}\right)$ gave $168.4 \mathrm{mg}(96 \%)$ of $N$-cyclonexyl-2- 
aminopyridine as a white solid. $\left({ }^{1} \mathrm{H}\right.$ and ${ }^{13} \mathrm{C}$ NMR spectra are the same as those for the product in Table 2, entry 4.)

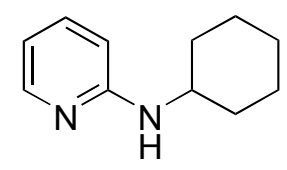

N-sec-Butyl-2-aminopyridine. (Table 1, entry 8). The general procedure conducted with 2iodopyridine $(0.125 \mathrm{~g}, 1.00 \mathrm{mmol})$, sec-butylamine $(87.8 \mathrm{mg}, 1.20 \mathrm{mmol}), \mathrm{Pd}(\mathrm{OAc})_{2}$ and CyPF-t-Bu $\left(5.0 \mu \mathrm{L}\right.$ from stock solution $\left.\mathbf{A}, 5.0 \times 10^{-5} \mathrm{mmol}\right)$ and sodium tert-butoxide $(0.135 \mathrm{~g}, 1.40 \mathrm{mmol})$ gave $146.8 \mathrm{mg}(98 \%)$ of $\mathrm{N}$-sec-butyl-2-aminopyridine as a colorless liquid. ${ }^{1} \mathrm{H} \mathrm{NMR}\left(\mathrm{CDCl}_{3}\right) \delta 7.98(\mathrm{dt}, J=$ $5.0,0.8 \mathrm{~Hz}, 1 \mathrm{H}), 7.30(\mathrm{ddd}, J=8.8,7.6,2.0 \mathrm{~Hz}, 1 \mathrm{H}), 6.43(\mathrm{ddd}, J=7.2,4.8,0.8 \mathrm{~Hz}, 1 \mathrm{H}), 6.26(\mathrm{~d}, J$ $=8.4 \mathrm{~Hz}, 1 \mathrm{H}), 4.37(\mathrm{~d}, J=4.8 \mathrm{~Hz}, 1 \mathrm{H}), 3.57-3.62(\mathrm{~m}, 1 \mathrm{H}), 1.42-1.52(\mathrm{~m}, 2 \mathrm{H}), 1.11(\mathrm{~d}, J=6.4 \mathrm{~Hz}, 3$ $\mathrm{H}), 0.87(\mathrm{t}, J=7.6 \mathrm{~Hz}, 3 \mathrm{H}) ;{ }^{13} \mathrm{C} \mathrm{NMR}\left(\mathrm{CDCl}_{3}\right) \delta 158.37,148.17,137.23,112.20,106.52,48.38$, 29.69, 20.28, 10.27. Anal. Calcd. For $\mathrm{C}_{9} \mathrm{H}_{14} \mathrm{~N}_{2}$ : C, 71.96; H, 9.39; N, 18.65. Found: C, 72.01; H, 9.58; $\mathrm{N}, 18.51$.

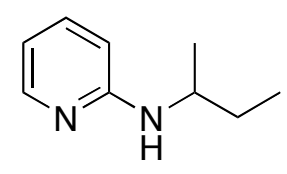

$\boldsymbol{N}$-(2-Pyridyl)- $\alpha$-methyl-benzylamine. (Table 1, entry 9). ). The general procedure A conducted with 2-chloropyridine $(0.114 \mathrm{~g}, 1.00 \mathrm{mmol}), \alpha$-methyl-benzylamine $(0.145 \mathrm{~g}, 1.20 \mathrm{mmol}, 99 \% \mathrm{ee})$, $\mathrm{Pd}(\mathrm{OAc})_{2}$ and $\mathrm{CyPF}-t$-Bu $\left(50.0 \mu \mathrm{L}\right.$ from stock solution $\left.\mathbf{A}, 5.0 \times 10^{-4} \mathrm{mmol}\right)$ gave $181.3 \mathrm{mg}(91 \%$, $94.5 \%$ ee) of $N$-(2-pyridyl)- $\alpha$-methyl-benzylamine. The ee was determined on Chiralcel OD-H column with hexane $/ 2$-propanol $=90 / 10$, flow $=1.0 \mathrm{ml} / \mathrm{min}$, wavelength $=254 \mathrm{~nm}$. Retention time $5.71 \mathrm{mins}$ (S-isomer), $9.28 \mathrm{mins}$ (R-isomer). ${ }^{1} \mathrm{H} \mathrm{NMR}\left(\mathrm{CDCl}_{3}\right) \delta 7.97$ (ddd, $\left.J=5.0,2.0,1.0 \mathrm{~Hz}, 1 \mathrm{H}\right), 7.28(\mathrm{dd}, J$ $=7.5,1.0 \mathrm{~Hz}, 2 \mathrm{H}), 7.22(\mathrm{~d}, J=7.5 \mathrm{~Hz}, 1 \mathrm{H}), 7.17-7.21(\mathrm{~m}, 2 \mathrm{H}), 7.12(\mathrm{t}, J=7.0 \mathrm{~Hz}, 1 \mathrm{H}), 6.42(\mathrm{ddd}, J$ $=7.5,5.0,1.0 \mathrm{~Hz}, 1 \mathrm{H}), 6.08(\mathrm{~d}, J=8.0 \mathrm{~Hz}, 1 \mathrm{H}), 5.19(\mathrm{~d}, J=5.5 \mathrm{~Hz}, 1 \mathrm{H}), 4.62$ (quint, $J=6.5 \mathrm{~Hz}, 1$ $\mathrm{H}), 1.44(\mathrm{~d}, J=6.5 \mathrm{~Hz}, 3 \mathrm{H}) ;{ }^{13} \mathrm{C} \mathrm{NMR}\left(\mathrm{CDCl}_{3}\right) 158.03,148.08,144.63,137.33,128.52,126.87$, 127.45, 112.87, 106.51, 51.81, 24.30. Anal. Calcd. For $\mathrm{C}_{13} \mathrm{H}_{14} \mathrm{~N}_{2}$ : C, 78.75; H, 7.12; N, 14.13. Found: C, 78.67; H, 7.11; N, 14.13.

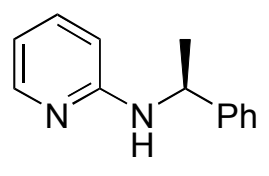

N-Octyl-2-amino-3-methylpyridine. (Table 1, entry 10). The general procedure A conducted with 2chloro-3-methylpyridine $(0.126 \mathrm{~g}, 1.00 \mathrm{mmol})$, octylamine $(0.155 \mathrm{~g}, 1.20 \mathrm{mmol}), \mathrm{Pd}(\mathrm{OAc})_{2}$ and CyPF$t$-Bu $\left(5.0 \mu \mathrm{L}\right.$ from stock solution $\left.\mathbf{A}, 5.0 \times 10^{-5} \mathrm{mmol}\right)$ gave $211.6 \mathrm{mg}(96 \%)$ of $N$-octyl-2-amino-3methylpyridine as a pale yellow liquid. ${ }^{1} \mathrm{H} \mathrm{NMR}\left(\mathrm{CDCl}_{3}\right) \delta 7.98(\mathrm{~d}, J=4.2 \mathrm{~Hz}, 1 \mathrm{H}), 7.14(\mathrm{~d}, J=7.2$ $\mathrm{Hz}, 1 \mathrm{H}), 6.44(\mathrm{dd}, J=6.8,5.2 \mathrm{~Hz}, 1 \mathrm{H}), 4.03(\mathrm{~s}, \mathrm{~b}, 1 \mathrm{H}), 3.41(\mathrm{q}, J=6.5 \mathrm{~Hz}, 2 \mathrm{H}), 2.02(\mathrm{~s}, 3 \mathrm{H}), 1.60$ (quint, $J=7.2 \mathrm{~Hz}, 2 \mathrm{H}), 1.19-1.40(\mathrm{~m}, 10 \mathrm{H}), 0.84(\mathrm{t}, J=6.8 \mathrm{~Hz}, 3 \mathrm{H}) ;{ }^{13} \mathrm{C} \mathrm{NMR}\left(\mathrm{CDCl}_{3}\right) \delta 156.90$, 145.38, 136.44, 116.18, 112.10, 41.68, 31.75, 29.81, 29.35, 29.19, 27.11, 22.57, 16.86, 14.00. Anal. Calcd. For $\mathrm{C}_{14} \mathrm{H}_{24} \mathrm{~N}_{2}$ : C, 76.31; H, 10.98; N, 12.71. Found: C, 76.25; H, 10.99; N, 12.41. 
$\mathrm{N}$-Octyl-3-aminopyridine. (Table 1, entry 11). The general procedure A conducted with 3chloropyridine ( $0.114 \mathrm{~g}, 1.00 \mathrm{mmol})$, octylamine $(0.155 \mathrm{~g}, 1.20 \mathrm{mmol}), \mathrm{Pd}(\mathrm{OAc})_{2}$ and $\mathrm{CyPF}-t-\mathrm{Bu}(5.0$ $\mu \mathrm{L}$ from stock solution A, $5.0 \times 10^{-5} \mathrm{mmol}$ ) gave $197.2 \mathrm{mg}(93 \%)$ of $N$-octyl-3-aminopyridine as a yellow solid. ${ }^{1} \mathrm{H}$ NMR $\left(\mathrm{CDCl}_{3}\right) \delta 8.02(\mathrm{~d}, J=2.8 \mathrm{~Hz}, 1 \mathrm{H}), 7.93(\mathrm{dd}, J=4.4,1.6 \mathrm{~Hz}, 1 \mathrm{H}), 7.07(\mathrm{dd}, J$ $=8.4,4.6 \mathrm{~Hz}, 1 \mathrm{H}), 6.86(\mathrm{ddd}, J=8.8,3.2,1.6 \mathrm{~Hz}, 1 \mathrm{H}), 3.72(\mathrm{~s}, \mathrm{~b}, 1 \mathrm{H}), 3.11(\mathrm{t}, J=7.2 \mathrm{~Hz}, 2 \mathrm{H}), 1.63$ (quint, $J=7.2 \mathrm{~Hz}, 2 \mathrm{H}), 1.26-1.42(\mathrm{~m}, 10 \mathrm{H}), 0.89(\mathrm{t}, J=7.2 \mathrm{~Hz}, 3 \mathrm{H}) ;{ }^{13} \mathrm{C}$ NMR $\left(\mathrm{CDCl}_{3}\right) \delta 144.37$, 138.0, 135.69, 123.49, 118.01, 43.35, 31.63, 29.19, 29.16, 29.06, 26.92, 22.47, 13.91; Anal. Calcd. For $\mathrm{C}_{13} \mathrm{H}_{22} \mathrm{~N}_{2}: \mathrm{C}, 75.68 ; \mathrm{H}, 10.75 ; \mathrm{N}, 13.58$. Found: C, 75.74; H, 10.94; N, 13.79 .

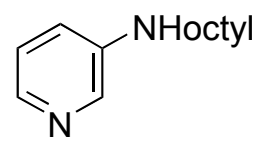

$\mathrm{N}$-Octyl-3-aminopyridine. (Table 1, entry 12). The following procedure was performed without a drybox. An oven-dried resealable Schlenk flask capped with a rubber septum was evacuated and backfilled with $\mathrm{N}_{2}$. To the flask was added $\mathrm{NaO} t \mathrm{Bu}(0.135 \mathrm{~g}, 1.40 \mathrm{mmol})$ and a stir bar. The flask was evacuated and backfilled with $\mathrm{N}_{2}$ three times. To the flask was then added 3-chloropyridine $(0.114 \mathrm{~g}$, $1.00 \mathrm{mmol}, 95.0 \mu \mathrm{L}), \mathrm{DME}(1.0 \mathrm{~mL})$, a stock solution $(5.0 \mu \mathrm{L})$ containing $\mathrm{Pd}(\mathrm{OAc})_{2}\left(5.0 \times 10^{-5} \mathrm{mmol}\right)$ and CyPF- $t$-Bu $\left(5.0 \times 10^{-5} \mathrm{mmol}\right)$ and octylamine $(0.155 \mathrm{~g}, 1.20 \mathrm{mmol})$. The rubber septum was wrapped with vinyl electrical tape to prevent leaking. The resulting mixture was stirred for $48 \mathrm{~h}$ at 100 ${ }^{\circ} \mathrm{C}$ until the 3-chloropyridine was consumed, as determined by GC. The reaction solution was directly adsorbed onto silica gel, and the product was isolated by eluting with hexane/ethyl acetate (85/15) to give $204.3 \mathrm{mg}$ (99\%) of $\mathrm{N}$-octyl-3-aminopyridine as a yellow solid. $\left({ }^{1} \mathrm{H}\right.$ and ${ }^{13} \mathrm{C}$ NMR spectra are the same as those for the product in Table 1, entry 11.)<smiles>[Mg]ONc1cccnc1</smiles>

$\mathrm{N}$-octyl-3-aminopyridine. (Table 1, entry 13). The general procedure A conducted with 3bromopyridine $(0.158 \mathrm{~g}, 1.00 \mathrm{mmol})$, octylamine $(0.155 \mathrm{~g}, 1.20 \mathrm{mmol}), \mathrm{Pd}(\mathrm{OAc})_{2}$ and $\mathrm{CyPF}-t-\mathrm{Bu}(5.0$ $\mu \mathrm{L}$ from stock solution A, $5.0 \times 10^{-5} \mathrm{mmol}$ ) gave $207.7 \mathrm{mg}(99 \%)$ of $N$-octyl-3-aminopyridine as a white solid. $\left({ }^{1} \mathrm{H}\right.$ and ${ }^{13} \mathrm{C}$ NMR spectra are the same as those for the product in Table 1, entry 11.)

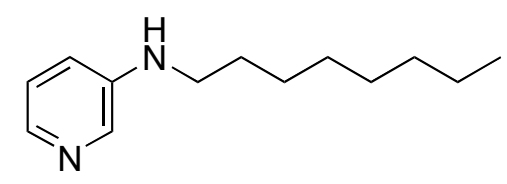

$\mathrm{N}$-Benzyl-3-aminopyridine. ${ }^{2}$ (Table 1, entry 14). The general procedure A conducted with 3chloropyridine $(0.114 \mathrm{~g}, 1.00 \mathrm{mmol})$, benzylamine $(0.129 \mathrm{~g}, 1.20 \mathrm{mmol}), \mathrm{Pd}(\mathrm{OAc})_{2}$ and $\mathrm{CyPF}-t-\mathrm{Bu}$ (10.0 $\mu \mathrm{L}$ from stock solution $\left.\mathbf{A}, 1.0 \times 10^{-4} \mathrm{mmol}\right)$ gave $174.8 \mathrm{mg}(95 \%)$ of $N$-benzyl-3-aminopyridine as a yellow solid. ${ }^{1} \mathrm{H}$ NMR $\left(\mathrm{CDCl}_{3}\right) \delta 7.93(\mathrm{~d}, J=2.8 \mathrm{~Hz}, 1 \mathrm{H}), 7.83(\mathrm{dd}, J=4.8,1.6 \mathrm{~Hz}, 1 \mathrm{H}), 7.23(\mathrm{~d}$, $J=4.4 \mathrm{~Hz}, 4 \mathrm{H}), 7.14-7.19(\mathrm{~m}, 1 \mathrm{H}), 6.91(\mathrm{dd}, J=8.0,4.4 \mathrm{~Hz}, 1 \mathrm{H}), 6.72(\mathrm{ddd}, J=8.4,2.8,1.2 \mathrm{~Hz}, 1$ $\mathrm{H}), 4.37(\mathrm{~s}, \mathrm{~b}, 1 \mathrm{H}), 4.19(\mathrm{~d}, J=6.0 \mathrm{~Hz}, 2 \mathrm{H}) ;{ }^{13} \mathrm{C} \mathrm{NMR}\left(\mathrm{CDCl}_{3}\right) \delta 143.97,138.51,138.45,135.97$, $128.54,127.23,127.19,123.52,118.26,47.54$. 
$N$-Benzyl-3-aminopyridine. ${ }^{2}$ (Table 1 , entry 15). The general procedure A conducted with 3bromopyridine $(0.158 \mathrm{~g}, 1.00 \mathrm{mmol})$, benzylamine $(0.129 \mathrm{~g}, 1.20 \mathrm{mmol}), \mathrm{Pd}(\mathrm{OAc})_{2}$ and CyPF- $t$-Bu $\left(5.0 \mu \mathrm{L}\right.$ from stock solution $\left.\mathbf{A}, 5.0 \times 10^{-5} \mathrm{mmol}\right)$ gave $183.3 \mathrm{mg}(99 \%)$ of $N$-benzyl-3-aminopyridine as a white solid. $\left({ }^{1} \mathrm{H}\right.$ and ${ }^{13} \mathrm{C}$ NMR spectra are the same as those for the product in Table 1, entry 14.)

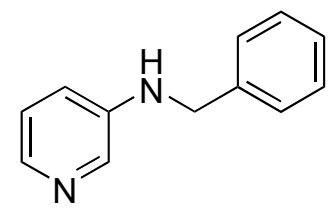

N-sec-Butyl-3-aminopyridine. (Table 1, entry 16). The general procedure A conducted with 3bromopyridine $(0.158 \mathrm{~g}, 1.00 \mathrm{mmol})$, sec-butylamine $(0.119 \mathrm{~g}, 1.20 \mathrm{mmol}), \mathrm{Pd}(\mathrm{OAc})_{2}$ and CyPF- $t$-Bu $\left(5.0 \mu \mathrm{L}\right.$ from stock solution $\left.\mathbf{A}, 5.0 \times 10^{-5} \mathrm{mmol}\right)$ gave $139.3 \mathrm{mg}(93 \%)$ of $N$-sec-butyl-3-aminopyridine as a white solid. ${ }^{1} \mathrm{H}$ NMR $\left(\mathrm{CDCl}_{3}\right) \delta 7.91(\mathrm{~d}, J=2.8 \mathrm{~Hz}, 1 \mathrm{H}), 7.80(\mathrm{dd}, J=4.4,1.2 \mathrm{~Hz}, 1 \mathrm{H}), 6.95$ (ddd, $J=8.0,4.4,0.8 \mathrm{~Hz}, 1 \mathrm{H}), 6.73(\mathrm{ddd}, J=8.4,2.8,1.4 \mathrm{~Hz}, 1 \mathrm{H}), 3.74$ (s, b, $1 \mathrm{H}), 3.28-3.31$ (m, 1 $\mathrm{H}), 1.44-1.55(\mathrm{~m}, 1 \mathrm{H}), 1.33-1.42(\mathrm{~m}, 1 \mathrm{H}), 1.07(\mathrm{~d}, J=6.4 \mathrm{~Hz}, 3 \mathrm{H}), 0.85(\mathrm{t}, J=7.6 \mathrm{~Hz}, 3 \mathrm{H}) ;{ }^{13} \mathrm{C}$ NMR $\left(\mathrm{CDCl}_{3}\right) \delta 143.61,137.62,135.99,123.48,118.32,49.30,29.18,19.76,10.08$; Anal. Calcd. For $\mathrm{C}_{9} \mathrm{H}_{14} \mathrm{~N}_{2}$ : C, 71.96; H, 9.39; N, 18.65. Found: C, 71.69; H, 9.41; N, 18.65.<smiles>CCC(C)Nc1cccnc1</smiles>

N-iso-Butyl-2-aminopyridine. (Table 1, entry 17). The general procedure conducted with 3iodopyridine $(0.125 \mathrm{~g}, 1.00 \mathrm{mmol})$, iso-butylamine $(87.8 \mathrm{mg}, 1.20 \mathrm{mmol}), \mathrm{Pd}(\mathrm{OAc})_{2}$ and $\mathrm{CyPF}-t$-Bu $\left(50.0 \mu \mathrm{L}\right.$ from stock solution $\left.\mathbf{A}, 5.0 \times 10^{-4} \mathrm{mmol}\right)$ and sodium tert-butoxide $(0.135 \mathrm{~g}, 1.40 \mathrm{mmol})$ gave $144.0 \mathrm{mg}(96 \%)$ of $\mathrm{N}$-iso-butyl-2-aminopyridine as a colorless liquid. ${ }^{1} \mathrm{H} \mathrm{NMR}\left(\mathrm{CDCl}_{3}\right) \delta 7.93(\mathrm{~d}, J=$ $2.8 \mathrm{~Hz}, 1 \mathrm{H}), 7.82(\mathrm{dd}, J=4.8,1.2 \mathrm{~Hz}, 1 \mathrm{H}), 6.95(\mathrm{dd}, J=8.4,4.4 \mathrm{~Hz}, 1 \mathrm{H}), 6.75(\mathrm{ddd}, J=8.0,2.8,1.2$ $\mathrm{Hz}, 1 \mathrm{H}), 4.01(\mathrm{~s}, 1 \mathrm{H}), 2.82(\mathrm{t}, J=4.8 \mathrm{~Hz}, 2 \mathrm{H}), 1.73-1.84(\mathrm{~m}, 1 \mathrm{H}), 0.89(\mathrm{~d}, J=6.7 \mathrm{~Hz}, 6 \mathrm{H}) ;{ }^{13} \mathrm{C}$ NMR $\left(\mathrm{CDCl}_{3}\right) \delta 144.44,137.86,135.67,123.47,117.90,51.06,27.74,20.18$. Anal. Calcd. For $\mathrm{C}_{9} \mathrm{H}_{14} \mathrm{~N}_{2}$ : C, 71.96; H, 9.39; N, 18.65. Found: C, 71.88; H, 9.42; N, 18.54.

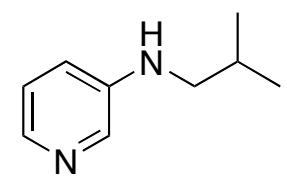

N-Cyclohexyl-3-aminopyridine. ${ }^{1}$ (Table 1, entry 18). The general procedure A conducted with 3chloropyridine $(0.114 \mathrm{~g}, 1.00 \mathrm{mmol})$, cyclohexylamine $(0.119 \mathrm{~g}, 1.20 \mathrm{mmol}), \mathrm{Pd}(\mathrm{OAc})_{2}$ and CyPF- $t$ $\mathrm{Bu}\left(10.0 \mu \mathrm{L}\right.$ from stock solution $\left.\mathbf{A}, 1.0 \times 10^{-4} \mathrm{mmol}\right)$ gave $138.4 \mathrm{mg}(79 \%)$ of $N$-cyclohexyl-3aminopyridine as a white solid. ${ }^{1} \mathrm{H} \mathrm{NMR}\left(\mathrm{CDCl}_{3}\right) \delta 7.91(\mathrm{~d}, J=2.4 \mathrm{~Hz}, 1 \mathrm{H}), 7.81(\mathrm{dd}, J=4.8,1.4 \mathrm{~Hz}$, $1 \mathrm{H}), 6.95(\mathrm{dd}, J=8.0,4.8 \mathrm{~Hz}, 1 \mathrm{H}), 6.75(\mathrm{ddd}, J=8.0,2.8,1.2 \mathrm{~Hz}, 1 \mathrm{H}), 3.73$ (s, b, $1 \mathrm{H}), 3.10-3.21$ $(\mathrm{m}, 1 \mathrm{H}), 1.94(\mathrm{dd}, J=13.2,3.2 \mathrm{~Hz}, 2 \mathrm{H}), 1.67(\mathrm{dt}, J=13.2,3.6 \mathrm{~Hz}, 2 \mathrm{H}), 1.56(\mathrm{dd}, J=12.4,3.6 \mathrm{~Hz}, 1$ $\mathrm{H}), 1.22-1.33(\mathrm{~m}, 2 \mathrm{H}), 1.02-1.18(\mathrm{~m}, 3 \mathrm{H}) ;{ }^{13} \mathrm{C} \mathrm{NMR}\left(\mathrm{CDCl}_{3}\right) \delta 143.25,137.77,136.09,123.48$, $118.39,51.16,32.94,25.59,24.68$. 
<smiles>[AlH2]Nc1cccnc1</smiles>

$\mathrm{N}$-Cyclohexyl-3-aminopyridine. ${ }^{1}$ (Table 1, entry 19). The general procedure conducted with 3iodopyridine $(0.205 \mathrm{~g}, 1.00 \mathrm{mmol})$, cyclohexylamine $(0.119 \mathrm{~g}, 1.20 \mathrm{mmol}), \mathrm{Pd}(\mathrm{OAc})_{2}$ and CyPF- $t$-Bu $\left(50.0 \mu \mathrm{L}\right.$ from stock solution $\left.\mathbf{A}, 5.0 \times 10^{-4} \mathrm{mmol}\right)$ and sodium tert-butoxide $(0.135 \mathrm{~g}, 1.40 \mathrm{mmol})$ gave $137.5 \mathrm{mg}$ (78\%) of $\mathrm{N}$-cyclohexyl-3-aminopyridine as a white solid. $\left({ }^{1} \mathrm{H}\right.$ and ${ }^{13} \mathrm{C}$ NMR spectra are the same as those for the product in Table 1, entry 18.)<smiles>[AlH2]Nc1cccnc1</smiles>

$\mathrm{N}$-tert-Butyl-3-aminopyridine. ${ }^{3}$ (Table 1, entry 20). The general procedure A conducted with 3chloropyridine ( $0.114 \mathrm{~g}, 1.00 \mathrm{mmol})$, tert-butylamine ( $87.8 \mathrm{mg}, 1.20 \mathrm{mmol}), \mathrm{Pd}(\mathrm{OAc})_{2}(2.2 \mathrm{mg}, 1.0 \times$ $\left.10^{-2} \mathrm{mmol}\right)$ and CyPF-t-Bu $\left(5.5 \mathrm{mg}, 1.0 \times 10^{-2} \mathrm{mmol}\right)$ gave $100.3 \mathrm{mg}(67 \%)$ of $N$-tert-butyl-3aminopyridine as a colorless liquid. ${ }^{1} \mathrm{H}$ NMR $\left(\mathrm{CDCl}_{3}\right) \delta 8.03(\mathrm{~s}, 1 \mathrm{H}), 7.91(\mathrm{t}, J=3.0 \mathrm{~Hz}, 1 \mathrm{H}), 6.98(\mathrm{~d}$, $J=3.2 \mathrm{~Hz}, 1 \mathrm{H}), 6.97(\mathrm{~d}, J=3.6 \mathrm{~Hz}, 1 \mathrm{H}), 3.55(\mathrm{~s}, \mathrm{~b}, 1 \mathrm{H}), 1.27(\mathrm{~s}, 9 \mathrm{H}) ;{ }^{13} \mathrm{C} \mathrm{NMR}\left(\mathrm{CDCl}_{3}\right) \delta 142.89$, $139.32,139.03,123.21,122.42,51.36,29.66$.<smiles>CC(C)(C)Nc1cccnc1</smiles>

$N$-(3-Pyridyl)- $\alpha$-methyl-benzylamine. (Table 1, entry 21). ). The general procedure A conducted with 3-chloropyridine $(0.114 \mathrm{~g}, 1.00 \mathrm{mmol})$, $\alpha$-methyl-benzylamine $(0.145 \mathrm{~g}, 1.20 \mathrm{mmol}, 99 \% \mathrm{ee})$, $\mathrm{Pd}(\mathrm{OAc})_{2}$ and $\mathrm{CyPF}-t$-Bu $\left(50.0 \mu \mathrm{L}\right.$ from stock solution $\left.\mathbf{A}, 5.0 \times 10^{-4} \mathrm{mmol}\right)$ gave $181.3 \mathrm{mg}(91 \%$, $94.5 \%$ ee) of $N$-(3-pyridyl)- $\alpha$-methyl-benzylamine. The ee was determined on Chiralcel OD-H column with hexane $/ 2$-propanol $=90 / 10$, flow $=1.0 \mathrm{ml} / \mathrm{min}$, wavelength $=254 \mathrm{~nm}$. Retention time $15.83 \mathrm{mins}$ (S-isomer), 33.17 mins (R-isomer). ${ }^{1} \mathrm{H}$ NMR $\left(\mathrm{CDCl}_{3}\right) \delta 7.90(\mathrm{~d}, J=2.8 \mathrm{~Hz}, 1 \mathrm{H}), 7.78(\mathrm{dd}, J=4.8,1.2$ $\mathrm{Hz}, 1 \mathrm{H}), 7.09-7.24(\mathrm{~m}, 5 \mathrm{H}), 6.83(\mathrm{dd}, J=8.4,4.8 \mathrm{~Hz}, 1 \mathrm{H}), 6.58(\mathrm{ddd}, J=8.4,2.8,1.2 \mathrm{~Hz}, 1 \mathrm{H}), 4.39$ (s, b, $1 \mathrm{H}), 4.30-4.38(\mathrm{~m}, 1 \mathrm{H}), 1.40(\mathrm{~d}, J=6.8 \mathrm{~Hz}, 3 \mathrm{H}) ;{ }^{13} \mathrm{C} \mathrm{NMR}\left(\mathrm{CDCl}_{3}\right) 144.15,143.18,138.16$, 136.33, 128.58, 126.93, 125.57, 123.41, 118.71, 53.03, 24.75. Anal. Calcd. For $\mathrm{C}_{13} \mathrm{H}_{14} \mathrm{~N}_{2}: \mathrm{C}, 78.75 ; \mathrm{H}$, 7.12; N, 14.13. Found: C, 78.44; H, 7.35; N, 13.84 .<smiles>CC(Nc1cccnc1)c1ccccc1</smiles>

$\mathrm{N}$-Octyl-4-aminopyridine. (Table 1, entry 22). The general procedure A conducted with 4chloropyridine hydrochloride $(0.150 \mathrm{~g}, 1.00 \mathrm{mmol})$, octylamine $(0.155 \mathrm{~g}, 1.20 \mathrm{mmol}), \mathrm{Pd}(\mathrm{OAc})_{2}$ and CyPF- $t$-Bu $\left(10.0 \mu \mathrm{L}\right.$ from stock solution A, $\left.1.0 \times 10^{-4} \mathrm{mmol}\right)$ gave $171.5 \mathrm{mg}(83 \%)$ of $N$-octyl-4aminopyridine as a yellow solid. ${ }^{1} \mathrm{H}$ NMR $\left(\mathrm{CDCl}_{3}\right) \delta 8.11(\mathrm{~d}, J=4.8 \mathrm{~Hz}, 2 \mathrm{H}), 6.37(\mathrm{~d}, J=4.8 \mathrm{~Hz}, 2$ H), 4.44 (s, b, $1 \mathrm{H}$ ), 3.08 (q, $J=7.2 \mathrm{~Hz}, 2 \mathrm{H}$ ), 1.55 (quint, $J=7.2 \mathrm{~Hz}, 2 \mathrm{H}$ ), 1.19-1.39 (m, $10 \mathrm{H}$ ), 0.84 $(\mathrm{t}, J=7.2 \mathrm{~Hz}, 3 \mathrm{H}) ;{ }^{13} \mathrm{C} \mathrm{NMR}\left(\mathrm{CDCl}_{3}\right) \delta 153.50,149.48,107.18,42.38,31.59,29.12,29.02,28.84$, 26.85, 22.44, 13.89; Anal. Calcd. For $\mathrm{C}_{13} \mathrm{H}_{22} \mathrm{~N}_{2}$ : C, 75.68; H, 10.75; N, 13.58. Found: C, 75.45; H, 10.80 ; N, 13.62 . 
NHoctyl

N-Octyl-4-aminopyridine. (Table 1, entry 23). The general procedure A conducted with 4bromopyridine hydrochloride $(0.195 \mathrm{~g}, 1.00 \mathrm{mmol})$, octylamine $(0.155 \mathrm{~g}, 1.20 \mathrm{mmol}), \mathrm{Pd}(\mathrm{OAc})_{2}$ and CyPF-t-Bu $\left(5.0 \mu \mathrm{L}\right.$ from stock solution A, $\left.5.0 \times 10^{-5} \mathrm{mmol}\right)$ gave $192.3 \mathrm{mg}(93 \%)$ of $N$-octyl-4aminopyridine as a white solid. $\left({ }^{1} \mathrm{H}\right.$ and ${ }^{13} \mathrm{C}$ NMR spectra are the same as those for the product in Table 1, entry 22.)

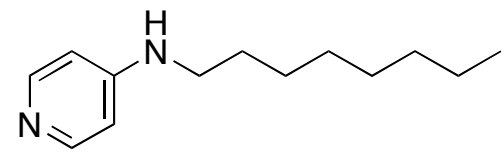

N-Octyl-4-aminopyridine. (Table 1, entry 24). The general procedure A conducted with 4 iodopyridine $(0.205 \mathrm{~g}, 1.00 \mathrm{mmol})$, octylamine $(0.155 \mathrm{~g}, 1.20 \mathrm{mmol}), \mathrm{Pd}(\mathrm{OAc})_{2}$ and CyPF- $t$-Bu $(50.0$ $\mu \mathrm{L}$ from stock solution $\left.\mathbf{A}, 5.0 \times 10^{-4} \mathrm{mmol}\right)$ and sodium tert-butoxide $(0.135 \mathrm{~g}, 1.40 \mathrm{mmol})$ gave 164.6 mg $(80 \%)$ of $N$-octyl-4-aminopyridine as a yellow solid. $\left({ }^{1} \mathrm{H}\right.$ and ${ }^{13} \mathrm{C}$ NMR spectra are the same as those for the product in Table 1, entry 22.)

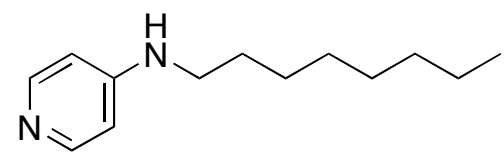

4-Benzamidopyridine. ${ }^{4}$ (Table 1, entry 25). The same procedure A conducted with 4-chloropyridine hydrochloride $(0.150 \mathrm{~g}, 1.00 \mathrm{mmol})$, benzamide $(0.145 \mathrm{~g}, 1.20 \mathrm{mmol}), \mathrm{Pd}(\mathrm{OAc})_{2}\left(2.2 \mathrm{mg}, 1.0 \times 10^{-2}\right.$ $\mathrm{mmol})$ and CyPF- $t$-Bu $\left(5.5 \mathrm{mg}, 1.0 \times 10^{-2} \mathrm{mmol}\right)$ gave $148.5 \mathrm{mg}(75 \%)$ of 4-benzamidopyridine as a white solid. ${ }^{1} \mathrm{H}$ NMR $\left(\mathrm{CD}_{3} \mathrm{COCD}_{3}\right) \delta 9.82(\mathrm{~s}, \mathrm{~b}, 1 \mathrm{H}), 8.48(\mathrm{~d}, J=6.4 \mathrm{~Hz}, 2 \mathrm{H}), 8.00(\mathrm{dt}, J=7.2,2.0$ $\mathrm{Hz}, 2 \mathrm{H}), 7.81(\mathrm{dd}, J=6.8,2.0 \mathrm{~Hz}, 2 \mathrm{H}), 7.60(\mathrm{tt}, J=7.2,1.4 \mathrm{~Hz}, 1 \mathrm{H}), 7.52(\mathrm{tt}, J=7.6,1.2 \mathrm{~Hz}, 2 \mathrm{H})$; ${ }^{13} \mathrm{C}$ NMR $\left(\mathrm{CD}_{3} \mathrm{COCD}_{3}\right) \delta 166.95,151.20,146.76,135.36,132.74,129.24,128.34,114.54$.<smiles>O=C(Nc1ccncc1)c1ccccc1</smiles>

N-Octylaminopyrazine. (Table 1, entry 26). The general procedure A conducted with chloropyrazine $(0.115 \mathrm{~g}, 1.00 \mathrm{mmol})$, octylamine $(0.155 \mathrm{~g}, 1.20 \mathrm{mmol}), \mathrm{Pd}(\mathrm{OAc})_{2}$ and CyPF- $t$-Bu $(5.0 \mu \mathrm{L}$ from stock solution A, $\left.5.0 \times 10^{-5} \mathrm{mmol}\right)$ gave $170.0 \mathrm{mg}(82 \%)$ of $N$-octylaminopyrazine as a pale yellow solid. ${ }^{1} \mathrm{H}$ NMR $\left(\mathrm{CDCl}_{3}\right) \delta 7.89(\mathrm{dd}, J=2.8,1.6 \mathrm{~Hz}, 1 \mathrm{H}), 7.80(\mathrm{~d}, J=1.6 \mathrm{~Hz}, 1 \mathrm{H}), 7.69(\mathrm{~d}, J=2.8 \mathrm{~Hz}, 1 \mathrm{H})$, $4.94(\mathrm{~s}, \mathrm{~b}, 1 \mathrm{H}), 3.24(\mathrm{q}, J=6.7 \mathrm{~Hz}, 2 \mathrm{H}), 1.54$ (quint, $J=7.3 \mathrm{~Hz}, 2 \mathrm{H}), 1.20-1.42(\mathrm{~m}, 10 \mathrm{H}), 0.80$ (t, $J$ $=6.8 \mathrm{~Hz}, 3 \mathrm{H}) ;{ }^{13} \mathrm{C} \mathrm{NMR}\left(\mathrm{CDCl}_{3}\right) \delta 154.70,141.83,132.16,131.72,41.40,31.65,29.28,29.17,29.09$, 26.87, 22.50, 13.96; Anal. Calcd. For $\mathrm{C}_{12} \mathrm{H}_{21} \mathrm{~N}_{3}$ : C, 69.52; H, 10.21; N, 20.27. Found: C, 69.58; H, $10.26 ; \mathrm{N}, 20.45$.<smiles>Oc1cnccn1</smiles>

N-Octylaminopyrazine. (Table 1, entry 27). The general procedure A conducted with iodopyrazine $(0.206 \mathrm{~g}, 1.00 \mathrm{mmol})$, octylamine $(0.155 \mathrm{~g}, 1.20 \mathrm{mmol}), \mathrm{Pd}(\mathrm{OAc})_{2}$ and $\mathrm{CyPF}-t-\mathrm{Bu}(50.0 \mu \mathrm{L}$ from stock 
solution A, $\left.5.0 \times 10^{-45} \mathrm{mmol}\right)$ gave $172.1 \mathrm{mg}(83 \%)$ of $N$-octylaminopyrazine as a pale yellow solid. $\left({ }^{1} \mathrm{H}\right.$ and ${ }^{13} \mathrm{C}$ NMR spectra are the same as those for the product in Table 1, entry 26.)<smiles>Oc1cnccn1</smiles>

5-(Cyclohexylamino)pyrimidine. ${ }^{5}$ (Table 1, entry 28). The general procedure A conducted with 5bromopyrimidine $(0.158 \mathrm{~g}, 1.00 \mathrm{mmol})$, cyclohexylamine $(0.119 \mathrm{~g}, 1.20 \mathrm{mmol}), \mathrm{Pd}(\mathrm{OAc})_{2}(2.2 \mathrm{mg}, 1.0$ $\mathrm{mmol})$ and CyPF- $t$-Bu (5.5 mg, $1.0 \mathrm{mmol})$ gave $141.2 \mathrm{mg}(80 \%)$ of 5-(cyclohexylamino)pyrimidine as a pale yellow plate. ${ }^{1} \mathrm{H}$ NMR $\left(\mathrm{CDCl}_{3}\right) \delta 8.53(\mathrm{~s}, 1 \mathrm{H}), 8.07(\mathrm{~s}, 2 \mathrm{H}), 3.63(\mathrm{~s}, \mathrm{~b}, 1 \mathrm{H}), 3.23-3.30(\mathrm{~m}, 1$ $\mathrm{H}), 2.03-2.06(\mathrm{~m}, 2 \mathrm{H}), 1.65-1.80(\mathrm{~m}, 3 \mathrm{H}), 1.15-1.43(\mathrm{~m}, 5 \mathrm{H}) ;{ }^{13} \mathrm{C} \mathrm{NMR}\left(\mathrm{CDCl}_{3}\right) \delta$ 148.01, 140.96, $105.28,51.12,32.94,25.58,24.66$.<smiles>c1ncc(NC2CCCCC2)cn1</smiles>

$\mathrm{N}$-Cyclohexyl-2-aminoquinoline. (Table 1, entry 29). The general procedure A conducted with 2chloroquinoline $(0.163 \mathrm{~g}, 1.00 \mathrm{mmol})$, cyclohexylamine $(0.119 \mathrm{~g}, 1.20 \mathrm{mmol}), \mathrm{Pd}(\mathrm{OAc})_{2}$ and CyPF- $t$ $\mathrm{Bu}\left(10.0 \mu \mathrm{L}\right.$ from stock solution $\left.\mathbf{A}, 1.0 \times 10^{-4} \mathrm{mmol}\right)$ gave $138.4 \mathrm{mg}(79 \%)$ of $N$-cyclohexyl-2aminoquinoline as a white solid. ${ }^{1} \mathrm{H} \mathrm{NMR}\left(\mathrm{CDCl}_{3}\right) \delta 7.67(\mathrm{~d}, J=9.2 \mathrm{~Hz}, 1 \mathrm{H}), 7.56(\mathrm{dd}, J=8.4,0.4$ $\mathrm{Hz}, 1 \mathrm{H}), 7.45(\mathrm{dd}, J=8.4,1.2 \mathrm{~Hz}, 1 \mathrm{H}), 7.40(\mathrm{~d}, J=8.8 \mathrm{~Hz}, 1 \mathrm{H}), 7.07$ (ddd, $J=8.0,7.0,1.2 \mathrm{~Hz}, 1$ $\mathrm{H}), 6.50(\mathrm{~d}, J=8.8 \mathrm{~Hz}, 1 \mathrm{H}), 4.66(\mathrm{~d}, J=8.0 \mathrm{~Hz}, 1 \mathrm{H}), 3.71-3.78(\mathrm{~m}, 1 \mathrm{H}), 1.97-2.01(\mathrm{~m}, 2 \mathrm{H}), 1.66$ $(\mathrm{dt}, J=13.6,4.9 \mathrm{~Hz}, 2 \mathrm{H}), 1.55(\mathrm{dt}, J=13.2,4.9 \mathrm{~Hz}, 1 \mathrm{H}), 1.27-1.38(\mathrm{~m}, 2 \mathrm{H}), 1.06-1.17(\mathrm{~m}, 3 \mathrm{H}) ;{ }^{13} \mathrm{C}$ NMR $\left(\mathrm{CDCl}_{3}\right) \delta 156.26,148.15,137.14,129.34,127.28,125.85,123.17,121.60,111.02,49.70,33.38$, 25.66, 24.82; Anal. Calcd. For $\mathrm{C}_{15} \mathrm{H}_{18} \mathrm{~N}_{2}$ : C, 79.61; H, 8.02; N, 12.38. Found: C, 79.49; H, 8.03; N, 12.23 .

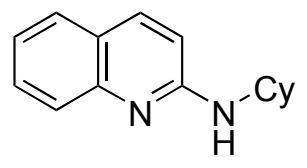

$\boldsymbol{N}$-Octyl-1-aminoisoquinoline. (Table 1, entry 30). The general procedure A conducted with 1chloroisoquinoline $(0.164 \mathrm{~g}, 1.00 \mathrm{mmol})$, octylamine $(0.155 \mathrm{~g}, 1.20 \mathrm{mmol}), \mathrm{Pd}(\mathrm{OAc})_{2}$ and $\mathrm{CyPF}-t-\mathrm{Bu}$ $\left(5.0 \mu \mathrm{L}\right.$ from stock solution $\left.\mathbf{A}, 5.0 \times 10^{-5} \mathrm{mmol}\right)$ gave $233.8 \mathrm{mg}(91 \%)$ of $N$-octyl-1-aminoisoquinoline as a pale yellow liquid. ${ }^{1} \mathrm{H} \mathrm{NMR}\left(\mathrm{CDCl}_{3}\right) \delta 7.98(\mathrm{~d}, J=6.0 \mathrm{~Hz}, 1 \mathrm{H}), 7.72(\mathrm{~d}, J=7.6 \mathrm{~Hz}, 1 \mathrm{H}), 7.65(\mathrm{~d}$, $J=8.4 \mathrm{~Hz}, 1 \mathrm{H}), 7.56(\mathrm{dd}, J=8.0,6.8 \mathrm{~Hz}, 1 \mathrm{H}), 7.43(\mathrm{dd}, J=8.2,6.8 \mathrm{~Hz}, 1 \mathrm{H}), 6.89(\mathrm{~d}, J=5.6 \mathrm{~Hz}, 1$ H), $5.16(\mathrm{~s}, \mathrm{~b}, 1 \mathrm{H}), 3.58(\mathrm{q}, J=7.6 \mathrm{~Hz}, 2 \mathrm{H}$ ), 1.72 (quint, $J=7.6 \mathrm{~Hz}, 2 \mathrm{H}$ ), 1.45 (quint, $J=7.6 \mathrm{~Hz}, 2$ $\mathrm{H}), 1.23-1.37(\mathrm{~m}, 8 \mathrm{H}), 0.86(\mathrm{t}, J=6.8 \mathrm{~Hz}, 3 \mathrm{H}) ;{ }^{13} \mathrm{C} \mathrm{NMR}\left(\mathrm{CDCl}_{3}\right) \delta 155.07,141.22,136.82,129.32$, 126.87, 125.49, 121.25, 117.96, 110.32, 41.77, 31.66, 29.43, 29.27, 29.11, 27.09, 22.49, 13.95; Anal. Calcd. For $\mathrm{C}_{17} \mathrm{H}_{24} \mathrm{~N}_{2}$ : C, 79.64; H, 9.44; N, 10.93. Found: C, 79.56; H, 9.57; N, 10.90 .<smiles>COc1nccc2ccccc12</smiles>

N-Octyl-4-amino-isoquinoline. (Table 1, entry 31). The general procedure A conducted with 4bromo-isoquinoline $(0.208 \mathrm{~g}, 1.00 \mathrm{mmol})$, cyclohexylamine $(0.155 \mathrm{~g}, 1.20 \mathrm{mmol}), \mathrm{Pd}(\mathrm{OAc})_{2}$ and 
CyPF- $t$-Bu (5.0 $\mu \mathrm{L}$ from stock solution $\left.\mathbf{A}, 5.0 \times 10^{-5} \mathrm{mmol}\right)$ gave $237.0 \mathrm{mg}(93 \%)$ of $N$-Octyl-4amino-isoquinoline as a white solid. ${ }^{1} \mathrm{H}$ NMR $\left(\mathrm{CDCl}_{3}\right) \delta 8.65(\mathrm{~s}, 1 \mathrm{H}), 7.86(\mathrm{~d}, J=8.0 \mathrm{~Hz}, 1 \mathrm{H}), 7.84$ (s, $1 \mathrm{H}), 7.75(\mathrm{~d}, J=8.5 \mathrm{~Hz}, 1 \mathrm{H}), 7.60(\mathrm{t}, J=8.0 \mathrm{~Hz}, 1 \mathrm{H}), 7.53(\mathrm{t}, J=7.5 \mathrm{~Hz}, 1 \mathrm{H}), 4.19(\mathrm{~s}, 1 \mathrm{H}), 3.27$ (q, $J=7.0 \mathrm{~Hz}, 2 \mathrm{H}$ ), 1.74 (quint, $J=8.0 \mathrm{~Hz}, 2 \mathrm{H}$ ), 1.45 (quint, $J=7.0 \mathrm{~Hz}, 2 \mathrm{H}$ ), $1.27-1.36(\mathrm{~m}, 8 \mathrm{H}$ ), $0.87(\mathrm{t}, J=7.0 \mathrm{~Hz}, 3 \mathrm{H}) ;{ }^{13} \mathrm{C} \mathrm{NMR}\left(\mathrm{CDCl}_{3}\right) \delta 141.70,137.84,128.73,128.38,127.93,126.76,125.64$, 123.26, 119.04, 43.87, 31.75, 29.35, 29.32, 29.19, 27.18, 22.59, 14.05. Anal. Calcd. For $\mathrm{C}_{17} \mathrm{H}_{24} \mathrm{~N}_{2}$ : C, 79.64; H, 9.44; N, 10.93. Found: C, 79.52; H, 9.82; N, 10.90.

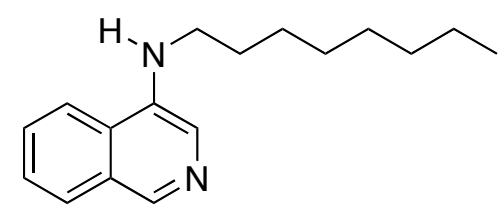

$N$-(Phenyl)cyclohexylamime. ${ }^{6}$ (Table 3, entry 1). The general procedure A conducted with phenylchloride $(0.113 \mathrm{~g}, 1.00 \mathrm{mmol})$, cyclohexylamine $(0.119 \mathrm{~g}, 1.20 \mathrm{mmol}), \mathrm{Pd}(\mathrm{OAc})_{2}$ and CyPF- $t$ $\mathrm{Bu}\left(5.0 \mu \mathrm{L}\right.$ from stock solution $\left.\mathbf{A}, 5.0 \times 10^{-5} \mathrm{mmol}\right)$ gave $174.7 \mathrm{mg}(99 \%)$ of $\mathrm{N}$ (phenyl)cyclohexylamine as a pale yellow liquid. ${ }^{1} \mathrm{H} \mathrm{NMR}\left(\mathrm{CDCl}_{3}\right) \delta 7.05(\mathrm{t}, J=7.6 \mathrm{~Hz}, 2 \mathrm{H}), 6.56$ $(\mathrm{td}, J=7.2,1.2 \mathrm{~Hz}, 1 \mathrm{H}), 6.48(\mathrm{dd}, J=7.6,0.8 \mathrm{~Hz}, 2 \mathrm{H}), 3.38(\mathrm{~s}, \mathrm{~b}, 1 \mathrm{H}), 3.11-3.18(\mathrm{~m}, 1 \mathrm{H}), 1.94-1.97$ $(\mathrm{m}, 2 \mathrm{H}), 1.66$ (dt, $J=13.2,4.0 \mathrm{~Hz}, 2 \mathrm{H}), 1.55$ (dt, $J=12.0,3.6 \mathrm{~Hz}, 1 \mathrm{H}), 1.22-1.32(\mathrm{~m}, 2 \mathrm{H}), 0.99$ $1.18(\mathrm{~m}, 3 \mathrm{H}) ;{ }^{13} \mathrm{C} \mathrm{NMR}\left(\mathrm{CDCl}_{3}\right) \delta 147.31,129.16,116.71,113.02,51.56,33.39,25.88,24.96$.<smiles>c1ccc2c(c1)N[Al]N2</smiles>

$\mathrm{N}$-cyclohexyl-aniline. ${ }^{6}$ (Table 3, entry 2). The general procedure A conducted with phenyl bromide $(0.157 \mathrm{~g}, 1.00 \mathrm{mmol})$, cyclohexylamine $(0.119 \mathrm{~g}, 1.20 \mathrm{mmol}), \mathrm{Pd}(\mathrm{OAc})_{2}$ and CyPF- $t$-Bu $(10.0 \mu \mathrm{L}$ from stock solution A, $\left.1.0 \times 10^{-4} \mathrm{mmol}\right)$ gave $168.9 \mathrm{mg}(96 \%)$ of $\mathrm{N}$-cyclohexyl-aniline as a white solid. $\left({ }^{1} \mathrm{H}\right.$ and ${ }^{13} \mathrm{C}$ NMR spectra are the same as those for the product in Table 3, entry 1.)

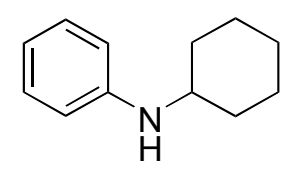

$\boldsymbol{N}$-(Phenyl)benzylamine. ${ }^{6}$ (Table 3, entry 3). The general procedure A conducted with phenyl chloride $(0.113 \mathrm{~g}, 1.00 \mathrm{mmol})$, benzylamine $(0.129 \mathrm{~g}, 1.20 \mathrm{mmol}), \mathrm{Pd}(\mathrm{OAc})_{2}$ and CyPF-t-Bu (5.0 $\mu \mathrm{L}$ from stock solution A, $5.0 \times 10^{-5} \mathrm{mmol}$ ) gave $183.0 \mathrm{mg}(99 \%)$ of $N$-(Phenyl)benzylamine as a pale yellow liquid. ${ }^{1} \mathrm{H}$ NMR $\left(\mathrm{CDCl}_{3}\right) \delta 7.49(\mathrm{~d}, J=6.0 \mathrm{~Hz}, 2 \mathrm{H}), 7.46(\mathrm{~d}, J=7.2 \mathrm{~Hz}, 2 \mathrm{H}), 7.38-7.41(\mathrm{~m}, 1$ $\mathrm{H}), 7.30(\mathrm{t}, J=7.2 \mathrm{~Hz}, 2 \mathrm{H}), 6.86(\mathrm{td}, J=7.2,1.2 \mathrm{~Hz}, 1 \mathrm{H}), 6.74(\mathrm{dd}, J=7.6,1.2 \mathrm{~Hz}, 2 \mathrm{H}), 4.42(\mathrm{~s}, 2$ $\mathrm{H}), 4.09(\mathrm{~s}, \mathrm{~b}, 1 \mathrm{H}) ;{ }^{13} \mathrm{C}$ NMR $\left(\mathrm{CDCl}_{3}\right) \delta 148.04,139.36,129.15,128.51,127.37,127.09,117.41$, $112.73,48.13$.<smiles>BrCNc1ccccc1</smiles>

$N$-Benzylaniline. ${ }^{6}$ (Table 3, entry 4). The general procedure A conducted with phenyl bromide $(0.157$ $\mathrm{g}, 1.00 \mathrm{mmol})$, benzylamine $(0.129 \mathrm{~g}, 1.20 \mathrm{mmol}), \mathrm{Pd}(\mathrm{OAc})_{2}$ and $\mathrm{CyPF}-\mathrm{t}-\mathrm{Bu}(100.0 \mu \mathrm{L}$ from stock 
solution B, $\left.1.0 \times 10^{-5} \mathrm{mmol}\right)$ gave $178.1 \mathrm{mg}(97 \%)$ of $N$-Benzylaniline as a white solid. $\left({ }^{1} \mathrm{H}\right.$ and ${ }^{13} \mathrm{C}$ NMR spectra are the same as those for the product in Table 3, entry 3.)<smiles>c1ccc(CNc2ccccc2)cc1</smiles>

$N$-(o-Tolyl)octylamine. (Table 3, entry 5). The general procedure A conducted with 2-chlorotoluene $(0.127 \mathrm{~g}, 1.00 \mathrm{mmol})$, octylamine $(0.155 \mathrm{~g}, 1.20 \mathrm{mmol}), \mathrm{Pd}(\mathrm{OAc})_{2}$ and $\mathrm{CyPF}-t-\mathrm{Bu}(10.0 \mu \mathrm{L}$ from stock solution A, $\left.1.0 \times 10^{-4} \mathrm{mmol}\right)$ gave $214.1 \mathrm{mg}(98 \%)$ of $N$-(o-tolyl)octylamine as a yellow solid. ${ }^{1} \mathrm{H}$ $\operatorname{NMR}\left(\mathrm{CDCl}_{3}\right) \delta 7.20(\mathrm{t}, J=7.6 \mathrm{~Hz}, 1 \mathrm{H}), 7.12(\mathrm{~d}, J=7.2 \mathrm{~Hz}, 1 \mathrm{H}), 6.72(\mathrm{~d}, J=7.4 \mathrm{~Hz}, 1 \mathrm{H}), 6.69(\mathrm{~d}, J$ $=8.0 \mathrm{~Hz}, 1 \mathrm{H}), 3.50(\mathrm{~s}, \mathrm{~b}, 1 \mathrm{H}), 3.22(\mathrm{t}, J=7.2 \mathrm{~Hz}, 2 \mathrm{H}), 2.21(\mathrm{~s}, 3 \mathrm{H}), 1.74$ (quint, $J=7.3 \mathrm{~Hz}, 2 \mathrm{H})$, 1.38-1.52 (m, $10 \mathrm{H}), 0.99(\mathrm{t}, J=7.2 \mathrm{~Hz}, 3 \mathrm{H}) ;{ }^{13} \mathrm{C}$ NMR $\left(\mathrm{CDCl}_{3}\right) \delta 146.35,129.92,127.07,121.55$, 116.53, 109.52, 43.91, 31.82, 29.59, 29.42, 29.26, 27.23, 22.65, 17.38, 14.00; Anal. Calcd. For $\mathrm{C}_{15} \mathrm{H}_{25} \mathrm{~N}$ : C, 82.13; H, 11.94; N, 6.39. Found: C, 82.23; H, 11.67; N, 6.10.

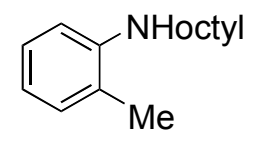

$\mathbf{N}$-(o-Tolyl)octylamine. (Table 3, entry 6). The general procedure A conducted with 2-bromotoluene $(0.171 \mathrm{~g}, 1.00 \mathrm{mmol})$, octylamine $(0.155 \mathrm{~g}, 1.20 \mathrm{mmol}), \mathrm{Pd}(\mathrm{OAc})_{2}$ and $\mathrm{CyPF}-\mathrm{t}-\mathrm{Bu}(5.0 \mu \mathrm{L}$ from stock solution A, $\left.5.0 \times 10^{-5} \mathrm{mmol}\right)$ gave $218.4 \mathrm{mg}(99 \%)$ of $N$ - $\left(o\right.$-tolyl)octylamine as a yellow solid. $\left({ }^{1} \mathrm{H}\right.$ and ${ }^{13} \mathrm{C}$ NMR spectra are the same as those for the product in Table 3 , entry 5 .)

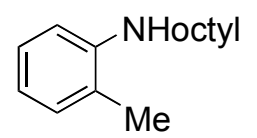

$N$-(o-Tolyl)octylamine. (Table 3, entry 7). The general procedure conducted with 2-iodotoluene $(0.218 \mathrm{~g}, 1.00 \mathrm{mmol})$, octylamine $(0.155 \mathrm{~g}, 1.20 \mathrm{mmol}), \mathrm{Pd}(\mathrm{OAc})_{2}$ and CyPF-t-Bu (50.0 $\mu \mathrm{L}$ from stock solution $\left.\mathbf{A}, 5.0 \times 10^{-4} \mathrm{mmol}\right)$ and sodium tert-butoxide $(0.135 \mathrm{~g}, 1.40 \mathrm{mmol})$ gave $203.7 \mathrm{mg}(93 \%)$ of $N$-(o-tolyl)octylamine as a yellow solid. $\left({ }^{1} \mathrm{H}\right.$ and ${ }^{13} \mathrm{C}$ NMR spectra are the same as those for the product in Table 3, entry 5.)

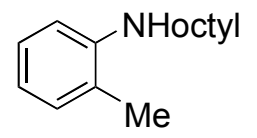

N-(Cyclohexylamino)-o-anisidine. (Table 3, entry 8). The general procedure A conducted with 2bromoanisole $(0.187 \mathrm{~g}, 1.00 \mathrm{mmol})$, cyclohexylamine $(0.119 \mathrm{~g}, 1.20 \mathrm{mmol}), \mathrm{Pd}(\mathrm{OAc})_{2}$ and CyPF-t-Bu $\left(50.0 \mu \mathrm{L}\right.$ from stock solution $\left.\mathbf{A}, 5.0 \times 10^{-4} \mathrm{mmol}\right)$ gave $192.0 \mathrm{mg}(94 \%)$ of $N$-(cyclohexylamino)- $O$ anisidine as a pale yellow liquid. ${ }^{1} \mathrm{H} \mathrm{NMR}\left(\mathrm{CDCl}_{3}\right) \delta 6.88(\mathrm{td}, J=8.0,1.6 \mathrm{~Hz}, 1 \mathrm{H}), 6.78(\mathrm{dd}, J=8.4$, $1.2 \mathrm{~Hz}, 1 \mathrm{H}), 6.23-6.67(\mathrm{~m}, 2 \mathrm{H}), 4.17$ (s, b, $1 \mathrm{H}), 3.86(\mathrm{~s}, 3 \mathrm{H}), 3.28$ (tt, $J=10.0,3.8 \mathrm{~Hz}, 1 \mathrm{H}), 2.10$ $(\mathrm{dd}, J=12.0,3.0 \mathrm{~Hz}, 2 \mathrm{H}), 1.80(\mathrm{dt}, J=12.8,3.6 \mathrm{~Hz}, 2 \mathrm{H}), 1.68(\mathrm{dt}, J=12.8,4.0 \mathrm{~Hz}, 1 \mathrm{H}), 1.35-1.46$ $(\mathrm{m}, 2 \mathrm{H}), 1.18-1.31(\mathrm{~m}, 3 \mathrm{H}) ;{ }^{13} \mathrm{C} \mathrm{NMR}\left(\mathrm{CDCl}_{3}\right) \delta 146.62,137.19,121.16,115.67,110.09,109.47$, 
55.28, 51.25, 33.37, 25.96, 25.03; Anal. Calcd. For $\mathrm{C}_{13} \mathrm{H}_{19} \mathrm{~N}_{2} \mathrm{O}$ : C, 76.06; H, 9.33; N, 6.82. Found: C, 76.04; H, 9.32; N, 6.71.<smiles>COc1ccccc1NC(Cl)Cl</smiles>

$\boldsymbol{N}$-p-Tolyl-iso-butylamine. ${ }^{7}$ (Table 3, entry 9). The general procedure A conducted with 4chlorotoluene $(0.127 \mathrm{~g}, 1.00 \mathrm{mmol})$, iso-butylamine $(87.8 \mathrm{mg}, 1.20 \mathrm{mmol}), \mathrm{Pd}(\mathrm{OAc})_{2}$ and CyPF-t-Bu $\left(50.0 \mu \mathrm{L}\right.$ from stock solution $\left.\mathbf{A}, 5.0 \times 10^{-4} \mathrm{mmol}\right)$ gave $135.5 \mathrm{mg}(83 \%)$ of $N$-p-tolyl-iso-butylamine as a pale yellow liquid. ${ }^{1} \mathrm{H}$ NMR $\left(\mathrm{CDCl}_{3}\right) \delta 6.99(\mathrm{~d}, J=8.0 \mathrm{~Hz}, 2 \mathrm{H}), 6.54(\mathrm{~d}, J=7.8 \mathrm{~Hz}, 2 \mathrm{H}), 3.47(\mathrm{~s}, \mathrm{~b}$, $1 \mathrm{H}), 2.92(\mathrm{~d}, J=6.8 \mathrm{~Hz}, 2 \mathrm{H}), 2.25(\mathrm{~s}, 3 \mathrm{H}), 1.78$ (nonet, $J=6.8 \mathrm{~Hz}, 1 \mathrm{H}), 0.99$ (d, $J=6.8 \mathrm{~Hz}, 6 \mathrm{H})$; ${ }^{13} \mathrm{C} \mathrm{NMR}\left(\mathrm{CDCl}_{3}\right) \delta 146.29,129.66,126.11,112.83,52.20,27.97,20.46,20.33$.<smiles>Cc1ccc(NCC(C)C)cc1</smiles>

$\boldsymbol{N}$-p-Tolyl-iso-butylamine. (Table 3 , entry 10). The general procedure A conducted with 4bromotoluene $(0.171 \mathrm{~g}, 1.00 \mathrm{mmol})$, iso-butylamine $(87.8 \mathrm{mg}, 1.20 \mathrm{mmol}), \mathrm{Pd}(\mathrm{OAc})_{2}$ and CyPF- $t$-Bu $\left(50.0 \mu \mathrm{L}\right.$ from stock solution $\left.\mathbf{A}, 5.0 \times 10^{-4} \mathrm{mmol}\right)$ gave $147.1 \mathrm{mg}(90 \%)$ of $N$-p-tolyl-iso-butylamine as a pale yellow liquid. $\left({ }^{1} \mathrm{H}\right.$ and ${ }^{13} \mathrm{C}$ NMR spectra are the same as those for the product in Table 3 , entry 9.)<smiles>Cc1ccc(NCC(C)C)cc1</smiles>

$N$-p-Tolyl-iso-butylamine. (Table 3, entry 11). The general procedure conducted with 4-iodotoluene $(0.218 \mathrm{~g}, 1.00 \mathrm{mmol})$, iso-butylamine $(87.8 \mathrm{mg}, 1.20 \mathrm{mmol}), \mathrm{Pd}(\mathrm{OAc})_{2}$ and $\mathrm{CyPF}-t$-Bu $(50.0 \mu \mathrm{L}$ from stock solution $\left.\mathbf{A}, 5.0 \times 10^{-4} \mathrm{mmol}\right)$ and sodium tert-butoxide $(0.135 \mathrm{~g}, 1.40 \mathrm{mmol})$ gave $122.5 \mathrm{mg}$ (75\%) of $N$-p-tolyl-iso-butylamine as a pale yellow liquid. $\left({ }^{1} \mathrm{H}\right.$ and ${ }^{13} \mathrm{C}$ NMR spectra are the same as those for the product in Table 3, entry 9.)<smiles>Cc1ccc(NCC(C)C)cc1</smiles>

$\boldsymbol{N}$-p-Tolyl-iso-butylamine. (Table 3, entry 12). The general procedure conducted with 4-iodotoluene (5.50 g, $25.0 \mathrm{mmol})$, iso-butylamine (2.20 g, $30.0 \mathrm{mmol}), \mathrm{Pd}(\mathrm{OAc})_{2}(1.1 \mathrm{mg}, 0.50 \mathrm{mmol})$ and CyPF- $t$ $\mathrm{Bu}(2.7 \mathrm{mg}, 0.50 \mathrm{mmol})$ and sodium tert-butoxide $(3.38 \mathrm{~g}, 35.0 \mathrm{mmol})$ gave $3.30 \mathrm{~g}(81 \%)$ of $N$-p-tolyliso-butylamine as a pale yellow liquid. $\left({ }^{1} \mathrm{H}\right.$ and ${ }^{13} \mathrm{C}$ NMR spectra are the same as those for the product in Table 3, entry 9.)<smiles>Cc1ccc(NCC(C)C)cc1</smiles> 
$N$-(p-Tolyl)-sec-butylamine. (Table 3, entry 13). The general procedure A conducted with 4bromotoluene $(0.171 \mathrm{~g}, 1.00 \mathrm{mmol})$, sec-butylamine $(87.8 \mathrm{mg}, 1.20 \mathrm{mmol}), \mathrm{Pd}(\mathrm{OAc})_{2}$ and CyPF- $t$-Bu $\left(50.0 \mu \mathrm{L}\right.$ from stock solution $\left.\mathbf{A}, 5.0 \times 10^{-4} \mathrm{mmol}\right)$ gave $142.2 \mathrm{mg}(87 \%)$ of $N$-(p-tolyl)-sec-butylamine as a colorless liquid. ${ }^{1} \mathrm{H}$ NMR $\left(\mathrm{CDCl}_{3}\right) \delta 7.01(\mathrm{~d}, J=8.4 \mathrm{~Hz}, 2 \mathrm{H}), 6.65(\mathrm{dd}, J=8.4 \mathrm{~Hz}, 2 \mathrm{H}), 3.40$ (sext, $J=6.0 \mathrm{~Hz}, 1 \mathrm{H}), 3.22(\mathrm{~s}, \mathrm{~b}, 1 \mathrm{H}), 2.27$ (s, $3 \mathrm{H}), 1.63$ (sept, $J=6.0 \mathrm{~Hz}, 1 \mathrm{H}), 1.49$ (sept, $J=7.0$ $\mathrm{Hz}, 1 \mathrm{H}), 1.19(\mathrm{~d}, J=6.4 \mathrm{~Hz}, 3 \mathrm{H}), 0.98(\mathrm{t}, J=7.2 \mathrm{~Hz}, 3 \mathrm{H}) ;{ }^{13} \mathrm{C} \mathrm{NMR}\left(\mathrm{CDCl}_{3}\right) \delta 145.40,129.70$, 125.91, 113.33, 50.04, 29.59, 20.30, 20.22, 10.32; Anal. Calcd. For $\mathrm{C}_{11} \mathrm{H}_{17} \mathrm{~N}: \mathrm{C}, 80.93 ; \mathrm{H}, 10.50 ; \mathrm{N}$, 8.58. Found: C, 80.71; H, 10.24; N, 8.66.

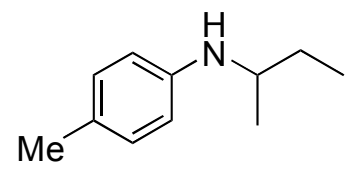

$N$-(p-Tolyl)-sec-butylamine. (Table 3, entry 14). The general procedure conducted with 4iodotoluene $(0.218 \mathrm{~g}, 1.00 \mathrm{mmol})$, sec-butylamine $(87.8 \mathrm{mg}, 1.20 \mathrm{mmol}), \mathrm{Pd}(\mathrm{OAc})_{2}(1.1 \mathrm{mg}, 0.50$ $\mathrm{mmol} \%)$ and CyPF- $t$-Bu $(2.7 \mathrm{mg}, 0.50 \mathrm{mmol} \%)$ and sodium tert-butoxide $(0.135 \mathrm{~g}, 1.40 \mathrm{mmol})$ gave $133.8 \mathrm{mg}(82 \%)$ of $\mathrm{N}$-(p-tolyl)-sec-butylamine as a colorless liquid. $\left({ }^{1} \mathrm{H}\right.$ and ${ }^{13} \mathrm{C}$ NMR spectra are the same as those for the product in Table 3 , entry 13.)<smiles>CCC(C)Nc1ccc(C)cc1</smiles>

N-(4-Methoxyphenyl)octylamine. (Table 3, entry 15). The general procedure A conducted with 4chloroanisole $(0.143 \mathrm{~g}, 1.00 \mathrm{mmol})$, octylamine $(0.155 \mathrm{~g}, 1.20 \mathrm{mmol}), \mathrm{Pd}(\mathrm{OAc})_{2}$ and CyPF- $t$-Bu $(100.0$ $\mu \mathrm{L}$ from stock solution $\left.\mathbf{A}, 1.0 \times 10^{-3} \mathrm{mmol}\right)$ gave $217.2 \mathrm{mg}(92 \%)$ of $N$-(4-methoxyphenyl)octylamine as a pale yellow liquid. ${ }^{1} \mathrm{H} \mathrm{NMR}\left(\mathrm{CDCl}_{3}\right) \delta 6.68(\mathrm{~d}, J=6.4 \mathrm{~Hz}, 2 \mathrm{H}), 6.47(\mathrm{~d}, J=6.8 \mathrm{~Hz}, 2 \mathrm{H}), 3.63(\mathrm{~s}$, $3 \mathrm{H}), 3.17(\mathrm{~s}, \mathrm{~b}, 1 \mathrm{H}), 2.94(\mathrm{t}, J=7.0 \mathrm{~Hz}, 2 \mathrm{H}), 1.49$ (quint, $J=7.0 \mathrm{~Hz}, 2 \mathrm{H}), 1.20-1.28(\mathrm{~m}, 10 \mathrm{H}), 0.80$ $(\mathrm{t}, J=6.8 \mathrm{~Hz}, 3 \mathrm{H}) ;{ }^{13} \mathrm{C} \mathrm{NMR}\left(\mathrm{CDCl}_{3}\right) \delta 151.77,142.76,114.71,113.83,55.60,44.88,31.75,29.58$, 29.37, 29.20, 27.13, 22.58, 14.02. Anal. Calcd. For $\mathrm{C}_{15} \mathrm{H}_{25} \mathrm{NO}$ : C, 76.55; H, 10.71; N, 5.95. Found: C, $76.46 ; \mathrm{H}, 10.41 ; \mathrm{N}, 6.04$.<smiles>COc1ccc(NOc2ccccc2)cc1</smiles>

N-(4-Methoxyphenyl)octylamine. (Table 3, entry 16). The general procedure conducted with 4iodoanisole $(0.234 \mathrm{~g}, 1.00 \mathrm{mmol})$, octylamine $(0.155 \mathrm{~g}, 1.20 \mathrm{mmol}), \mathrm{Pd}(\mathrm{OAc})_{2}(2.2 \mathrm{mg}, 1.0 \mathrm{mmol} \%)$ and $\mathrm{CyPF}-\mathrm{t}$-Bu $(5.5 \mathrm{mg}, 1.0 \mathrm{mmol} \%)$ and sodium tert-butoxide $(0.135 \mathrm{~g}, 1.40 \mathrm{mmol})$ gave $158.3 \mathrm{mg}$ (67\%) of $N$-(4-methoxyphenyl)octylamine as a pale yellow liquid. $\left({ }^{1} \mathrm{H}\right.$ and ${ }^{13} \mathrm{C} \mathrm{NMR}$ spectra are the same as those for the product in Table 3, entry 15.)<smiles>COc1ccc(NOc2ccccc2)cc1</smiles>

N-(4-Cyanophenyl)-iso-butylamine. (Table 3, entry 17). The general procedure A conducted with 4cyano-1-chlorobenzene $(0.138 \mathrm{~g}, 1.00 \mathrm{mmol})$, iso-butylamine $(87.8 \mathrm{mg}, 1.20 \mathrm{mmol}), \mathrm{Pd}(\mathrm{OAc})_{2}$ and CyPF- $t$-Bu $\left(5.0 \mu \mathrm{L}\right.$ from stock solution $\left.\mathbf{A}, 5.0 \times 10^{-5} \mathrm{mmol}\right)$ gave $156.0 \mathrm{mg}(90 \%)$ of $N$-(4cyanoxyphenyl)-iso-butylamine as a pale yellow liquid. ${ }^{1} \mathrm{H} \mathrm{NMR}\left(\mathrm{CDCl}_{3}\right) \delta 7.27(\mathrm{~d}, J=8.8 \mathrm{~Hz}, 2 \mathrm{H})$, $7.46(\mathrm{~d}, J=8.8 \mathrm{~Hz}, 2 \mathrm{H}), 4.32(\mathrm{~s}, \mathrm{~b}, 1 \mathrm{H}), 2.87$ (d, $J=6.8 \mathrm{~Hz}, 2 \mathrm{H}), 1.80$ (nonet, $J=6.8 \mathrm{~Hz}, 1 \mathrm{H}$ ), 0.89 
$(\mathrm{d}, J=6.8 \mathrm{~Hz}, 6 \mathrm{H}) ;{ }^{13} \mathrm{C} \mathrm{NMR}\left(\mathrm{CDCl}_{3}\right) \delta 151.74,133.43,120.38,112.01,98.00,50.86,27.90$, 20.12; Anal. Calcd. For $\mathrm{C}_{11} \mathrm{H}_{14} \mathrm{~N}_{2}$ : C, 75.82; H, 8.10; N, 16.08. Found: C, 75.96; H, 8.03; N, 16.01.

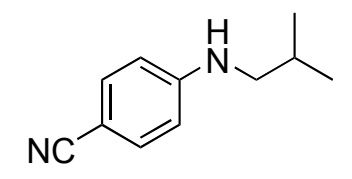

N-(4-Cyanophenyl)-cyclohexylamine. (Table 3, entry 18). The general procedure A conducted with 4-cyano-1-bromobenzene (0.182 g, $1.00 \mathrm{mmol})$, cyclohexylamine $(0.119 \mathrm{~g}, 1.20 \mathrm{mmol}), \mathrm{Pd}(\mathrm{OAc})_{2}$ and CyPF- $t$-Bu $\left(5.0 \mu \mathrm{L}\right.$ from stock solution $\left.\mathbf{A}, 5.0 \times 10^{-5} \mathrm{mmol}\right)$ gave $184.2 \mathrm{mg}(92 \%)$ of $N$-(4Cyanophenyl)-cyclohexylamine as a pale yellow liquid. ${ }^{1} \mathrm{H} \mathrm{NMR}\left(\mathrm{CDCl}_{3}\right) \delta 7.34(\mathrm{~d}, J=8.4 \mathrm{~Hz}, 2 \mathrm{H})$, $6.49(\mathrm{~d}, J=8.4 \mathrm{~Hz}, 2 \mathrm{H}), 4.21(\mathrm{~d}, J=7.6 \mathrm{~Hz}, 1 \mathrm{H}), 3.21-3.29(\mathrm{~m}, 1 \mathrm{H}), 1.97-2.01(\mathrm{~m}, 2 \mathrm{H}), 1.74(\mathrm{dt}, J$ $=13.2,4.0 \mathrm{~Hz}, 2 \mathrm{H}), 1.63(\mathrm{dt}, J=12.8,4.0 \mathrm{~Hz}, 1 \mathrm{H}), 1.29-1.40(\mathrm{~m}, 2 \mathrm{H}), 1.11-1.26(\mathrm{~m}, 3 \mathrm{H}) ;{ }^{13} \mathrm{C}$ NMR $\left(\mathrm{CDCl}_{3}\right) \delta 150.42,133.58,120.66,112.19,97.47,51.08,32.80,25.54,24.70$. Anal. Calcd. For $\mathrm{C}_{13} \mathrm{H}_{16} \mathrm{~N}_{2}$ : C, 77.96; H, 8.05; N, 13.99. Found: C, 77.85; H, 8.08; N, 13.78 .

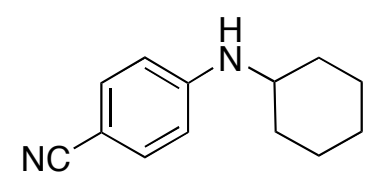

$N$-(Benzylamino)-m-anisidine. (Table 3, entry 19). The general procedure A conducted with 3chloroanisole $(0.143 \mathrm{~g}, 1.00 \mathrm{mmol})$, benzylamine $(0.129 \mathrm{~g}, 1.20 \mathrm{mmol}), \mathrm{Pd}(\mathrm{OAc})_{2}$ and CyPF-t-Bu $(5.0$ $\mu \mathrm{L}$ from stock solution $\left.\mathbf{A}, 5.0 \times 10^{-5} \mathrm{mmol}\right)$ gave $208.0 \mathrm{mg}(98 \%)$ of $N$-(benzylamino)- $m$-anisidine as a pale yellow liquid. ${ }^{1} \mathrm{H} \mathrm{NMR}\left(\mathrm{CDCl}_{3}\right) \delta 7.45(\mathrm{dd}, J=8.4,2.0 \mathrm{~Hz}, 2 \mathrm{H}), 7.42(\mathrm{t}, J=7.6 \mathrm{~Hz}, 2 \mathrm{H}), 7.36$ $(\mathrm{tt}, J=6.4,2.0 \mathrm{~Hz}, 1 \mathrm{H}), 7.17(\mathrm{t}, J=8.0 \mathrm{~Hz}, 1 \mathrm{H}), 6.39(\mathrm{dd}, J=8.0,1.6 \mathrm{~Hz}, 1 \mathrm{H}), 6.34(\mathrm{dd}, J=8.0,1.6$ $\mathrm{Hz}, 1 \mathrm{H}), 6.28(\mathrm{t}, J=1.6 \mathrm{~Hz}, 1 \mathrm{H}), 4.37(\mathrm{~s}, 2 \mathrm{H}), 4.13(\mathrm{~s}, \mathrm{~b}, 1 \mathrm{H}), 3.81(\mathrm{~s}, 3 \mathrm{H}) ;{ }^{13} \mathrm{C} \mathrm{NMR}\left(\mathrm{CDCl}_{3}\right) \delta$ $160.65,149.42$, 139.23, 129.84, 128.46, 127.33, 127.05, 105.80, 102.45, 98.70, 54.85, 48.06. Anal. Calcd. For $\mathrm{C}_{14} \mathrm{H}_{15} \mathrm{NO}$ : C, 78.84; H, 7.09; N, 6.57. Found: C, 78.83; H, 7.13; N, 6.54.

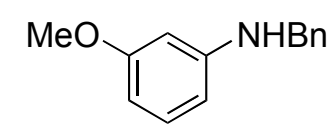

$\boldsymbol{N}$-(Benzylamino)- $\boldsymbol{m}$-anisidine. (Table 3, entry 20). The general procedure conducted with 3iodoanisole $(0.234 \mathrm{~g}, 1.00 \mathrm{mmol})$, benzylamine $(0.129 \mathrm{~g}, 1.20 \mathrm{mmol}), \mathrm{Pd}(\mathrm{OAc})_{2}$ and CyPF-t-Bu $(5.0$ $\mu \mathrm{L}$ from stock solution $\left.\mathbf{A}, 5.0 \times 10^{-5} \mathrm{mmol}\right)$ and sodium tert-butoxide $(0.135 \mathrm{~g}, 1.40 \mathrm{mmol})$ gave 211.6 $\mathrm{mg}(99 \%)$ of $N$-(benzylamino)- $m$-anisidine as a pale yellow liquid. $\left({ }^{1} \mathrm{H}\right.$ and ${ }^{13} \mathrm{C}$ NMR spectra are the same as those for the product in Table 3, entry 19.)

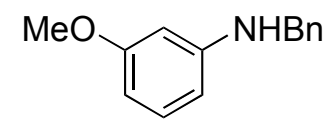

$N$-(Cyclohexylamino)- $\boldsymbol{m}$-anisidine. ${ }^{8}$ (Table 3, entry 21). The general procedure A conducted with 3bromoanisole $(0.143 \mathrm{~g}, 1.00 \mathrm{mmol})$, cyclohexylamine $(0.119 \mathrm{~g}, 1.20 \mathrm{mmol}), \mathrm{Pd}(\mathrm{OAc})_{2}$ and CyPF- $t$-Bu $\left(10.0 \mu \mathrm{L}\right.$ from stock solution $\left.\mathbf{A}, 1.0 \times 10^{-4} \mathrm{mmol}\right)$ gave $207.2 \mathrm{mg}(99 \%)$ of $N$-(cyclohexylamino)- $m$ anisidine as a pale yellow liquid. ${ }^{1} \mathrm{H}$ NMR $\left(\mathrm{CDCl}_{3}\right) \delta 7.08(\mathrm{t}, J=8.4 \mathrm{~Hz}, 1 \mathrm{H}), 6.26(\mathrm{dd}, J=8.4,2.0$ $\mathrm{Hz}, 1 \mathrm{H}), 6.23(\mathrm{dd}, J=8.0,2.0 \mathrm{~Hz}, 1 \mathrm{H}), 6.18(7, J=2.0 \mathrm{~Hz}, 1 \mathrm{H}), 3.78(\mathrm{~s}, 3 \mathrm{H}), 3.52$ (s, b, $1 \mathrm{H}), 3.28$ $(\mathrm{tt}, J=10.0,3.8 \mathrm{~Hz}, 1 \mathrm{H}), 2.08(\mathrm{dd}, J=12.6,3.0 \mathrm{~Hz}, 2 \mathrm{H}), 1.78(\mathrm{dt}, J=13.2,3.6 \mathrm{~Hz}, 2 \mathrm{H}), 1.67(\mathrm{dt}, J=$ 
12.4, $3.6 \mathrm{~Hz}, 1 \mathrm{H}), 1.33-1.44(\mathrm{~m}, 2 \mathrm{H}), 1.25(\mathrm{tt}, J=12.0,3.6 \mathrm{~Hz}, 1 \mathrm{H}), 1.11-1.21(\mathrm{~m}, 2 \mathrm{H}) ;{ }^{13} \mathrm{C}$ $\mathrm{NMR}\left(\mathrm{CDCl}_{3}\right) \delta 160.74,148.67,129.82,106.24,101.61,98.98,54.90,51.57,33.32,25.82,24.92$.<smiles>COc1cccc(NC2CCCCC2)c1</smiles>

$\boldsymbol{N}$-(Cyclohexylamino)-m-anisidine. ${ }^{8}$ (Table 3, entry 22). The general procedure conducted with 3iodoanisole $(0.234 \mathrm{~g}, 1.00 \mathrm{mmol})$, cyclohexylamine $(0.119 \mathrm{~g}, 1.20 \mathrm{mmol}), \mathrm{Pd}(\mathrm{OAc})_{2}$ and CyPF- $t$-Bu $\left(50.0 \mu \mathrm{L}\right.$ from stock solution $\left.\mathbf{A}, 5.0 \times 10^{-4} \mathrm{mmol}\right)$ and sodium tert-butoxide $(0.135 \mathrm{~g}, 1.40 \mathrm{mmol})$ gave $196.7 \mathrm{mg}(94 \%)$ of $\mathrm{N}$-(cyclohexylamino)- $\mathrm{m}$-anisidine as a pale yellow liquid. $\left({ }^{1} \mathrm{H}\right.$ and ${ }^{13} \mathrm{C}$ NMR spectra are the same as those for the product in Table 3, entry 21.)<smiles>COc1cccc(NC(Cl)Cl)c1</smiles>

N-Octyl-1-aminonaphathlene. ${ }^{9}$ (Table 3, entry 23). The general procedure conducted with 1iodonaphathlene $(0.254 \mathrm{~g}, 1.00 \mathrm{mmol})$, octylamine $(0.155 \mathrm{~g}, 1.20 \mathrm{mmol}), \mathrm{Pd}(\mathrm{OAc})_{2}$ and CyPF- $t$-Bu $\left(50.0 \mu \mathrm{L}\right.$ from stock solution $\left.\mathbf{A}, 5.0 \times 10^{-4} \mathrm{mmol}\right)$ and sodium tert-butoxide $(0.135 \mathrm{~g}, 1.40 \mathrm{mmol})$ gave $249.9 \mathrm{mg}(98 \%)$ of $\mathrm{N}$-octyl-1-aminonaphathlene as a pale yellow solid. ${ }^{1} \mathrm{H} \mathrm{NMR}\left(\mathrm{CDCl}_{3}\right) \delta 7.81-7.84$ $(\mathrm{m}, 2 \mathrm{H}), 7.42-7.50(\mathrm{~m}, 2 \mathrm{H}), 7.39(\mathrm{t}, J=8.0 \mathrm{~Hz}, 1 \mathrm{H}), 7.26(\mathrm{~d}, J=8.4 \mathrm{~Hz}, 1 \mathrm{H}), 6.64(\mathrm{~d}, J=7.6 \mathrm{~Hz}, 1$ $\mathrm{H}), 4.33$ (s, br, $1 \mathrm{H}), 3.28$ (t, $J=7.2 \mathrm{~Hz}, 2 \mathrm{H}), 1.78$ (quint, $J=7.6 \mathrm{~Hz}, 2 \mathrm{H}), 1.35-1.55(\mathrm{~m}, 10 \mathrm{H}), 0.95$ $(\mathrm{t}, J=7.2 \mathrm{~Hz}, 3 \mathrm{H}) ;{ }^{13} \mathrm{C} \mathrm{NMR}\left(\mathrm{CDCl}_{3}\right) \delta 143.60,134.26,128.62,126.65,125.59,124.52,123.27$, $119.74,116.98,104.11,44.20,31.85,29.47,29.41,29.28,27.36,22.68,14.12$.

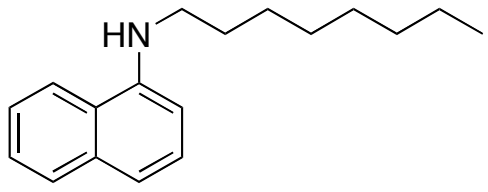

$N$-(isobutyl)-2-isopropylaniline. (Table 3, entry 24). The general procedure A conducted with 1bromo-2-isopropylbenzene (0.199 g, $1.00 \mathrm{mmol})$, iso-butylamine (87.8 $\mathrm{mg}, 1.20 \mathrm{mmol}), \mathrm{Pd}(\mathrm{OAc})_{2}$ and CyPF- $t$-Bu $\left(50.0 \mu \mathrm{L}\right.$ from stock solution $\left.\mathbf{A}, 5.0 \times 10^{-4} \mathrm{mmol}\right)$ gave $181.2 \mathrm{mg}(95 \%)$ of N-(isobutyl)-2isopropylaniline as a pale yellow liquid. . ${ }^{1} \mathrm{H} \mathrm{NMR}\left(\mathrm{CDCl}_{3}\right) \delta 7.21(\mathrm{dd}, J=7.5,1.5 \mathrm{~Hz}, 1 \mathrm{H}), 7.17$ (td, $J=7.5,1.5 \mathrm{~Hz}, 1 \mathrm{H}), 6.78(\mathrm{td}, J=7.5,1.0 \mathrm{~Hz}, 1 \mathrm{H}), 6.62(\mathrm{~d}, J=7.5 \mathrm{~Hz}, 1 \mathrm{H}), 3.79(\mathrm{~s}, \mathrm{~b}, 1 \mathrm{H}), 3.03(\mathrm{~d}$, $J=6.5 \mathrm{~Hz}, 2 \mathrm{H}$ ), 2.92 (hept, $J=7.0 \mathrm{~Hz}, 1 \mathrm{H}$ ), 2.01 (hept, $J=6.5 \mathrm{~Hz}, 1 \mathrm{H}), 1.33(\mathrm{~d}, J=7.0 \mathrm{~Hz}, 6 \mathrm{H}$ ), $1.08(\mathrm{~d}, J=6.5 \mathrm{~Hz}, 6 \mathrm{H}) ;{ }^{13} \mathrm{C} \mathrm{NMR}\left(\mathrm{CDCl}_{3}\right) \delta 145.05,131.67,126.70,124.80,116.76,110.15,51.83$, $27.89,27.18,22.18,20.58$.

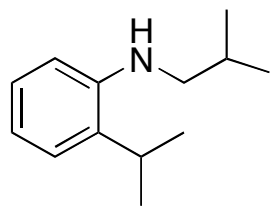

$\boldsymbol{N}$-(2, 6-Xylyl)octylamine. (Table 3, entry 25). The general procedure A conducted with 2, 6dimethyl-1-chloro-benzene $(0.141 \mathrm{~g}, 1.00 \mathrm{mmol})$, octylamine $(0.155 \mathrm{~g}, 1.20 \mathrm{mmol}), \mathrm{Pd}(\mathrm{OAc})_{2}$ and CyPF- $t$-Bu $\left(100.0 \mu \mathrm{L}\right.$ from stock solution A, $\left.1.0 \times 10^{-3} \mathrm{mmol}\right)$ gave $226.3 \mathrm{mg}(97 \%)$ of $N-(2,6-$ xylyl)octylamine as a yellow liquid. ${ }^{1} \mathrm{H} \mathrm{NMR}\left(\mathrm{CDCl}_{3}\right) \delta 7.10(\mathrm{~d}, J=7.6 \mathrm{~Hz}, 2 \mathrm{H}), 6.92(\mathrm{t}, J=7.6 \mathrm{~Hz}, 1$ $\mathrm{H}$ ), 3.10 (t, $J=7.2 \mathrm{~Hz}, 2 \mathrm{H}$ ), 3.07 (s, b, $1 \mathrm{H}$ ), 2.41 (s, $6 \mathrm{H}$ ), 1.71 (quint, $J=7.2 \mathrm{~Hz}, 2 \mathrm{H}$ ), 1.43-1.53 (m, 
$10 \mathrm{H}), 1.04(\mathrm{t}, J=6.8 \mathrm{~Hz}, 3 \mathrm{H}) ;{ }^{13} \mathrm{C} \mathrm{NMR}\left(\mathrm{CDCl}_{3}\right) \delta 146.34,128.91,128.66,121.41,48.56,31.77$, 31.16, 29.46, 29.24, 27.13, 22.60, 18.43, 14.01; Anal. Calcd. For $\mathrm{C}_{16} \mathrm{H}_{27} \mathrm{~N}$ : C, 82.34; H, 11.66; N, 6.00. Found: C, 82.16; H, 11.78; N, 5.94.<smiles>Cc1cccc(C)c1NOCCO</smiles>

N-(2, 6-Xylyl)octylamine. (Table 3, entry 26). The general procedure A conducted with 2, 6dimethyl-1-chloro-benzene $(0.185 \mathrm{~g}, 1.00 \mathrm{mmol})$, octylamine $(0.155 \mathrm{~g}, 1.20 \mathrm{mmol}), \mathrm{Pd}(\mathrm{OAc})_{2}$ and CyPF- $t$-Bu $\left(50.0 \mu \mathrm{L}\right.$ from stock solution $\left.\mathbf{A}, 5.0 \times 10^{-4} \mathrm{mmol}\right)$ gave $228.3 \mathrm{mg}(98 \%)$ of $N-(2$, 6xylyl)octylamine as a yellow liquid. $\left({ }^{1} \mathrm{H}\right.$ and ${ }^{13} \mathrm{C}$ NMR spectra are the same as those for the product in Table 3, entry 25.)<smiles>Cc1cccc(C)c1NOCCO</smiles>

$\boldsymbol{N}$-(2, 6-Xylyl)-secbutylamine. (Table 3, entry 27). The general procedure A conducted with 2, 6dimethyl-1-chloro-benzene $(0.185 \mathrm{~g}, 1.00 \mathrm{mmol})$, sec-butylamine $(87.8 \mathrm{mg}, 1.20 \mathrm{mmol}), \mathrm{Pd}(\mathrm{OAc})_{2}$ $\left(1.1 \mathrm{mg}, 5.0 \times 10^{-3} \mathrm{mmol}\right)$ and CyPF- $t$-Bu $\left(2.8 \mathrm{mg}, 5.0 \times 10^{-3} \mathrm{mmol}\right)$ gave $172.7 \mathrm{mg}(97 \%)$ of $N$ - $(2,6-$ Xylyl)-secbutylamine as a yellow liquid. ${ }^{1} \mathrm{H} \mathrm{NMR}\left(\mathrm{CDCl}_{3}\right) \delta 7.01(\mathrm{~d}, J=7.2 \mathrm{~Hz}, 2 \mathrm{H}), 6.82(\mathrm{t}, J=7.2$ $\mathrm{Hz}, 1 \mathrm{H}), 3.24$ (sext, $J=6.4 \mathrm{~Hz}, 1 \mathrm{H}), 2.82(\mathrm{~s}, 1 \mathrm{H}), 2.30(\mathrm{~s}, 6 \mathrm{H}), 1.58-1.68(\mathrm{~m}, 1 \mathrm{H}), 1.37-1.48$ (m, 1 $\mathrm{H}), 1.10(\mathrm{~d}, J=6.8 \mathrm{~Hz}, 3 \mathrm{H}), 1.00(\mathrm{t}, J=7.2 \mathrm{~Hz}, 3 \mathrm{H}) ;{ }^{13} \mathrm{C} \mathrm{NMR}\left(\mathrm{CDCl}_{3}\right) \delta 145.17,128.86$, 128.78,121.02, 53.52, 30.90, 20.80, 19.03, 10.78. Anal. Calcd. For $\mathrm{C}_{12} \mathrm{H}_{19} \mathrm{~N}$ : C, 81.30; H, 10.80; N, 7.90. Found: C, 81.37; H, 11.09; N, 8.14.<smiles>CCC(C)Nc1c(C)cccc1C</smiles>

General Procedure B for Catalytic Amination of Heteroaryl and Aryl Halides in Toluene. The reaction conditions and average yields for each reaction are shown in Table 1 and 3. A typical procedure is given for the first entry in Table 5.

$N$-Octyl-2-aminopyridine. (Table 5, entry 1). A solution of $\mathrm{Pd}(\mathrm{OAc})_{2}$ and $\mathrm{CyPF}-\mathrm{t}$-Bu $(10.0 \mu \mathrm{L}$ from stock solution $\left.\mathbf{A}, 1.0 \times 10^{-4} \mathrm{mmol}\right)$ was added to a $4 \mathrm{~mL}$ vial containing 2 -chloropyridine $(0.114 \mathrm{~g}$, $1.00 \mathrm{mmol})$ and sodium tert-butoxide $(0.135 \mathrm{~g}, 1.40 \mathrm{mmol})$ in $1.0 \mathrm{~mL}$ of toluene. Octylamine $(0.155 \mathrm{~g}$, $1.20 \mathrm{mmol}$ ) was then added by syringe. The vial was sealed with a cap containing a PTFE septum, and the reaction mixture was stirred at $100{ }^{\circ} \mathrm{C}$ until 3-chloropyridine was consumed, as determined by GC. The reaction solution was directly adsorbed onto silica gel, and the product was isolated by eluting with hexane/ethyl acetate $(85 / 15)$ to give $200.9 \mathrm{mg}(98 \%)$ of $N$-octyl-2-aminopyridine as a yellow solid. $\left({ }^{1} \mathrm{H}\right.$ and ${ }^{13} \mathrm{C}$ NMR spectra are the same as those for the product in Table 1, entry 1.)

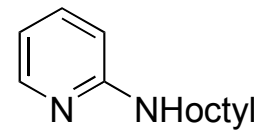


$\boldsymbol{N}$-octyl-3-aminopyridine. (Table 5, entry 2). The general procedure B conducted with 3chloropyridine $(0.114 \mathrm{~g}, 1.00 \mathrm{mmol})$, octylamine $(0.155 \mathrm{~g}, 1.20 \mathrm{mmol}), \mathrm{Pd}(\mathrm{OAc})_{2}$ and $\mathrm{CyPF}-t-\mathrm{Bu}(50.0$ $\mu \mathrm{L}$ from stock solution $\left.\mathbf{A}, 5.0 \times 10^{-4} \mathrm{mmol}\right)$ gave $210.0 \mathrm{mg}(98 \%)$ of $N$-octyl-3-aminopyridine as a white solid. ( ${ }^{1} \mathrm{H}$ and ${ }^{13} \mathrm{C}$ NMR spectra are the same as those for the product in Table 1, entry 11.)

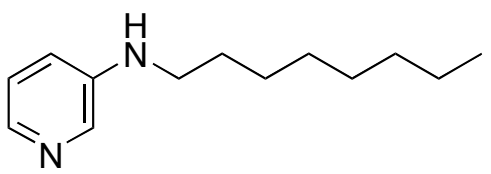

$\boldsymbol{N}$-(3-Pyridinyl)-p-toluidine. (Table 5, entry 3). The general procedure B conducted with 3chloropyridine $(0.114 \mathrm{~g}, 1.00 \mathrm{mmol}), p$-toluidine $(0.130 \mathrm{~g}, 1.20 \mathrm{mmol}), \mathrm{Pd}(\mathrm{OAc})_{2}$ and $\mathrm{CyPF}-t-\mathrm{Bu}$ $\left(100.0 \mu \mathrm{L}\right.$ from stock solution $\left.\mathbf{A}, 1.0 \times 10^{-3} \mathrm{mmol}\right)$ gave $121.1 \mathrm{mg}(66 \%)$ of $N$-(3-pyridyl)-p-toluidine as a yellow solid. ${ }^{1} \mathrm{H}$ NMR $\left(\mathrm{CDCl}_{3}\right) \delta 8.34(\mathrm{~s}, 1 \mathrm{H}), 8.12(\mathrm{~d}, J=4.4 \mathrm{~Hz}, 1 \mathrm{H}), 7.35(\mathrm{t}, J=7.6 \mathrm{~Hz}, 1 \mathrm{H})$, 7.10-7.19 (m, $3 \mathrm{H}), 7.02(\mathrm{~d}, J=8.0 \mathrm{~Hz}, 2 \mathrm{H}), 5.75(\mathrm{~s}, \mathrm{~b}, 1 \mathrm{H}), 2.33(\mathrm{~s}, 3 \mathrm{H}) ;{ }^{13} \mathrm{C} \mathrm{NMR}\left(\mathrm{CDCl}_{3}\right) \delta$ $140.79,140.42,139.10,138.87,131.46,129.81,123.56,121.97,119.02,20.54$; Anal. Calcd. For $\mathrm{C}_{12} \mathrm{H}_{12} \mathrm{~N}_{2}:$ C, 78.23; H, 6.57; N, 15.21. Found: C, 78.19; H, 6.61; N, 15.01.

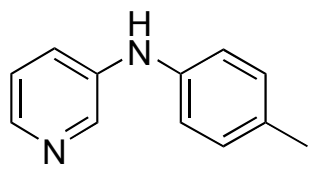

$N$-Phenyloctylamine. ${ }^{10}$ (Table 5, entry 4). The general procedure B conducted with phenyl chloride $(0.113 \mathrm{~g}, 1.00 \mathrm{mmol})$, octylamine $(0.155 \mathrm{~g}, 1.20 \mathrm{mmol}), \mathrm{Pd}(\mathrm{OAc})_{2}$ and $\mathrm{CyPF}-t-\mathrm{Bu}(50.0 \mu \mathrm{L}$ from stock solution A, $\left.5.0 \times 10^{-4} \mathrm{mmol}\right)$ gave $203.8 \mathrm{mg}(99 \%)$ of $N$-phenyloctylamine as a colorless liquid. ${ }^{1} \mathrm{H}$ NMR $\left(\mathrm{CDCl}_{3}\right) \delta 7.09(\mathrm{tt}, J=7.2,2.0 \mathrm{~Hz}, 2 \mathrm{H}), 6.60(\mathrm{tt}, J=7.2,1.0 \mathrm{~Hz}, 1 \mathrm{H}), 6.50(\mathrm{dd}, J=7.2,2.0 \mathrm{~Hz}$, $2 \mathrm{H}), 3.47(\mathrm{~s}, \mathrm{~b}, 1 \mathrm{H}), 3.00(\mathrm{t}, J=7.2 \mathrm{~Hz}, 2 \mathrm{H}), 1.52$ (quint, $J=7.2 \mathrm{~Hz}, 2 \mathrm{H}), 1.21-1.32(\mathrm{~m}, 10 \mathrm{H}), 0.82$ $(\mathrm{t}, J=6.8 \mathrm{~Hz}, 3 \mathrm{H}) ;{ }^{13} \mathrm{C} \mathrm{NMR}\left(\mathrm{CDCl}_{3}\right) \delta 148.45,129.11,116.94,112.57,43.90,31.79,29.51,29.39$, 29.23, 27.14, 22.62, 14.07.

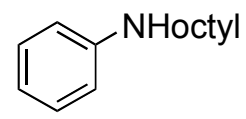

$N$-p-Tolyl-iso-butylamine. ${ }^{7}$ (Table 5, entry 5). The general procedure B conducted with 4chlorotoluene $(0.127 \mathrm{~g}, 1.00 \mathrm{mmol})$, iso-butylamine $(87.8 \mathrm{mg}, 1.20 \mathrm{mmol}), \mathrm{Pd}(\mathrm{OAc})_{2}$ and $\mathrm{CyPF}-t-\mathrm{Bu}$ $\left(20.0 \mu \mathrm{L}\right.$ from stock solution $\left.\mathbf{A}, 2.0 \times 10^{-4} \mathrm{mmol}\right)$ gave $161.6 \mathrm{mg}(99 \%)$ of $N$-p-tolyl-iso-butylamine as a pale yellow liquid. $\left({ }^{1} \mathrm{H}\right.$ and ${ }^{13} \mathrm{C}$ NMR spectra are the same as those for the product in Table 3 , entry 9.)

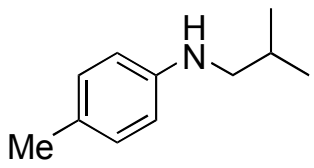

$\boldsymbol{N}$-Octyl-2-aminopyridine. (Table 5, entry 6). The general procedure B conducted with 2bromopyridine $(0.158 \mathrm{~g}, 1.00 \mathrm{mmol})$, octylamine $(0.155 \mathrm{~g}, 1.20 \mathrm{mmol}), \mathrm{Pd}(\mathrm{OAc})_{2}$ and $\mathrm{CyPF}-t$-Bu $\left(10.0 \mu \mathrm{L}\right.$ from stock solution $\left.\mathbf{A}, 1.0 \times 10^{-4} \mathrm{mmol}\right)$ gave $188.6 \mathrm{mg}(92 \%)$ of $N$-octyl-2-aminopyridine as a yellow solid. ( ${ }^{1} \mathrm{H}$ and ${ }^{13} \mathrm{C}$ NMR spectra are the same as those for the product in Table 1, entry 1.) 


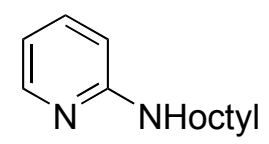

$\boldsymbol{N}$-Benzyl-3-aminopyridine. (Table 5, entry 7). The general procedure B conducted with 3chloropyridine $(0.114 \mathrm{~g}, 1.00 \mathrm{mmol})$, benzylamine $(0.129 \mathrm{~g}, 1.20 \mathrm{mmol}), \mathrm{Pd}(\mathrm{OAc})_{2}$ and CyPF- $t$-Bu $\left(10.0 \mu \mathrm{L}\right.$ from stock solution $\left.\mathbf{A}, 1.0 \times 10^{-4} \mathrm{mmol}\right)$ gave $182.2 \mathrm{mg}(99 \%)$ of $N$-benzyl-3-aminopyridine as a yellow solid. $\left({ }^{1} \mathrm{H}\right.$ and ${ }^{13} \mathrm{C}$ NMR spectra are the same as those for the product in Table 1, entry 14 .)<smiles>BrCc1ccccc1</smiles>

$N$-Benzylaniline. (Table 5, entry 8). The general procedure B conducted with phenyl bromide $(0.157$ $\mathrm{g}, 1.00 \mathrm{mmol})$, benzylamine $(0.129 \mathrm{~g}, 1.20 \mathrm{mmol}), \mathrm{Pd}(\mathrm{OAc})_{2}$ and CyPF- $t$-Bu $(5.0 \mu \mathrm{L}$ from stock solution B, $\left.5.0 \times 10^{-5} \mathrm{mmol}\right)$ gave $181.8 \mathrm{mg}(99 \%)$ of $N$-Benzylaniline as a white solid. $\left({ }^{1} \mathrm{H}\right.$ and ${ }^{13} \mathrm{C}$ NMR spectra are the same as those for the product in Table 3, entry 3.)<smiles>c1ccc(CNc2ccccc2)cc1</smiles>

$\mathbf{N}$-iso-Butyl-2-aminopyridine. (Table 5, entry 9). The general procedure B conducted with 3iodopyridine $(0.125 \mathrm{~g}, 1.00 \mathrm{mmol})$, iso-butylamine $(87.8 \mathrm{mg}, 1.20 \mathrm{mmol}), \mathrm{Pd}(\mathrm{OAc})_{2}$ and CyPF- $t$-Bu $\left(200.0 \mu \mathrm{L}\right.$ from stock solution $\left.\mathbf{A}, 2.0 \times 10^{-3} \mathrm{mmol}\right)$ and sodium tert-butoxide $(0.135 \mathrm{~g}, 1.40 \mathrm{mmol})$ gave $148.5 \mathrm{mg}(99 \%)$ of $\mathrm{N}$-iso-butyl-2-aminopyridine as a colorless liquid. $\left({ }^{1} \mathrm{H}\right.$ and ${ }^{13} \mathrm{C}$ NMR spectra are the same as those for the product in Table 1, entry 17.)<smiles>CC(C)CNc1cccnc1</smiles>

$N$-(Cyclohexylamino)-o-toluidine. ${ }^{11}$ (Table 5, entry 10). The general procedure B conducted with 2iodotoluene $(0.218 \mathrm{~g}, 1.00 \mathrm{mmol})$, cyclohexylamine $(0.119 \mathrm{~g}, 1.20 \mathrm{mmol}), \mathrm{Pd}(\mathrm{OAc})_{2}$ and CyPF- $t$-Bu $\left(50.0 \mu \mathrm{L}\right.$ from stock solution $\left.\mathbf{A}, 5.0 \times 10^{-4} \mathrm{mmol}\right)$ and sodium tert-butoxide $(0.135 \mathrm{~g}, 1.40 \mathrm{mmol})$ gave $175.0 \mathrm{mg}(93 \%)$ of $\mathrm{N}$-(cyclohexylamino)-o-toluidine as a yellow solid. ${ }^{1} \mathrm{H} \mathrm{NMR}\left(\mathrm{CDCl}_{3}\right) \delta 6.95-7.04$ (m, $2 \mathrm{H}), 6.52-6.56(\mathrm{~m}, 2 \mathrm{H}), 3.21-3.26(\mathrm{~m}, 2 \mathrm{H}), 2.04$ (s, $3 \mathrm{H}), 1.97-2.04(\mathrm{~m}, 2 \mathrm{H}), 1.56-1.71(\mathrm{~m}, 3 \mathrm{H})$, 1.08-1.34 (m, $5 \mathrm{H}) ;{ }^{13} \mathrm{C} \mathrm{NMR}\left(\mathrm{CDCl}_{3}\right) \delta 145.18,135.16,126.96,121.46,116.17,110.07,51.38,33.53$, $25.93,24.95,17.46$.

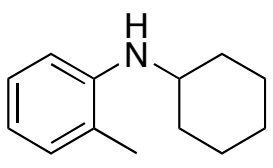

N-2-pyridyl-p-toluidine. (Table 6, entry 1). The general procedure A conducted with 2chloropyridine $(0.114 \mathrm{~g}, 1.00 \mathrm{mmol}), p$-toluidine $(0.129 \mathrm{~g}, 1.20 \mathrm{mmol}), \mathrm{Pd}(\mathrm{OAc})_{2}$ and CyPF- $t$-Bu $\left(100.0 \mu \mathrm{L}\right.$ from stock solution $\left.\mathbf{A}, 1.0 \times 10^{-3} \mathrm{mmol}\right)$ gave $158.8 \mathrm{mg}(86 \%)$ of $N$-2-pyridyl-p-toluidine as 
a white solid. ${ }^{1} \mathrm{H}$ NMR $\left(\mathrm{CDCl}_{3}\right) \delta 8.18(\mathrm{dd}, J=5.2,1.2 \mathrm{~Hz}, 1 \mathrm{H}), 7.47$ (ddd, $J=8.6,6.6,1.6 \mathrm{~Hz}, 1$ H), $7.21(\mathrm{~d}, J=8.4 \mathrm{~Hz}, 2 \mathrm{H}), 7.15(\mathrm{~d}, J=8.4 \mathrm{~Hz}, 2 \mathrm{H}), 6.83(\mathrm{~d}, J=8.4 \mathrm{~Hz}, 1 \mathrm{H}), 6.70(\mathrm{dd}, J=6.6,5.4$ $\mathrm{Hz}, 1 \mathrm{H}), 6.85$ (s, br, $1 \mathrm{H}), 2.34(\mathrm{~s}, 3 \mathrm{H}) ;{ }^{13} \mathrm{C} \mathrm{NMR}\left(\mathrm{CDCl}_{3}\right) \delta 156.73,148.02,137.83,137.59,132.47$, 129.68, 121.21, 114.14, 107.45, 20.71. Anal. Calcd. For $\mathrm{C}_{12} \mathrm{H}_{12} \mathrm{~N}_{2}$ : C, 78.23; H, 6.57; N, 15.21. Found: C, 73.08; H, 6.50; N, 15.14.<smiles>Cc1ccc(Nc2ccccn2)cc1</smiles>

$\boldsymbol{N}$-2-pyridyl-p-toluidine. (Table 6, entry 2). The general procedure A conducted with 2bromopyridine $(0.158 \mathrm{~g}, 1.00 \mathrm{mmol})$, $p$-toluidine $(0.129 \mathrm{~g}, 1.20 \mathrm{mmol}), \mathrm{Pd}(\mathrm{OAc})_{2}$ and $\mathrm{CyPF}-t-\mathrm{Bu}(50.0$ $\mu \mathrm{L}$ from stock solution $\left.\mathbf{A}, 5.0 \times 10^{-4} \mathrm{mmol}\right)$ gave $175.4 \mathrm{mg}(95 \%)$ of $N$-2-pyridyl-p-toluidine as a white solid. $\left({ }^{1} \mathrm{H}\right.$ and ${ }^{13} \mathrm{C}$ NMR spectra are the same as those for the product in Table 6, entry 1.$)$<smiles>Cc1ccc(Nc2ccccn2)cc1</smiles>

2- $\alpha$-Naphthylaminopyridine. ${ }^{12}$ (Table 6, entry 3). The general procedure A conducted with 2chloropyridine $(0.114 \mathrm{~g}, 1.00 \mathrm{mmol}), 1$-naphthylamine $(0.172 \mathrm{~g}, 1.20 \mathrm{mmol}), \mathrm{Pd}(\mathrm{OAc})_{2}$ and CyPF-t$\mathrm{Bu}\left(50.0 \mu \mathrm{L}\right.$ from stock solution $\left.\mathbf{A}, 5.0 \times 10^{-4} \mathrm{mmol}\right)$ gave $214.9 \mathrm{mg}$ (98\%) of 2- $\alpha-$ naphthylaminopyridine as a colorless solid. ${ }^{1} \mathrm{H} \mathrm{NMR}\left(\mathrm{CDCl}_{3}\right) \delta 8.17(\mathrm{~d}, J=5.0 \mathrm{~Hz}, 1 \mathrm{H}), 8.10(\mathrm{~d}, J=$ $8.5 \mathrm{~Hz}, 1 \mathrm{H}), 7.90(\mathrm{~d}, J=7.0 \mathrm{~Hz}, 1 \mathrm{H}), 7.72(\mathrm{~d}, J=8.5 \mathrm{~Hz}, 1 \mathrm{H}), 7.58(\mathrm{~d}, J=7.5 \mathrm{~Hz}, 1 \mathrm{H}), 7.39-7.54$ $(\mathrm{m}, 4 \mathrm{H}), 7.35(\mathrm{~s}, \mathrm{~b}, 1 \mathrm{H}), 6.69(\mathrm{t}, J=5.0 \mathrm{~Hz}, 1 \mathrm{H}), 6.62(\mathrm{~d}, J=8.0 \mathrm{~Hz}, 1 \mathrm{H}) ;{ }^{13} \mathrm{C} \mathrm{NMR}\left(\mathrm{CDCl}_{3}\right) \delta$ $157.85,148.48,137.73,136.11,134.69,129.28,128.46,126.29,126.16,125.89,125.28,122.43,120$, $52,114.59,107.63$.<smiles>c1ccc(Nc2cccc3ccccc23)nc1</smiles>

$N$-(3-Pyridinyl)-p-toluidine. (Table 6, entry 4). The general procedure A conducted with 3chloropyridine $(0.114 \mathrm{~g}, 1.00 \mathrm{mmol})$, $p$-toluidine $(0.130 \mathrm{~g}, 1.20 \mathrm{mmol}), \mathrm{Pd}(\mathrm{OAc})_{2}$ and $\mathrm{CyPF}-t-\mathrm{Bu}(50.0$ $\mu \mathrm{L}$ from stock solution $\left.\mathbf{A}, 5.0 \times 10^{-4} \mathrm{mmol}\right)$ gave $181.6 \mathrm{mg}(93 \%)$ of $N$-(3-pyridyl)-p-toluidine as a yellow solid. ${ }^{1} \mathrm{H}$ NMR $\left(\mathrm{CDCl}_{3}\right) \delta 8.34(\mathrm{~s}, 1 \mathrm{H}), 8.12(\mathrm{~d}, J=4.4 \mathrm{~Hz}, 1 \mathrm{H}), 7.35(\mathrm{t}, J=7.6 \mathrm{~Hz}, 1 \mathrm{H})$, 7.10-7.19 (m, $3 \mathrm{H}), 7.02(\mathrm{~d}, J=8.0 \mathrm{~Hz}, 2 \mathrm{H}), 5.75(\mathrm{~s}, \mathrm{~b}, 1 \mathrm{H}), 2.33(\mathrm{~s}, 3 \mathrm{H}) ;{ }^{13} \mathrm{C} \mathrm{NMR}\left(\mathrm{CDCl}_{3}\right) \delta$ $140.79,140.42,139.10,138.87,131.46,129.81,123.56,121.97,119.02,20.54$; Anal. Calcd. For $\mathrm{C}_{12} \mathrm{H}_{12} \mathrm{~N}_{2}:$ C, 78.23; H, 6.57; N, 15.21. Found: C, 78.19; H, 6.61; N, 15.01.<smiles>Cc1ccc(Nc2cccnc2)cc1</smiles>

N-3-pyridyl-o-anisidine. (Table 6, entry 5). The general procedure A conducted with 3chloropyridine $(0.114 \mathrm{~g}, 1.00 \mathrm{mmol}), o$-anisidine $(0.148 \mathrm{~g}, 1.20 \mathrm{mmol}), \mathrm{Pd}(\mathrm{OAc})_{2}$ and $\mathrm{CyPF}-t$-Bu $(5.0$ $\mu \mathrm{L}$ from stock solution $\left.\mathbf{A}, 5.0 \times 10^{-4} \mathrm{mmol}\right)$ gave $197.1 \mathrm{mg}(99 \%)$ of $N$-3-pyridyl-o-anisidine as colorless needles. ${ }^{1} \mathrm{H}$ NMR $\left(\mathrm{CDCl}_{3}\right) \delta 8.44(\mathrm{~d}, J=2.5 \mathrm{~Hz}, 1 \mathrm{H}), 8.17(\mathrm{dd}, J=4.5,1.5 \mathrm{~Hz}, 1 \mathrm{H}), 7.48$ 
(ddd, $J=8.0,2.5,1.5 \mathrm{~Hz}, 1 \mathrm{H}), 7.16-7.26(\mathrm{~m}, 1 \mathrm{H}), 7.17(\mathrm{dd}, J=8.0,4.5 \mathrm{~Hz}, 1 \mathrm{H}), 6.88-6.93(\mathrm{~m}, 3$

$\mathrm{H}), 6.16$ (s, b, $1 \mathrm{H}), 3.89$ (s, $3 \mathrm{H}) ;{ }^{13} \mathrm{C}$ NMR $\left(\mathrm{CDCl}_{3}\right) \delta 148.67,142.03,140.85,139.31,131.63,123.97$, 123.58, 121.06, 120.77, 115.03, 110.67, 55.53; Anal. Calcd. For $\mathrm{C}_{12} \mathrm{H}_{12} \mathrm{~N}_{2} \mathrm{O}: \mathrm{C}, 71.98 ; \mathrm{H}, 6.04 ; \mathrm{N}$, 13.99. Found: C, 72.05; H, 6.09; N, 13.89 .<smiles>COc1ccccc1Nc1cccnc1</smiles>

2,3'-Dipyridylamine. ${ }^{13}$ (Table 6, entry 6). The general procedure A conducted with 3-chloropyridine $(0.114 \mathrm{~g}, 1.00 \mathrm{mmol}), 2$-aminopyridine $(0.113 \mathrm{~g}, 1.20 \mathrm{mmol}), \mathrm{Pd}(\mathrm{OAc})_{2}\left(1.1 \mathrm{mg}, 5.0 \times 10^{-3} \mathrm{mmol}\right)$ and CyPF-t-Bu $\left(2.8 \mathrm{mg}, 5.0 \times 10^{-3} \mathrm{mmol}\right)$ gave $165.0 \mathrm{mg}(97 \%)$ of 2,3'-dipyridylamine as a colorless solid. ${ }^{1} \mathrm{H} \mathrm{NMR}\left(\mathrm{CDCl}_{3}\right) \delta 8.69(\mathrm{~d}, J=2.5 \mathrm{~Hz}, 1 \mathrm{H}), 8.25(\mathrm{dd}, J=4.5,1.5 \mathrm{~Hz}, 1 \mathrm{H}), 8.23(\mathrm{dd}, J=5.0,1.0 \mathrm{~Hz}$, $1 \mathrm{H}), 8.00$ (ddd, $J=8.0,2.5,1.5 \mathrm{~Hz}, 1 \mathrm{H}), 7.50-7.54(\mathrm{~m}, 1 \mathrm{H}), 7.32$ (s, b, $1 \mathrm{H}), 7.23-7.27$ (m, $1 \mathrm{H}), 6.81$ $(\mathrm{d}, J=8.0 \mathrm{~Hz}, 1 \mathrm{H}), 6.77-6.80(\mathrm{~m}, 1 \mathrm{H}) ;{ }^{13} \mathrm{C} \mathrm{NMR}\left(\mathrm{CDCl}_{3}\right) \delta 155.33,148.12,143.09,141.48,137.83$, $137.56,126.18,123.66,115.70,109.30$.<smiles>c1ccc(Nc2cccnc2)nc1</smiles>

$N$-3-pyridyl-p-anisidine. (Table 6, entry 7). The general procedure A conducted with 3bromopyridine $(0.158 \mathrm{~g}, 1.00 \mathrm{mmol}), p$-anisidine $(0.148 \mathrm{~g}, 1.20 \mathrm{mmol}), \mathrm{Pd}(\mathrm{OAc})_{2}$ and $\mathrm{CyPF}-t$-Bu (50.0 $\mu \mathrm{L}$ from stock solution A, $5.0 \times 10^{-4} \mathrm{mmol}$ ) gave $161.0 \mathrm{mg}(80 \%)$ of $N$-3-pyridyl-p-anisidine as a white solid. ${ }^{1} \mathrm{H}$ NMR $\left(\mathrm{CDCl}_{3}\right) \delta 8.22(\mathrm{~d}, J=2.8 \mathrm{~Hz}, 1 \mathrm{H}), 8.03(\mathrm{dd}, J=4.4,1.0 \mathrm{~Hz}, 1 \mathrm{H}), 7.18$ (ddd, $J$ $=8.0,2.4,1.4 \mathrm{~Hz}, 1 \mathrm{H}), 7.05-7.09(\mathrm{~m}, 1 \mathrm{H}), 7.05(\mathrm{~d}, J=8.8 \mathrm{~Hz}, 2 \mathrm{H}), 6.85(\mathrm{~d}, J=8.8 \mathrm{~Hz}, 2 \mathrm{H}), 5.84(\mathrm{~s}$, br, $1 \mathrm{H}), 3.77(\mathrm{~s}, 3 \mathrm{H}) ;{ }^{13} \mathrm{C}$ NMR $\left(\mathrm{CDCl}_{3}\right) \delta 155.73,141.70,140.33,138.17,134.32,123.64,122.55$, 121.02, 114.74, 55.49. Anal. Calcd. For $\mathrm{C}_{12} \mathrm{H}_{12} \mathrm{~N}_{2} \mathrm{O}$ : C, 71.98; H, 6.04; N, 13.99. Found: C, 71.93; H, $6.08 ; \mathrm{N}, 14.03$.

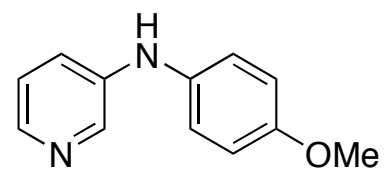

$N$-3-pyridyl-p-anisidine. (Table 6, entry 8). The general procedure A conducted with 3-iodopyridine $(0.205 \mathrm{~g}, 1.00 \mathrm{mmol}), p$-anisidine $(0.148 \mathrm{~g}, 1.20 \mathrm{mmol}), \mathrm{Pd}(\mathrm{OAc})_{2}$ and CyPF- $t$-Bu $(50.0 \mu \mathrm{L}$ from stock solution A, $5.0 \times 10^{-4} \mathrm{mmol}$ ) gave $195.2 \mathrm{mg}(97 \%)$ of $N$-3-pyridyl-p-anisidine as a white solid. $\left({ }^{1} \mathrm{H}\right.$ and ${ }^{13} \mathrm{C}$ NMR spectra are the same as those for the product in Table 6, entry 7.)<smiles>COc1ccc(Nc2cccnc2)cc1</smiles>

$\mathrm{N}$-3-pyridyl-2-pyrimidinamine. ${ }^{14}$ (Table 6, entry 9). The general procedure A conducted with 3bromopyridine ( $0.158 \mathrm{~g}, 1.00 \mathrm{mmol}), 2$-aminopyrimidine ( $0.113 \mathrm{~g}, 1.20 \mathrm{mmol}), \mathrm{Pd}(\mathrm{OAc})_{2}(2.2 \mathrm{mg}, 1.0$ $\left.\times 10^{-2} \mathrm{mmol}\right)$ and CyPF-t-Bu $\left(5.5 \mathrm{mg}, 1.0 \times 10^{-2} \mathrm{mmol}\right)$ gave $165.1 \mathrm{mg}(96 \%)$ of $\mathrm{N}$-3-pyridyl-2pyrimidinamine as a white solid. ${ }^{1} \mathrm{H}$ NMR $\left(\mathrm{CDCl}_{3}\right) \delta 8.70(\mathrm{~d}, J=2.0 \mathrm{~Hz}, 1 \mathrm{H}), 8.42(\mathrm{~d}, J=4.5 \mathrm{~Hz}, 2$ 
H), $8.26(\mathrm{~d}, J=4.5 \mathrm{~Hz}, 1 \mathrm{H}), 8.24(\mathrm{ddd}, J=8.5,2.5,1.5 \mathrm{~Hz}, 2 \mathrm{H}), 7.24(\mathrm{dd}, J=8.0,5.0 \mathrm{~Hz}, 1 \mathrm{H})$, $6.74(\mathrm{t}, J=5.0 \mathrm{~Hz}, 1 \mathrm{H}) ;{ }^{13} \mathrm{C} \mathrm{NMR}\left(\mathrm{CDCl}_{3}\right) \delta 160.01,158.01,143.48,141.18,136.40,126.26,123.41$, 113.12 .<smiles>c1cnc(Nc2cccnc2)nc1</smiles>

N-3-pyridyl-2-pyrazinamine. (Table 6, entry 10). The general procedure A conducted with 3bromopyridine $(0.158 \mathrm{~g}, 1.00 \mathrm{mmol})$, 2-aminopyrazine $(0.113 \mathrm{~g}, 1.20 \mathrm{mmol}), \mathrm{Pd}(\mathrm{OAc})_{2}(2.2 \mathrm{mg}, 1.0 \times$ $\left.10^{-2} \mathrm{mmol}\right)$ and CyPF-t-Bu $\left(5.5 \mathrm{mg}, 1.0 \times 10^{-2} \mathrm{mmol}\right)$ gave $158.1 \mathrm{mg}(92 \%)$ of $\mathrm{N}$-3-pyridyl-2pyrazinamine as a white solid. ${ }^{1} \mathrm{H} \mathrm{NMR}\left(\mathrm{CD}_{3} \mathrm{OD}\right) \delta 8.75(\mathrm{~d}, J=2.5 \mathrm{~Hz}, 1 \mathrm{H}), 8.42$ (ddd, $J=8.0,2.5$, $1.0 \mathrm{~Hz}, 1 \mathrm{H}), 8.07-8.08(\mathrm{~m}, 2 \mathrm{H}), 8.04(\mathrm{dd}, J=4.5,1.0 \mathrm{~Hz}, 1 \mathrm{H}), 7.84(\mathrm{~d}, J=4.5 \mathrm{~Hz}, 1 \mathrm{H}), 7.27(\mathrm{dd}, J$ $=8.5,4.5 \mathrm{~Hz}, 1 \mathrm{H}) ;{ }^{13} \mathrm{C} \mathrm{NMR}\left(\mathrm{CD}_{3} \mathrm{OD}\right) \delta 153.92,142.80,142.69,140.70,139.47,135.77,135.08$, $127.24,125.15$.<smiles>c1cncc(Nc2cnccn2)c1</smiles>

$N$-pyrimidyl-p-toluidine. (Table 6, entry 11). The general procedure A conducted with 5bromopyrimidine $(0.158 \mathrm{~g}, 1.00 \mathrm{mmol}), p$-toluidine $(0.128 \mathrm{~g}, 1.20 \mathrm{mmol})$, potassium phosphate $(0.254$ g, $1.40 \mathrm{mmol}), \mathrm{Pd}(\mathrm{OAc})_{2}(2.2 \mathrm{mg}, 1.0 \mathrm{mmol})$ and CyPF-t-Bu $(5.5 \mathrm{mg}, 1.0 \mathrm{mmol})$ gave $96.5 \mathrm{mg}(52 \%)$ of $N$-pyrimidyl-p-toluidine as a colorless powder. ${ }^{1} \mathrm{H}$ NMR $\left(\mathrm{CDCl}_{3}\right) \delta 8.72(\mathrm{~s}, 1 \mathrm{H}), 8.45(\mathrm{~s}, 2 \mathrm{H}), 7.15$ $(\mathrm{d}, J=8.5 \mathrm{~Hz}, 2 \mathrm{H}), 7.03(\mathrm{~d}, J=8.5 \mathrm{~Hz}, 2 \mathrm{H}), 5.68(\mathrm{~s}, \mathrm{~b}, 1 \mathrm{H}), 2.33(\mathrm{~s}, 3 \mathrm{H}) ;{ }^{13} \mathrm{C} \mathrm{NMR}\left(\mathrm{CDCl}_{3}\right) \delta$ 150.45, 143.99, 138.99, 133.39, 130.33, 120.04, 105.29, 20.76; Anal. Calcd. For $\mathrm{C}_{11} \mathrm{H}_{11} \mathrm{~N}_{3}$ : C, 71.33; H, 5.99; N, 22.69. Found: C, 71.11; H, 5.96; N, 22.23.<smiles>Cc1ccc(Nc2cncnc2)cc1</smiles>

$\boldsymbol{N}$-(4-isoquinolinyl)-p-toluidine. (Table 6, entry 12). The general procedure A conducted with 4bromoisoquinoline $(0.104 \mathrm{~g}, 0.500 \mathrm{mmol})$, p-toluidine $(65.0 \mathrm{mg}, 0.600 \mathrm{mmol}), \mathrm{Pd}(\mathrm{OAc})_{2}$ and CyPF- $t$ $\mathrm{Bu}\left(25.0 \mu \mathrm{L}\right.$ from stock solution $\left.\mathbf{A}, 2.5 \times 10^{-4} \mathrm{mmol}\right)$ gave $98.9 \mathrm{mg}(84 \%)$ of $N$-(4-isoquinolinyl)- $p$ toluidine as a white solid. ${ }^{1} \mathrm{H}$ NMR $\left(\mathrm{CDCl}_{3}\right) \delta 8.91(\mathrm{~s}, 1 \mathrm{H}), 8.41(\mathrm{~S}, 1 \mathrm{H}), 7.94(\mathrm{~d}, J=8.4 \mathrm{~Hz}, 2 \mathrm{H})$, 7.56-7.65 (m, $2 \mathrm{H}), 7.07(\mathrm{~d}, J=8.4 \mathrm{~Hz}, 2 \mathrm{H}), 6.93(\mathrm{~d}, J=8.4 \mathrm{~Hz}, 2 \mathrm{H}), 6.04(\mathrm{~S}, 1 \mathrm{H}), 2.30(\mathrm{~S}, 3 \mathrm{H}) ;{ }^{13} \mathrm{C}$ NMR $\left(\mathrm{CDCl}_{3}\right) \delta 146.14,141.02,134.39,132.42,130.99,129.91,129.63,129.57,128.97,127.88$, 127.24, 120.96, 118.42, 20.64. Anal. Calcd. For $\mathrm{C}_{16} \mathrm{H}_{14} \mathrm{~N}_{2}$ : C, 82.02; H, 6.02; N, 11.96. Found: C, $81.69 ; \mathrm{H}, 6.13 ; \mathrm{N}, 11.88$.

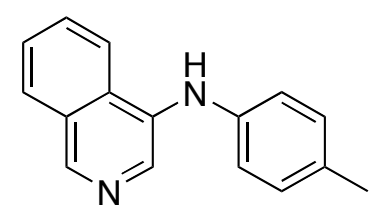


$N$-Phenyl-p-toluidine ${ }^{15}$. (Table 6, entry 13). The general procedure A conducted with phenyl chloride $(0.113 \mathrm{~g}, 1.00 \mathrm{mmol}), p$-toluidine $(0.129 \mathrm{~g}, 1.20 \mathrm{mmol}), \mathrm{Pd}(\mathrm{OAc})_{2}$ and CyPF- $t$-Bu $(50.0 \mu \mathrm{L}$ from stock solution $\left.\mathbf{A}, 5.0 \times 10^{-4} \mathrm{mmol}\right)$ gave $182.3 \mathrm{mg}(99 \%)$ of $N$-phenyl-p-toluidine as a white solid. ${ }^{1} \mathrm{H} \mathrm{NMR}\left(\mathrm{CDCl}_{3}\right) \delta 7.28(\mathrm{t}, J=8.4 \mathrm{~Hz}, 2 \mathrm{H}), 7.13(\mathrm{~d}, J=8.0 \mathrm{~Hz}, 2 \mathrm{H}), 7.03-7.06(\mathrm{~m}, 4 \mathrm{H}), 6.92(\mathrm{t}, J=$ $7.2 \mathrm{~Hz}, 1 \mathrm{H}), 5.62(\mathrm{~s}, \mathrm{br}, 1 \mathrm{H}), 2.35(\mathrm{~s}, 3 \mathrm{H}) ;{ }^{13} \mathrm{C} \mathrm{NMR}\left(\mathrm{CDCl}_{3}\right) \delta 143.84,140.18,130.83,129.79$, $129.24,120.21,118.82,116.76,20.65$.<smiles>Cc1ccc(Nc2ccccc2)cc1</smiles>

$N$-Phenyl-p-toluidine ${ }^{15}$. (Table 6, entry 14). The general procedure A conducted with phenyl bromide $(0.157 \mathrm{~g}, 1.00 \mathrm{mmol}), p$-toluidine $(0.129 \mathrm{~g}, 1.20 \mathrm{mmol}), \mathrm{Pd}(\mathrm{OAc})_{2}$ and CyPF- $t$-Bu $(5.0 \mu \mathrm{L}$ from stock solution A, $\left.5.0 \times 10^{-5} \mathrm{mmol}\right)$ gave $174.9 \mathrm{mg}(95 \%)$ of $N$-phenyl-p-toluidine as a white solid. $\left({ }^{1} \mathrm{H}\right.$ and ${ }^{13} \mathrm{C}$ NMR spectra are the same as those for the product in Table 6, entry 13.)<smiles>Cc1ccc(Nc2ccccc2)cc1</smiles>

$\boldsymbol{N}$-(2-Pyridyl)-aniline ${ }^{16}$. (Table 6, entry 15). The general procedure A conducted with phenyl bromide $(0.157 \mathrm{~g}, 1.00 \mathrm{mmol}), 2$-aminopyridine $(0.113 \mathrm{~g}, 1.20 \mathrm{mmol}), \mathrm{Pd}(\mathrm{OAc})_{2}\left(2.2 \mathrm{mg}, 1.0 \times 10^{-2} \mathrm{mmol}\right)$ and CyPF- $t$-Bu $\left(5.5 \mathrm{mg}, 1.0 \times 10^{-2} \mathrm{mmol}\right)$ gave $138.0 \mathrm{mg}(81 \%)$ of $N$-(2-pyridyl)-aniline as a white solid. ${ }^{1} \mathrm{H} \mathrm{NMR}\left(\mathrm{CDCl}_{3}\right) \delta 8.19(\mathrm{~d}, J=8.0 \mathrm{~Hz}, 1 \mathrm{H}), 7.44-7.50(\mathrm{~m}, 1 \mathrm{H}), 7.33-7.34(\mathrm{~m}, 2 \mathrm{H}), 7.30-7.32(\mathrm{~m}, 2$ $\mathrm{H}), 7.02-7.07(\mathrm{~m}, 2 \mathrm{H}), 6.88(\mathrm{~d}, J=8.0 \mathrm{~Hz}, 1 \mathrm{H}), 6.71(\mathrm{t}, J=7.0 \mathrm{~Hz}, 1 \mathrm{H}) ;{ }^{13} \mathrm{C} \mathrm{NMR}\left(\mathrm{CDCl}_{3}\right) \delta 155.99$, $148.32,140.42,137.70,129.26,122.78,120.34,114.95,108.14$.<smiles>c1ccc(Nc2ccccn2)cc1</smiles>

$\boldsymbol{N}$-Phenylaminobenzothiazole. (Table 6, entry 16). The general procedure A conducted with phenyl bromide $(0.157 \mathrm{~g}, 1.00 \mathrm{mmol}), 2$-aminobenzothiazole $(0.180 \mathrm{~g}, 1.20 \mathrm{mmol}), \mathrm{Pd}(\mathrm{OAc})_{2}(2.2 \mathrm{mg}, 1.0 \times$ $\left.10^{-2} \mathrm{mmol}\right)$ and CyPF-t-Bu $\left(5.5 \mathrm{mg}, 1.0 \times 10^{-2} \mathrm{mmol}\right)$ gave $139.6 \mathrm{mg}(62 \%)$ of $\mathrm{N}-$ phenylaminobenzothiazole. as a white solid. ${ }^{1} \mathrm{H}$ NMR $\left(\mathrm{CDCl}_{3}\right) \delta 7.58(\mathrm{dd}, J=8.0,1.5 \mathrm{~Hz}, 1 \mathrm{H}), 7.49$ $(\mathrm{td}, J=8.0,1.5 \mathrm{~Hz}, 1 \mathrm{H}), 7.34(\mathrm{dd}, J=8.0,1.0 \mathrm{~Hz}, 1 \mathrm{H}), 7.25(\mathrm{t}, J=7.5 \mathrm{~Hz}, 2 \mathrm{H}), 7.17(\mathrm{t}, J=7.5 \mathrm{~Hz}, 1$ $\mathrm{H}), 7.12(\mathrm{td}, J=8.0,1.0 \mathrm{~Hz}, 1 \mathrm{H}), 7.03(\mathrm{~d}, J=7.5 \mathrm{~Hz}, 2 \mathrm{H}), 6.95-7.05(\mathrm{~s}, \mathrm{br}, 1 \mathrm{H}) ;{ }^{13} \mathrm{C} \mathrm{NMR}\left(\mathrm{CDCl}_{3}\right) \delta$ 139.67, 137.51, 134.87, 131.73, 129.38, 126.85, 126.46, 124.09, 117.24, 115.17, 110.04. Anal. Calcd. For $\mathrm{C}_{13} \mathrm{H}_{10} \mathrm{~N}_{2} \mathrm{~S}$ : C, 69.00; H, 4.45; N, 12.38. Found: C, 69.02; H, 4.49; N, 12.38 .

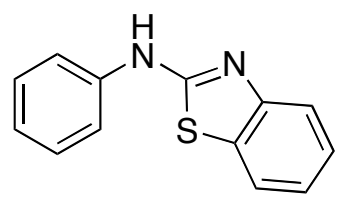

$\boldsymbol{N}$-p-tolyl-p-anisidine. ${ }^{17}$ (Table 6, entry 17). The general procedure A conducted with $p$-tolyl chloride $(0.127 \mathrm{~g}, 1.00 \mathrm{mmol}), p$-anisidine $(0.148 \mathrm{~g}, 1.20 \mathrm{mmol}), \mathrm{Pd}(\mathrm{OAc})_{2}$ and $\mathrm{CyPF}-t-\mathrm{Bu}(50.0 \mu \mathrm{L}$ from stock solution A, $\left.5.0 \times 10^{-4} \mathrm{mmol}\right)$ gave $210.6 \mathrm{mg}(99 \%)$ of $N$-p-tolyl- $p$-anisidine as a white solid. ${ }^{1} \mathrm{H}$ NMR $\left(\mathrm{CDCl}_{3}\right) \delta 7.05(\mathrm{~d}, J=8.4 \mathrm{~Hz}, 2 \mathrm{H}), 7.03(\mathrm{~d}, J=7.6 \mathrm{~Hz}, 2 \mathrm{H}), 6.84-6.88(\mathrm{~m}, 4 \mathrm{H}), 5.40(\mathrm{~s}, \mathrm{br}, 1 \mathrm{H})$, 
$3.80(\mathrm{~s}, 3 \mathrm{H}), 2.27(\mathrm{~s}, 3 \mathrm{H}) ;{ }^{13} \mathrm{C} \mathrm{NMR}\left(\mathrm{CDCl}_{3}\right) \delta 154.64,142.27,136.50,129.72,129.17,120.95$, $116.43,114.55,55.48,20.49$.<smiles>COc1ccc(Nc2ccc(C)cc2)cc1</smiles>

$N$-p-tolyl-p-anisidine. (Table 6, entry 18). The general procedure A conducted with $p$-tolyl bromide $(0.171 \mathrm{~g}, 1.00 \mathrm{mmol}), p$-anisidine $(0.148 \mathrm{~g}, 1.20 \mathrm{mmol}), \mathrm{Pd}(\mathrm{OAc})_{2}$ and CyPF- $t$-Bu $(50.0 \mu \mathrm{L}$ from stock solution A, $\left.5.0 \times 10^{-4} \mathrm{mmol}\right)$ gave $206.3 \mathrm{mg}(97 \%)$ of $N$ - $p$-tolyl- $p$-anisidine as a white solid. $\left({ }^{1} \mathrm{H}\right.$ and ${ }^{13} \mathrm{C}$ NMR spectra are the same as those for the product in Table 6, entry 18.)<smiles>COc1ccc(Nc2ccc(C)cc2)cc1</smiles>

3,4'-Dimethoxydiphenylamine. ${ }^{18}$ (Table 6, entry 19). The general procedure A conducted with 3chloroanisole $(0.143 \mathrm{~g}, 1.00 \mathrm{mmol}), p$-anisidine $(0.148 \mathrm{~g}, 1.20 \mathrm{mmol}), \mathrm{Pd}(\mathrm{OAc})_{2}$ and CyPF-t-Bu $(50.0$ $\mu \mathrm{L}$ from stock solution $\left.\mathbf{A}, 5.0 \times 10^{-4} \mathrm{mmol}\right)$ gave $227.9 \mathrm{mg}(99 \%)$ of 3,4'-Dimethoxydiphenylamine as a white solid. ${ }^{1} \mathrm{H}$ NMR $\left(\mathrm{CDCl}_{3}\right) \delta 7.13(\mathrm{t}, J=8.0 \mathrm{~Hz}, 1 \mathrm{H}), 7.09(\mathrm{~d}, J=8.4 \mathrm{~Hz}, 2 \mathrm{H}), 6.87(\mathrm{~d}, J=8.4$ $\mathrm{Hz}, 2 \mathrm{H}), 6.48-6.51(\mathrm{~m}, 2 \mathrm{H}), 6.41(\mathrm{~d}, J=8.4 \mathrm{~Hz}, 1 \mathrm{H}), 5.54(\mathrm{~s}, \mathrm{br}, 1 \mathrm{H}), 3.80(\mathrm{~s}, 3 \mathrm{H}), 3.76(\mathrm{~s}, 3 \mathrm{H}) ;{ }^{13} \mathrm{C}$ NMR $\left(\mathrm{CDCl}_{3}\right) \delta 160.66,155.30,146.60,135.33,129.97,122.57,114.55,108.21,104.59,101.19$, $55.45,55.03$.<smiles>COc1ccc(Nc2cccc(OC)c2)cc1</smiles>

3,4'-Dimethoxydiphenylamine. (Table 6, entry 20). The general procedure A conducted with 3bromoanisole $(0.187 \mathrm{~g}, 1.00 \mathrm{mmol}), p$-anisidine $(0.148 \mathrm{~g}, 1.20 \mathrm{mmol}), \mathrm{Pd}(\mathrm{OAc})_{2}$ and CyPF- $t$-Bu $(50.0$ $\mu \mathrm{L}$ from stock solution A, $\left.5.0 \times 10^{-4} \mathrm{mmol}\right)$ gave $216.4 \mathrm{mg}(94 \%)$ of 3,4'-Dimethoxydiphenylamine as a white solid. ( ${ }^{1} \mathrm{H}$ and ${ }^{13} \mathrm{C}$ NMR spectra are the same as those for the product in Table 6 , entry 19.)<smiles>COc1ccc(Nc2cccc(OC)c2)cc1</smiles>

$N$-(o-Tolyl)-p-toluidine ${ }^{18}$. (Table 6, entry 21). The general procedure A conducted with $o$-tolyl bromide $(0.171 \mathrm{~g}, 1.00 \mathrm{mmol}), p$-toluidine $(0.129 \mathrm{~g}, 1.20 \mathrm{mmol}), \mathrm{Pd}(\mathrm{OAc})_{2}$ and $\mathrm{CyPF}-t-\mathrm{Bu}(50.0 \mu \mathrm{L}$ from stock solution A, $\left.5.0 \times 10^{-4} \mathrm{mmol}\right)$ gave $189.8 \mathrm{mg}(96 \%)$ of $N$-(o-Tolyl)- $p$-toluidine as a white solid. ${ }^{1} \mathrm{H} \mathrm{NMR}\left(\mathrm{CDCl}_{3}\right) \delta 7.05(\mathrm{~d}, J=8.0 \mathrm{~Hz}, 2 \mathrm{H}), 6.99(\mathrm{t}, J=6.8 \mathrm{~Hz}, 1 \mathrm{H}), 6.95(\mathrm{~d}, J=8.0 \mathrm{~Hz}, 2 \mathrm{H})$, 6.74-6.79 (m, $3 \mathrm{H}), 5.14$ (s, br, $1 \mathrm{H}), 2.18(\mathrm{~s}, 3 \mathrm{H}), 2.11(\mathrm{~s}, 3 \mathrm{H}) ;{ }^{13} \mathrm{C} \mathrm{NMR}\left(\mathrm{CDCl}_{3}\right) \delta 141.97,140.99$, $130.76,130.29,129.75,126.98,126.67,121.03,118.54,117.25,20.59,17.77$.<smiles>Cc1ccc(Nc2ccccc2C)cc1</smiles> 
$N$-(o-Tolyl)-p-toluidine ${ }^{18}$. (Table 6, entry 22). The general procedure A conducted with $o$-tolyl iodide $(0.219 \mathrm{~g}, 1.00 \mathrm{mmol}), p$-toluidine $(0.129 \mathrm{~g}, 1.20 \mathrm{mmol}), \mathrm{Pd}(\mathrm{OAc})_{2}$ and $\mathrm{CyPF}-t-\mathrm{Bu}(50.0 \mu \mathrm{L}$ from stock solution A, $\left.5.0 \times 10^{-4} \mathrm{mmol}\right)$ gave $195.7 \mathrm{mg}(99 \%)$ of $N$-(o-Tolyl)- $p$-toluidine as a white solid. $\left({ }^{1} \mathrm{H}\right.$ and ${ }^{13} \mathrm{C}$ NMR spectra are the same as those for the product in Table 6, entry 21.$)$

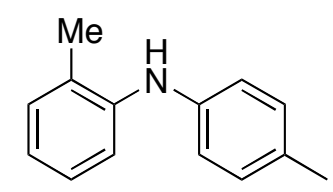

2-N-(p-Tolyl)-1,3-xylidine. (Table 6, entry 23). The general procedure A conducted with 2-bromo1,2-dimethylbenzene $(0.185 \mathrm{~g}, 1.00 \mathrm{mmol})$, $p$-toluidine $(0.129 \mathrm{~g}, 1.20 \mathrm{mmol}), \mathrm{Pd}(\mathrm{OAc})_{2}(1.1 \mathrm{mg}, 5.0 \times$ $\left.10^{-3} \mathrm{mmol}\right)$ and CyPF- $t$-Bu $\left(2.8 \mathrm{mg}, 5.0 \times 10^{-3} \mathrm{mmol}\right)$ gave $188.4 \mathrm{mg}(89 \%)$ of 2- $N$ - $(p$-Tolyl)-1,3xylidine as a white solid. ${ }^{1} \mathrm{H}$ NMR $\left(\mathrm{CDCl}_{3}\right) \delta 6.92-7.00(\mathrm{~m}, 3 \mathrm{H}), 6.85(\mathrm{~d}, J=8.4 \mathrm{~Hz}, 2 \mathrm{H}), 6.31(\mathrm{~d}, J=$ $8.0 \mathrm{~Hz}, 2 \mathrm{H}), 4.95(\mathrm{~s}, \mathrm{br}, 1 \mathrm{H}), 2.12(\mathrm{~s}, 3 \mathrm{H}), 2.09(\mathrm{~s}, 6 \mathrm{H}) ;{ }^{13} \mathrm{C} \mathrm{NMR}\left(\mathrm{CDCl}_{3}\right) \delta 143.85,138.65$, 135.50, 129.71, 128.51, 127.38, 125.39, 113.73, 20.43, 18.34. Anal. Calcd. For $\mathrm{C}_{15} \mathrm{H}_{17} \mathrm{~N}: \mathrm{C}, 85.26 ; \mathrm{H}$, $8.11 ; \mathrm{N}, 6.63$. Found: C, 85.53; H, 8.03; N, 6.76 .

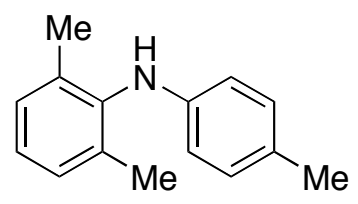

N-(p-Tolyl)-2-cyclohexylaniline. (Table 6, entry 24). The general procedure A conducted with 1bromo-2-cyclohexylbenzene $(0.239 \mathrm{~g}, 1.00 \mathrm{mmol}), p$-toluidine $(0.129 \mathrm{~g}, 1.20 \mathrm{mmol}), \mathrm{Pd}(\mathrm{OAc})_{2}$ and CyPF- $t$-Bu $\left(50.0 \mu \mathrm{L}\right.$ from stock solution $\left.\mathbf{A}, 5.0 \times 10^{-4} \mathrm{mmol}\right)$ gave $235.2 \mathrm{mg}(89 \%)$ of $N$-( $p$-Tolyl)-2cyclohexylaniline as a white solid. ${ }^{1} \mathrm{H} \mathrm{NMR}\left(\mathrm{CDCl}_{3}\right) \delta 7.30(\mathrm{dd}, J=8.0,1.2 \mathrm{~Hz}, 1 \mathrm{H}), 7.24(\mathrm{~d}, J=8.4$, $1.2 \mathrm{~Hz}, 1 \mathrm{H}), 7.14(\mathrm{td}, J=7.2,1.2 \mathrm{~Hz}, 1 \mathrm{H}), 7.10(\mathrm{~d}, J=8.0 \mathrm{~Hz}, 2 \mathrm{H}), 7.03(\mathrm{td}, J=7.2,1.2 \mathrm{~Hz}, 1 \mathrm{H})$, $6.90(\mathrm{~d}, J=8.4 \mathrm{~Hz}, 2 \mathrm{H}), 5.42(\mathrm{~s}, \mathrm{br}, 1 \mathrm{H}), 2.13-2.78(\mathrm{~m}, 1 \mathrm{H}), 2.34$ (s, $3 \mathrm{H}), 1.88-1.92$ (m, $4 \mathrm{H}), 1.79$ $1.82(\mathrm{~m}, 1 \mathrm{H}), 1.13-1.54(\mathrm{~m}, 5 \mathrm{H}) ;{ }^{13} \mathrm{C} \mathrm{NMR}\left(\mathrm{CDCl}_{3}\right) \delta 140.23,140.53,138.08,129.77,129.69$, $126.46,126.26,122.27,119.98,117.76,38.25,33.39,27.05,26.27,20.57$.

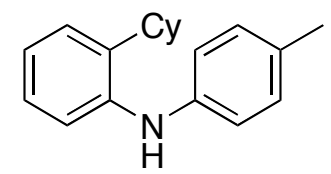

2-(N-Methylanilino)pyridine. ${ }^{11}$ (Table 7, entry 1). The general procedure A conducted with 2chloropyridine $(0.125 \mathrm{mg}, 1.10 \mathrm{mmol}), \mathrm{N}$-methylaniline $(0.106 \mathrm{mg}, 1.00 \mathrm{mmol}), \mathrm{Pd}(\mathrm{OAc})_{2}(2.2 \mathrm{mg}$, $\left.1.0 \times 10^{-2} \mathrm{mmol}\right)$ and CyPF-t-Bu $\left(5.5 \mathrm{mg}, 1.0 \times 10^{-2} \mathrm{mmol}\right)$ gave $105 \mathrm{mg}(57 \%)$ of $2-(\mathrm{N}-$ Methylanilino)pyridine as a colorless oil. ${ }^{1} \mathrm{H} \mathrm{NMR}\left(\mathrm{CDCl}_{3}\right) \delta 8.21(\mathrm{ddd}, J=5.2,1.6,0.8 \mathrm{~Hz}, 1 \mathrm{H}), 7.38$ $(\mathrm{tt}, J=8.0,2.0 \mathrm{~Hz}, 2 \mathrm{H}), 7.29$ (ddd, $J=8.4,6.8,2.0 \mathrm{~Hz}, 1 \mathrm{H}), 7.25(\mathrm{dd}, J=8.4,2.0 \mathrm{~Hz}, 2 \mathrm{H}), 7.19$ (tt, $J$ $=7.2,1.2 \mathrm{~Hz}, 1 \mathrm{H}), 6.59(\mathrm{ddd}, J=7.6,5.2,0.8 \mathrm{~Hz}, 1 \mathrm{H}), 6.51(\mathrm{dt}, J=8.4,0.8 \mathrm{~Hz}, 1 \mathrm{H}), 3.46(\mathrm{~s}, 3 \mathrm{H})$; ${ }^{13} \mathrm{C} \mathrm{NMR}\left(\mathrm{CDCl}_{3}\right) \delta 158.74,147.70,146.75,136.52,129.64,126.27,125.38,113.05,109.11,38.36$. 


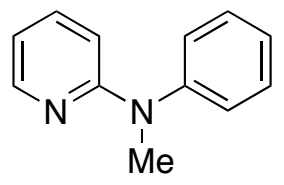

$\boldsymbol{N}$-(3-Pyridyl)morpholine. ${ }^{1}$ (Table 7, entry 2). The general procedure A conducted with 3chloropyridine $(0.114 \mathrm{mg}, 1.00 \mathrm{mmol})$, morpholine $(0.105 \mathrm{mg}, 1.20 \mathrm{mmol}), \mathrm{Pd}(\mathrm{OAc})_{2}(2.2 \mathrm{mg}, 1.0 \times$ $\left.10^{-2} \mathrm{mmol}\right)$ and CyPF- $t$-Bu $\left(5.5 \mathrm{mg}, 1.0 \times 10^{-2} \mathrm{mmol}\right)$ gave $113 \mathrm{mg}(69 \%)$ of $N$-(3-Pyridyl)morpholine as a colorless oil. ${ }^{1} \mathrm{H}$ NMR $\left(\mathrm{CDCl}_{3}\right) \delta 8.31(\mathrm{~s}, 1 \mathrm{H}), 8.13(\mathrm{t}, J=2.8 \mathrm{~Hz}, 1 \mathrm{H}), 7.19(\mathrm{~d}, J=2.4 \mathrm{~Hz}, 1 \mathrm{H})$, $7.18(\mathrm{~d}, J=1.6 \mathrm{~Hz}, 1 \mathrm{H}), 3.88(\mathrm{t}, J=4.8 \mathrm{~Hz}, 4 \mathrm{H}), 3.19(\mathrm{t}, J=4.8 \mathrm{~Hz}, 4 \mathrm{H}) ;{ }^{13} \mathrm{C} \mathrm{NMR}\left(\mathrm{CDCl}_{3}\right) \delta 147.0$, $141.2,138.4,123.7,122.3,66.8,48.7$.<smiles>c1cncc(N2CCOCC2)c1</smiles>

$\boldsymbol{N}, \boldsymbol{N}$-Di-(4'-methoxyphenyl)-4-pyridinamine. (Table 7, entry 3). The general procedure A conducted with 4-chloropyridine hydrochloride 4,4'-dimethoxyphenylamine (0.150 g, $1.00 \mathrm{mmol})$, $(0.275 \mathrm{~g}, 1.20 \mathrm{mmol}), \mathrm{Pd}(\mathrm{OAc})_{2}\left(2.2 \mathrm{mg}, 1.0 \times 10^{-2} \mathrm{mmol}\right)$ and CyPF- $t$-Bu $\left(5.5 \mathrm{mg}, 1.0 \times 10^{-2} \mathrm{mmol}\right)$ gave $152.0 \mathrm{mg}(50 \%)$ of $N, N$-Di-(4'-methoxyphenyl)-4-pyridinamine as a white solid. ${ }^{1} \mathrm{H}$ NMR $\left(\mathrm{CDCl}_{3}\right) \delta 8.16(\mathrm{~s}, \mathrm{~b}, 2 \mathrm{H}), 7.13(\mathrm{~d}, J=8.8 \mathrm{~Hz}, 4 \mathrm{H}), 7.89$ (d, J=8.8 Hz, $\left.4 \mathrm{H}\right), 6.59$ (s, b, $\left.2 \mathrm{H}\right), 3.80$ (s, $6 \mathrm{H}) ;{ }^{13} \mathrm{C} \mathrm{NMR}\left(\mathrm{CDCl}_{3}\right) \delta 157.41,154.23,149.52,137.70,128.16,114.96,110.66,55.38$. Anal. Calcd. For $\mathrm{C}_{19} \mathrm{H}_{18} \mathrm{~N}_{2} \mathrm{O}_{2}$ : C, 74.49; H, 5.92; N, 9.14. Found: C, 74.44; H, 6.02; N, 8.99.<smiles>COc1ccc(N(c2ccncc2)c2ccc(OC)cc2)cc1</smiles>

$N$-methyl- $N$-phenyl-1-isoquinolinamine (Table 7, entry 4). The general procedure A conducted with 1-chloroisoquinoline $(0.164 \mathrm{~g}, 1.00 \mathrm{mmol}), N$-methylaniline $(0.214 \mathrm{mg}, 2.00 \mathrm{mmol}), \mathrm{Pd}(\mathrm{OAc})_{2}(2.2$ $\left.\mathrm{mg}, 1.0 \times 10^{-2} \mathrm{mmol}\right)$ and CyPF- $t$-Bu $\left(5.5 \mathrm{mg}, 1.0 \times 10^{-2} \mathrm{mmol}\right)$ gave $150.3 \mathrm{mg}(64 \%)$ of $N$-methyl- $N$ phenyl-1-isoquinolinamine as a yellow solid. ${ }^{1} \mathrm{H} \mathrm{NMR}\left(\mathrm{CDCl}_{3}\right) \delta 8.20(\mathrm{~d}, J=6.0 \mathrm{~Hz}, 1 \mathrm{H}), 7.63(\mathrm{~d}, J=$ $7.6 \mathrm{~Hz}, 1 \mathrm{H}), 7.60(\mathrm{~d}, J=8.4 \mathrm{~Hz}, 1 \mathrm{H}), 7.42(\mathrm{td}, J=8.2,0.8 \mathrm{~Hz}, 1 \mathrm{H}), 7.24(\mathrm{~d}, J=5.6 \mathrm{~Hz}, 1 \mathrm{H}), 7.15$ $(\mathrm{td}, J=8.0,1.2 \mathrm{~Hz}, 1 \mathrm{H}), 7.12(\mathrm{t}, J=8.0 \mathrm{~Hz}, 2 \mathrm{H}), 6.87(\mathrm{tt}, J=7.6,1.2 \mathrm{~Hz}, 1 \mathrm{H}), 6.82(\mathrm{~d}, J=8.4 \mathrm{~Hz}, 2$ $\mathrm{H}), 3.50(\mathrm{~s}, 3 \mathrm{H}) ;{ }^{13} \mathrm{C} \mathrm{NMR}\left(\mathrm{CDCl}_{3}\right) \delta 158.38,150.68,141.11,138.19,129.48,129.18,126.87,126.68$, 126.09, 122.77, 122.13, 121.15, 116.69, 41.34. Anal. Calcd. For $\mathrm{C}_{16} \mathrm{H}_{14} \mathrm{~N}_{2}$ : C, 82.02; H, 6.02; N, 11.96. Found: C, 82.01; H, 6.10; N, 11.85 .<smiles>CN(c1ccccc1)c1nccc2ccccc12</smiles> 
$\boldsymbol{N}$-Methyldiphenylamine. ${ }^{19}$ (Table 7, entry 5). The general procedure A conducted with phenyl bromide $(0.157 \mathrm{~g}, 1.00 \mathrm{mm0l}), N$-methylaniline $(0.129 \mathrm{~g}, 1.20 \mathrm{mmol}), \mathrm{Pd}(\mathrm{OAc})_{2}$ and $\mathrm{CyPF}-t$-Bu $(50.0$ $\mu \mathrm{L}$ from stock solution $\left.\mathbf{A}, 5.0 \times 10^{-4} \mathrm{mmol}\right)$ gave $102.3 \mathrm{mg}(56 \%)$ of $N$-Methyldiphenylamine as a pale yellow liquid. ${ }^{1} \mathrm{H} \mathrm{NMR}\left(\mathrm{CDCl}_{3}\right) \delta 7.31(\mathrm{t}, J=8.5 \mathrm{~Hz}, 4 \mathrm{H}), 7.06(\mathrm{dd}, J=8.5,1.0 \mathrm{~Hz}, 4 \mathrm{H}), 6.99(\mathrm{tt}, J=$ 8.5, $1.0 \mathrm{~Hz}, 2 \mathrm{H}), 3.35$ (s, $3 \mathrm{H}) ;{ }^{13} \mathrm{C} \mathrm{NMR}\left(\mathrm{CDCl}_{3}\right) \delta 148.97,129.16,121.21,120.39,40.18$.<smiles>CN(c1ccccc1)c1ccccc1</smiles>

$N, N$-Di-butylaniline. ${ }^{20}$ (Table 7 , entry 6). The general procedure A conducted with phenyl bromide $(0.157 \mathrm{~g}, 1.00 \mathrm{~mm} 01)$, dibutylamine $(0.155 \mathrm{~g}, 1.20 \mathrm{mmol}), \mathrm{Pd}(\mathrm{OAc})_{2}$ and CyPF- $t$-Bu $\mathrm{Pd}(\mathrm{OAc})_{2}(1.1$ $\left.\mathrm{mg}, 5.0 \times 10^{-3} \mathrm{mmol}\right)$ and CyPF- $t$-Bu $\left(2.8 \mathrm{mg}, 5.0 \times 10^{-3} \mathrm{mmol}\right)$ gave $140.5 \mathrm{mg}(69 \%)$ of $N$, $N$-Dibutylaniline as a white solid. ${ }^{1} \mathrm{H}$ NMR $\left(\mathrm{CDCl}_{3}\right) \delta 7.19(\mathrm{t}, J=9.0 \mathrm{~Hz}, 2 \mathrm{H}), 6.64(\mathrm{~d}, J=9.5 \mathrm{~Hz}, 2 \mathrm{H})$, $6.62(\mathrm{t}, J=9.0 \mathrm{~Hz}, 1 \mathrm{H}), 3.26(\mathrm{t}, J=8.0 \mathrm{~Hz}, 4 \mathrm{H}), 1.53-1.60(\mathrm{~m}, 4 \mathrm{H}), 1.35(\mathrm{sext}, J=7.5 \mathrm{~Hz}, 4 \mathrm{H}), 0.95$ $(\mathrm{t}, J=7.5 \mathrm{~Hz}, 6 \mathrm{H}) ;{ }^{13} \mathrm{C} \mathrm{NMR}\left(\mathrm{CDCl}_{3}\right) \delta 148.25,129.17,115.11,111.76,50.79,29.45,20.39,14.02$.<smiles>CCCCc1ccccc1</smiles>

Triphenylamine. ${ }^{19}$ (Table 7, entry 7). The general procedure A conducted with phenyl bromide $(0.157 \mathrm{~g}, 1.00 \mathrm{~mm} 0 \mathrm{l})$, diphenylamine $(0.203 \mathrm{~g}, 1.20 \mathrm{mmol}), \mathrm{Pd}(\mathrm{OAc})_{2}$ and CyPF- $t$-Bu Pd(OAc) $)_{2}(1.1$ $\left.\mathrm{mg}, 5.0 \times 10^{-3} \mathrm{mmol}\right)$ and CyPF- $t$-Bu $\left(2.8 \mathrm{mg}, 5.0 \times 10^{-3} \mathrm{mmol}\right)$ gave $211.3 \mathrm{mg}(86 \%)$ of triphenylamine as a white solid. ${ }^{1} \mathrm{H}$ NMR $\left(\mathrm{CDCl}_{3}\right) \delta 7.27(\mathrm{tt}, J=7.5,2.0 \mathrm{~Hz}, 6 \mathrm{H}), 7.12(\mathrm{dd}, J=8.5$, $1.0 \mathrm{~Hz}, 6 \mathrm{H}), 7.03(\mathrm{tt}, J=7.5,1.5 \mathrm{~Hz}, 3 \mathrm{H}), 3.35$ (s, $3 \mathrm{H}) ;{ }^{13} \mathrm{C} \mathrm{NMR}\left(\mathrm{CDCl}_{3}\right) \delta 147.82,129.18,124.13$, 122.63 .<smiles>Nc1ccccc1</smiles>

p-Tolylmorpholine. ${ }^{11}$ (Table 7, entry 8). The general procedure A conducted with $p$-tolyl bromide $(0.171 \mathrm{~g}, 1.00 \mathrm{~mm} 01)$, morpholine $(0.105 \mathrm{~g}, 1.20 \mathrm{mmol}), \mathrm{Pd}(\mathrm{OAc})_{2}\left(1.1 \mathrm{mg}, 5.0 \times 10^{-3} \mathrm{mmol}\right)$ and CyPF-t-Bu $\left(2.8 \mathrm{mg}, 5.0 \times 10^{-3} \mathrm{mmol}\right)$ gave $152.5 \mathrm{mg}(86 \%)$ of $p$-tolylmorpholine as a pale yellow liquid. ${ }^{1} \mathrm{H}$ NMR $\left(\mathrm{CDCl}_{3}\right) \delta 7.09(\mathrm{~d}, J=8.0 \mathrm{~Hz}, 2 \mathrm{H}), 6.83(\mathrm{~d}, J=8.5 \mathrm{~Hz}, 2 \mathrm{H}), 3.85(\mathrm{t}, J=5.0 \mathrm{~Hz}, 4 \mathrm{H})$, $3.10(\mathrm{t}, J=5.0 \mathrm{~Hz}, 4 \mathrm{H}), 2.27(\mathrm{~s}, 3 \mathrm{H}) ;{ }^{13} \mathrm{C} \mathrm{NMR}\left(\mathrm{CDCl}_{3}\right) \delta 149.16,129.68,129.53,115.99,66.92$, $49.84,20.36$.

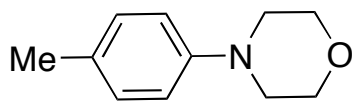

$N$-(3-Methoxyphenyl)morpholine. ${ }^{11}$ (Table 7, entry 9). The general procedure A conducted with 3bromoanisole $(0.187 \mathrm{~g}, 1.00 \mathrm{~mm} 01)$, morpholine $(0.105 \mathrm{~g}, 1.20 \mathrm{mmol}), \mathrm{Pd}(\mathrm{OAc})_{2}$ and CyPF- $t$-Bu $(50.0$ $\mu \mathrm{L}$ from stock solution $\left.\mathbf{A}, 5.0 \times 10^{-4} \mathrm{mmol}\right)$ gave $158.9 \mathrm{mg}(82 \%)$ of $\mathrm{N}$-(3-methoxyphenyl)morpholine as a pale yellow liquid. ${ }^{1} \mathrm{H} \mathrm{NMR}\left(\mathrm{CDCl}_{3}\right) \delta 7.18(\mathrm{t}, J=8.5 \mathrm{~Hz}, 1 \mathrm{H}), 6.53(\mathrm{ddd}, J=8.0,2.0,1.0 \mathrm{~Hz}, 1$ $\mathrm{H}), 6.42-6.45(\mathrm{~m}, 2 \mathrm{H}), 3.84(\mathrm{t}, J=5.0 \mathrm{~Hz}, 4 \mathrm{H}), 3.78(\mathrm{~s}, 3 \mathrm{H}), 3.14(\mathrm{t}, J=5.0 \mathrm{~Hz}, 4 \mathrm{H}) ;{ }^{13} \mathrm{C} \mathrm{NMR}$ $\left(\mathrm{CDCl}_{3}\right) \delta 160.00,152.67,129.83,108.43,104.68,102.18,66.86,55.15,49.25$. 


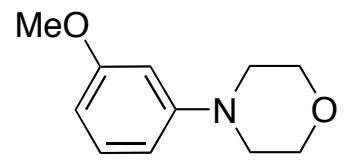

General Procedure C Using $\mathrm{LiN}\left(\mathrm{SiMe}_{3}\right)_{2}$ as the Base for Catalytic Amination of Functionalized Aryl Chlorides. The reaction conditions and average yields for each reaction are shown in Table 8-10. A typical procedure is given for the first entry in Table 8 .

2-Acetamido-5- $N$-benzylaminopyridine (Table 8, entry 1) 2-Acetamido-5-chloropyridine ( $0.168 \mathrm{~g}$, $1.00 \mathrm{mmol})$ was added to a $4 \mathrm{~mL}$ vial containing lithium bis(trimethylsilyl)amide $(0.402 \mathrm{~g}, 2.40 \mathrm{mmol})$ in $1.0 \mathrm{~mL}$ of DME. $\mathrm{Pd}(\mathrm{OAc})_{2}\left(1.1 \mathrm{mg}, 5.0 \times 10^{-3} \mathrm{mmol}\right)$, CyPF- $t$-Bu $\left(2.8 \mathrm{mg}, 5.0 \times 10^{-3} \mathrm{mmol}\right)$ were then added by syringe, followed by octylamine $(0.155 \mathrm{~g}, 1.20 \mathrm{mmol})$. The vial was sealed with a cap containing a PTFE septum, and the reaction mixture was stirred at $100^{\circ} \mathrm{C}$ for $24 \mathrm{~h}$. The reaction mixture then was allowed to cool to room temperature. To the reaction mixture was added $1 \mathrm{M} \mathrm{HCl}$ $(0.5-1.0 \mathrm{~mL})$. The mixture was stirred at room temperature for $5 \mathrm{~min}$ and was then extracted with $\mathrm{CH}_{2} \mathrm{Cl}_{2}(3 \times 30.0 \mathrm{~mL})$. The organic layer was separated and dried over $\mathrm{Na}_{2} \mathrm{SO}_{4}$. The solvent was evaporated, and the crude product was isolated by eluting with ethyl acetate to give $142.4 \mathrm{mg}$ (59\%) of 2-Acetamido-5- $N$-benzylaminopyridine as a white solid. ${ }^{1} \mathrm{H}$ NMR $\left(\mathrm{CDCl}_{3}\right) \delta 9.12(\mathrm{~s}, 1 \mathrm{H}), 7.94(\mathrm{~d}, J=$ $9.0 \mathrm{~Hz}, 1 \mathrm{H}), 7.60(\mathrm{~d}, J=2.5 \mathrm{~Hz}, 1 \mathrm{H}), 7.27(\mathrm{~d}, J=4.5 \mathrm{~Hz}, 4 \mathrm{H}), 7.20-7.22(\mathrm{~m}, 1 \mathrm{H}), 6.93(\mathrm{dd}, J=9.0$, $3.0 \mathrm{~Hz}, 1 \mathrm{H}), 4.25$ (s, $2 \mathrm{H}), 4.10$ (s, br, $1 \mathrm{H}), 1.98(\mathrm{~s}, 3 \mathrm{H}) ;{ }^{13} \mathrm{C} \mathrm{NMR}\left(\mathrm{CDCl}_{3}\right)$ d 168.27, 143.19, 141.09, 138.47, 131.36, 128.65, 127.37, 127.23, 122.50, 115.16, 48.18, 24.22. Anal. Calcd. For $\mathrm{C}_{14} \mathrm{H}_{15} \mathrm{~N}_{3} \mathrm{O}$ : C, 69.69; H, 6.27; N, 17.41. Found: C, 69.59; H, 6.33; N, 17.20.<smiles>CC(=O)Nc1ccc(NC(=O)c2ccccc2)cn1</smiles>

2-Acetamido-5- $N$-sec-butylaminopyridine (Table 8, entry 2) The general procedure C conducted with 2-acetamido-5-chloropyridine $(0.168 \mathrm{~g}, 1.00 \mathrm{mmol})$, sec-butylamine $(0.119 \mathrm{~g}, 1.20 \mathrm{mmol})$, $\mathrm{Pd}(\mathrm{OAc})_{2}\left(1.1 \mathrm{mg}, 5.0 \times 10^{-3} \mathrm{mmol}\right), \quad \mathrm{CyPF}-t-\mathrm{Bu}\left(2.8 \mathrm{mg}, 5.0 \times 10^{-3} \mathrm{mmol}\right)$ and lithium bis(trimethylsilyl)amide $(0.402 \mathrm{~g}, 2.40 \mathrm{mmol})$ gave $198.3 \mathrm{mg}(96 \%)$ of 2-Acetamido-5- $\mathrm{N}$-secbutylaminopyridine as a white solid. ${ }^{1} \mathrm{H}$ NMR $\left(\mathrm{CDCl}_{3}\right) \delta 9.35(\mathrm{~s}, 1 \mathrm{H}), 7.95(\mathrm{~d}, J=9.0 \mathrm{~Hz}, 1 \mathrm{H}), 7.60$ $(\mathrm{d}, J=2.5 \mathrm{~Hz}, 1 \mathrm{H}), 6.91$ (dd, $J=9.0,2.5 \mathrm{~Hz}, 1 \mathrm{H}), 3.40$ (s, br, $1 \mathrm{H}$ ), 3.30 (sext, $J=6.5 \mathrm{~Hz}, 1 \mathrm{H}$ ), 2.09 $(\mathrm{s}, 3 \mathrm{H}), 1.50-1.56(\mathrm{~m}, 1 \mathrm{H}), 1.39-1.44(\mathrm{~m}, 1 \mathrm{H}), 1.11(\mathrm{~d}, J=6.5 \mathrm{~Hz}, 3 \mathrm{H}), 0.89(\mathrm{t}, J=7.0 \mathrm{~Hz}, 3 \mathrm{H}) ;{ }^{13} \mathrm{C}$ NMR $\left(\mathrm{CDCl}_{3}\right) \delta 168.22,142.62,140.83,132.14,122.53,115.36,50.05,29.32,24.19,19.91,10.15$.<smiles>CCC(C)Nc1ccc(NC(C)=O)nc1</smiles>

5-Acetamido-2- $N$-benzylaminopyridine (Table 8, entry 3) The general procedure $\mathrm{C}$ conducted with 5-acetamido-2-chloropyridine $(0.168 \mathrm{~g}, 1.00 \mathrm{mmol})$, benzylamine $(0.129 \mathrm{~g}, 1.20 \mathrm{mmol}), \mathrm{Pd}(\mathrm{OAc})_{2}(1.1$ $\left.\mathrm{mg}, 5.0 \times 10^{-3} \mathrm{mmol}\right)$, CyPF-t-Bu $\left(2.8 \mathrm{mg}, 5.0 \times 10^{-3} \mathrm{mmol}\right)$ and lithium bis(trimethylsilyl)amide $(0.402 \mathrm{~g}, 2.40 \mathrm{mmol})$ gave $175.5 \mathrm{mg}(73 \%)$ of 2-Acetamido-5- $N$-benzylaminopyridine as a white solid. ${ }^{1} \mathrm{H}$ NMR $\left(\mathrm{CD}_{3} \mathrm{OD}\right) \delta 8.05(\mathrm{dd}, J=2.5,1.0 \mathrm{~Hz}, 1 \mathrm{H}), 7.59(\mathrm{dd}, J=9.0,3.0 \mathrm{~Hz}, 1 \mathrm{H}), 7.32(\mathrm{~d}, J=7.5$ $\mathrm{Hz}, 2 \mathrm{H}), 7.28(\mathrm{td}, J=7.0,1.5 \mathrm{~Hz}, 2 \mathrm{H}), 7.20(\mathrm{t}, J=7.5 \mathrm{~Hz}, 1 \mathrm{H}), 6.50(\mathrm{dd}, J=9.0,1.0 \mathrm{~Hz}, 1 \mathrm{H}), 4.46$ 
(s, $2 \mathrm{H}), 2.07(\mathrm{~s}, 3 \mathrm{H}) ;{ }^{13} \mathrm{C}$ NMR $\left(\mathrm{CD}_{3} \mathrm{OD}\right) \delta$ 171.70, 157.55, 141.10, 140.92, 133.18, 129.42, $128.32,127.90,126.57,109.24,46.54,23.26$.<smiles>CC(=O)Nc1ccc(NBr)nc1</smiles>

5-Acetamido-2- $\mathrm{N}$-iso-butylaminopyridine (Table 8, entry 4) The general procedure C conducted with 5-acetamido-2-chloropyridine $(0.168 \mathrm{~g}, 1.00 \mathrm{mmol})$, iso-butylamine $(0.119 \mathrm{~g}, 1.20 \mathrm{mmol})$, $\mathrm{Pd}(\mathrm{OAc})_{2}\left(1.1 \mathrm{mg}, 5.0 \times 10^{-3} \mathrm{mmol}\right)$, CyPF- $t$-Bu $\left(2.8 \mathrm{mg}, 5.0 \times 10^{-3} \mathrm{mmol}\right)$ and lithium bis(trimethylsilyl)amide $(0.402 \mathrm{~g}, 2.40 \mathrm{mmol})$ gave $121.1 \mathrm{mg}(67 \%)$ of 5-Acetamido-2- $N$-isobutylaminopyridine as a white solid. ${ }^{1} \mathrm{H} \mathrm{NMR}\left(\mathrm{CD}_{3} \mathrm{OD}\right) \delta 7.60(\mathrm{~d}, J=9.0 \mathrm{~Hz}, 1 \mathrm{H}), 7.58(\mathrm{~s}, 1 \mathrm{H}), 6.91$ $(\mathrm{dd}, J=9.0,3.0 \mathrm{~Hz}, 1 \mathrm{H}), 2.78(\mathrm{~d}, J=8.5 \mathrm{~Hz}, 2 \mathrm{H}), 2.02(\mathrm{~s}, 3 \mathrm{H}), 1.72-1.79(\mathrm{~m}, 1 \mathrm{H}), 0.87(\mathrm{~d}, J=6.5$ $\mathrm{Hz}, 6 \mathrm{H}) ;{ }^{13} \mathrm{C}$ NMR $\left(\mathrm{CD}_{3} \mathrm{OD}\right) \delta 171.24,144.42,142.28,132.98,122.08,117.44,52.49,29.02,23.58$, 20.76 .

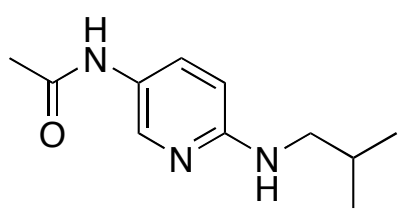

2-Hydroxy-5- $N$-octylaminopyridine (Table 8, entry 5) The general procedure $\mathrm{C}$ conducted with 5chloro-2-hydroxypyridine $(0.130 \mathrm{~g}, 1.00 \mathrm{mmol})$, octylamine $(0.155 \mathrm{~g}, 1.20 \mathrm{mmol}), \mathrm{Pd}(\mathrm{OAc})_{2}(1.1 \mathrm{mg}$, $\left.5.0 \times 10^{-3} \mathrm{mmol}\right)$, CyPF- $t$-Bu $\left(2.8 \mathrm{mg}, 5.0 \times 10^{-3} \mathrm{mmol}\right)$ and lithium bis(trimethylsilyl)amide $(0.402 \mathrm{~g}$, $2.40 \mathrm{mmol})$ gave $117.6 \mathrm{mg}(53 \%)$ of 2 -Hydroxy-5-N-octylaminopyridine as a white solid. ${ }^{1} \mathrm{H}$ NMR $\left(\mathrm{CDCl}_{3}\right) \delta 13.63$ (s. br, $\left.1 \mathrm{H}\right), 7.07(\mathrm{dd}, J=9.5,2.5 \mathrm{~Hz}, 1 \mathrm{H}), 6.62(\mathrm{~d}, J=2.5 \mathrm{~Hz}, 1 \mathrm{H}), 6.48(\mathrm{~d}, J=10.0$ $\mathrm{Hz}, 1 \mathrm{H}$ ), 3.20 (s, br, $1 \mathrm{H}$ ), 2.83 (t, $J=7.0 \mathrm{~Hz}, 2 \mathrm{H}), 1.53$ (quint, $J=7.0 \mathrm{~Hz}, 2 \mathrm{H}), 1.23-1.32(\mathrm{~m}, 10 \mathrm{H}$ ), $0.84(\mathrm{~d}, J=67.0 \mathrm{~Hz}, 3 \mathrm{H}) ;{ }^{13} \mathrm{C}$ NMR $\left(\mathrm{CDCl}_{3}\right) \delta 162.35,135.37,131.93,120.31,114.20,45.06,31.72$, 29.29, 29.19, 29.15, 27.07, 22.57, 14.02. Anal. Calcd. For $\mathrm{C}_{13} \mathrm{H}_{22} \mathrm{~N}_{2} \mathrm{O}$ : C, 70.23; H, 9.97; N, 12.60. Found: C, 69.96; H, 10.01; N, 12.42 .<smiles>Oc1ccc(Nc2ccccc2)cn1</smiles>

3-Hydroxy-5- $N$-cyclohexylaminopyridine (Table 8, entry 6) The general procedure C conducted with 5-chloro-3-hydroxypyridine $(0.130 \mathrm{~g}, 1.00 \mathrm{mmol})$, cyclohexylamine $(0.119 \mathrm{~g}, 1.20 \mathrm{mmol})$, $\mathrm{Pd}(\mathrm{OAc})_{2}\left(1.1 \mathrm{mg}, 5.0 \times 10^{-3} \mathrm{mmol}\right)$, CyPF- $t$-Bu $\left(2.8 \mathrm{mg}, 5.0 \times 10^{-3} \mathrm{mmol}\right)$ and lithium bis(trimethylsilyl)amide $(0.402 \mathrm{~g}, 2.40 \mathrm{mmol})$ gave $110.6 \mathrm{mg}(67 \%)$ of 3-Hydroxy-5- $\mathrm{N}$ cyclohexylaminopyridine as a white solid. ${ }^{1} \mathrm{H}$ NMR $\left(\mathrm{CD}_{3} \mathrm{OD}\right) \delta 7.40(\mathrm{~d}, J=2.5 \mathrm{~Hz}, 1 \mathrm{H}), 7.30(\mathrm{~d}, J=$ $2.5 \mathrm{~Hz}, 1 \mathrm{H}), 6.43(\mathrm{t}, J=2.5 \mathrm{~Hz}, 1 \mathrm{H}), 3.15(\mathrm{tt}, J=10.0,4.0 \mathrm{~Hz}, 1 \mathrm{H}), 1.98(\mathrm{dd}, J=12.5,3.0 \mathrm{~Hz}, 2 \mathrm{H})$, $1.76(\mathrm{dt}, J=13.5,3.5 \mathrm{~Hz}, 2 \mathrm{H}), 1.65(\mathrm{dt}, J=12.5,3.5 \mathrm{~Hz}, 1 \mathrm{H}), 1.33-1.42(\mathrm{~m}, 2 \mathrm{H}), 1.14-1.27(\mathrm{~m}, 3$ $\mathrm{H}) ;{ }^{13} \mathrm{C}$ NMR $\left(\mathrm{CD}_{3} \mathrm{OD}\right) \delta 156.57,147.27,127.85,125.42,106.94,52.42,33.92,27.00,26.09$.<smiles>Oc1cncc(NCl)c1</smiles> 
5-Hydroxymethyl-2- $N$-octylaminopyridine (Table 8, entry 7 ) The general procedure $\mathrm{C}$ conducted with 2-chloro-5-hydroxymethylpyridine $(0.144 \mathrm{~g}, 1.00 \mathrm{mmol})$, octylamine $(0.155 \mathrm{~g}, 1.20$ $\mathrm{mmol}), \mathrm{Pd}(\mathrm{OAc})_{2}\left(1.1 \mathrm{mg}, 5.0 \times 10^{-3} \mathrm{mmol}\right)$, CyPF- $t$-Bu $\left(2.8 \mathrm{mg}, 5.0 \times 10^{-3} \mathrm{mmol}\right)$ and lithium bis(trimethylsilyl)amide $(0.402 \mathrm{~g}, 2.40 \mathrm{mmol})$ gave $187.0 \mathrm{mg}$ (79\%) of 5-Hydroxymethyl-2- $\mathrm{N}$ octylaminopyridine as a white solid. ${ }^{1} \mathrm{H}$ NMR $\left(\mathrm{CDCl}_{3}\right) \delta 7.85(\mathrm{~d}, J=2.0 \mathrm{~Hz}, 1 \mathrm{H}), 7.42(\mathrm{dd}, J=8.5$, $2.0 \mathrm{~Hz}, 1 \mathrm{H}), 6.32(\mathrm{~d}, J=8.5 \mathrm{~Hz}, 1 \mathrm{H}), 4.59(\mathrm{~s}, 1 \mathrm{H}), 4.44(\mathrm{~s}, 2 \mathrm{H}), 3.35$ (s, br, $1 \mathrm{H}), 3.15$ (td, $J=7.0$, $5.0 \mathrm{~Hz}, 2 \mathrm{H}$ ), 1.55 (quint, $J=7.5 \mathrm{~Hz}, 2 \mathrm{H}), 1.23-1.35(\mathrm{~m}, 10 \mathrm{H}), 0.84(\mathrm{t}, J=7.0 \mathrm{~Hz}, 3 \mathrm{H}) ;{ }^{13} \mathrm{C} \mathrm{NMR}$ $\left(\mathrm{CDCl}_{3}\right) \delta 158.45,147.28,137.78,125.07,106.10,62.47,42.37,31.76,29.43,29.31,29.19,27.00$, 22.59, 14.04. Anal. Calcd. For $\mathrm{C}_{14} \mathrm{H}_{24} \mathrm{~N}_{2} \mathrm{O}: \mathrm{C}, 71.14 ; \mathrm{H}, 10.23 ; \mathrm{N}, 11.85$. Found: $\mathrm{C}, 71.39 ; \mathrm{H}, 10.26 ; \mathrm{N}$, 11.46 .

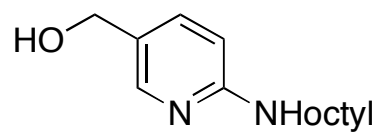

5-Hydroxymethyl-2- $N$-benzylaminopyridine (Table 8, entry 8) The general procedure $C$ conducted with 2-chloro-5-hydroxymethylpyridine $(0.144 \mathrm{~g}, 1.00 \mathrm{mmol})$, benzylamine $(0.129 \mathrm{~g}, 1.20 \mathrm{mmol})$, $\mathrm{Pd}(\mathrm{OAc})_{2}\left(1.1 \mathrm{mg}, 5.0 \times 10^{-3} \mathrm{mmol}\right)$, CyPF- $t$-Bu $\left(2.8 \mathrm{mg}, 5.0 \times 10^{-3} \mathrm{mmol}\right)$ and lithium bis(trimethylsilyl)amide $(0.402 \mathrm{~g}, 2.40 \mathrm{mmol})$ gave $117.9 \mathrm{mg}(55 \%)$ of 5-Hydroxymethyl-2- $\mathrm{N}$ benzylaminopyridine as a white solid. ${ }^{1} \mathrm{H}$ NMR $\left(\mathrm{CD}_{3} \mathrm{OD}\right) \delta 7.89(\mathrm{~d}, J=2.0 \mathrm{~Hz}, 1 \mathrm{H}), 7.43(\mathrm{dd}, J=9.0$, $2.0 \mathrm{~Hz}, 1 \mathrm{H}), 7.32(\mathrm{~d}, J=7.0 \mathrm{~Hz}, 2 \mathrm{H}), 7.27(\mathrm{t}, J=7.0 \mathrm{~Hz}, 2 \mathrm{H}), 7.19(\mathrm{t}, J=7.0 \mathrm{~Hz}, 1 \mathrm{H}), 6.52(\mathrm{~d}, J=$ $9.0 \mathrm{~Hz}, 1 \mathrm{H}), 4.89$ (s, $2 \mathrm{H}), 4.47$ (s, $2 \mathrm{H}), 4.41(\mathrm{~s}, 2 \mathrm{H}) ;{ }^{13} \mathrm{C} \mathrm{NMR}\left(\mathrm{CD}_{3} \mathrm{OD}\right) \delta$ 159.82, 147.27, 140.99, 138.96, 129.40, 128.28, 127.89, 126.32, 109.54, 62.74, 46.38. Anal. Calcd. For $\mathrm{C}_{13} \mathrm{H}_{14} \mathrm{~N}_{2} \mathrm{O}: \mathrm{C}, 72.87$; H, 6.59; N, 13.07. Found: C, 72.60; H, 6.58; N, 12.90 .

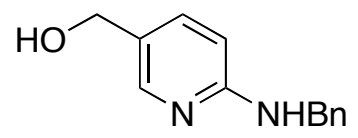

General Procedure D Using $\mathrm{K}_{3} \mathrm{PO}_{4}$ as the Base for Catalytic Amination of Functionalized Aryl Iodides. The reaction conditions and average yields for each reaction are shown in Table 9. A typical procedure is given Table 6 , entry 5 .

4- $\mathrm{N}$-Octylamino-benzoic acid. (Table 9, entry 1) 4-Chlorobenzoic acid ( $0.157 \mathrm{~g}, 1.00 \mathrm{mmol})$ was added to a $4 \mathrm{~mL}$ vial containing lithium bis(trimethylsilyl)amide $(0.402 \mathrm{~g}, 2.40 \mathrm{mmol})$ in $1.0 \mathrm{~mL}$ of DME. $\mathrm{Pd}(\mathrm{OAc})_{2}$ and CyPF- $t$-Bu $\left(50.0 \mu \mathrm{L}\right.$ from stock solution $\left.\mathbf{A}, 5.0 \times 10^{-4} \mathrm{mmol}\right)$ were then added by syringe, followed by octylamine $(0.155 \mathrm{~g}, 1.20 \mathrm{mmol})$. The vial was sealed with a cap containing a PTFE septum, and the reaction mixture was stirred at $100^{\circ} \mathrm{C}$ for $20 \mathrm{~h}$. The reaction mixture then was allowed to cool to room temperature. To the reaction mixture was added $1 \mathrm{M} \mathrm{HCl}(0.5-1.0 \mathrm{~mL})$. The mixture was stirred at room temperature for $5 \mathrm{~min}$ and was then extracted with $\mathrm{CH}_{2} \mathrm{Cl}_{2}(3 \times 30.0 \mathrm{~mL})$. The organic layer was separated and dried over $\mathrm{Na}_{2} \mathrm{SO}_{4}$. The solvent was evaporated, and the crude product was isolated by eluting with hexane/ethyl acetate (50/50) to give $202.2 \mathrm{mg}(81 \%)$ of $\mathrm{N}$-octylaminobenzoic acid as a white solid. ${ }^{1} \mathrm{H}$ NMR $\left(\mathrm{CD}_{3} \mathrm{OD}\right) \delta 7.75(\mathrm{~d}, J=9.2 \mathrm{~Hz}, 2 \mathrm{H}), 6.55(\mathrm{~d}, J=9.2 \mathrm{~Hz}$, $2 \mathrm{H}$ ), 4.70-5.20 (s, b, $2 \mathrm{H}$ ), 3.09 (t, $J=7.2 \mathrm{~Hz}, 2 \mathrm{H}$ ), 1.59 (quint, $J=7.2 \mathrm{~Hz}, 2 \mathrm{H}$ ), 1.27-1.35 (m, $10 \mathrm{H}$ ), $0.88(\mathrm{t}, J=7.0 \mathrm{~Hz}, 3 \mathrm{H}) ;{ }^{13} \mathrm{C}$ NMR $\left(\mathrm{CD}_{3} \mathrm{OD}\right) \delta 171.02,154.82,132.92,117.93,112.09,44.15,33.17$, 30.72, 30.60, 30.31, 28.40, 23.89, 14.62. Anal. Calcd. For: $\mathrm{C}_{15} \mathrm{H}_{23} \mathrm{NO}_{2} \mathrm{C}, 72.25 ; \mathrm{H}, 9.30 ; \mathrm{N}, 5.62$. Found: C, 72.19; H, 9.43; N, 5.56. 


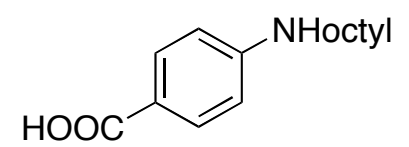

4-N-Octylamino-benzoic acid. (Table 9, entry 2) The general procedure C conducted with 4bromobenzoic acid $(0.201 \mathrm{~g}, 1.00 \mathrm{mmol})$, octylamine $(0.155 \mathrm{~g}, 1.20 \mathrm{mmol}), \mathrm{Pd}(\mathrm{OAc})_{2}$ and $\mathrm{CyPF}-t$-Bu $\left(50.0 \mu \mathrm{L}\right.$ from stock solution $\left.\mathbf{A}, 5.0 \times 10^{-4} \mathrm{mmol}\right)$ gave $179.4 \mathrm{mg}(72 \%)$ of $N$-octyl-aminobenzoic acid as a white solid. $\left({ }^{1} \mathrm{H}\right.$ and ${ }^{13} \mathrm{C}$ NMR spectra are the same as those for the product in Table 9, entry 1 .)<smiles>O=C(O)c1ccc(NOC(=O)c2ccccc2)cc1</smiles>

4- $\mathrm{N}$-sec-butylamino-benzoic acid (Table 9, entry 3) The general procedure C conducted with 4bromobenzoic acid $(0.201 \mathrm{~g}, 1.00 \mathrm{mmol})$, sec-butylamine $(87.8 \mathrm{mg}, 1.20 \mathrm{mmol}), \mathrm{Pd}(\mathrm{OAc})_{2}$ and CyPF$t$-Bu $\left(50.0 \mu \mathrm{L}\right.$ from stock solution $\left.\mathbf{A}, 5.0 \times 10^{-4} \mathrm{mmol}\right)$ and lithium bis(trimethylsilyl)amide $(0.402 \mathrm{~g}$, $2.40 \mathrm{mmol})$ gave $178.4 \mathrm{mg}(92 \%)$ of $4-N$-sec-butylbenzoic acid as a white solid. ${ }^{1} \mathrm{H} \mathrm{NMR}\left(\mathrm{CDCl}_{3}\right) \delta$ 7.25-9.00 (s, b, $1 \mathrm{H}), 7.89(\mathrm{~d}, J=6.8 \mathrm{~Hz}, 2 \mathrm{H}), 6.52(\mathrm{~d}, J=6.8 \mathrm{~Hz}, 2 \mathrm{H}), 4.70-5.20(\mathrm{~s}, \mathrm{~b}, 1 \mathrm{H}), 3.47$ (sext, $J=5.2 \mathrm{~Hz}, 1 \mathrm{H}), 1.46-1.62(\mathrm{~m}, 2 \mathrm{H}), 1.19(\mathrm{~d}, J=4.8 \mathrm{~Hz}, 3 \mathrm{H}), 0.94(\mathrm{t}, J=6.0 \mathrm{~Hz}, 3 \mathrm{H}) ;{ }^{13} \mathrm{C}$ $\operatorname{NMR}\left(\mathrm{CDCl}_{3}\right) \delta 172.55,152.03,132.37,116.67,111.61,49.53,29.51,20.04,10.29$. Anal. Calcd. For $\mathrm{C}_{11} \mathrm{H}_{15} \mathrm{NO}_{2}$ : C, 68.37; H, 7.82; N, 7.25. Found: C, 68.35; H, 7.80; N, 7.17.

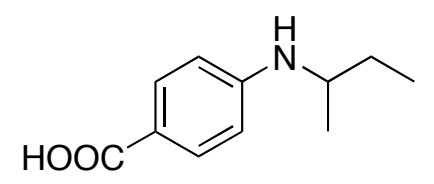

4-N-sec-butylamino-benzoic acid (Table 9, entry 4) The general procedure C conducted with 4iodobenzoic acid $(0.248 \mathrm{~g}, 1.00 \mathrm{mmol})$, sec-butylamine $(87.8 \mathrm{mg}, 1.20 \mathrm{mmol}), \mathrm{Pd}(\mathrm{OAc})_{2}$ and CyPF-t$\mathrm{Bu}\left(50.0 \mu \mathrm{L}\right.$ from stock solution $\left.\mathbf{A}, 5.0 \times 10^{-4} \mathrm{mmol}\right)$ and lithium bis(trimethylsilyl)amide $(0.402 \mathrm{~g}$, $2.40 \mathrm{mmol})$ gave $151.3 \mathrm{mg}(92 \%)$ of $4-\mathrm{N}$-sec-butylbenzoic acid as a white solid. $\left({ }^{1} \mathrm{H}\right.$ and ${ }^{13} \mathrm{C}$ NMR spectra are the same as those for the product in Table 9, entry 3.)<smiles>CCC(C)Nc1ccc(C(=O)O)cc1</smiles>

4-N-Iso-Butylamino-benzamide (Table 9, entry 5) The general procedure C conducted with 4chlorobenzamide $(0.156 \mathrm{~g}, 1.00 \mathrm{mmol})$, iso-butylamine $(87.8 \mathrm{mg}, 1.20 \mathrm{mmol}), \mathrm{Pd}(\mathrm{OAc})_{2}(1.1 \mathrm{mg}, 5.0$ $\left.\times 10^{-3} \mathrm{mmol}\right)$, CyPF-t-Bu $\left(2.8 \mathrm{mg}, 5.0 \times 10^{-3} \mathrm{mmol}\right)$ and lithium bis(trimethylsilyl)amide $(0.402 \mathrm{~g}$, $2.40 \mathrm{mmol})$ gave $129.6 \mathrm{mg}(67 \%)$ of $4-N$-iso-butylamino-benzamide as a white solid. ${ }^{1} \mathrm{H}$ NMR $\left(\mathrm{CDCl}_{3}\right) \delta 7.63(\mathrm{~d}, J=7.2 \mathrm{~Hz}, 2 \mathrm{H}), 6.53(\mathrm{~d}, J=7.2 \mathrm{~Hz}, 2 \mathrm{H}), 5.96(\mathrm{~s}, \mathrm{~b}, 2 \mathrm{H}), 4.04$ (s, b, $\left.1 \mathrm{H}\right), 2.94(\mathrm{~d}$, $J=5.2 \mathrm{~Hz}, 2 \mathrm{H}), 1.86$ (nonet, $J=5.2 \mathrm{~Hz}, 1 \mathrm{H}), 0.95(\mathrm{~d}, J=5.6 \mathrm{~Hz}, 6 \mathrm{H}) ;{ }^{13} \mathrm{C} \mathrm{NMR}\left(\mathrm{CDCl}_{3}\right) \delta 169.50$, 151.52, 129.23, 120.87, 111.48, 51.13, 27.98, 20.33. Anal. Calcd. For $\mathrm{C}_{11} \mathrm{H}_{16} \mathrm{~N}_{2} \mathrm{O}$ : C, 68.72; $\mathrm{H}, 8.39$; N, 14.57. Found: C, 68.70; H, 8.43; N, 14.32.<smiles>CC(C)CNc1ccc(C(N)=O)cc1</smiles> 
4-N-Iso-Butylamino-benzamide (Table 9, entry 6) The general procedure B conducted with 4bromobenzamide $(0.200 \mathrm{~g}, 1.00 \mathrm{mmol})$, iso-butylamine $(87.8 \mathrm{mg}, 1.20 \mathrm{mmol}), \mathrm{Pd}(\mathrm{OAc})_{2}(1.1 \mathrm{mg}, 5.0$ $\left.\times 10^{-3} \mathrm{mmol}\right)$, CyPF-t-Bu $\left(2.8 \mathrm{mg}, 5.0 \times 10^{-3} \mathrm{mmol}\right)$ and lithium bis(trimethylsilyl)amide $(0.402 \mathrm{~g}$, $2.40 \mathrm{mmol})$ gave $162.5 \mathrm{mg}(85 \%)$ of $4-\mathrm{N}$-Iso-Butylamino-benzamide as a white solid. $\left({ }^{1} \mathrm{H}\right.$ and ${ }^{13} \mathrm{C}$ NMR spectra are the same as those for the product in Table 9, entry 6.)<smiles>CC(C)CNc1ccc(C(N)=O)cc1</smiles>

4-N-octylamino-acetophenone (Table 9, entry 7) The general procedure B conducted with 4'-chloroacetophenone $(0.155 \mathrm{~g}, 1.00 \mathrm{mmol})$, octylamine $(0.155 \mathrm{~g}, 1.20 \mathrm{mmol}), \mathrm{Pd}(\mathrm{OAc})_{2}\left(1.1 \mathrm{mg}, 5.0 \times 10^{-3}\right.$ $\mathrm{mmol})$, CyPF-t-Bu $\left(2.8 \mathrm{mg}, 5.0 \times 10^{-3} \mathrm{mmol}\right)$ and lithium bis(trimethylsilyl)amide $(0.402 \mathrm{~g}, 2.40$ mmol) in DME $(5.0 \mathrm{~mL})$ gave $225.6 \mathrm{mg}(91 \%)$ of 4 '-N-octylamino-acetophenone as a pale yellow liquid. ${ }^{1} \mathrm{H}$ NMR $\left(\mathrm{CDCl}_{3}\right) \delta 7.78(\mathrm{~d}, J=8.8 \mathrm{~Hz}, 2 \mathrm{H}), 6.51(\mathrm{~d}, J=8.8 \mathrm{~Hz}, 2 \mathrm{H}), 4.1(\mathrm{~s}, \mathrm{~b}, 1 \mathrm{H}), 3.13(\mathrm{q}, J$ $=6.4 \mathrm{~Hz}, 2 \mathrm{H}), 2.46(\mathrm{~s}, 3 \mathrm{H}), 1.60$ (quint, $J=7.2 \mathrm{~Hz}, 2 \mathrm{H}), 1.25-1.36(\mathrm{~m}, 10 \mathrm{H}), 0.86(\mathrm{t}, J=6.8 \mathrm{~Hz}, 3$ $\mathrm{H}) ;{ }^{13} \mathrm{C} \mathrm{NMR}\left(\mathrm{CDCl}_{3}\right) \delta 196.13,152.34,130.72,126.25,111.13,43.23,31.70,29.25,29.21,29.13$, 26.98, 25.84, 22.55, 13.98. Anal. Calcd. For $\mathrm{C}_{16} \mathrm{H}_{25} \mathrm{NO}$ : C, 77.68; H, 10.19; N, 5.66. Found: C, 77.59; H, 10.32; N, 5.58 .

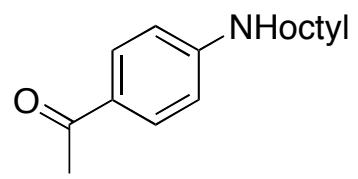

4'-N-octylamino-acetophenone (Table 9, entry 8). The general procedure D conducted with 4'chloro-acetophenone $(0.155 \mathrm{~g}, 1.00 \mathrm{mmol})$, octylamine $(0.155 \mathrm{~g}, 1.20 \mathrm{mmol}), \mathrm{Pd}(\mathrm{OAc})_{2}(1.1 \mathrm{mg}, 5.0 \times$ $\left.10^{-3} \mathrm{mmol}\right)$, CyPF- $t$-Bu $\left(2.8 \mathrm{mg}, 5.0 \times 10^{-3} \mathrm{mmol}\right)$ and potassium phosphate $(0.254 \mathrm{~g}, 1.40 \mathrm{mmol})$ gave $178.5 \mathrm{mg}(74 \%)$ of 4 '- $N$-octylamino-acetophenone as a pale yellow liquid. $\left({ }^{1} \mathrm{H}\right.$ and ${ }^{13} \mathrm{C}$ NMR spectra are the same as those for the product in Table 9, entry 7.)<smiles>CC(=O)Nc1ccc(C(C)=O)cc1</smiles>

4-N-octylamino-acetophenone (Table 9, entry 9) The general procedure C conducted with 4'-chloroacetophenone $(0.155 \mathrm{~g}, 1.00 \mathrm{mmol})$, octylamine $(0.155 \mathrm{~g}, 1.20 \mathrm{mmol}), \mathrm{Pd}(\mathrm{OAc})_{2}$ and CyPF-t-Bu $(50.0$ $\mu \mathrm{L}$ from stock solution $\left.\mathbf{A}, 5.0 \times 10^{-4} \mathrm{mmol}\right)$ and lithium bis(trimethylsilyl)amide $(0.402 \mathrm{~g}, 2.40 \mathrm{mmol})$ gave $151.2 \mathrm{mg}(61 \%)$ of 4 '- $N$-octylamino-acetophenone as a pale yellow liquid. $\left({ }^{1} \mathrm{H}\right.$ and ${ }^{13} \mathrm{C}$ NMR spectra are the same as those for the product in Table 9, entry 7.)<smiles>CC(=O)c1ccc(NOc2ccccc2)cc1</smiles> 
4'-N-octylamino-acetophenone (Table 9, entry 10). The general procedure D conducted with 4'iodoacetophenone $(0.246 \mathrm{~g}, 1.00 \mathrm{mmol})$, octylamine $(0.155 \mathrm{~g}, 1.20 \mathrm{mmol}), \mathrm{Pd}(\mathrm{OAc})_{2}(1.1 \mathrm{mg}, 5.0 \times$ $\left.10^{-3} \mathrm{mmol}\right)$, CyPF- $t$-Bu $\left(2.8 \mathrm{mg}, 5.0 \times 10^{-3} \mathrm{mmol}\right)$ and potassium phosphate $(0.254 \mathrm{~g}, 1.40 \mathrm{mmol})$ gave $192.9 \mathrm{mg}(78 \%)$ of 4 '- $N$-octylamino-acetophenone as a pale yellow liquid. $\left({ }^{1} \mathrm{H}\right.$ and ${ }^{13} \mathrm{C}$ NMR spectra are the same as those for the product in Table 9, entry 7.)<smiles>CC(=O)c1ccc(NOc2ccccc2)cc1</smiles>

4- $N$-cyclohexylamino-acetophenone (Table 9, entry 11) The general procedure C conducted with 4'chloro-acetophenone $(0.155 \mathrm{~g}, 1.00 \mathrm{mmol})$, cyclohexylamine $(0.119 \mathrm{~g}, 1.20 \mathrm{mmol}), \mathrm{Pd}(\mathrm{OAc})_{2}(1.1 \mathrm{mg}$, $\left.5.0 \times 10^{-3} \mathrm{mmol}\right)$, CyPF-t-Bu $\left(2.8 \mathrm{mg}, 5.0 \times 10^{-3} \mathrm{mmol}\right)$ and lithium bis(trimethylsilyl)amide $(0.402 \mathrm{~g}$, $2.40 \mathrm{mmol})$ gave $151.6 \mathrm{mg}(87 \%)$ of $4-N$-cyclohexylamino-acetophenone as a pale yellow liquid. ${ }^{1} \mathrm{H}$ NMR $\left(\mathrm{CDCl}_{3}\right) \delta 7.76(\mathrm{dt}, J=9.0,2.0 \mathrm{~Hz}, 2 \mathrm{H}), 6.49(\mathrm{dt}, J=9.0,2.0 \mathrm{~Hz}, 2 \mathrm{H}), 4.27(\mathrm{~s}, \mathrm{~b}, 1 \mathrm{H}), 3.28$ $3.32(\mathrm{~m}, 1 \mathrm{H}), 2.44(\mathrm{~s}, 3 \mathrm{H}), 2.00(\mathrm{dd}, J=12.5,3.0 \mathrm{~Hz}, 2 \mathrm{H}), 1.73(\mathrm{dt}, J=13.0,4.0 \mathrm{~Hz}, 2 \mathrm{H}), 1.62(\mathrm{dt}, J$ $=13.0,4.0 \mathrm{~Hz}, 1 \mathrm{H}), 1.30-1.39(\mathrm{~m}, 2 \mathrm{H}), 1.12-1.23(\mathrm{~m}, 3 \mathrm{H}) ;{ }^{13} \mathrm{C} \mathrm{NMR}\left(\mathrm{CDCl}_{3}\right) \delta 196.09,151.29$, 130.75, 125.88, 111.36, 51.09, 32.92, 25.79, 25.57, 24.70. Anal. Calcd. For: $\mathrm{C}_{14} \mathrm{H}_{19} \mathrm{NO} \mathrm{C}, 77.38$; H, 8.81 ; N, 6.45. Found: C, 77.44; H, 8.95; N, 6.55 .

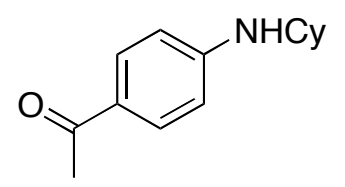

4'-N-octylamino-acetophenone (Table 9, entry 12). The general procedure D conducted with 4'bromo-acetophenone $(0.199 \mathrm{~g}, 1.00 \mathrm{mmol})$, cyclohexylamine $(0.119 \mathrm{~g}, 1.20 \mathrm{mmol}), \mathrm{Pd}(\mathrm{OAc})_{2}(2.2 \mathrm{mg}$, $\left.1.0 \times 10^{-2} \mathrm{mmol}\right)$, CyPF-t-Bu $\left(5.5 \mathrm{mg}, 1.0 \times 10^{-2} \mathrm{mmol}\right)$ and potassium phosphate $(0.254 \mathrm{~g}, 1.40$ mmol) gave $142.9 \mathrm{mg}(82 \%)$ of $4{ }^{\prime}-N$-octylamino-acetophenone as a pale yellow liquid. $\left({ }^{1} \mathrm{H}\right.$ and ${ }^{13} \mathrm{C}$ NMR spectra are the same as those for the product in Table 9, entry 11.)<smiles>CC(=O)c1ccc(NCl)cc1</smiles>

4-N-Octylamino-acetanilide (Table 9, entry 13) The general procedure $\mathrm{C}$ conducted with 4bromoacetanilide $(0.214 \mathrm{~g}, 1.00 \mathrm{mmol})$, octylamine $(0.155 \mathrm{~g}, 1.20 \mathrm{mmol}), \mathrm{Pd}(\mathrm{OAc})_{2}$ and $\mathrm{CyPF}-t$-Bu $\left(50.0 \mu \mathrm{L}\right.$ from stock solution $\left.\mathbf{A}, 5.0 \times 10^{-4} \mathrm{mmol}\right)$ and lithium bis(trimethylsilyl)amide $(0.402 \mathrm{~g}, 2.40$ $\mathrm{mmol})$ gave $257 \mathrm{mg}(98 \%)$ of $4-N$-octyl-amino-acetanilide as a yelow solid. ${ }^{1} \mathrm{H} \mathrm{NMR}\left(\mathrm{CD}_{3} \mathrm{OD}\right) \delta 7.18$ $(\mathrm{d}, J=8.5 \mathrm{~Hz}, 2 \mathrm{H}), 6.51(\mathrm{~d}, J=8.5 \mathrm{~Hz}, 2 \mathrm{H}), 2.95(\mathrm{t}, J=7.2 \mathrm{~Hz}, 2 \mathrm{H}), 1.99(\mathrm{~s}, 3 \mathrm{H}), 1.51$ (quint, $J=$ $7.2 \mathrm{~Hz}, 2 \mathrm{H}), 1.23-1.33(\mathrm{~m}, 10 \mathrm{H}), 0.83(\mathrm{t}, J=7.2 \mathrm{~Hz}, 3 \mathrm{H}) ;{ }^{13} \mathrm{C} \mathrm{NMR}\left(\mathrm{CD}_{3} \mathrm{OD}\right) \delta 171.08,147.56$, $129.43,123,29,114.00,45.35,33.01,30.62$, 30.43, 30.39, 28.32, 23.72, 23.48, 14.48. Anal. Calcd. For $\mathrm{C}_{16} \mathrm{H}_{26} \mathrm{~N}_{2} \mathrm{O}: \mathrm{C}, 73.24 ; \mathrm{H}, 9.99 ; \mathrm{N}, 10.68$. Found: C, 73.22; H, 10.10; N, 10.71.<smiles>CC(=O)Nc1ccc(NOC(C)=O)cc1</smiles> 
4-N-Octylamino-acetanilide (Table 9, entry 14). The general procedure $\mathrm{C}$ conducted with 4iodoacetanilide $(0.261 \mathrm{~g}, 1.00 \mathrm{mmol})$, octylamine $(0.155 \mathrm{~g}, 1.20 \mathrm{mmol}), \mathrm{Pd}(\mathrm{OAc})_{2}(1.1 \mathrm{mg}, 0.50$ mmol\%), CyPF- $t$-Bu (2.7 mg, $0.50 \mathrm{mmol} \%)$ and lithium bis(trimethylsilyl)amide (0.402 $\mathrm{g}, 2.40$ mmol) gave $176.2 \mathrm{mg}(67 \%)$ of 4 - $N$-octyl-amino-acetanilide as a yellow solid. $\left({ }^{1} \mathrm{H}\right.$ and ${ }^{13} \mathrm{C}$ NMR spectra are the same as those for the product in Table 9, entry 13.)<smiles>CC(=O)Nc1ccc(NOc2ccccc2)cc1</smiles>

3- $N$-cyclohexylamino-acetanilide (Table 9, entry 15) The general procedure $\mathrm{C}$ conducted with 3chloroacetanilide $(0.170 \mathrm{~g}, 1.00 \mathrm{mmol})$, cyclohexylamine $(0.119 \mathrm{~g}, 1.20 \mathrm{mmol}), \mathrm{Pd}(\mathrm{OAc})_{2}$ and CyPF- $t$ $\mathrm{Bu}\left(50.0 \mu \mathrm{L}\right.$ from stock solution $\left.\mathbf{A}, 5.0 \times 10^{-4} \mathrm{mmol}\right)$ and lithium bis(trimethylsilyl)amide $(0.402 \mathrm{~g}$, $2.40 \mathrm{mmol})$ gave $172.6 \mathrm{mg}(74 \%)$ of $3-N$-cyclohexylamino-acetanilide as a yellow solid. ${ }^{1} \mathrm{H}$ NMR $\left(\mathrm{CDCl}_{3}\right) \delta 7.81(\mathrm{~s}, 1 \mathrm{H}), 7.02(\mathrm{t}, J=6.4 \mathrm{~Hz}, 1 \mathrm{H}), 6.99(\mathrm{~s}, 1 \mathrm{H}), 6.62(\mathrm{~d}, J=6.4 \mathrm{~Hz}, 1 \mathrm{H}), 6.29(\mathrm{~d}, J=$ $6.4 \mathrm{~Hz}, 1 \mathrm{H}), 3.40$ (s, b, 1H), $3.18(\mathrm{tt}, J=8.0,3.0 \mathrm{~Hz}, 1 \mathrm{H}), 2.08$ (s, $3 \mathrm{H}), 1.98$ (dd, $J=10.0,2.0 \mathrm{~Hz}, 2$ $\mathrm{H}), 1.70(\mathrm{dt}, J=10.8,2.8 \mathrm{~Hz}, 2 \mathrm{H}), 1.59(\mathrm{dt}, J=10.0,3.0 \mathrm{~Hz}, 1 \mathrm{H}), 1.26-1.34(\mathrm{~m}, 2 \mathrm{H}), 1.04-1.23(\mathrm{~m}$, $3 \mathrm{H}) ;{ }^{13} \mathrm{C} \mathrm{NMR}\left(\mathrm{CDCl}_{3}\right) \delta 168.64,147.95,139.03,129.43,108.80,108.17,104.75,51.52,31.22,25.78$, 24.84, 24.47. Anal. Calcd. For $\mathrm{C}_{14} \mathrm{H}_{20} \mathrm{~N}_{2} \mathrm{O}: \mathrm{C}, 72.38 ; \mathrm{H}, 8.68 ; \mathrm{N}, 12.06$. Found: C, 72.24; H, 8.64; N, 11.85 .<smiles>CC(=O)Nc1cccc(NC(C)C)c1</smiles>

4- $N$-isoButylamino-benzylalcohol (Table 9, entry 16) The general procedure $\mathrm{C}$ conducted with 4bromobenzylalcohol $(0.187 \mathrm{~g}, 1.00 \mathrm{mmol})$, iso-butylamine $(87.8 \mathrm{mg}, 1.20 \mathrm{mmol}), \mathrm{Pd}(\mathrm{OAc})_{2}$ and CyPF- $t$-Bu $\left(50.0 \mu \mathrm{L}\right.$ from stock solution $\left.\mathbf{A}, 5.0 \times 10^{-4} \mathrm{mmol}\right)$ and lithium bis(trimethylsilyl)amide $(0.402 \mathrm{~g}, 2.40 \mathrm{mmol})$ gave $128.7 \mathrm{mg}(72 \%)$ of $4-\mathrm{N}$-isobutylamino-benzylalcohol as a yellow liquid. ${ }^{1} \mathrm{H}$ NMR $\left(\mathrm{CDCl}_{3}\right) \delta 7.15(\mathrm{~d}, J=8.0 \mathrm{~Hz}, 2 \mathrm{H}), 6.56(\mathrm{~d}, J=8.0 \mathrm{~Hz}, 2 \mathrm{H}), 4.50(\mathrm{~s}, 2 \mathrm{H}), 3.00-3.80(\mathrm{~s}$, br, 1 $\mathrm{H}), 2.91(\mathrm{~d}, J=7.2 \mathrm{~Hz}, 2 \mathrm{H}), 1.84-1.91(\mathrm{~m}, 1 \mathrm{H}), 1.10-2.10(\mathrm{~s}, \mathrm{br}, 1 \mathrm{H}), 0.96(\mathrm{~d}, J=7.2 \mathrm{~Hz}, 6 \mathrm{H}) ;{ }^{13} \mathrm{C}$ NMR $\left(\mathrm{CDCl}_{3}\right) \delta 148.25,129.30,128.82,112.57,65.35,51.77,27.93,20.40$.<smiles>CC(C)CNc1ccc(CO)cc1</smiles>

4- $\mathrm{N}$-isoButylamino-benzylalcohol (Table 9, entry 17) The general procedure $\mathrm{C}$ conducted with 4iodobenzylalcohol (0.234 g, $1.00 \mathrm{mmol})$, iso-butylamine $(87.8 \mathrm{mg}, 1.20 \mathrm{mmol}), \mathrm{Pd}(\mathrm{OAc})_{2}(1.1 \mathrm{mg}$, $0.50 \mathrm{mmol} \%), \mathrm{CyPF}-t$-Bu $(2.7 \mathrm{mg}, 0.50 \mathrm{mmol} \%)$ and lithium bis(trimethylsilyl)amide (0.402 $\mathrm{g}, 2.40$ $\mathrm{mmol})$ gave $84.0 \mathrm{mg}(47 \%)$ of $4-\mathrm{N}$-isobutylamino-benzylalcohol as a yellow liquid. $\left({ }^{1} \mathrm{H}\right.$ and ${ }^{13} \mathrm{C}$ NMR spectra are the same as those for the product in Table 9, entry 16.)<smiles>CC(C)CNc1ccc(CO)cc1</smiles> 
1-(4- $\mathrm{N}$-sec-Butylamino-phenyl)-ethanol. (Table 9, entry 18) The general procedure C conducted with 4-chlorophenyl-1-ethanol $(0.157 \mathrm{~g}, 1.00 \mathrm{mmol})$, sec-butylamine (87.8 $\mathrm{mg}, 1.20 \mathrm{mmol}), \mathrm{Pd}(\mathrm{OAc})_{2}$ $\left(1.1 \mathrm{mg}, 5.0 \times 10^{-3} \mathrm{mmol}\right)$, CyPF-t-Bu $\left(2.8 \mathrm{mg}, 5.0 \times 10^{-3} \mathrm{mmol}\right)$ and lithium bis(trimethylsilyl)amide $(0.402 \mathrm{~g}, 2.40 \mathrm{mmol})$ gave $160.7 \mathrm{mg}(83 \%)$ of $1-(N$-sec-butylamino-phenyl)-ethanol as a pale yellow liquid. ${ }^{1} \mathrm{H}$ NMR $\left(\mathrm{CDCl}_{3}\right) \delta 7.15(\mathrm{~d}, J=8.8 \mathrm{~Hz}, 2 \mathrm{H}), 6.54(\mathrm{~d}, J=8.0 \mathrm{~Hz}, 2 \mathrm{H}), 4.74(\mathrm{q}, J=6.6 \mathrm{~Hz}, 1$ H), $3.38(\mathrm{sext}, J=6.2 \mathrm{~Hz}, 1 \mathrm{H}), 3.00(\mathrm{~s}, \mathrm{~b}, 1 \mathrm{H}), 2.20(\mathrm{~s}, \mathrm{~b}, 1 \mathrm{H}), 1.54-1.62(\mathrm{~m}, 1 \mathrm{H}), 1.41-1.51$ (m, 1 $\mathrm{H}), 1.44(\mathrm{~d}, J=6.4 \mathrm{~Hz}, 3 \mathrm{H}), 1.16(\mathrm{~d}, J=6.0 \mathrm{~Hz}, 3 \mathrm{H}), 0.95(\mathrm{t}, J=7.4 \mathrm{~Hz}, 3 \mathrm{H}) ;{ }^{13} \mathrm{C} \mathrm{NMR}\left(\mathrm{CDCl}_{3}\right) \delta$ 147.03, 133.93, 126.56, 112.84, 69.93, 49.73, 29.46, 24.52, 20.08, 10.26. Anal. Calcd. For $\mathrm{C}_{12} \mathrm{H}_{19} \mathrm{NO}$ : C, 74.57; H, 9.91; N, 7.25. Found: C, 74.20; H, 9.86; N, 7.20.

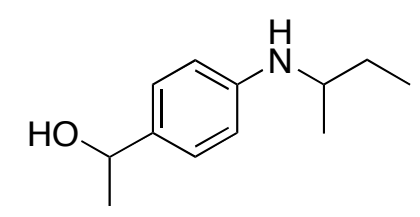

1-(4-N-octylamino-phenyl)-ethanol. (Table 9, entry 19) The general procedure $\mathrm{C}$ conducted with 4chlorophenyl-1-ethanol $(0.157 \mathrm{~g}, 1.00 \mathrm{mmol})$, octylamine $(0.155 \mathrm{~g}, 1.20 \mathrm{mmol}), \mathrm{Pd}(\mathrm{OAc})_{2}(1.1 \mathrm{mg}, 5.0$ $\left.\times 10^{-3} \mathrm{mmol}\right)$, CyPF- $t$-Bu $\left(2.8 \mathrm{mg}, 5.0 \times 10^{-3} \mathrm{mmol}\right)$ and lithium bis(trimethylsilyl)amide $(0.402 \mathrm{~g}$, $2.40 \mathrm{mmol})$ gave $219.8 \mathrm{mg}(89 \%)$ of 1 - $\left(N\right.$-octylamino-phenyl)-ethanol as a pale yellow liquid. ${ }^{1} \mathrm{H}$ $\operatorname{NMR}\left(\mathrm{CDCl}_{3}\right) \delta 7.01(\mathrm{~d}, J=8.4 \mathrm{~Hz}, 2 \mathrm{H}), 6.55(\mathrm{~d}, J=8.0 \mathrm{~Hz}, 2 \mathrm{H}), 3.77(\mathrm{t}, J=6.4 \mathrm{~Hz}, 2 \mathrm{H}), 3.40(\mathrm{~s}$, b, $1 \mathrm{H}$ ), $3.07(\mathrm{t}, J=6.8 \mathrm{~Hz}, 2 \mathrm{H}), 2.73(\mathrm{t}, J=6.4 \mathrm{~Hz}, 2 \mathrm{H}), 1.58$ (quint, $J=7.2 \mathrm{~Hz}, 2 \mathrm{H}$ ), 1.28-1.40 (m, $11 \mathrm{H}), 0.88(\mathrm{t}, J=6.8 \mathrm{~Hz}, 3 \mathrm{H}) ;{ }^{13} \mathrm{C} \mathrm{NMR}\left(\mathrm{CDCl}_{3}\right) \delta 147.19,129.76,126.56,112.97,63.92,44.19$, $38.27,31.79,29.59,29.38,29.22,27.15,22.61$, 14.04. Anal. Calcd. For $\mathrm{C}_{16} \mathrm{H}_{27} \mathrm{NO}$ : C, 77.06; H, 10.91; N, 5.62. Found: C, 77.21; H, 11.13; N, 5.61.

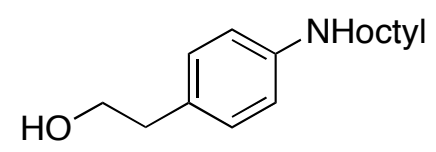

3-Hydroxy- $\boldsymbol{N}$-benzyl-aniline (Table 9, entry 20) The general procedure $\mathrm{C}$ conducted with 3chlorophenol (0.129 g, $1.00 \mathrm{mmol})$, benzylamine $(0.129 \mathrm{~g}, 1.20 \mathrm{mmol}), \mathrm{Pd}(\mathrm{OAc})_{2}\left(1.1 \mathrm{mg}, 5.0 \times 10^{-3}\right.$ $\mathrm{mmol})$, CyPF-t-Bu $\left(2.8 \mathrm{mg}, 5.0 \times 10^{-3} \mathrm{mmol}\right)$ and lithium bis(trimethylsilyl)amide $(0.402 \mathrm{~g}, 2.40$ mmol) gave $168.1 \mathrm{mg}(84 \%)$ of 3-hydroxy- $N$-benzyl-aniline as a pale yellow solid. ${ }^{1} \mathrm{H} \mathrm{NMR}\left(\mathrm{CDCl}_{3}\right) \delta$ 7.34-7.42 (m, $5 \mathrm{H}), 7.07(\mathrm{t}, J=8.0 \mathrm{~Hz}, 1 \mathrm{H}), 6.29(\mathrm{dd}, J=8.6,1.8 \mathrm{~Hz}, 1 \mathrm{H}), 6.26(\mathrm{dd}, J=8.0,2.4 \mathrm{~Hz}$, $1 \mathrm{H}), 6.16(\mathrm{t}, J=2.2 \mathrm{~Hz}, 1 \mathrm{H}), 4.50-5.50(\mathrm{~s}, \mathrm{~b}, 2 \mathrm{H}), 4.29(\mathrm{~s}, 2 \mathrm{H}) ;{ }^{13} \mathrm{C} \mathrm{NMR}\left(\mathrm{CDCl}_{3}\right) \delta 156.47,149.46$, 139.01, 130.18, 128.53, 127.44, 127.16, 106.01, 104.92, 100.12, 48.17. Anal. Calcd. For $\mathrm{C}_{13} \mathrm{H}_{13} \mathrm{NO}: \mathrm{C}$, 78.36; H, 6.58; N, 7.03. Found: C, 78.17; H, 6.51; N, 6.84 .

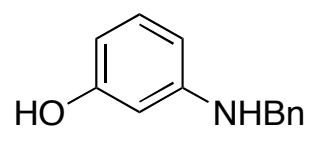

3-Hydroxy- $\boldsymbol{N}$-benzyl-aniline (Table 9, entry 21) The general procedure $\mathrm{C}$ conducted with 3bromophenol (0.172 g, $1.00 \mathrm{mmol})$, benzylamine $(0.129 \mathrm{~g}, 1.20 \mathrm{mmol}), \mathrm{Pd}(\mathrm{OAc})_{2}\left(1.1 \mathrm{mg}, 5.0 \times 10^{-3}\right.$ $\mathrm{mmol})$, CyPF- $t$-Bu $\left(2.8 \mathrm{mg}, 5.0 \times 10^{-3} \mathrm{mmol}\right)$ and lithium bis(trimethylsilyl)amide $(0.402 \mathrm{~g}, 2.40$ mmol) gave $170.1 \mathrm{mg}(85 \%)$ of 3 -hydroxy- $N$-benzyl-aniline as a pale yellow solid. $\left({ }^{1} \mathrm{H}\right.$ and ${ }^{13} \mathrm{C}$ NMR spectra are the same as those for the product in Table 9, entry 20.)

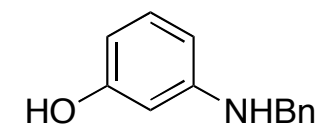


3-Hydroxy- $N$-cyclohexyl-aniline ${ }^{21}$ (Table 9, entry 22) The general procedure C conducted with 3bromophenol $(0.172 \mathrm{~g}, 1.00 \mathrm{mmol})$, cyclohexylamine $(0.119 \mathrm{~g}, 1.20 \mathrm{mmol}), \mathrm{Pd}(\mathrm{OAc})_{2}(1.1 \mathrm{mg}, 5.0 \times$ $\left.10^{-3} \mathrm{mmol}\right)$, CyPF- $t$-Bu $\left(2.8 \mathrm{mg}, 5.0 \times 10^{-3} \mathrm{mmol}\right)$ and lithium bis(trimethylsilyl)amide $(0.402 \mathrm{~g}, 2.40$ mmol) gave $154.6 \mathrm{mg}(81 \%)$ of 3-hydroxy- $N$-cyclohexyl-aniline as a pale yellow solid. ${ }^{1} \mathrm{H}$ NMR $\left(\mathrm{CDCl}_{3}\right) \delta 6.98(\mathrm{t}, J=7.6 \mathrm{~Hz}, 1 \mathrm{H}), 6.19(\mathrm{dd}, J=8.0,1.6 \mathrm{~Hz}, 1 \mathrm{H}), 6.16(\mathrm{dd}, J=8.0,1.6 \mathrm{~Hz}, 1 \mathrm{H}), 6.05$ $(\mathrm{t}, J=2.0 \mathrm{~Hz}, 1 \mathrm{H}), 4.70(\mathrm{~s}, \mathrm{br}, 2 \mathrm{H}), 3.15(\mathrm{tt}, J=10.0,4.0 \mathrm{~Hz}, 1 \mathrm{H}), 2.02(\mathrm{dd}, J=12.8,2.4 \mathrm{~Hz}, 2 \mathrm{H})$, $1.72(\mathrm{dt}, J=13.2,3.6 \mathrm{~Hz}, 2 \mathrm{H}), 1.62(\mathrm{dt}, J=12.0,3.6 \mathrm{~Hz}, 1 \mathrm{H}), 1.26-1.37$ (m, $2 \mathrm{H}), 1.04-1.23$ (m, 3 $\mathrm{H}) ;{ }^{13} \mathrm{C} \mathrm{NMR}\left(\mathrm{CDCl}_{3}\right) \delta 148.25,129.30,128.82,112.57,65.35,51.77,27.93,20.40$.

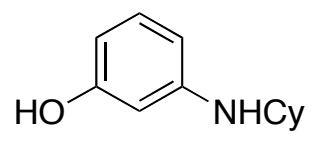

3-Hydroxy- $\boldsymbol{N}$-cyclohexyl-aniline ${ }^{21}$ (Table 9, entry 23). The general procedure $\mathrm{C}$ conducted with 3iodophenol $(0.220 \mathrm{~g}, 1.00 \mathrm{mmol})$, cyclohexylamine $(0.119 \mathrm{~g}, 1.20 \mathrm{mmol}), \mathrm{Pd}(\mathrm{OAc})_{2}(2.2 \mathrm{mg}, 1.0$ $\mathrm{mmol} \%)$, CyPF- $t$-Bu (5.5 mg, $1.0 \mathrm{mmol} \%)$ and lithium bis(trimethylsilyl)amide (0.402 $\mathrm{g}, 2.40 \mathrm{mmol})$ gave $133.8 \mathrm{mg}(70 \%)$ of 3 -hydroxy- $N$-cyclohexyl-aniline as a pale yellow solid. $\left({ }^{1} \mathrm{H}\right.$ and ${ }^{13} \mathrm{C}$ NMR spectra are the same as those for the product in Table 9, entry 22.)

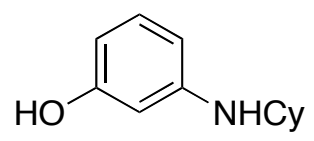

4-Hydroxy- $\boldsymbol{N}$-octyl-aniline (Table 9, entry 24) The general procedure $\mathrm{C}$ conducted with 4chlorophenol $(0.129 \mathrm{~g}, 1.00 \mathrm{mmol})$, octylamine $(0.155 \mathrm{~g}, 1.20 \mathrm{mmol}), \mathrm{Pd}(\mathrm{OAc})_{2}\left(1.1 \mathrm{mg}, 5.0 \times 10^{-3}\right.$ $\mathrm{mmol})$, CyPF- $t$-Bu $\left(2.8 \mathrm{mg}, 5.0 \times 10^{-3} \mathrm{mmol}\right)$ and lithium bis(trimethylsilyl)amide $(0.402 \mathrm{~g}, 2.40$ mmol) gave $159.6 \mathrm{mg}(72 \%)$ of 4-hydroxy- $N$-octyl-aniline as a white solid. ${ }^{1} \mathrm{H}$ NMR $\left(\mathrm{CDCl}_{3}\right) \delta 6.62$ $(\mathrm{d}, J=6.4 \mathrm{~Hz}, 2 \mathrm{H}), 6.53(\mathrm{~d}, J=6.4 \mathrm{~Hz}, 2 \mathrm{H}), 4.50-5.20(\mathrm{~s}, \mathrm{~b}, 2 \mathrm{H}), 3.02(\mathrm{t}, J=6.8 \mathrm{~Hz}, 2 \mathrm{H}), 1.56$ (quint, $J=7.0 \mathrm{~Hz}, 2 \mathrm{H}), 1.27-1.35(\mathrm{~m}, 10 \mathrm{H}), 0.88(\mathrm{t}, J=7.0 \mathrm{~Hz}, 3 \mathrm{H}) ;{ }^{13} \mathrm{C} \mathrm{NMR}\left(\mathrm{CDCl}_{3}\right) \delta 148.45$, $141.73,116.28,115.29,45.68,31.76,29.42$, 29.35, 29.19, 27.11, 22.60, 14.06. Anal. Calcd. For $\mathrm{C}_{14} \mathrm{H}_{23} \mathrm{NO}$ : C, 75.97; H, 10.47; N, 6.33. Found: C, 75.88; H, 10.57; N, 6.33.<smiles>Oc1ccc(NOc2ccccc2)cc1</smiles>

Methyl-4-(isobutylamino)-benzoate (Table 9, entry 25). The general procedure D conducted with methyl-4-chlorobenzoate $(0.1719 \mathrm{mg}, 1.00 \mathrm{mmol})$, isobutylamine $(0.119 \mathrm{~g}, 1.20 \mathrm{mmol}), \mathrm{Pd}(\mathrm{OAc})_{2}(2.2$ $\left.\mathrm{mg}, 1.0 \times 10^{-2} \mathrm{mmol}\right)$, CyPF- $t$-Bu $\left(5.5 \mathrm{mg}, 1.0 \times 10^{-2} \mathrm{mmol}\right)$ and potassium phosphate $(0.254 \mathrm{mg}, 1.40$ mmol) gave $194.4 \mathrm{mg}(94 \%)$ of methyl-4-(isobutylamino)-benzoate as a white solid. ${ }^{1} \mathrm{H} \mathrm{NMR}\left(\mathrm{CDCl}_{3}\right)$ $\delta 7.83(\mathrm{~d}, J=8.5 \mathrm{~Hz}, 2 \mathrm{H}), 6.51(\mathrm{~d}, J=8.5 \mathrm{~Hz}, 2 \mathrm{H}), 4.32(\mathrm{~s}, 1 \mathrm{H}), 3.81(\mathrm{~s}, 3 \mathrm{H}), 2.93(\mathrm{t}, J=6.5 \mathrm{~Hz}, 2$ $\mathrm{H}), 1.86$ (nonet, $J=6.8 \mathrm{~Hz}, 1 \mathrm{H}), 0.95(\mathrm{~d}, J=6.5 \mathrm{~Hz}, 6 \mathrm{H}) ;{ }^{13} \mathrm{C} \mathrm{NMR}\left(\mathrm{CDCl}_{3}\right) \delta 167.30,152.23$, 131.41, 117.54, 111.14, 51.32, 50.88, 27.84, 20.21. Anal. Calcd. For: $\mathrm{C}_{12} \mathrm{H}_{17} \mathrm{NO}_{2} \mathrm{C}, 69.54 ; \mathrm{H}, 8.27 ; \mathrm{N}$, 6.76. Found: C, 69.46; H, 8.39; N, 6.80 .<smiles>COC(=O)c1ccc(NCC(C)C)cc1</smiles> 
Ethyl-4-(sec-butylamino)-benzoate (Table 9, entry 26). The general procedure D conducted with ethyl-4-iodobenzoate $(0.276 \mathrm{mg}, 1.00 \mathrm{mmol})$, sec-butylamine $(87.8 \mathrm{mg}, 1.20 \mathrm{mmol}), \mathrm{Pd}(\mathrm{OAc})_{2}$ (4.4 mg, $2.0 \mathrm{mmol} \%)$ and CyPF-t-Bu (11.0 mg, $2.0 \mathrm{mmol} \%)$ and potassium phosphate $(0.254 \mathrm{~g}, 1.40$ mmol) gave $169.7 \mathrm{mg}(77 \%)$ of Ethyl-4-(sec-butylamino)-benzoate as a white solid. ${ }^{1} \mathrm{H} \mathrm{NMR}\left(\mathrm{CDCl}_{3}\right)$ $\delta 7.82(\mathrm{~d}, J=8.5 \mathrm{~Hz}, 2 \mathrm{H}), 6.49(\mathrm{~d}, J=8.5 \mathrm{~Hz}, 2 \mathrm{H}), 4.29(\mathrm{q}, J=7.0 \mathrm{~Hz}, 2 \mathrm{H}), 3.98(\mathrm{~d}, J=6.0 \mathrm{~Hz}, 1$ H), 3.44 (hept, $J=6.5 \mathrm{~Hz}, 1 \mathrm{H}), 1.44-1.62(\mathrm{~m}, 2 \mathrm{H}), 1.33(\mathrm{t}, J=7.0 \mathrm{~Hz}, 3 \mathrm{H}), 1.16(\mathrm{~d}, J=6.5 \mathrm{~Hz}, 3 \mathrm{H})$, $0.93(\mathrm{t}, J=7.5 \mathrm{~Hz}, 3 \mathrm{H}) ;{ }^{13} \mathrm{C} \mathrm{NMR}\left(\mathrm{CDCl}_{3}\right) \delta 166.87,151.36,131.50,117.84,111.50,60.02,49.36$, 29.43, 19.98, 14.38, 10.24. Anal. Calcd. For $\mathrm{C}_{13} \mathrm{H}_{19} \mathrm{NO}_{2}$ : C, 70.56; H, 8.65; N, 6.33. Found: C, 70.46; $\mathrm{H}, 8.64 ; \mathrm{N}, 6.23$.<smiles>CCOC(=O)c1ccc(NC(C)CC)cc1</smiles>

Methyl-3-(isobutylamino)-benzoate (Table 9, entry 27). The general procedure D conducted with methyl-3-chlorobenzoate $(0.172 \mathrm{mg}, 1.00 \mathrm{mmol})$, isobutylamine $(0.119 \mathrm{~g}, 1.20 \mathrm{mmol}), \mathrm{Pd}(\mathrm{OAc})_{2}(2.2$ $\left.\mathrm{mg}, 1.0 \times 10^{-2} \mathrm{mmol}\right)$, CyPF-t-Bu $\left(5.5 \mathrm{mg}, 1.0 \times 10^{-2} \mathrm{mmol}\right)$ and potassium phosphate $(0.254 \mathrm{mg}, 1.40$ mmol) gave $187.7 \mathrm{mg}(91 \%)$ of methyl-3-(isobutylamino)-benzoate as a white solid. ${ }^{1} \mathrm{H}$ NMR $\left(\mathrm{CDCl}_{3}\right)$ $\delta 7.26(\mathrm{~d}, J=7.6 \mathrm{~Hz}, 1 \mathrm{H}), 7.18(\mathrm{t}, J=2.0 \mathrm{~Hz}, 1 \mathrm{H}), 7.12(\mathrm{t}, J=7.6 \mathrm{~Hz}, 1 \mathrm{H}), 6.68(\mathrm{dd}, J=7.6,2.0 \mathrm{~Hz}$, $1 \mathrm{H}), 3.80(\mathrm{~s}, 3 \mathrm{H}), 3.75-3.95(\mathrm{~s}, \mathrm{br}, 1 \mathrm{H}), 2.87(\mathrm{~d}, J=6.8 \mathrm{~Hz}, 2 \mathrm{H}), 1.80$ (nonet, $J=6.8 \mathrm{~Hz}, 1 \mathrm{H}$ ), 0.90 $(\mathrm{d}, J=6.8 \mathrm{~Hz}, 6 \mathrm{H}) ;{ }^{13} \mathrm{C} \mathrm{NMR}\left(\mathrm{CDCl}_{3}\right) \delta 167.54,148.47,130.86,129.04,117.95,117.02,113.03$, 51.94, 51.55, 27.89, 20.37. Anal. Calcd. For: $\mathrm{C}_{12} \mathrm{H}_{17} \mathrm{NO}_{2} \mathrm{C}, 69.54 ; \mathrm{H}, 8.27$; N, 6.76. Found: C, 69.38; $\mathrm{H}, 8.27 ; \mathrm{N}, 6.69$.

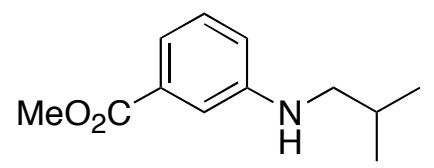

Ethyl-3-(isobutylamino)-benzoate (Table 9, entry 28). The general procedure D conducted with ethyl-3-bromobenzoate $(0.215 \mathrm{mg}, 1.00 \mathrm{mmol})$, isobutylamine $(0.119 \mathrm{~g}, 1.20 \mathrm{mmol}), \mathrm{Pd}(\mathrm{OAc})_{2}(2.2$ $\left.\mathrm{mg}, 1.0 \times 10^{-2} \mathrm{mmol}\right)$, CyPF-t-Bu $\left(5.5 \mathrm{mg}, 1.0 \times 10^{-2} \mathrm{mmol}\right)$ and potassium phosphate $(0.254 \mathrm{mg}, 1.40$ mmol) gave $167.5 \mathrm{mg}(81 \%)$ of Ethyl-3-(isobutylamino)-benzoate as a white solid. $\left({ }^{1} \mathrm{H}\right.$ and ${ }^{13} \mathrm{C}$ NMR spectra are the same as those for the product in Table 9, entry 27.)<smiles>CC(=O)c1cccc(NCC(C)C)c1</smiles>

Methyl-2-(benzylamino)-benzoate (Table 9, entry 29). The general procedure D conducted with methyl-2-chlorobenzoate $(0.172 \mathrm{mg}, 1.00 \mathrm{mmol})$, benzylamine $(0.119 \mathrm{~g}, 1.20 \mathrm{mmol}), \mathrm{Pd}(\mathrm{OAc})_{2}(4.4$ $\left.\mathrm{mg}, 2.0 \times 10^{-2} \mathrm{mmol}\right)$, CyPF- $t$-Bu $\left(11.0 \mathrm{mg}, 2.00 \times 10^{-2} \mathrm{mmol}\right)$ and potassium phosphate $(0.254 \mathrm{mg}$, $1.40 \mathrm{mmol})$ gave $197.7 \mathrm{mg}(82 \%)$ of methyl-2-(benzylamino)-benzoate as a white solid. ${ }^{1} \mathrm{H}$ NMR $\left(\mathrm{CDCl}_{3}\right) \delta 8.22(\mathrm{~s}, 1 \mathrm{H}), 7.96(\mathrm{dd}, J=8.0,2.0 \mathrm{~Hz}, 1 \mathrm{H}), 7.26-7.39(\mathrm{~m}, 6 \mathrm{H}), 6.66(\mathrm{~d}, J=8.5 \mathrm{~Hz}, 1 \mathrm{H})$, $6.62(\mathrm{td}, J=8.0,1.0 \mathrm{~Hz}, 1 \mathrm{H}), 4.47(\mathrm{~d}, J=5.5 \mathrm{~Hz}, 2 \mathrm{H}), 3.88(\mathrm{~s}, 3 \mathrm{H}) ;{ }^{13} \mathrm{C}$ NMR $\left(\mathrm{CDCl}_{3}\right) \delta 169.06$, $150.89,138.79,134.56,131.56,128.62,127.08,127.00,114.79,111.59,110.07,51.42,46.86$. 


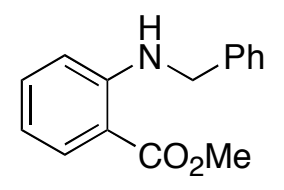

4-Nitro- $\mathrm{N}$-octyl-aniline (Table 9, entry 30). The general procedure conducted with 4-nitro-1iodobenzene $(0.249 \mathrm{mg}, 1.00 \mathrm{mmol})$, octylamine $(0.155 \mathrm{~g}, 1.20 \mathrm{mmol}), \mathrm{Pd}(\mathrm{OAc})_{2}(2.2 \mathrm{mg}, 1.0$ $\mathrm{mmol} \%)$, CyPF- $t$-Bu $(5.5 \mathrm{mg}, 1.0 \mathrm{mmol} \%)$ and potassium phosphate $(0.254 \mathrm{~g}, 1.40 \mathrm{mmol})$ gave 198.0 $\mathrm{mg}(79 \%)$ of 4-nitro- $N$-octyl-aniline as a white solid. ${ }^{1} \mathrm{H} \mathrm{NMR}\left(\mathrm{CDCl}_{3}\right) \delta 8.05(\mathrm{~d}, J=9.0 \mathrm{~Hz}, 2 \mathrm{H})$, $6.49(\mathrm{~d}, J=9.0 \mathrm{~Hz}, 2 \mathrm{H}), 4.55(\mathrm{~s}, 1 \mathrm{H}), 3.17$ (q, $J=7.0 \mathrm{~Hz}, 2 \mathrm{H}$ ), 1.62 (quint, $J=7.0 \mathrm{~Hz}, 2 \mathrm{H}$ ), 1.21 $1.39(\mathrm{~m}, 10 \mathrm{H}), 0.86(\mathrm{t}, J=7.0 \mathrm{~Hz}, 3 \mathrm{H}) ;{ }^{13} \mathrm{C} \mathrm{NMR}\left(\mathrm{CDCl}_{3}\right) \delta 153.49,137.60,126.46,110.83,43.35$, 31.70, 29.21, 29.13, 29.02, 26.92, 22.57, 14.03. Anal. Calcd. For $\mathrm{C}_{14} \mathrm{H}_{22} \mathrm{~N}_{2} \mathrm{O}_{2}: \mathrm{C}, 67.17 ; \mathrm{H}, 8.86 ; \mathrm{N}$, 11.19. Found: C, 67.09; H, 8.99; N, 11.11 .<smiles>O=[N+]([O-])c1ccc(NOc2ccccc2)cc1</smiles>

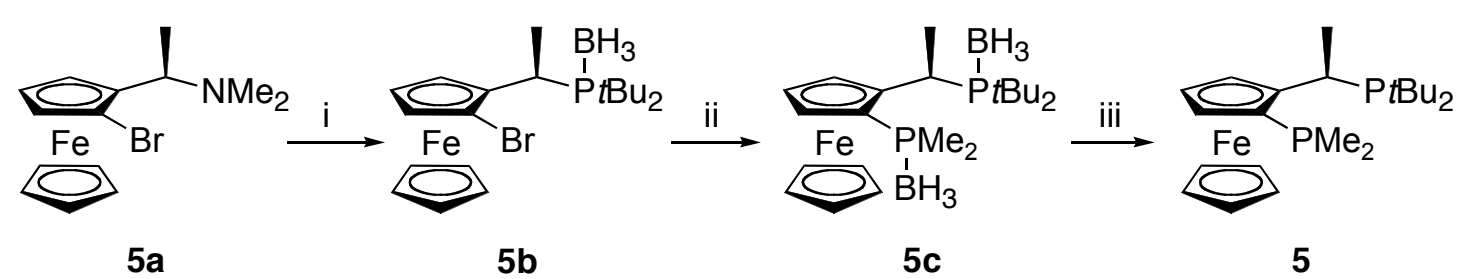

Reagents and conditions: Compound $5 \mathbf{a}$ was prepared according to the reported procedure. i. a) $\mathrm{HP} t \mathrm{Bu}_{2}, \mathrm{AcOH}, 100{ }^{\circ} \mathrm{C}, 3 \mathrm{~h}$; b) $\mathrm{BH}_{3}, \mathrm{THF}, 10 \mathrm{~min}$; ii. a) $t \mathrm{BuLi}, \mathrm{THF},{ }_{-} 78^{\circ} \mathrm{C}, 35 \mathrm{~min}, \mathrm{ClPMe}_{2}$, overnight, RT; b) $\mathrm{BH}_{3}$, THF, $10 \mathrm{~min}$; iii. Morpholine, $100^{\circ} \mathrm{C}, 4 \mathrm{~h}$.

Preparation of Racemic MePF-t-Bu 5 [CpFe $\left(\mathrm{C}_{5} \mathrm{H}_{3}\left(\mathrm{CH}(\mathrm{Me}) \mathrm{PtBu}_{2}\left(\mathrm{PMe}_{2}\right)-1,2\right]\right.$. A red solution of compound $5 \mathbf{a}(0.182 \mathrm{~g}, 0.540 \mathrm{mmol})$ and $\mathrm{HPt} \mathrm{Bu}_{2}(79.3 \mathrm{mg}, 0.540 \mathrm{mmol})$ in $2.0 \mathrm{~mL}$ acetic acid was heated at $100{ }^{\circ} \mathrm{C}$ for $3 \mathrm{~h}$. The solvent was removed under reduced pressure, and $2 \mathrm{~mL}$ of THF was added. To the solution was added $5.0 \mathrm{~mL}$ of $\mathrm{BH}_{3} \bullet \mathrm{THF}(1.0 \mathrm{M})$. The resulting solution was stirred for 10 min, after which time $5 \mathrm{~mL}$ of $\mathrm{MeOH}$ was added slowly with cooling in an ice bath to quench the excess $\mathrm{BH}_{3}$. The solvent was removed under reduced pressure, and the residue was purified by flash chromatography (hexane/ethyl acetate, 90/10) to give $231.4 \mathrm{mg}(95 \%)$ to give the product $\mathbf{5 b}$ as an orange solid. ${ }^{1} \mathrm{H}$ NMR $\left(\mathrm{C}_{6} \mathrm{D}_{6}\right) \delta$ 4.30-4.31 (m, $\left.1 \mathrm{H}\right), 4.26-4.28(\mathrm{~m}, 1 \mathrm{H}), 4.08(\mathrm{~s}, 5 \mathrm{H}), 3.87-3.88(\mathrm{~m}, 1$ H), $3.28(\mathrm{dq}, J=11.0,7.5 \mathrm{~Hz}, 1 \mathrm{H}), 1.94(\mathrm{dd}, J=12.2,7.4 \mathrm{~Hz}, 3 \mathrm{H}), 1.35(\mathrm{~d}, J=12.0 \mathrm{~Hz}, 9 \mathrm{H}), 1.26$ $(\mathrm{d}, J=12.0 \mathrm{~Hz}, 9 \mathrm{H}),-0.2-1.0(\mathrm{br}, 3 \mathrm{H}) ;{ }^{31} \mathrm{P}\left\{{ }^{1} \mathrm{H}\right\}$ NMR $\left(\mathrm{C}_{6} \mathrm{D}_{6}\right) \delta 60.3(\mathrm{~m})$. Anal. Calcd. For $\mathrm{C}_{20} \mathrm{H}_{33} \mathrm{BBrFeP}: \mathrm{C}, 53.26$; H, 7.38. Found: C, 53.47; H, 7.51.

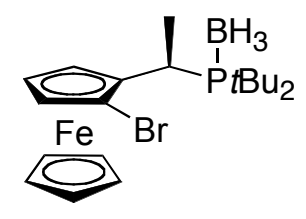

To a solution of $\mathbf{5 b}(0.143 \mathrm{mg}, 0.320 \mathrm{mmol})$ in THF $(6.0 \mathrm{~mL})$ was added a solution of $t \mathrm{BuLi}(1.5 \mathrm{M}$ in hexane) in hexane $(227 \mu \mathrm{L}, 0.340 \mathrm{mmol})$ at $\_78^{\circ} \mathrm{C}$. The resulting solution was stirred for $2 \mathrm{~h}$ at $\_78^{\circ} \mathrm{C}$. A solution of $\mathrm{ClPMe}_{2}(33.0 \mathrm{mg}, 27.0 \mu \mathrm{L}, 0.350 \mathrm{mmol})$ in $3.0 \mathrm{~mL}$ of $\mathrm{Et}_{2} \mathrm{O}$ was added by syringe. The mixture was stirred overnight, and $5.0 \mathrm{~mL}$ of $\mathrm{BH}_{3} \bullet \mathrm{THF}(1.0 \mathrm{M})$ was added. After 10 min, aqueous 
saturated $\mathrm{NaHCO}_{3}(10 \mathrm{~mL})$ was slowly added with cooling in an ice bath. The resulting organic layer and $\mathrm{Et}_{2} \mathrm{O}$ extracts from the aqueous layer were combined, washed with $2 \times 20 \mathrm{~mL}$ of water, dried over $\mathrm{MgSO}_{4}$, and concentrated to afford an organic powder. The $\mathrm{BH}_{3}$ protected compound $\mathbf{5 c}$ was purified by flash chromatography (hexane/ethyl acetate, 90/10) to give $121.4 \mathrm{mg}(85 \%)$ of the product 5c as an orange solid. ${ }^{1} \mathrm{H}$ NMR $\left(\mathrm{C}_{6} \mathrm{D}_{6}\right) \delta 4.72(\mathrm{~s}, 1 \mathrm{H}), 4.45(\mathrm{t}, J=3.0 \mathrm{~Hz}, 1 \mathrm{H}), 4.27(\mathrm{~s}, 5 \mathrm{H}), 4.16-4.17$ $(\mathrm{m}, 1 \mathrm{H}), 3.71(\mathrm{dq}, J=11.0,7.5 \mathrm{~Hz}, 1 \mathrm{H}), 2.00(\mathrm{dd}, J=11.0,7.5 \mathrm{~Hz}, 3 \mathrm{H}), 1.66(\mathrm{~d}, J=10.0 \mathrm{~Hz}, 3 \mathrm{H})$, $1.50(\mathrm{~d}, J=10.0 \mathrm{~Hz}, 3 \mathrm{H}), 1.40(, \mathrm{~d}, J=12.0 \mathrm{~Hz}, 9 \mathrm{H}), 1.27$ (d, $J=12.0 \mathrm{~Hz}, 9 \mathrm{H}),-0.5-1.3(\mathrm{br}, 6 \mathrm{H})$; ${ }^{31} \mathrm{P}\left\{{ }^{1} \mathrm{H}\right\}$ NMR $\left(\mathrm{C}_{6} \mathrm{D}_{6}\right) \delta-3.03(\mathrm{~m}), 57.3(\mathrm{~m})$.

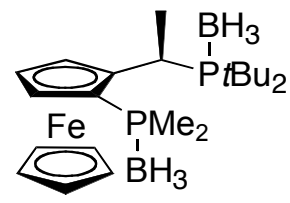

A red solution of $\mathbf{5 c}(43.0 \mathrm{mg}, 0.096 \mathrm{mmol})$ in $1.0 \mathrm{~mL}$ of morpholine was heated at $100{ }^{\circ} \mathrm{C}$ for $3 \mathrm{~h}$. The solvent was removed, and $5.0 \mathrm{~mL}$ of hexane was added. The red solution was filtered through a short plug of Celite, and the solvent was evaporated under reduced pressure. Ligand $\mathbf{5}$ was recrystalized from $5 \mathrm{~mL}$ of degassed EtOH to yield $38.1 \mathrm{mg}(95 \%)$ of orange, microcrystalline product. ${ }^{1} \mathrm{H}$ NMR

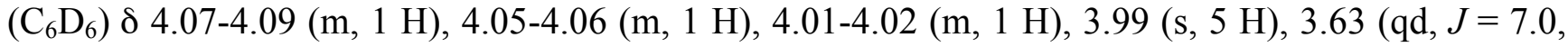
$2.5 \mathrm{~Hz}, 1 \mathrm{H}), 1.74(\mathrm{dd}, J=7.5,3.0 \mathrm{~Hz}, 3 \mathrm{H}), 1.49$ (d, $J=2.5 \mathrm{~Hz}, 3 \mathrm{H}), 1.37$ (d, $J=10.5 \mathrm{~Hz}, 9 \mathrm{H}), 1.26$ $(\mathrm{d}, J=4.5 \mathrm{~Hz}, 3 \mathrm{H}), 1.10(\mathrm{~d}, J=11.0 \mathrm{~Hz}, 9 \mathrm{H}) ;{ }^{31} \mathrm{P}\left\{{ }^{1} \mathrm{H}\right\} \mathrm{NMR}\left(\mathrm{C}_{6} \mathrm{D}_{6}\right) \delta-65.04(\mathrm{~d}, J=36 \mathrm{~Hz}), 51.96$ $(\mathrm{d}, J=36 \mathrm{~Hz}) .{ }^{13} \mathrm{C}$ NMR $\delta 101.98(\mathrm{t}, J=24.2 \mathrm{~Hz}), 79.90(\mathrm{dd}, J=16.6,3.9 \mathrm{~Hz}), 69.65(\mathrm{~d}, J=4.0 \mathrm{~Hz})$, $67.68,67.74(\mathrm{~d}, J=7.3 \mathrm{~Hz}), 67.68,33.78(\mathrm{~d}, J=29.6 \mathrm{~Hz}), 33.86(\mathrm{~d}, J=29.6 \mathrm{~Hz}), 31.86(\mathrm{~d}, J=13.6$ $\mathrm{Hz}), 31.60(\mathrm{dd}, J=13.3 \mathrm{~Hz}), 30.78(\mathrm{dd}, J=33.7,6.9 \mathrm{~Hz}), 19.52$ (t, $J=13.0 \mathrm{~Hz}), 17.02(\mathrm{~d}, J=3.0 \mathrm{~Hz})$, 3.42 (dd, $J=11.3,1.6 \mathrm{~Hz}$ ). Anal. Calcd. For $\mathrm{C}_{22} \mathrm{H}_{36} \mathrm{FeP}_{2}$ : C, 63.17; H, 8.67. Found: C, 62.94; H, 8.40.
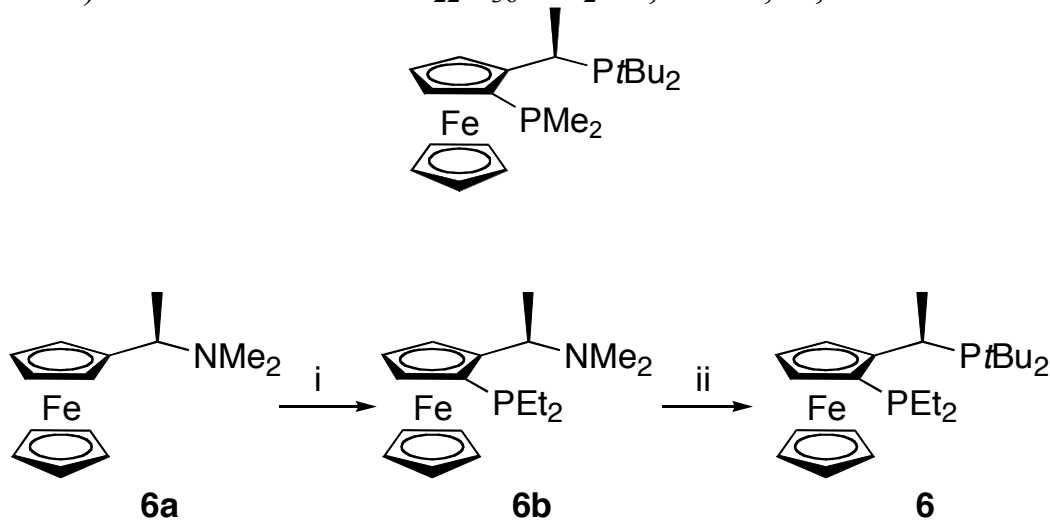

Reagents and conditions: i. a) $n \mathrm{BuLi}, \mathrm{Et}_{2} \mathrm{O}$, rt, overnight; b) $\mathrm{ClPEt}_{2}, \mathrm{Et}_{2} \mathrm{O} / \mathrm{THF}$, rt, overnight; ii. $\mathrm{HP} t \mathrm{Bu}_{2}, \mathrm{HOAc}, 100{ }^{\circ} \mathrm{C}, 1 \mathrm{~h}$.

Preparation of Racemic EtPF-t-Bu (6) [CpFe( $\mathrm{C}_{5} \mathrm{H}_{3}\left(\mathrm{CH}(\mathrm{Me}) \mathrm{PtBu} \mathbf{u}_{2}\left(\mathrm{PEt}_{2}\right)-1,2\right]$. To a solution of $6 \mathrm{a}$ $(1.43 \mathrm{~g}, 5.00 \mathrm{mmol})$ in $\mathrm{Et}_{2} \mathrm{O}(10.0 \mathrm{~mL})$ was added a solution of $n \mathrm{BuLi}(2.5 \mathrm{M}$ in hexane) in hexane $(2.10 \mathrm{~mL}, 5.25 \mathrm{mmol})$ at room temperature. The resulting solution was stirred overnight, and the clear orange solution became orange cloudy. A solution of $\mathrm{ClPEt}_{2}(0.620 \mathrm{~g}, 5.00 \mathrm{mmol})$ in $10.0 \mathrm{~mL} \mathrm{of} \mathrm{Et}_{2} \mathrm{O}$ was added through syringe. The mixture was stirred overnight. Aqueous saturated $\mathrm{NaHCO}_{3}(10 \mathrm{~mL})$ was slowly added with cooling in an ice bath. The resulting organic layer and $\mathrm{Et}_{2} \mathrm{O}$ extracts from the aqueous layer were combined, washed with $2 \times 20 \mathrm{~mL}$ of water, dried over $\mathrm{MgSO}_{4}$, and concentrated to afford an orange powder. Compound $\mathbf{6 b}$ was purified by flash chromatography (hexane/ethyl acetate, $90 / 10)$ to give $0.863 \mathrm{~g}(50 \%)$ of the product $6 \mathbf{b}$ as an orange solid. ${ }^{1} \mathrm{H}$ NMR $\left(\mathrm{C}_{6} \mathrm{D}_{6}\right) \delta 4.30(\mathrm{dd}$, $J=6.7,3.0 \mathrm{~Hz}, 1 \mathrm{H}), 4.20(\mathrm{~s}, 1 \mathrm{H}), 4.15(\mathrm{~d}, J=2.4 \mathrm{~Hz}, 1 \mathrm{H}), 4.13(\mathrm{~s}, 1 \mathrm{H}), 4.07(\mathrm{~s}, 5 \mathrm{H}), 2.20(\mathrm{~s}, 6 \mathrm{H})$, 
1.83-1.88 (m, $4 \mathrm{H}), 1.34$ (dt, $J=16.2,7.7 \mathrm{~Hz}, 3 \mathrm{H}), 1.23(\mathrm{~d}, J=6.8 \mathrm{~Hz}, 3 \mathrm{H}), 1.34$ (dt, $J=15.3,7.7$ $\mathrm{Hz}, 3 \mathrm{H}) ;{ }^{31} \mathrm{P}\left\{{ }^{1} \mathrm{H}\right\}$ NMR $\left(\mathrm{C}_{6} \mathrm{D}_{6}\right) \delta-31.6(\mathrm{~s})$.

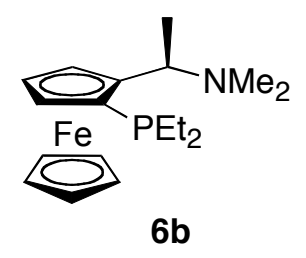

A red solution of compound $\mathbf{6 b}(0.358 \mathrm{~g}, 1.04 \mathrm{mmol})$ and $\mathrm{HP} t \mathrm{Bu}_{2}(152.5 \mathrm{mg}, 1.04 \mathrm{mmol})$ in $5.0 \mathrm{~mL}$ acetic acid was heated at $100{ }^{\circ} \mathrm{C}$ for $1 \mathrm{~h}$. The solvent was removed under reduced pressure and $1.0 \mathrm{~mL}$ of $\mathrm{Et}_{3} \mathrm{~N}$ and $10 \mathrm{~mL}$ of pentane was added. The red solution was filtered through a short plug of Celite and the solvent was evaporated. Ligand 6 was recrystalized from $5 \mathrm{~mL}$ of degassed EtOH yield 139.2 $\mathrm{mg}(30 \%)$ of orange, microcrystalline product. ${ }^{1} \mathrm{H}$ NMR $\left(\mathrm{C}_{6} \mathrm{D}_{6}\right) \delta 4.07-4.10(\mathrm{~m}, 1 \mathrm{H}), 4.02-4.04(\mathrm{~m}, 2$ H), 4.00 (s, $5 \mathrm{H}), 3.64(\mathrm{qd}, J=7.0,2.5 \mathrm{~Hz}, 1 \mathrm{H}), 2.27(\mathrm{dqd}, \mathrm{J}=14.0,7.5,1.5 \mathrm{~Hz}, 1 \mathrm{H}), 1.77-1.89$ (m, 3 $\mathrm{H}), 1.75(\mathrm{dd}, J=7.5,3.0 \mathrm{~Hz}, 3 \mathrm{H}), 1.21(\mathrm{dt}, J=16.5,8.0 \mathrm{~Hz}, 3 \mathrm{H}), 1.10(\mathrm{~d}, J=10.5 \mathrm{~Hz}, 9 \mathrm{H}), 1.05(\mathrm{dt}$, $J=9.5,7.5 \mathrm{~Hz}, 3 \mathrm{H}), 1.10(\mathrm{~d}, J=10.5 \mathrm{~Hz}, 9 \mathrm{H}) ;{ }^{31} \mathrm{P}\left\{{ }^{1} \mathrm{H}\right\} \operatorname{NMR}\left(\mathrm{C}_{6} \mathrm{D}_{6}\right) \delta-35.75(\mathrm{~d}, J=25.7 \mathrm{~Hz})$, $51.73(\mathrm{~d}, J=25.3 \mathrm{~Hz}) .{ }^{13} \mathrm{C} \mathrm{NMR}\left(\mathrm{C}_{6} \mathrm{D}_{6}\right) \delta 102.00(\mathrm{t}, J=23.0 \mathrm{~Hz}), 77.10(\mathrm{dd}, J=16.3,3.8 \mathrm{~Hz}), 70.04$ $(\mathrm{d}, J=4.7 \mathrm{~Hz}), 69.73,69.55(\mathrm{~d}, J=3.6 \mathrm{~Hz}), 67.52,34.13(\mathrm{~d}, J=30.1 \mathrm{~Hz}), 33.80(\mathrm{~d}, J=30.1 \mathrm{~Hz})$, $31.93(\mathrm{~d}, J=14.1 \mathrm{~Hz}), 31.65(\mathrm{dd}, J=7.5,2.5 \mathrm{~Hz}), 30.79(\mathrm{dd}, J=33.8,7.5 \mathrm{~Hz}), 21.10(\mathrm{~d}, J=14.5 \mathrm{~Hz})$, $20.99(\mathrm{~d}, J=14.5 \mathrm{~Hz}), 17.17(\mathrm{dd}, J=8.5,2.1 \mathrm{~Hz}), 11.82(\mathrm{~d}, J=23.9 \mathrm{~Hz}), 8.97(\mathrm{~d}, J=4.2 \mathrm{~Hz})$. Anal. Calcd. For $\mathrm{C}_{24} \mathrm{H}_{40} \mathrm{FeP}_{2}$ : C, 64.58; H, 9.03. Found: C, 64.60; H, 8.75.
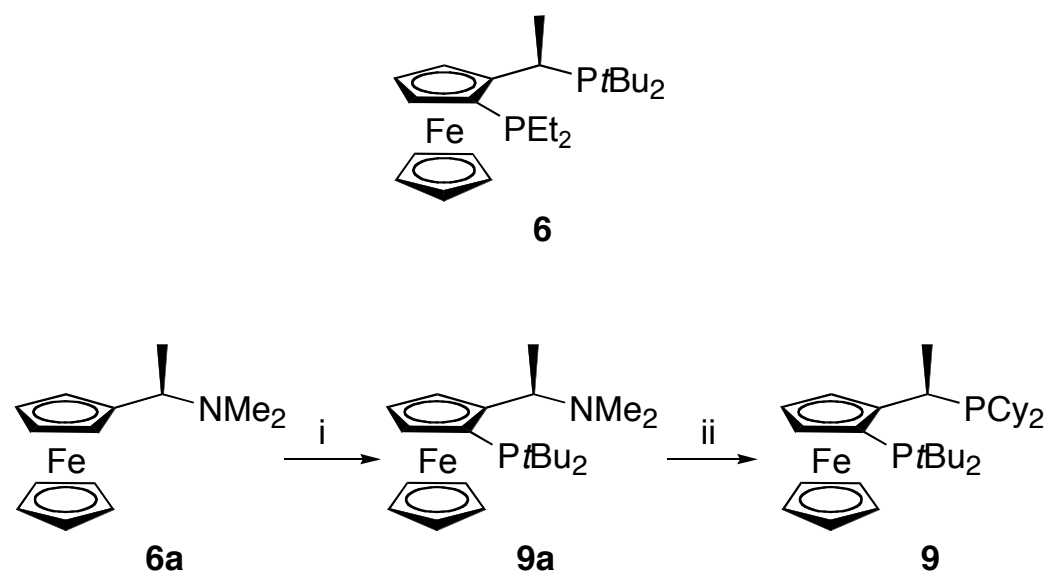

Reagents and conditions: i. a) $n \mathrm{BuLi}, \mathrm{Et}_{2} \mathrm{O}$, rt, overnight; b) $\mathrm{ClP} t \mathrm{Bu}_{2}, \mathrm{Et}_{2} \mathrm{O} / \mathrm{THF}$, rt, overnight; ii. $\mathrm{HPCy}_{2}, \mathrm{HOAc}, 100{ }^{\circ} \mathrm{C}, 1 \mathrm{~h}$.

Preparation of Racemic $t$ BuPFCy (9) $\left[\mathrm{CpFe}\left(\mathrm{C}_{5} \mathrm{H}_{3}\left(\mathrm{CH}(\mathrm{Me}) \mathrm{PCy}_{2}\left(\mathrm{PtBu}_{2}\right)-1,2\right]\right.\right.$. To a solution of $6 \mathrm{a}$ $(0.257 \mathrm{~g}, 1.00 \mathrm{mmol})$ in $\mathrm{Et}_{2} \mathrm{O}(5.0 \mathrm{~mL})$ was added a solution of $n \mathrm{BuLi}(2.5 \mathrm{M}$ in hexane) in hexane $(0.44 \mathrm{~mL}, 1.10 \mathrm{mmol})$ at room temperature. The resulting solution was stirred overnight, and the clear orange solution became orange cloudy. A solution of $\mathrm{ClP} t \mathrm{Bu}_{2}(0.180 \mathrm{~g}, 1.00 \mathrm{mmol})$ in $5.0 \mathrm{~mL} \mathrm{of} \mathrm{Et}_{2} \mathrm{O}$ was added through syringe. The mixture was stirred overnight. Aqueous saturated $\mathrm{NaHCO}_{3}(10 \mathrm{~mL})$ was slowly added with cooling in an ice bath. The resulting organic layer and $\mathrm{Et}_{2} \mathrm{O}$ extracts from the aqueous layer were combined, washed with $2 \times 20 \mathrm{~mL}$ of water, dried over $\mathrm{MgSO}_{4}$, and concentrated to afford an orange powder. Compound 9a was purified by flash chromatography (hexane/ethyl acetate, $40 / 60)$ to give $0.381 \mathrm{~g}(95 \%)$ of the product $9 \mathbf{a}$ as an orange solid. ${ }^{1} \mathrm{H} \mathrm{NMR}\left(\mathrm{CDCl}_{3}\right) \delta 4.25$ $4.30(\mathrm{~m}, 1 \mathrm{H}), 4.18-4.20(\mathrm{~m}, 1 \mathrm{H}), 4.12-4.15(\mathrm{~m}, 1 \mathrm{H}), 4.06(\mathrm{~s}, 5 \mathrm{H}), 3.95-4.02(\mathrm{~m}, 1 \mathrm{H}), 2.25(\mathrm{~s}, 6 \mathrm{H})$, $1.58(\mathrm{~d}, J=12.0 \mathrm{~Hz}, 9 \mathrm{H}), 1.28(\mathrm{~d}, J=7.2 \mathrm{~Hz}, 3 \mathrm{H}), 1.14(\mathrm{~d}, J=12.0 \mathrm{hz}, 9 \mathrm{H}) ;{ }^{31} \mathrm{P}\left\{{ }^{1} \mathrm{H}\right\} \mathrm{NMR}\left(\mathrm{CDCl}_{3}\right)$ $\delta 13.8(\mathrm{~s}) .{ }^{13} \mathrm{C}$ NMR $\left(\mathrm{C}_{6} \mathrm{D}_{6}\right) \delta 100.12(\mathrm{~d}, J=27.9 \mathrm{~Hz}), 78.88(\mathrm{~d}, J=40.6 \mathrm{~Hz}), 72.64(\mathrm{~d}, J=6.3 \mathrm{~Hz})$, 
70.44, 68.78, 68.01 (d, $J=4.6 \mathrm{~Hz}), 56.30$ (d, $J=13.1 \mathrm{~Hz}), 40.33,33.30$ (d, $J=23.3 \mathrm{~Hz}), 31.80$ (d, $J=21.2 \mathrm{~Hz}), 31.21(\mathrm{~d}, J=15.7 \mathrm{~Hz}), 30.80(\mathrm{~d}, J=14.4 \mathrm{~Hz}), 26.75(\mathrm{~d}, J=5.2 \mathrm{~Hz})$.

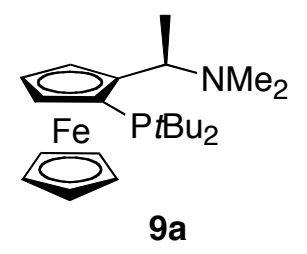

A red solution of compound 9a $(0.100 \mathrm{~g}, 0.250 \mathrm{mmol})$ and $\mathrm{HPCy}_{2}(250 \mathrm{mg}, 1.25 \mathrm{mmol})$ in $2.0 \mathrm{~mL}$ acetic acid was heated at $100{ }^{\circ} \mathrm{C}$ for $1 \mathrm{~h}$. The solvent was removed under reduced pressure, and $1.0 \mathrm{~mL}$ of $\mathrm{Et}_{3} \mathrm{~N}$ and $10 \mathrm{~mL}$ of pentane was added. The red solution was filtered through a short plug of Celite, and the solvent was evaporated. Ligand 9 was recrystalized from $5 \mathrm{~mL}$ of degassed EtOH to yield $131.2 \mathrm{mg}(95 \%)$ of orange, microcrystalline product. ${ }^{1} \mathrm{H}$ NMR $\left(\mathrm{C}_{6} \mathrm{D}_{6}\right) \delta 4.35-4.39(\mathrm{~m}, 1 \mathrm{H}), 4.13-4.15$ $(\mathrm{m}, 1 \mathrm{H}), 4.12-4.13(\mathrm{~m}, 1 \mathrm{H}), 4.09(\mathrm{~s}, 5 \mathrm{H}), 3.22(\mathrm{qd}, J=7.5,4.0 \mathrm{~Hz}, 1 \mathrm{H}), 1.85-2.10(\mathrm{~m}, 4 \mathrm{H}), 1.65-$ $1.82(\mathrm{~m}, 6 \mathrm{H}), 1.62(\mathrm{dd}, J=7.5,4.0 \mathrm{~Hz}, 3 \mathrm{H}), 1.53(\mathrm{~d}, J=12.0 \mathrm{~Hz}, 9 \mathrm{H}), 1.42-1.60(\mathrm{~m}, 5 \mathrm{H}), 1.20-1.40$ $(\mathrm{m}, 7 \mathrm{H}), 1.15(\mathrm{dt}, J=11.0 \mathrm{~Hz}, 9 \mathrm{H}) ;{ }^{31} \mathrm{P}\left\{{ }^{1} \mathrm{H}\right\}$ NMR $\left(\mathrm{C}_{6} \mathrm{D}_{6}\right) \delta 12.12(\mathrm{~d}, \mathrm{~J}=24.4 \mathrm{~Hz}), 9.52(\mathrm{~d}, \mathrm{~J}=24.4$ $\mathrm{Hz}) .{ }^{13} \mathrm{C}$ NMR $\left(\mathrm{C}_{6} \mathrm{D}_{6}\right) \delta 103.55(\mathrm{dd}, J=28.7,18.5 \mathrm{~Hz}), 78.09(\mathrm{dd}, J=37.7,1.8 \mathrm{~Hz}), 72.00(\mathrm{~d}, J=6.5$ $\mathrm{Hz}), 70.25,68.50(\mathrm{dd}, J=9.3,4.6 \mathrm{~Hz}), 68.23,34.25$ (d, $J=25.9 \mathrm{~Hz}), 33.35$ (d, $J=23.0 \mathrm{~Hz}), 32.67$ (dd, $J=19.4,1.9 \mathrm{~Hz}), 31.94(\mathrm{~d}, J=24.0 \mathrm{~Hz}), 31.77(\mathrm{~d}, J=21.2 \mathrm{~Hz}), 31.29$ (d, $J=4.6 \mathrm{~Hz}), 31.18(\mathrm{~d}, J=4.6$ $\mathrm{Hz}), 30.76(\mathrm{~d}, J=14.8 \mathrm{~Hz}), 30.21(\mathrm{~d}, J=4.6 \mathrm{~Hz}), 30.00(\mathrm{~d}, \mathrm{~J}=8.3 \mathrm{~Hz}), 28.30$ (d, $J=14.7 \mathrm{~Hz}), 28.13$ $(\mathrm{d}, J=4.6 \mathrm{~Hz}), 27.71(\mathrm{~d}, J=6.4 \mathrm{~Hz}), 27.43(\mathrm{~d}, J=12.1 \mathrm{~Hz}), 26.90,26.30(\mathrm{~d}, J=13.8 \mathrm{~Hz}), 26.11(\mathrm{~d}, J$ $=12.8 \mathrm{~Hz}), 19.54(\mathrm{~d}, \mathrm{~J}=1.8 \mathrm{~Hz})$. Anal. Calcd. For $\mathrm{C}_{32} \mathrm{H}_{52} \mathrm{FeP}_{2}$ : C, 69.31; H, 9.45. Found: C, 69.08; $\mathrm{H}$, 9.83 .
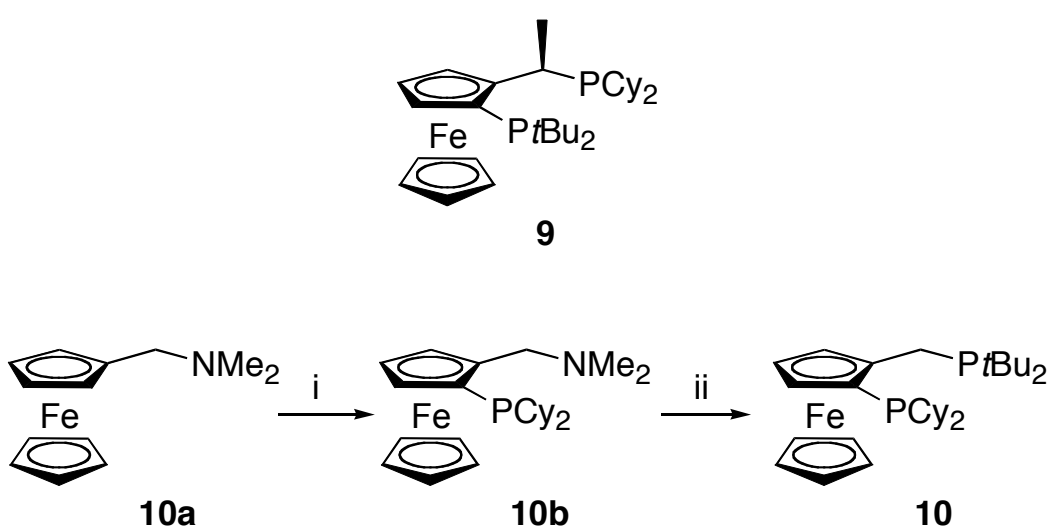

Reagents and conditions: i. a) $n \mathrm{BuLi}, \mathrm{Et}_{2} \mathrm{O}$, rt, overnight; b) $\mathrm{ClPCy}_{2}, \mathrm{Et}_{2} \mathrm{O} / \mathrm{THF}$, rt, overnight; ii. $\mathrm{HP} t \mathrm{Bu}_{2}, \mathrm{HOAc}, 100{ }^{\circ} \mathrm{C}, 1 \mathrm{~h}$.

Preparation of Racemic Ligand (10) [ $\mathrm{CpFe}\left(\mathrm{C}_{5} \mathrm{H}_{3}\left(\mathrm{CH}_{2} \mathrm{PCy}_{2}\right)\left(\mathrm{PtBu}_{2}\right)-1,2\right]$. To a solution of 10a $(0.243 \mathrm{~g}, 1.00 \mathrm{mmol})$ in $\mathrm{Et}_{2} \mathrm{O}(5.0 \mathrm{~mL})$ was added a solution of $n \mathrm{BuLi}(2.5 \mathrm{M}$ in hexane) in hexane $(0.44 \mathrm{~mL}, 1.10 \mathrm{mmol})$ at room temperature. The resulting solution was stirred overnight, and the clear orange solution became orange cloudy. A solution of $\mathrm{ClPCy}_{2}(0.233 \mathrm{~g}, 1.00 \mathrm{mmol})$ in $5.0 \mathrm{~mL}$ of Et $_{2} \mathrm{O}$ was added through syringe. The mixture was stirred overnight. Aqueous saturated $\mathrm{NaHCO}_{3}(10 \mathrm{~mL})$ was slowly added with cooling in an ice bath. The resulting organic layer and $\mathrm{Et}_{2} \mathrm{O}$ extracts from the aqueous layer were combined, washed with $2 \times 20 \mathrm{~mL}$ of water, dried over $\mathrm{MgSO}_{4}$, and concentrated to afford an orange powder. Compound $\mathbf{1 0 b}$ was purified by flash chromatography (hexane/ethyl acetate, $50 / 50)$ to give $0.350 \mathrm{~g}(83 \%)$ of the product $\mathbf{1 0 b}$ as an orange solid. ${ }^{1} \mathrm{H} \mathrm{NMR}\left(\mathrm{CDCl}_{3}\right) \delta 4.31$ $(\mathrm{s}, 1 \mathrm{H}), 4.18(\mathrm{t}, J=2.4 \mathrm{~Hz}, 1 \mathrm{H}), 4.01(\mathrm{~s}, 6 \mathrm{H}), 3.61(\mathrm{dd}, J=13.2,2.0 \mathrm{~Hz}, 1 \mathrm{H}), 3.03(\mathrm{~d}, J=12.8 \mathrm{~Hz}, 1$ 
H), 2.28-2.29 (m, $1 \mathrm{H}), 2.13(\mathrm{~s}, 6 \mathrm{H}), 1.94-1.98(\mathrm{~m}, 3 \mathrm{H}), 1.80-1.82(\mathrm{~m}, 3 \mathrm{H}), 1.54-1.71(\mathrm{~m}, 6 \mathrm{H})$, 1.15-1.39 (m, $5 \mathrm{H}), 1.04-1.06(\mathrm{~m}, 4 \mathrm{H}) .{ }^{31} \mathrm{P}\left\{{ }^{1} \mathrm{H}\right\} \mathrm{NMR}\left(\mathrm{CDCl}_{3}\right) \delta-12.13(\mathrm{~s})$.

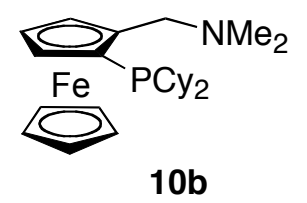

A red solution of compound $\mathbf{1 0 b}(43.9 \mathrm{mg}, 0.100 \mathrm{mmol})$ and $\mathrm{HPt} \mathrm{Bu}_{2}(16.2 \mathrm{mg}, 0.100 \mathrm{mmol})$ in $1.0 \mathrm{~mL}$ acetic acid was heated at $80{ }^{\circ} \mathrm{C}$ for $5 \mathrm{~h}$. The solvent was removed under reduced pressure, and $1.0 \mathrm{~mL}$ of $\mathrm{Et}_{3} \mathrm{~N}$ and $10 \mathrm{~mL}$ of pentane was added. The red solution was filtered through a short plug of Celite, and the solvent was evaporated. Ligand 10 was recrystalized from $5 \mathrm{~mL}$ of degassed EtOH to yield $52.2 \mathrm{mg}(95 \%)$ of orange, microcrystalline product. ${ }^{1} \mathrm{H}$ NMR $\left(\mathrm{C}_{6} \mathrm{D}_{6}\right)$ 4.65-4.68 (m, 1 H), 4.15-4.17 (m, $1 \mathrm{H}), 4.10(\mathrm{~s}, 5 \mathrm{H}), 4.05-4.08(\mathrm{~m}, 1 \mathrm{H}), 2.74(\mathrm{~d}, J=16.8 \mathrm{~Hz}, 1 \mathrm{H}), 2.63(\mathrm{dd}, J=16.4,4.4 \mathrm{~Hz}, 1 \mathrm{H})$, 2.26-2.30 (m, $1 \mathrm{H}), 2.05-2.13(\mathrm{~m}, 2 \mathrm{H}), 1.61-1.87(\mathrm{~m}, 9 \mathrm{H}), 1.32-1.48(\mathrm{~m}, 6 \mathrm{H}), 1.21(\mathrm{~d}, J=10.8 \mathrm{~Hz}, 9$ $\mathrm{H}), 1.10-1.22(\mathrm{~m}, 4 \mathrm{H}), 1.07(\mathrm{~d}, J=10.8 \mathrm{~Hz}, 9 \mathrm{H}) .{ }^{31} \mathrm{P}\left\{{ }^{1} \mathrm{H}\right\} \operatorname{NMR}\left(\mathrm{C}_{6} \mathrm{D}_{6}\right) \delta 23.39(\mathrm{~s}),-14.37(\mathrm{~s}) .{ }^{13} \mathrm{C}$ NMR $\left(\mathrm{C}_{6} \mathrm{D}_{6}\right) \delta 94.26(\mathrm{dd}, J=23.6,19.8 \mathrm{~Hz}), 79.64(\mathrm{dd}, J=20.6,3.8 \mathrm{~Hz}), 71.97(\mathrm{dd}, J=13.7,3.0 \mathrm{~Hz})$, 70.23, 70.08, 67.87, $36.56(\mathrm{dd}, J=14.6,2.3 \mathrm{~Hz}), 35.90(\mathrm{~d}, J=13.0 \mathrm{~Hz}), 33.88(\mathrm{~d}, J=23.6 \mathrm{~Hz}), 32.05$ $(\mathrm{d}, J=24.4 \mathrm{~Hz}), 31.70(\mathrm{~d}, J=22.8 \mathrm{~Hz}), 31.64(\mathrm{~d}, J=13.0 \mathrm{~Hz}), 31.39(\mathrm{~d}, J=15.3 \mathrm{~Hz}), 30.47(\mathrm{~d}, J=$ $4.6 \mathrm{~Hz}), 30.30(\mathrm{~d}, J=13.8 \mathrm{~Hz}), 30.14(\mathrm{~d}, J=14.5 \mathrm{~Hz}), 28.43(\mathrm{~d}, J=15.3 \mathrm{~Hz}), 27.99,27.91(\mathrm{~d}, J=3.0$ $\mathrm{Hz}), 27.64(\mathrm{~d}, J=10.0 \mathrm{~Hz}), 26.90,22.38(\mathrm{~d}, J=10.7 \mathrm{~Hz}), 22.13$ (d, $J=10.7 \mathrm{~Hz})$; Anal. Calcd. For $\mathrm{C}_{31} \mathrm{H}_{50} \mathrm{FeP}_{2}$ : C, 68.88; H, 9.32. Found: C, 68.85; H, 9.47.
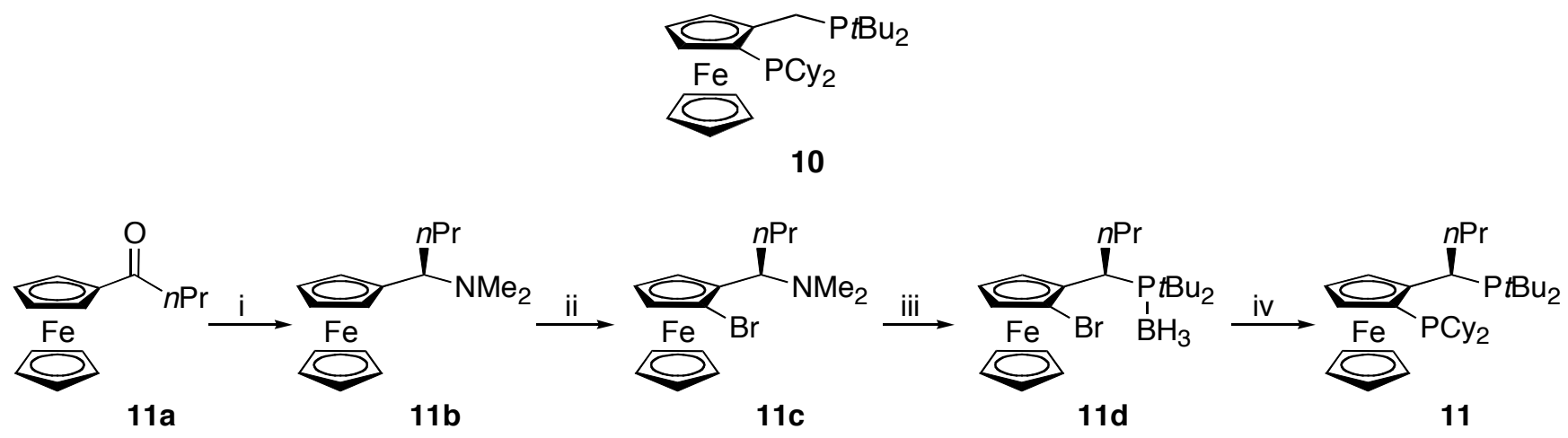

Reagents and conditions: Compound 11a was prepared according to the reported procedure. i. a) $\mathrm{HNMe}_{2}, \mathrm{Et}_{3} \mathrm{~N}, \mathrm{TiCl}_{4}, \mathrm{CH}_{2} \mathrm{Cl}_{2}$, overnight; b) $\mathrm{NaCNBH}_{3}, \mathrm{MeOH}, 0^{\circ} \mathrm{C}, 1$ h; ii. a) $\mathrm{Li}_{2} \mathrm{PdCl}_{4}, \mathrm{Et}_{3} \mathrm{~N}, \mathrm{MeOH}$, overnight; $\mathrm{Br}_{2}, \mathrm{CH}_{2} \mathrm{Cl}_{2}, 30 \mathrm{~min}$; iii. a) $\mathrm{HP} t \mathrm{Bu}_{2}, \mathrm{AcOH}, 100{ }^{\circ} \mathrm{C}, 3 \mathrm{~h}$; b) $\mathrm{BH}_{3}, \mathrm{THF}, 10 \mathrm{~min}$; ii. $t \mathrm{BuLi}$, $\mathrm{THF},{ } 78^{\circ} \mathrm{C}, 2 \mathrm{~h}, \mathrm{ClPCy}_{2}$, overnight, RT; iv. Morpholine, $100^{\circ} \mathrm{C}, 4 \mathrm{~h}$.

Preparation of Racemic Ligand (11) $\left[\mathrm{CpFe}\left(\mathrm{C}_{5} \mathrm{H}_{3}\left(\mathrm{CH}(n \mathrm{Pr}) \mathbf{P C} \mathbf{y}_{2}\right)\left(\mathbf{P t B u _ { 2 }}\right)-1,2\right]\right.$. To a dry $500 \mathrm{~mL}$ flask with septum was added compound $11 \mathrm{a}(2.56 \mathrm{~g}, 10.0 \mathrm{mmol})$, dimethylamine hydrochloride $(0.816$ $\mathrm{g}, 10.0 \mathrm{mmol})$, triethylamine $(3.0 \mathrm{~g}, 30 \mathrm{mmol})$ and $\mathrm{CH}_{2} \mathrm{Cl}_{2}(60 \mathrm{~mL})$ under nitrogen atmosphere. Titanium tetrachloride $(0.6 \mathrm{~mL}, 5.0 \mathrm{mmol})$ was added slowly via syringe. The reaction was stirred overnight and quenched with a solution of $\mathrm{NaCNBH}_{3}(1.80 \mathrm{~g}, 30.0 \mathrm{mmol})$ in $30 \mathrm{~mL}$ of $\mathrm{MeOH}$ with cooling in an ice bath. The resulting mixture was allowed to stir for $1 \mathrm{~h}$, and the $\mathrm{pH}$ was adjusted to 13 with $5 \mathrm{~N} \mathrm{NaOH}$. The resulting organic layer and EtOAc extracts from the aqueous layer were combined, washed with $2 \times 50 \mathrm{~mL}$ of water, dried over $\mathrm{MgSO}_{4}$, and concentrated to afford $\mathbf{1 1 b}$ as a red oil. This compound was used without further purification. 
A solution of $11 \mathrm{~b}(1.00 \mathrm{~g}, 3.52 \mathrm{mmol})$ in $70 \mathrm{~mL}$ of $\mathrm{MeOH}$ was added to the solution of triethyl amine $(1.06 \mathrm{~mL}, 7.00 \mathrm{mmol})$ and $\mathrm{Li}_{2} \mathrm{PdCl}_{4}(0.926 \mathrm{~g}, 3.52 \mathrm{mmol})$ in $70 \mathrm{~mL}$ of $\mathrm{MeOH}$. Orange precipitates formed after $0.5 \mathrm{~h}$, and the mixture was allowed to stir overnight. The precipitate was filtered and washed with $\mathrm{MeOH}$. The resulting orange solid was dissolved in $60.0 \mathrm{~mL}$ of $\mathrm{CH}_{2} \mathrm{Cl}_{2}$, and bromine $(0.2 \mathrm{~mL})$ in $70 \mathrm{~mL}$ of $\mathrm{CH}_{2} \mathrm{Cl}_{2}$ was added dropwise. After stirring for $30 \mathrm{~min}$, the solvent was removed under reduced pressure, and the residue was purified by flash chromatography (hexane/triethylamine, 80/20) to give $350 \mathrm{mg}(40 \%)$ of the desired compound 11c as an red oil. ${ }^{1} \mathrm{H}$ $\operatorname{NMR}\left(\mathrm{CDCl}_{3}\right) \delta 4.43(\mathrm{dd}, J=2.0,1.0 \mathrm{~Hz}, 1 \mathrm{H}), 4.13(\mathrm{~s}, 5 \mathrm{H}), 4.10(\mathrm{t}, J=2.5 \mathrm{~Hz}, 1 \mathrm{H}), 3.97(\mathrm{dd}, J=$ 2.5, $1.0 \mathrm{~Hz}, 1 \mathrm{H}), 3.60(\mathrm{dd}, J=11.0,3.5 \mathrm{~Hz}, 1 \mathrm{H}), 2.03(\mathrm{~s}, 6 \mathrm{H}), 1.93-2.01(\mathrm{~m}, 1 \mathrm{H}), 1.77-1.85(\mathrm{~m}, 1$ $\mathrm{H}), 1.61-1.68(\mathrm{~m}, 1 \mathrm{H}), 1.49-1.56(\mathrm{~m}, 1 \mathrm{H}), 1.04(\mathrm{t}, J=7.5 \mathrm{~Hz}, 3 \mathrm{H}) ;{ }^{13} \mathrm{C} \mathrm{NMR}\left(\mathrm{CDCl}_{3}\right) \delta 85.79,79.51$, 71.14, 69.47, 65.68, 65.50, 59.44, 40.89, 35.01, 20.57, 14.25. MS (m/e) 318 (100), $320(100)\left(\mathrm{M}^{+}-\right.$ $\mathrm{NMe}_{2}$ ).

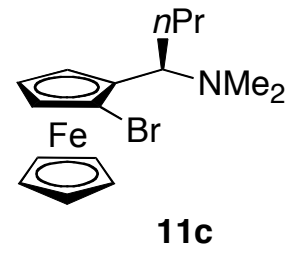

A red solution of compound $11 \mathrm{c}(0.273 \mathrm{~g}, 0.750 \mathrm{mmol})$ and $\mathrm{HPt} \mathrm{Bu}_{2}(0.122 \mathrm{~g}, 0.750 \mathrm{mmol})$ in $5.0 \mathrm{~mL}$ acetic acid was heated at $100{ }^{\circ} \mathrm{C}$ for $1 \mathrm{~h}$. The solvent was removed under reduced pressure, and $2 \mathrm{~mL}$ of THF was added. To the solution was added $5.0 \mathrm{~mL}$ of $\mathrm{BH}_{3} \bullet \mathrm{THF}(1.0 \mathrm{M})$. The resulting solution was stirred for $10 \mathrm{~min}$, and $5 \mathrm{~mL}$ of $\mathrm{MeOH}$ was added slowly with cooling in an ice bath to quench the excess $\mathrm{BH}_{3}$. The solvent was removed under reduced pressure, and the residue was purified by flash chromatography (hexane/ethyl acetate, $30 / 70)$ to give $341.3 \mathrm{mg}(95 \%)$ of the product 11d as an orange solid. ${ }^{1} \mathrm{H} \mathrm{NMR}\left(\mathrm{CDCl}_{3}\right) \delta 4.28(\mathrm{~s}, 1 \mathrm{H}), 4.15(\mathrm{~s}, 1 \mathrm{H}), 4.00(\mathrm{~s}, 6 \mathrm{H}), 2.55-2.59(\mathrm{~m}, 1 \mathrm{H}), 2.30-2.33(\mathrm{~m}$, $1 \mathrm{H}), 1.51-1.87(\mathrm{~m}, 3 \mathrm{H}), 1.66(\mathrm{~d}, J=12.3 \mathrm{~Hz}, 9 \mathrm{H}), 1.01(\mathrm{~d}, J=12.3 \mathrm{~Hz}, 9 \mathrm{H}), 0.86(\mathrm{t}, J=7.2 \mathrm{~Hz}, 3$ $\mathrm{H})$, -0.5-0.8 (m, $3 \mathrm{H}) ;{ }^{31} \mathrm{P}\left\{{ }^{1} \mathrm{H}\right\}$ NMR $\left(\mathrm{CDCl}_{3}\right) \delta 46.4(\mathrm{~m})$. Anal. Calcd. For $\mathrm{C}_{22} \mathrm{H}_{37} \mathrm{BBrFeP}$ : C, 55.16; H, 7.78. Found: C, 55.45; H, 7.89.

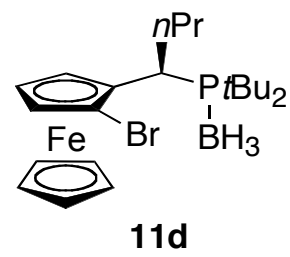

To a solution of $11 \mathrm{~d}(0.149 \mathrm{~g}, 0.310 \mathrm{mmol})$ in THF $(6.0 \mathrm{~mL})$ was added a solution of $t \mathrm{BuLi}(1.5 \mathrm{M}$ in hexane $)$ in hexane $(227 \mu \mathrm{L}, 0.340 \mathrm{mmol})$ at $\_78^{\circ} \mathrm{C}$. The resulting solution was stirred for $2 \mathrm{~h}$ at $\_78^{\circ} \mathrm{C}$. A solution of $\mathrm{ClPCy}_{2}(79.3 \mathrm{~g}, 0.340 \mathrm{mmol})$ in $3.0 \mathrm{~mL}$ of $\mathrm{Et}_{2} \mathrm{O}$ was added through syringe. The mixture was stirred overnight. Aqueous saturated $\mathrm{NaHCO}_{3}(10 \mathrm{~mL})$ was slowly added with cooling in an ice bath. The resulting organic layer and $\mathrm{Et}_{2} \mathrm{O}$ extracts from the aqueous layer were combined, washed with $2 \times 20 \mathrm{~mL}$ of water, dried over $\mathrm{MgSO}_{4}$, and concentrated to afford an organic powder. The $\mathrm{BH}_{3}$ protected compound $\mathbf{1 1} \cdot \mathrm{BH}_{3}$ was purified by flash chromatography (hexane/ethyl acetate, 95/5) to give $157.2 \mathrm{mg}(85 \%)$ of the product as an orange solid. ${ }^{1} \mathrm{H} \mathrm{NMR}\left(\mathrm{CDCl}_{3}\right) \delta 4.55-5.59(\mathrm{~m}, 1 \mathrm{H}), 4.24(\mathrm{t}$, $J=2.5 \mathrm{~Hz}, 1 \mathrm{H}), 4.18-4.20(\mathrm{~m}, 1 \mathrm{H}), 4.08(\mathrm{~s}, 5 \mathrm{H}), 3.20(\mathrm{dt}, J=12.0,9.5 \mathrm{~Hz}, 1 \mathrm{H}), 2.31-2.41(\mathrm{~m}, 2 \mathrm{H})$, 2.15-2.23 (m, $1 \mathrm{H}), 1.98-2.05(\mathrm{~m}, 3 \mathrm{H}), 1.61-1.87$ (m, $6 \mathrm{H}), 1.32-1.54(\mathrm{~m}, 6 \mathrm{H}), 1.29(\mathrm{~d}, J=12.0 \mathrm{~Hz}, 9$ $\mathrm{H}), 1.22(\mathrm{~d}, J=12.5 \mathrm{~Hz}, 9 \mathrm{H}), 1.12-1.32(\mathrm{~m}, 5 \mathrm{H}), 1.00-1.10(\mathrm{~m}, 3 \mathrm{H}), 0.98(\mathrm{t}, J=7.0 \mathrm{~Hz}, 3 \mathrm{H}),-0.50-$ $0.80(\mathrm{~m}, 3 \mathrm{H}) ;{ }^{31} \mathrm{P}\left\{{ }^{1} \mathrm{H}\right\}$ NMR $\left(\mathrm{CDCl}_{3}\right) \delta-20.38(\mathrm{~s}), 61.08(\mathrm{~m}) .{ }^{13} \mathrm{C} \mathrm{NMR}\left(\mathrm{CDCl}_{3}\right) \delta 99.83(\mathrm{dd}, J=$ 28.0, 4.2 Hz), 84.78 (dd, $J=23.9,4.7 \mathrm{~Hz}), 71.45$ (d, $J=4.7 \mathrm{~Hz}), 70.94$ (t, $J=3.1 \mathrm{~Hz}), 69.50,67.45$, 
$41.42(\mathrm{~d}, J=13.5 \mathrm{~Hz}), 40.47,34.98(\mathrm{~d}, J=23.3 \mathrm{~Hz}), 34.27-34.59$ (4 C overlap), $32.88(\mathrm{~d}, J=14.8$

$\mathrm{Hz}), 32.50$ (dd, $J=20.6,15.1 \mathrm{~Hz}), 31.34$ (d, $J=13.0 \mathrm{~Hz}), 30.14,29.19$ (d, $J=17.9 \mathrm{~Hz}), 29.03,28.25$ $(\mathrm{d}, J=2.9 \mathrm{~Hz}), 27.73(\mathrm{~d}, J=3.1 \mathrm{~Hz}), 27.60(\mathrm{~d}, J=10.9 \mathrm{~Hz}), 27.31(\mathrm{~d}, J=11.3 \mathrm{~Hz}), 26.90(\mathrm{~d}, J=2.8$ $\mathrm{Hz}), 24.56(\mathrm{~d}, J=3.3 \mathrm{~Hz}), 24.49(\mathrm{~d}, J=3.3 \mathrm{~Hz}), 14.43$.

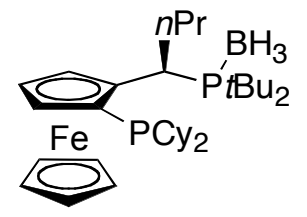

$11 \cdot \mathrm{BH} 3$

A red solution of $\mathbf{1 1} \cdot \mathrm{BH}_{3}(50 \mathrm{mg}, 0.083 \mathrm{mmol})$ in $1.0 \mathrm{~mL}$ of morpholine was heated at $100{ }^{\circ} \mathrm{C}$ for $1 \mathrm{~h}$. The solvent was removed and $5.0 \mathrm{~mL}$ of hexane was added. The red solution was filtered through a short plug of Celite, and the solvent was evaporated under reduced pressure. Recrystallization of the residue from $3.0 \mathrm{~mL}$ of degassed $\mathrm{EtOH}$ yields $46.0 \mathrm{mg}(95 \%)$ of ligand 11 as orange, microcrystalline product. ${ }^{1} \mathrm{H}$ NMR $\left(\mathrm{C}_{6} \mathrm{D}_{6}\right) \delta$ 4.24-5.27 (m, $\left.1 \mathrm{H}\right), 4.08(\mathrm{~s}, 6 \mathrm{H}), 4.07-4.09(\mathrm{~m}, 1 \mathrm{H}), 3.24-3.29(\mathrm{~m}, 1 \mathrm{H})$, 2.22-2.25 (m, $3 \mathrm{H}), 2.12-2.24(\mathrm{~m}, 2 \mathrm{H}), 2.04-2.13(\mathrm{~m}, 2 \mathrm{H}), 1.90-2.03(\mathrm{~m}, 1 \mathrm{H}), 1.45-1.87(\mathrm{~m}, 13 \mathrm{H})$, $1.39(\mathrm{~d}, J=10.0 \mathrm{~Hz}, 9 \mathrm{H}), 1.22(\mathrm{~d}, J=12.5 \mathrm{~Hz}, 9 \mathrm{H}), 1.12-1.32(\mathrm{~m}, 5 \mathrm{H}), 1.05(\mathrm{t}, J=7.0 \mathrm{~Hz}, 3 \mathrm{H}) ;{ }^{31} \mathrm{P}$ $\left\{{ }^{1} \mathrm{H}\right\} \operatorname{NMR}\left(\mathrm{C}_{6} \mathrm{D}_{6}\right) \delta-17.66(\mathrm{~d}, J=31.9 \mathrm{~Hz}), 56.79(\mathrm{~d}, J=31.5 \mathrm{~Hz}) .{ }^{13} \mathrm{C} \mathrm{NMR}\left(\mathrm{C}_{6} \mathrm{D}_{6}\right) \delta 102.93(\mathrm{dd}, J$ $=26.0,23.6 \mathrm{~Hz}), 80.00(\mathrm{dd}, J=24.7,3.0 \mathrm{~Hz}), 72.09(\mathrm{~d}, J=4.3 \mathrm{~Hz}), 69.69,69.08(\mathrm{t}, J=3.6 \mathrm{~Hz}), 67.49$, $39.30(\mathrm{~d}, J=16.0,3.4 \mathrm{~Hz}), 36.51(\mathrm{~d}, J=1.7 \mathrm{~Hz}), 35.97(\mathrm{~d}, J=6.2 \mathrm{~Hz}), 35.82(\mathrm{~d}, J=6.6 \mathrm{~Hz}), 35.52(\mathrm{~d}$, $J=7.0 \mathrm{~Hz}), 35.16(\mathrm{~d}, J=7.5 \mathrm{~Hz}), 33.92-3431$ (2 C overlap), 32.50 (d, $J=15.1 \mathrm{~Hz}), 31.62-32.20(3 \mathrm{C}$ overlap), 30.12, $28.92(\mathrm{~d}, J=16.7 \mathrm{~Hz}), 28.45$ (d, $J=10.0 \mathrm{~Hz}), 28.13(\mathrm{~d}, J=3.2 \mathrm{~Hz}), 27.95(\mathrm{~d}, J=13.0$ $\mathrm{Hz}), 27.01$ (d, $J=23.1 \mathrm{~Hz}), 24.75,15.01$.

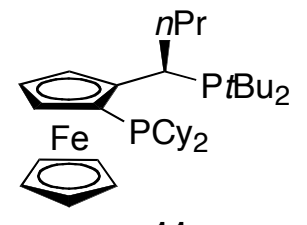

11

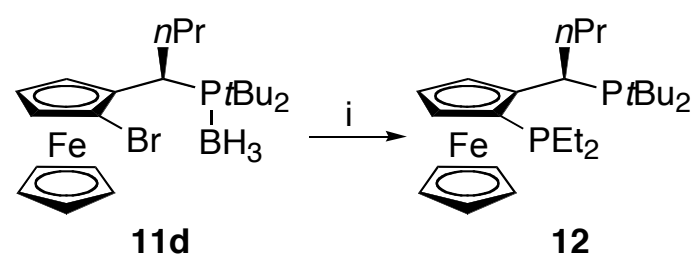

Reagents and conditions: i. a) $n \mathrm{BuLi}, \mathrm{THF},{ }_{-} 78^{\circ} \mathrm{C}, 2 \mathrm{~h}, \mathrm{ClPEt}_{2}$, overnight, RT; iv. Morpholine, $100{ }^{\circ} \mathrm{C}$, $1 \mathrm{~h}$.

To a solution of $\mathbf{1 1 d}(90.2 \mathrm{mg}, 0.200 \mathrm{mmol})$ in THF $(1.0 \mathrm{~mL})$ was added a solution of $n \mathrm{BuLi}(1.6 \mathrm{M}$ in hexane) in hexane $(133 \mu \mathrm{L}, 0.220 \mathrm{mmol})$ at $\_78^{\circ} \mathrm{C}$. The resulting solution was stirred for $2 \mathrm{~h}$ at $\_78^{\circ} \mathrm{C}$. A solution of $\mathrm{ClPEt}_{2}(24.9 \mathrm{~g}, 0.200 \mathrm{mmol})$ in $2.0 \mathrm{~mL}$ of $\mathrm{Et}_{2} \mathrm{O}$ was added through syringe. The mixture was stirred overnight. Aqueous saturated $\mathrm{NaHCO}_{3}(10 \mathrm{~mL})$ was slowly added with cooling in an ice bath. The resulting organic layer and $\mathrm{Et}_{2} \mathrm{O}$ extracts from the aqueous layer were combined, washed with $2 \times 20 \mathrm{~mL}$ of water, dried over $\mathrm{MgSO}_{4}$, and concentrated to afford an organic powder. A red solution of the organic powder in $1.0 \mathrm{~mL}$ of morpholine was heated at $100{ }^{\circ} \mathrm{C}$ for $1 \mathrm{~h}$. The solvent was removed and $5.0 \mathrm{~mL}$ of hexane was added. The red solution was filtered through a short plug of Celite, and the solvent was evaporated under reduced pressure. Recrystallization of the residue from $2.0 \mathrm{~mL}$ 
of degassed EtOH yields $76.0 \mathrm{mg}(80 \%)$ of ligand 12 as orange, microcrystalline product. ${ }^{1} \mathrm{H}$ NMR $\left(\mathrm{C}_{6} \mathrm{D}_{6}\right) \delta$ 4.00-4.08 (m, $\left.3 \mathrm{H}\right), 3.98(\mathrm{~s}, 5 \mathrm{H}), 3.35-3.45(\mathrm{~m}, 1 \mathrm{H}), 2.08-2.32(\mathrm{~m}, 3 \mathrm{H}), 1.70-2.06(\mathrm{~m}, 4 \mathrm{H})$, 1.46-1.66 (m, $1 \mathrm{H}), 1.34(\mathrm{~d}, J=10.0 \mathrm{~Hz}, 9 \mathrm{H}), 1.20(\mathrm{dt}, J=8.0,7.6 \mathrm{~Hz}, 2 \mathrm{H}), 0.95-1.18(\mathrm{~m}, 7 \mathrm{H}), 1.04$ $(\mathrm{t}, J=10.0 \mathrm{~Hz}, 9 \mathrm{H}) ;{ }^{31} \mathrm{P}\left\{{ }^{1} \mathrm{H}\right\} \operatorname{NMR}\left(\mathrm{C}_{6} \mathrm{D}_{6}\right) \delta-35.55(\mathrm{~d}, J=54.0 \mathrm{~Hz}), 60.45(\mathrm{~d}, J=54.0 \mathrm{~Hz}) .{ }^{13} \mathrm{C}$ $\operatorname{NMR}\left(\mathrm{C}_{6} \mathrm{D}_{6}\right) \delta 100.44(\mathrm{t}, J=23.6 \mathrm{~Hz}), 76.66(\mathrm{dd}, J=18.3,6.0 \mathrm{~Hz}), 70.41(\mathrm{~d}, J=4.6 \mathrm{~Hz}), 70.02$, 68.74, 67.02, 36.31 (d, $J=33.6 \mathrm{~Hz}), 34.47,34.10(\mathrm{dd}, J=36.6,3.0 \mathrm{~Hz}), 33.99(\mathrm{~d}, J=32.1 \mathrm{~Hz}), 31.97$ $(\mathrm{d}, J=15.3 \mathrm{~Hz}), 31.69$ (d, $J=13.8 \mathrm{~Hz}), 24.39,21.74(\mathrm{t}, J=13.8 \mathrm{~Hz}), 18.33$ (d, $J=9.9,4.6 \mathrm{~Hz}), 15.13$, $11.95(\mathrm{~d}, J=23.7 \mathrm{~Hz}), 9.64(\mathrm{~d}, J=6.0 \mathrm{~Hz})$. Anal. Calcd. For $\mathrm{C}_{26} \mathrm{H}_{44} \mathrm{FeP}: \mathrm{C}, 65.82 ; \mathrm{H}, 9.35$. Found: C, $65.94 ; \mathrm{H}, 9.13$.
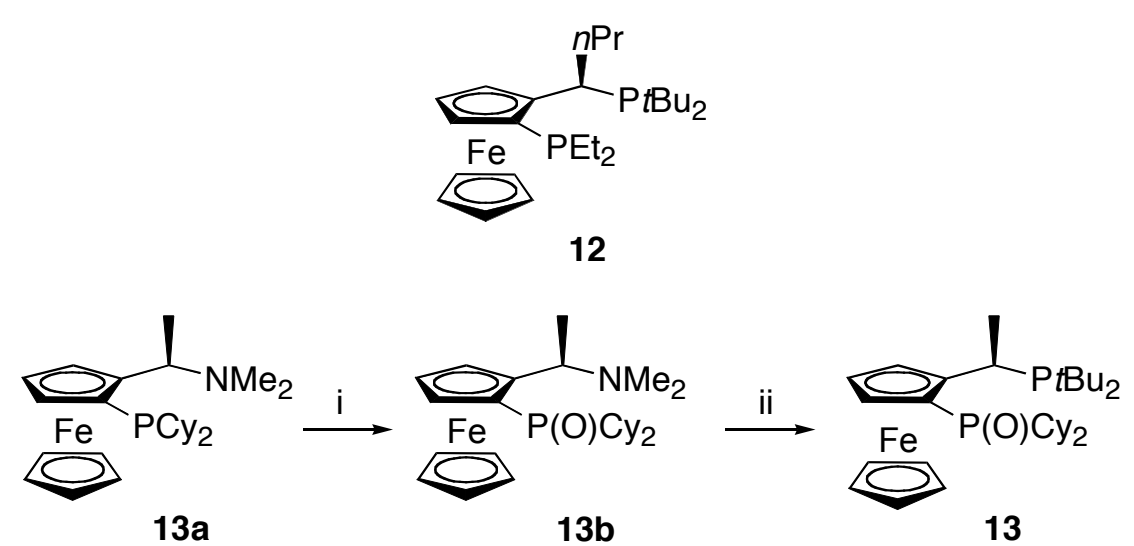

Reagents and conditions: i. a) $\mathrm{H}_{2} \mathrm{O}_{2}, \mathrm{MeOH}$, room temperature, $1 \mathrm{~h}$; ii. $\mathrm{HP} t \mathrm{Bu}_{2}, \mathrm{HOAc}, 100{ }^{\circ} \mathrm{C}, 1 \mathrm{~h}$.

Preparation of Racemic Ligand 13 [ $\mathrm{CpFe}\left(\mathrm{C}_{5} \mathrm{H}_{3}\left(\mathrm{CH}(\mathrm{Me}) \mathrm{P}(\mathrm{O}) \mathrm{Cy}_{2}\right)\left(\mathrm{PtBu}_{2}\right)-1,2\right]$. To a solution of $13 \mathrm{a}(88.0 \mathrm{mg}, 0.200 \mathrm{mmol})$ in $3 \mathrm{~mL} \mathrm{MeOH}$, one drop of $30 \% \mathrm{H}_{2} \mathrm{O}_{2}$ was added at $0{ }^{\circ} \mathrm{C}$ and stirred for 1 $\mathrm{h}$ at room temperature. The product was partitioned between $30 \mathrm{~mL} \mathrm{CHCl}$ and $10 \mathrm{~mL} \mathrm{H}_{2} \mathrm{O}$. The phase was separated, and the organic layer was washed with brine $(20 \mathrm{~mL})$, dried over $\mathrm{MgSO}_{4}$ and evaporated in vacuo to give $90.0 \mathrm{mg}(99 \%) \mathbf{1 3 b}$ as an orange powder. ${ }^{1} \mathrm{H} \mathrm{NMR}\left(\mathrm{CDCl}_{3}\right) \delta 4.99(\mathrm{~s}, 1$ $\mathrm{H}), 4.53(\mathrm{q}, J=6.7,1 \mathrm{H}), 4.47-4.50(\mathrm{~m}, 1 \mathrm{H}), 4.22(\mathrm{~s}, 5 \mathrm{H}), 4.11-4.15(\mathrm{~m}, 1 \mathrm{H}), 2.51(\mathrm{~s}, 3 \mathrm{H}), 2.32(\mathrm{~s}, 3$ $\mathrm{H}), 1.86(\mathrm{~d}, J=6.8 \mathrm{~Hz}, 3 \mathrm{H}), 1.00-2.30\left(\mathrm{~m}, 22 \mathrm{H}\right.$ from Cy); ${ }^{31} \mathrm{P}\left\{{ }^{1} \mathrm{H}\right\} \operatorname{NMR}\left(\mathrm{C}_{6} \mathrm{D}_{6}\right) \delta 49.29(\mathrm{~s})$.

A red solution of compound $\mathbf{1 3 b}(90.0 \mathrm{mg}, 0.200 \mathrm{mmol})$ and $\mathrm{HP} t \mathrm{Bu}_{2}(32.4 \mathrm{mg}, 0.200 \mathrm{mmol})$ in $2.0 \mathrm{~mL}$ acetic acid was heated at $100{ }^{\circ} \mathrm{C}$ for $30 \mathrm{~h}$. The solvent was removed under reduced pressure, and 1.0 $\mathrm{mL}$ of $\mathrm{Et}_{3} \mathrm{~N}$ and $10 \mathrm{~mL}$ of pentane was added. The red solution was filtered through a short plug of Celite, and the solvent was evaporated. Ligand 13 was recrystalized from $5 \mathrm{~mL}$ of degassed EtOH to give $82.2 \mathrm{mg}(73 \%)$ of orange, microcrystalline product. ${ }^{1} \mathrm{H} \mathrm{NMR}\left(\mathrm{C}_{6} \mathrm{D}_{6}\right) \delta 4.25(\mathrm{~s}, 5 \mathrm{H}), 4.13-4.16(\mathrm{~m}$, $1 \mathrm{H}), 4.03-4.06(\mathrm{~m}, 1 \mathrm{H}), 3.99-4.02(\mathrm{~m}, 1 \mathrm{H}), 3.67(\mathrm{dq}, J=22.4,10.4 \mathrm{~Hz}, 1 \mathrm{H}), 2.84-2.94(\mathrm{~m}, 1 \mathrm{H})$, 2.61-2.66 (m, $1 \mathrm{H}), 2.37-2.50(\mathrm{~m}, 1 \mathrm{H}), 1.75(\mathrm{dd}, J=7.6,3.2 \mathrm{~Hz}, 3 \mathrm{H}), 1.44(\mathrm{~d}, J=10.4 \mathrm{~Hz}, 9 \mathrm{H}), 1.15$ $(\mathrm{d}, J=10.8 \mathrm{~Hz}, 9 \mathrm{H}), 1.20-2.03(\mathrm{~m}, 19 \mathrm{H}$ from $\mathrm{PCy} 2) ;{ }^{31} \mathrm{P}\left\{{ }^{1} \mathrm{H}\right\} \mathrm{NMR}\left(\mathrm{C}_{6} \mathrm{D}_{6}\right) \delta 49.68(\mathrm{~s}), 42.50(\mathrm{~s}) ;$ ). ${ }^{13} \mathrm{C}$ NMR $\left(\mathrm{C}_{6} \mathrm{D}_{6}\right) \delta 101.62(\mathrm{dd}, J=22.9,12.9 \mathrm{~Hz}), 74.17(\mathrm{dd}, J=93.1,3.1 \mathrm{~Hz}), 70.89(\mathrm{~d}, J=12,2 \mathrm{~Hz})$, $70.56,70.38(\mathrm{~d}, J=8.3 \mathrm{~Hz}), 68.34(\mathrm{~d}, J=10.0 \mathrm{~Hz}), 42.83(\mathrm{~d}, J=64.8 \mathrm{~Hz}), 42.39(\mathrm{~d}, J=21.42 \mathrm{~Hz})$, $41.72(\mathrm{~d}, J=21.32 \mathrm{~Hz}), 34.18(\mathrm{~d}, J=33.6 \mathrm{~Hz}), 33.32(\mathrm{~d}, J=29.8 \mathrm{~Hz}), 32.14(\mathrm{~d}, J=14.5 \mathrm{~Hz}), 31.75$ $(\mathrm{d}, J=13.7 \mathrm{~Hz}), 30.11,29.79(\mathrm{~d}, J=35.1 \mathrm{~Hz}), 28.26(\mathrm{~d}, J=11.4 \mathrm{~Hz}), 27.94(\mathrm{~d}, J=12.3 \mathrm{~Hz}), 27.70$ (d, $J=3.1 \mathrm{~Hz}), 27.58,27.34,27.21,27.04,26.78,26.66(\mathrm{~d}, J=1.5 \mathrm{~Hz})$.

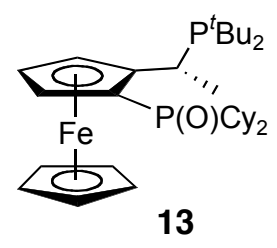




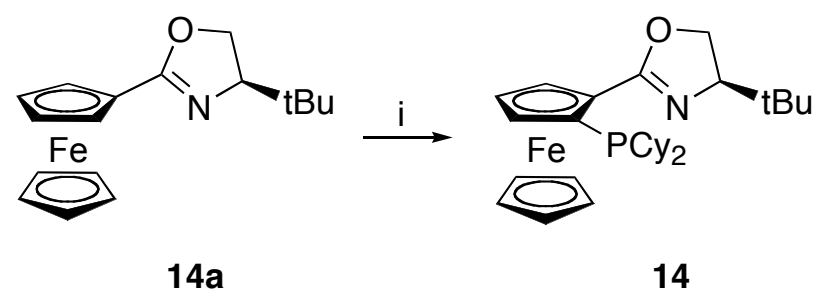

Reagents and conditions: Compound 14a was prepared according to the reported procedure. i. $n \mathrm{BuLi}$, THF, room temperature, $2 \mathrm{~h}, \mathrm{ClPCy}_{2}$, overnight, RT.

Preparation of Ligand 14. A $2.5 \mathrm{M}$ solution of $n \mathrm{BuLi}(80 \mu \mathrm{L}, 0.20 \mathrm{mmol})$ was added dropwise to the solution of $14 \mathbf{a}(62.2 \mathrm{mg}, 0.200 \mathrm{mmol})$ in $1.0 \mathrm{~mL}$ of $\mathrm{Et}_{2} \mathrm{O}$ at room temperature. The resulting mixture was stirred for $2 \mathrm{~h}$ at room temperature. A solution of $\mathrm{ClPCy}_{2}(46.5 \mathrm{mg}, 0.200 \mathrm{mmol})$ in $2.0 \mathrm{~mL}$ of $\mathrm{Et}_{2} \mathrm{O}$ was added through syringe. The mixture was stirred overnight. Aqueous saturated $\mathrm{NaHCO}_{3}(10$ $\mathrm{mL}$ ) was slowly added with cooling in an ice bath. The resulting organic layer and $\mathrm{Et}_{2} \mathrm{O}$ extracts from the aqueous layer were combined, washed with $2 \times 20 \mathrm{~mL}$ of water, dried over $\mathrm{MgSO}_{4}$, and concentrated to afford an organic powder. The crude product was purified by flash chromatography (hexane/ethyl acetate, $95 / 5)$ to give $89.3 \mathrm{mg}(88 \%)$ of the product as an orange solid. ${ }^{1} \mathrm{H}$ NMR $\left(\mathrm{C}_{6} \mathrm{D}_{6}\right)$

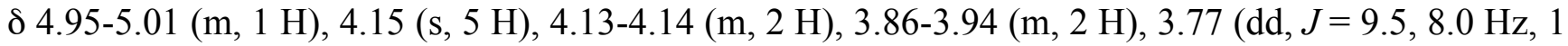
$\mathrm{H}), 2.58-2.61(\mathrm{~m}, 1 \mathrm{H}), 1.00-2.00(\mathrm{~m}, 21 \mathrm{H}), 0.95(\mathrm{~s}, 9 \mathrm{H}) ;{ }^{31} \mathrm{P}\left\{{ }^{1} \mathrm{H}\right\}$ NMR $\left(\mathrm{C}_{6} \mathrm{D}_{6}\right) \delta-10.14(\mathrm{~s}) .{ }^{13} \mathrm{C}$ $\operatorname{NMR}\left(\mathrm{C}_{6} \mathrm{D}_{6}\right) \delta 165.12(\mathrm{~d}, J=1.5 \mathrm{~Hz}), 80.82(\mathrm{~d}, J=32.2 \mathrm{~Hz}), 76.88,75.23(\mathrm{~d}, J=15.5 \mathrm{~Hz}), 72.23$, $72.19,71.26$ (d, $J=17.5 \mathrm{~Hz}), 71.20,70.34,68.00,37.86(\mathrm{~d}, J=19.2 \mathrm{~Hz}), 33.92(\mathrm{~d}, J=16.0 \mathrm{~Hz})$, 33.72, 32.48 (d, $J=19.7 \mathrm{~Hz}), 31.02(\mathrm{~d}, J=12.8 \mathrm{~Hz}), 30.40(\mathrm{~d}, J=13.1 \mathrm{~Hz}), 29.88(\mathrm{~d}, J=9.4 \mathrm{~Hz})$, $28.55(\mathrm{~d}, J=9.7 \mathrm{~Hz}), 28.32(\mathrm{~d}, J=8.4 \mathrm{~Hz}), 27.56(\mathrm{~d}, J=10.8 \mathrm{~Hz}), 27.42(\mathrm{~d}, J=9.7 \mathrm{~Hz}), 26.97(\mathrm{~d}, J=$ 8.7 Hz), 26.25. Anal. Calcd. For $\mathrm{C}_{29} \mathrm{H}_{42}$ FeNOP: C, 68.64; H, 8.34. Found: C, 68.55; H, 8.13.

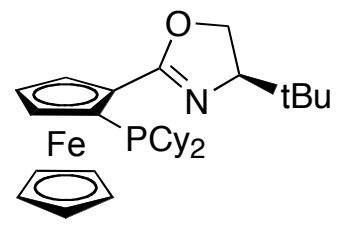

14<smiles>CC(C)CC(O)c1ccccc1Br</smiles>

$15 a$<smiles>CC([O-])c1ccccc1Br</smiles>

15b<smiles>CC(C)C(C)c1ccccc1Br</smiles>

$15 \mathrm{c}$

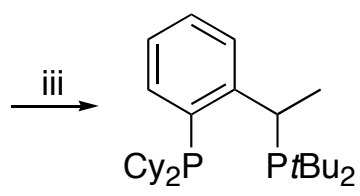

15

Reagents and conditions: i. a) $\mathrm{NaH}, \mathrm{Et}_{2} \mathrm{O}$, reflux, $5 \mathrm{~h}$; b) $\mathrm{TsCl}, 0^{\circ} \mathrm{C}, 1 \mathrm{~h}$; ii. a) $\mathrm{LiP} t \mathrm{~B} \mathrm{u}_{2} \bullet \mathrm{BH}_{3}$, $78^{\circ} \mathrm{C} \rightarrow$ reflux, $10 \mathrm{~h}$; iii. a) $t \mathrm{BuLi}, \mathrm{THF},{ }_{-} 78^{\circ} \mathrm{C}, 35 \mathrm{~min}, \mathrm{ClPCy}{ }_{2}$, overnight, $\mathrm{RT}$; b) Morpholine, $100{ }^{\circ} \mathrm{C}$, $1 \mathrm{~h}$.

Preparatiion of Ligand 15. A solution of 2-bromo- $\alpha$-methylbenzylaocohol 15a $(0.400 \mathrm{~g}, 2.00 \mathrm{mmol})$ in $2.0 \mathrm{~mL}$ of $\mathrm{Et}_{2} \mathrm{O}$ was added dropwise to a suspension of $\mathrm{NaH}(50.0 \mathrm{mg}, 2.00 \mathrm{mmol})$ in $3.0 \mathrm{~mL}$ of $\mathrm{Et}_{2} \mathrm{O}$ in a schlenk's tube nunder nitrogen atmosphere. The resulting mixture was stirred for $30 \mathrm{~min}$ at room temperature and heated to reflux for $5 \mathrm{~h}$. The mixture was cooled to $0{ }^{\circ} \mathrm{C}$ and tosyl chloride $(1.9$ 
$\mathrm{mmol}$ ) in $5.0 \mathrm{~mL} \mathrm{Et}{ }_{2} \mathrm{O}$ was added dropwise. The mixture was stirred for another hour at $0{ }^{\circ} \mathrm{C}$. The mixture was filtered through a short-pass funnel with Celite. The solvent was evaporated under reduced pressure. Recrystallizition from $\mathrm{Et}_{2} \mathrm{O} /$ hexane at $-10{ }^{\circ} \mathrm{C}$ gave $675 \mathrm{mg}(95 \%)$ of compound $\mathbf{1 5 b}$ as white crystals. ${ }^{1} \mathrm{H}$ NMR $\left(\mathrm{CDCl}_{3}\right) \delta 7.65(\mathrm{~d}, J=8.5 \mathrm{~Hz}, 2 \mathrm{H}), 7.34(\mathrm{ddd}, J=8.0,5.5,1.0 \mathrm{~Hz}, 2 \mathrm{~h})$, 7.14-7.18 (m, $3 \mathrm{H}), 7.03(\mathrm{td}, J=8.0,1.5 \mathrm{~Hz}, 1 \mathrm{H}), 5.89(\mathrm{t}, J=6.5 \mathrm{~Hz}, 1 \mathrm{H}), 2.32(\mathrm{~s}, 3 \mathrm{H}), 1.50(\mathrm{~d}, J=$ $6.5 \mathrm{~Hz}, 3 \mathrm{H}) ;{ }^{13} \mathrm{C}$ NMR $\left(\mathrm{CDCl}_{3}\right) \delta 144.49,139.16,133.44,132.32,129.47,129.36,127.72,127.66$, 127.35, 120.82, 79.09, 22.80, 21.40. Anal. Calcd. For $\mathrm{C}_{15} \mathrm{H}_{15} \mathrm{BrO}_{3} \mathrm{~S}: \mathrm{C}, 50.71 ; \mathrm{H}, 4.26$. Found: $\mathrm{C}$, $50.67 ; \mathrm{H}, 4.23$.

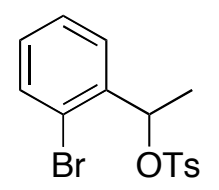

15b

A $1.0 \mathrm{M}$ solution of $n \mathrm{BuLi}(0.4 \mathrm{~mL}, 1.0 \mathrm{mmol})$ was added dropwise to a solution of $\mathrm{HPt} \mathrm{Bu}_{2} \bullet \mathrm{BH}_{3}$ $(0.160 \mathrm{~g}, 1.00 \mathrm{mmol})$ in $2.0 \mathrm{~mL}$ of THF at $-78^{\circ} \mathrm{C}$. The resulting mixture was stirred for $0.5 \mathrm{~h}$ at $-78^{\circ} \mathrm{C}$ and $0.5 \mathrm{~h}$ at room temperature. The resulting solution was then added to a solution of $\mathbf{1 5 b}(0.354 \mathrm{~g}$, $1.00 \mathrm{mmol}$ ) in $3.0 \mathrm{~mL}$ of THF at $-78^{\circ} \mathrm{C}$. The mixture was stirred for $0.5 \mathrm{~h}$ at room temperature and heated to reflux for $10 \mathrm{~h}$. White precipitate was formed during this time. After cooling to room temperature, the mixture was filtered through a short-pass funnel containing Celite. The solvent was evaporated under reduced pressure. The residue was purified by flash chromatography (hexane/ethyl acetate, 99/1) to give $295.3 \mathrm{mg}(92 \%)$ of $\mathbf{1 5 c}$ as a white solid. ${ }^{1} \mathrm{H}$ NMR $\left(\mathrm{CDCl}_{3}\right) \delta 7.85(\mathrm{~d}, J=8.4 \mathrm{~Hz}$, $1 \mathrm{H}), 7.43(\mathrm{~d}, J=8.0 \mathrm{~Hz}, 1 \mathrm{H}), 7.18-7.22(\mathrm{~m}, 1 \mathrm{H}), 6.97-7.01(\mathrm{~m}, 1 \mathrm{H}), 4.06(\mathrm{dq}, J=14.4,7.2 \mathrm{~Hz}, 1$ H), 1.52 (dd, $J=13.2,7.2 \mathrm{~Hz}, 3 \mathrm{H}), 1.37$ (d, $J=12.0 \mathrm{~Hz}, 9 \mathrm{H}), 1.01$ (d, $J=12.0 \mathrm{~Hz}, 9 \mathrm{H}), 0.10-1.00$ $(\mathrm{m}, 3 \mathrm{H}) ;{ }^{13} \mathrm{C}$ NMR $\left(\mathrm{CDCl}_{3}\right) \delta 142.80,132.67(\mathrm{~d}, J=2.7 \mathrm{~Hz}), 131.82(\mathrm{~d}, J=2.2 \mathrm{~Hz}), 128.33(\mathrm{~d}, J=$ $1.5 \mathrm{~Hz}), 127.58$ (d, $J=1.7 \mathrm{~Hz}), 124.03(\mathrm{~d}, J=6.2 \mathrm{~Hz}), 34.49$ (d, $J=23.6 \mathrm{~Hz}), 34.17$ (d, $J=24.5 \mathrm{~Hz})$, $31.98(\mathrm{~d}, J=22.5 \mathrm{~Hz}), 29.22,29.14,22.55(\mathrm{~d}, J=1.3 \mathrm{~Hz}) .{ }^{31} \mathrm{P}\left\{{ }^{1} \mathrm{H}\right\} \mathrm{NMR}\left(\mathrm{CDCl}_{3}\right) \delta 58.5(\mathrm{~m})$.

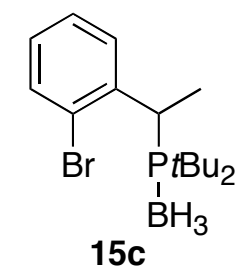

A $1.5 \mathrm{M}$ solution of $t \mathrm{BuLi}(0.54 \mathrm{~mL}, 0.81 \mathrm{mmol})$ was added dropwise to the solution of $\mathbf{1 5 c}(0.278 \mathrm{~g}$, $0.810 \mathrm{mmol}$ ) in $2.0 \mathrm{~mL}$ of THF at $-78^{\circ} \mathrm{C}$. The resulting mixture was stirred for $1 \mathrm{~h}$ at $-78^{\circ} \mathrm{C}$. To this yellow solution, $\mathrm{ClPCy}_{2}(0.189 \mathrm{~g}, 0.900 \mathrm{mmol})$ was added. The resulting mixture was stirred at $-78^{\circ} \mathrm{C}$ for $2 \mathrm{~h}$ and then heated to $60^{\circ} \mathrm{C}$ for $5 \mathrm{~h}$. The solution was cooled to room temperature, and $3.0 \mathrm{~mL}$ of $\mathrm{BH}_{3} \bullet \mathrm{THF}(1.0 \mathrm{M})$ was added. The resulting solution was stirred for $10 \mathrm{~min}$, and $5.0 \mathrm{~mL}$ of $\mathrm{MeOH}$ was added slowly with cooling in an ice bath to quench the excess $\mathrm{BH}_{3}$. The solvent was removed under reduced pressure, and the residue was purified by flash chromatography (hexane/ethyl acetate, 90/00) to give $203.3 \mathrm{mg}(53 \%)$ of $\mathbf{1 5} \cdot \mathrm{BH}_{3}$ as a white solid. ${ }^{1} \mathrm{H}$ NMR $\left(\mathrm{CDCl}_{3}\right) \delta 8.22(\mathrm{dd}, J=7.6,4.4 \mathrm{~Hz}, 1$ $\mathrm{H}), 7.45(\mathrm{t}, J=8.0 \mathrm{~Hz}, 1 \mathrm{H}), 7.38(\mathrm{t}, J=8.0 \mathrm{~Hz}, 1 \mathrm{H}), 7.23(\mathrm{t}, J=7.6 \mathrm{~Hz}, 1 \mathrm{H}), 4.89$ (dq, $J=14.4,6.8$ $\mathrm{Hz}, 1 \mathrm{H}), 2.18-2.30(\mathrm{~m}, 1 \mathrm{H}), 1.71-1.98(\mathrm{~m}, 9 \mathrm{H}), 1.58(\mathrm{dd}, J=13.2,7.2 \mathrm{~Hz}, 3 \mathrm{H}), 1.40-1.60(\mathrm{~m}, 3 \mathrm{H})$, $1.41(\mathrm{~d}, J=12.0 \mathrm{~Hz}, 9 \mathrm{H}), 1.00-1.26(\mathrm{~m}, 9 \mathrm{H}), 1.00(\mathrm{~d}, J=12.0 \mathrm{~Hz}, 9 \mathrm{H}), 0.00-1.00(\mathrm{~m}, 6 \mathrm{H}) ;{ }^{31} \mathrm{P}\left\{{ }^{1} \mathrm{H}\right\}$ NMR $\left(\mathrm{CDCl}_{3}\right) \delta 24.79(\mathrm{~m}), 58.75(\mathrm{~m})$. 


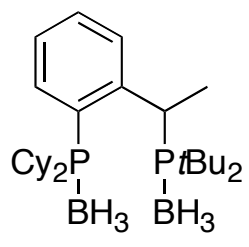

$15 \cdot 2 \mathrm{BH}_{3}$

A colorless solution of $\mathbf{1 5} \cdot 2 \mathrm{BH}_{3}(100.0 \mathrm{mg}, 0.224 \mathrm{mmol})$ in $1.0 \mathrm{~mL}$ of morpholine was heated at 100 ${ }^{\circ} \mathrm{C}$ for $1 \mathrm{~h}$. The solvent was evaporated, and $5.0 \mathrm{~mL}$ of hexane was added. The solution was filtered through a short plug of Celite, and the solvent was evaporated under reduced pressure. Recrystallization of the residue from $3.0 \mathrm{~mL}$ of degassed $\mathrm{EtOH}$ yielded $85.0 \mathrm{mg}(85 \%)$ of ligand 15 as white crystals. ${ }^{1} \mathrm{H}$ NMR $\left(\mathrm{C}_{6} \mathrm{D}_{6}\right) \delta$ 7.60-7.64 (m, $\left.1 \mathrm{H}\right), 7.07(\mathrm{~d}, J=8.0 \mathrm{~Hz}, 1 \mathrm{H}), 6.86-6.88(\mathrm{~m}, 1 \mathrm{H})$, $6.71(\mathrm{td}, J=7.5,1.5 \mathrm{~Hz}, 1 \mathrm{H}), 4.47-4.55(\mathrm{~m}, 1 \mathrm{H}), 1.68-1.78(\mathrm{~m}, 3 \mathrm{H}), 1.37-1.49(\mathrm{~m}, 6 \mathrm{H}), 1.32(\mathrm{dd}, J$ $=8.5,7.0 \mathrm{~Hz}, 3 \mathrm{H}), 1.17-1.26(\mathrm{~m}, 2 \mathrm{H}), 1.03(\mathrm{~d}, J=10.0 \mathrm{~Hz}, 9 \mathrm{H}), 1.00-1.26(\mathrm{~m}, 9 \mathrm{H}), 0.80-1.00(\mathrm{~m}, 2$ $\mathrm{H}), 0.89(\mathrm{~d}, J=10.0 \mathrm{~Hz}, 9 \mathrm{H}) ;{ }^{13} \mathrm{C} \mathrm{NMR}\left(\mathrm{C}_{6} \mathrm{D}_{6}\right) \delta 156.07(\mathrm{dd}, J=24.9,9.2 \mathrm{~Hz}), 133.25(\mathrm{~d}, J=17.5$ $\mathrm{Hz}), 133.12(\mathrm{~d}, J=2.8 \mathrm{~Hz}), 130.38(\mathrm{dd}, J=14.7,4.6 \mathrm{~Hz}), 128.74,124.99(\mathrm{~d}, J=1.8 \mathrm{~Hz}), 36.16(\mathrm{~d}, J=$ $15.6 \mathrm{~Hz}), 32.61-32.09$ (4 C), 31.97 (dd, $J=11.9,2.6 \mathrm{~Hz}), 31.83$ (d, $J=12.9 \mathrm{~Hz}), 31.40$ (d, $J=15.7$ $\mathrm{Hz}), 30.99(\mathrm{~d}, J=13.8 \mathrm{~Hz}), 30.71(\mathrm{~d}, J=17.5 \mathrm{~Hz}), 29.59(\mathrm{~d}, J=6.5 \mathrm{~Hz}), 27.77(\mathrm{~d}, J=11.9 \mathrm{~Hz}), 27.68$ $(\mathrm{d}, J=6.4 \mathrm{~Hz}), 27.52(\mathrm{~d}, J=10.2 \mathrm{~Hz}), 27.34(\mathrm{~d}, J=11.1 \mathrm{~Hz}), 26.64(\mathrm{~d}, J=26.77 \mathrm{~Hz}), 24.81(\mathrm{~d}, J=$ 12.8 Hz), 10.5. ${ }^{31} \mathrm{P}\left\{{ }^{1} \mathrm{H}\right\}$ NMR $\left(\mathrm{CDCl}_{3}\right) \delta-16.98(\mathrm{~s}), 53.59$ (s). Anal. Calcd. For $\mathrm{C}_{28} \mathrm{H}_{48} \mathrm{P}_{2}: \mathrm{C}, 75.30 ; \mathrm{H}$, 10.83. Found: C, 74.87; H, 11.27.

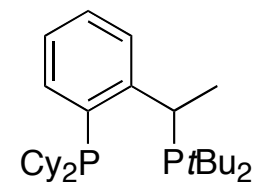

15

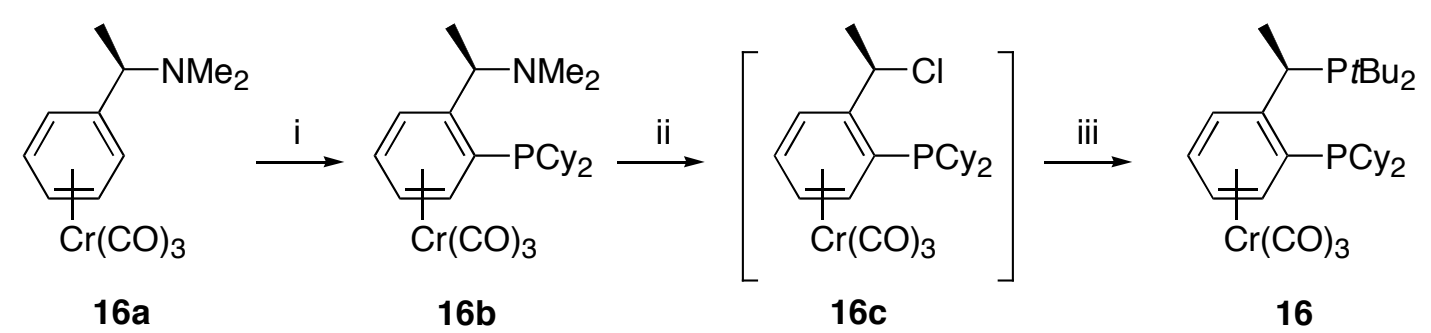

Reagents and conditions: i. $t \mathrm{BuLi}, \mathrm{THF}, 7^{\circ} \mathrm{C}, 1 \mathrm{~h}, \mathrm{ClPCy}_{2}$, overnight, RT; ii. 1-Chloroethyl chloroformate, $\mathrm{THF},-40{ }^{\circ} \mathrm{C} \rightarrow$ room temperature, overnight; iii. a) $\mathrm{HP} t \mathrm{Bu}_{2}, \mathrm{TlPF}_{6}$, acetone, room temperature, overnight; b) $\mathrm{Et}_{3} \mathrm{~N}$, room temperature, $15 \mathrm{~min}$.

Preparation of Ligand 16. A $1.5 \mathrm{M}$ solution of $t \mathrm{BuLi}(0.73 \mathrm{~mL}, 1.00 \mathrm{mmol})$ was added dropwise to the solution of $16 \mathbf{a}(0.285 \mathrm{~g}, 1.00 \mathrm{mmol})$ in $2.0 \mathrm{~mL}$ of $\mathrm{THF}$ at $-78^{\circ} \mathrm{C}$. The resulting mixture was stirred for $1 \mathrm{~h}$ at $-78{ }^{\circ} \mathrm{C}$. To this yellow solution, $\mathrm{ClPCy}_{2}(0.210 \mathrm{~g}, 1.00 \mathrm{mmol})$ was added. The resulting mixture was stirred at $-78{ }^{\circ} \mathrm{C}$ for $2 \mathrm{~h}$ and then room temperature overnight. Aqueous saturated $\mathrm{NaHCO}_{3}$ $(10 \mathrm{~mL})$ was slowly added with cooling in an ice bath. The resulting organic layer and $\mathrm{Et}_{2} \mathrm{O}$ extracts from the aqueous layer were combined, washed with $2 \times 20 \mathrm{~mL}$ of water, dried over $\mathrm{MgSO}_{4}$, and concentrated to afford a yellow powder. Compound $\mathbf{1 6 b}$ was purified by flash chromatography (hexane/diethyl ether, 80/20) to give $0.397 \mathrm{~g}(80 \%)$ of the product $\mathbf{1 6 b}$ as a yellow solid. ${ }^{1} \mathrm{H}$ NMR $\left(\mathrm{C}_{6} \mathrm{D}_{6}\right) \delta 5.03(\mathrm{~d}, J=5.5 \mathrm{~Hz}, 1 \mathrm{H}), 4.63(\mathrm{t}, J=6.0 \mathrm{~Hz}, 1 \mathrm{H}), 4.41-4.48(\mathrm{~m}, 3 \mathrm{H}), 2.52-2.55(\mathrm{~m}, 1 \mathrm{H})$, 
$1.93(\mathrm{~s}, 6 \mathrm{H}), 1.36-1.87(\mathrm{~m}, 14 \mathrm{H}), 1.01-1.25(\mathrm{~m}, 7 \mathrm{H}), 0.83(\mathrm{~d}, J=7.0 \mathrm{~Hz}, 3 \mathrm{H}) ;{ }^{31} \mathrm{P}\left\{{ }^{1} \mathrm{H}\right\} \mathrm{NMR}$ $\left(\mathrm{C}_{6} \mathrm{D}_{6}\right) \delta-6.52 \mathrm{ppm}$.

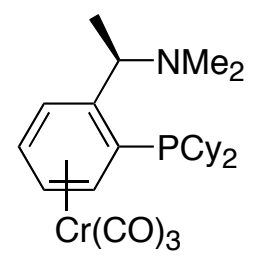

$16 b$

1-Chloroethyl chloroformate $(0.143 \mathrm{~g}, 1.00 \mathrm{mmol})$ was added dropwise to a stirring solution of $\mathbf{1 6 b}$ $(0.100 \mathrm{~g}, 0.250 \mathrm{mmol})$ in $5.0 \mathrm{~mL}$ of $\mathrm{THF}$ at $-40^{\circ} \mathrm{C}$. The solution was warmed to room temperature and kept stirring overnight. The solvent was evaporated and the residue was dissolved in $5.0 \mathrm{~mL}$ of $\mathrm{Et}_{2} \mathrm{O}$. The solution was filtered through a short pad of Celite, and the solvent was evaporated under reduced pressure to give a yellow solid. Attempts at purification by flash chromatography resulted in decomposition. The crude material was used without further purification.

$\mathrm{HPt} \mathrm{Bu}_{2}(36.6 \mathrm{mg}, 0.250 \mathrm{mmol})$ was added to the solution of the crude product dissolved in $5.0 \mathrm{~mL}$ of dry acetone. To this solution was added dropwise a suspension of $\mathrm{TlPF}_{6}(88.0 \mathrm{mg}, 0.250 \mathrm{mmol})$ in 1.0 $\mathrm{mL}$ of dry acetone. A fine white formed immediately. The solution was stirred at room temperature overnight. $\mathrm{NEt}_{3}(0.5 \mathrm{~mL})$ was added, and the solution was further stirred for $15 \mathrm{~min}$ and then filtered through a short plug of Celite. The solvent and excess $\mathrm{NEt}_{3}$ were evaporated under reduced pressure. The residue was purified by flash chromatography (hexane/ $\left.\mathrm{Et}_{2} \mathrm{O}, 95 / 5\right)$ to give $104.5 \mathrm{mg}(70 \%)$ of ligand 16 as a yellow solid. ${ }^{1} \mathrm{H}$ NMR $\left(\mathrm{C}_{6} \mathrm{D}_{6}\right) \delta 5.12(\mathrm{~d}, J=6.5 \mathrm{~Hz}, 1 \mathrm{H}), 4.67-4.71(\mathrm{~m}, 2 \mathrm{H}), 4.35(\mathrm{td}, J$ $=6.0,2.0 \mathrm{~Hz}, 1 \mathrm{H}), 4.12$ (quintet, $J=6.5 \mathrm{~Hz}, 1 \mathrm{H}), 1.53(\mathrm{dd}, J=7.5,2.5 \mathrm{~Hz}, 3 \mathrm{H}), 1.1-2.5(\mathrm{~m}, 22 \mathrm{H})$, $1.30(\mathrm{~d}, J=11.0 \mathrm{~Hz}, 9 \mathrm{H}), 1.02(\mathrm{~d}, J=11.0 \mathrm{~Hz}, 9 \mathrm{H}) ;{ }^{31} \mathrm{P}\left\{{ }^{1} \mathrm{H}\right\} \operatorname{NMR}\left(\mathrm{C}_{6} \mathrm{D}_{6}\right) \delta 50.23(\mathrm{~d}, J=52.67 \mathrm{~Hz})$, -13.66 (d, $J=52.67 \mathrm{~Hz})$ ppm. ${ }^{13} \mathrm{C}$ NMR $\left(\mathrm{C}_{6} \mathrm{D}_{6}\right) \delta 233.79,125.62(\mathrm{dd}, \mathrm{J}=20.5,16.5 \mathrm{~Hz}), 107.57(\mathrm{~d}, J$ $=38.5 \mathrm{~Hz}), 98.18(\mathrm{~d}, J=2.1 \mathrm{~Hz}), 94.18,90.40(\mathrm{t}, J=5.5 \mathrm{~Hz}), 89.03,38.30(\mathrm{dd}, \mathrm{J}=13.8,9.6 \mathrm{~Hz})$, $36.46(\mathrm{dd}, \mathrm{J}=19.2,4.5 \mathrm{~Hz}), 35.25(\mathrm{~d}, \mathrm{~J}=16.7 \mathrm{~Hz}), 35.14(\mathrm{~d}, \mathrm{~J}=33.1 \mathrm{~Hz}), 34.93(\mathrm{~d}, \mathrm{~J}=16.9 \mathrm{~Hz})$, $34.63(\mathrm{~d}, \mathrm{~J}=32.1 \mathrm{~Hz}), 33.11(\mathrm{~d}, \mathrm{~J}=23.5 \mathrm{~Hz}), 32.55(\mathrm{dd}, \mathrm{J}=15.6,7.3 \mathrm{~Hz}), 32.42(\mathrm{~d}, \mathrm{~J}=13.5 \mathrm{~Hz})$, $31.74(\mathrm{dd}, \mathrm{J}=12.5,2.9 \mathrm{~Hz}), 30.03(\mathrm{~d}, \mathrm{~J}=12.2 \mathrm{~Hz}), 28.81(\mathrm{~d}, \mathrm{~J}=9.3 \mathrm{~Hz}), 28.68$ (d, J = 12.8 Hz), 28.01 $(\mathrm{d}, \mathrm{J}=7.3 \mathrm{~Hz}), 27.45(\mathrm{~d}, \mathrm{~J}=6.9 \mathrm{~Hz}), 27.41(\mathrm{~d}, \mathrm{~J}=13.7 \mathrm{~Hz}), 26.89,26.72$ (d, J = 1.6 Hz). Anal. Calcd. For $\mathrm{C}_{32} \mathrm{H}_{51} \mathrm{CrO}_{3} \mathrm{P}_{2}$ : C, 64.30; H, 8.60. Found: C, 63.87; H, 9.05.

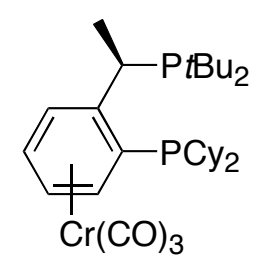

16

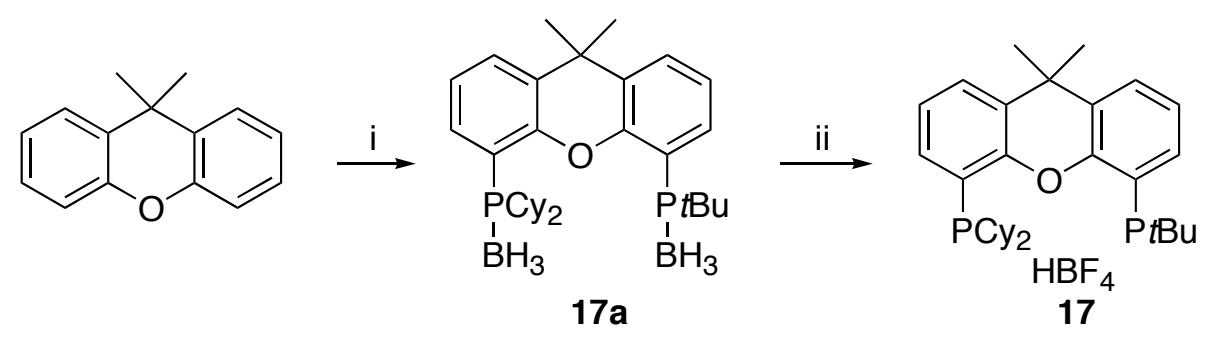


Reagents and conditions: i. a) BuLi/TMEDA, $\mathrm{Et}_{2} \mathrm{O},-78{ }^{\circ} \mathrm{C} \rightarrow$ room temperature for $16 \mathrm{~h}$; b) $\mathrm{ClP} t \mathrm{Bu}_{2},-78{ }^{\circ} \mathrm{C} \rightarrow$ room temperature, $16 \mathrm{~h}$; c) $\mathrm{BuLi} / \mathrm{TMEDA}, \mathrm{Et}_{2} \mathrm{O},-78{ }^{\circ} \mathrm{C} \rightarrow$ room temperature for 16 $\mathrm{h}$; d) $\mathrm{ClPCy}_{2},-78^{\circ} \mathrm{C} \rightarrow$ room temperature, $16 \mathrm{~h}$; e) $\mathrm{BH}_{3} / \mathrm{THF}$, room temperature, $10 \mathrm{~min}$; ii. a) TMEDA, reflux, $10 \mathrm{~h}$; b) $\mathrm{HBF}_{4} / \mathrm{CH}_{2} \mathrm{Cl}_{2}$, room temperature, $30 \mathrm{~min}$.

Preparatiion of Ligand 17. A solution (1.40 M) of sec-Butyllithium (1.50 mL, $2.10 \mathrm{mmol})$ was added dropwise to a mixture of Xanthene $(0.420 \mathrm{~g}, 2.0 \mathrm{mmol})$ and TMEDA $(0.244 \mathrm{~g}, 2.10 \mathrm{mmol})$ in $\mathrm{Et}_{2} \mathrm{O}$ $(5.0 \mathrm{~mL})$. The resulting mixture was stirred for $16 \mathrm{~h}$ at room temperature. The mixture was cooled to $78{ }^{\circ} \mathrm{C}$, and di-tert-butyl-chlorophosphine $(0.361 \mathrm{~g}, 2.00 \mathrm{mmol})$ in $5.0 \mathrm{~mL} \mathrm{Et}_{2} \mathrm{O}$ was added dropwise. The mixture was stirred for $16 \mathrm{~h}$ at room temeperature before it was cooled to $-78{ }^{\circ} \mathrm{C}$. TMEDA $(0.244$ $\mathrm{g}, 2.10 \mathrm{mmol})$ was added, followed by a solution $(1.40 \mathrm{M})$ of $\mathrm{sec}$-butyllithium $(1.50 \mathrm{~mL}, 2.10 \mathrm{mmol})$. The resulting mixture was stirred for $16 \mathrm{~h}$ at room temperature. The mixture was cooled to $-78{ }^{\circ} \mathrm{C}$ and di-cyclohexyl-chlorophosphine $(0.465 \mathrm{~g}, 2.00 \mathrm{mmol})$ in $5.0 \mathrm{~mL} \mathrm{Et}_{2} \mathrm{O}$ was added dropwise. The mixture was stirred for $16 \mathrm{~h}$ at room temeperature. $\mathrm{BH}_{3}$ in THF $(30.0 \mathrm{~mL}, 1.0 \mathrm{M})$ was added, and the mixture was stirred for another $2 \mathrm{~h}$ at room temperature. The excess $\mathrm{BH}_{3}$ was quenched by methanol. The solvent was evaporated under reduced pressure, and the residue was purified by flash chromatography (hexane/ethyl acetate, 90/10) to give a white solid. The white solid was further purified by recrystallization from ethyl acetate to give 17 a $(300.9 \mathrm{mg}, 26 \%)$ as colorless crystals. ${ }^{1} \mathrm{H}$ NMR $\left(\mathrm{CDCl}_{3}\right) \delta 7.91(\mathrm{ddd}, J=13.2,7.2,1.2 \mathrm{~Hz}, 1 \mathrm{H}), 7.63(\mathrm{dt}, J=7.6,1.6 \mathrm{~Hz}, 1 \mathrm{H}), 7.54(\mathrm{~d}, J=7.2 \mathrm{~Hz}, 1$ H), 7.46 (dd, $J=7.6,1.6 \mathrm{~Hz} 1 \mathrm{H}), 7.10-7.14(\mathrm{~m}, 2 \mathrm{H}), 3.60-3.64(\mathrm{~m}, 2 \mathrm{H}), 2.13$ (d, $J=11.6 \mathrm{~Hz}, 2 \mathrm{H})$, 1.73-1.82 (m, $4 \mathrm{H}), 1.57$ (s, $6 \mathrm{H}), 1.26$ (d, $J=12.0 \mathrm{~Hz}, 18 \mathrm{H}), 1.10-1.62$ (m, $14 \mathrm{H}), 0.01-1.00(\mathrm{~m}, 6 \mathrm{H})$; ${ }^{31} \mathrm{P}\left\{{ }^{1} \mathrm{H}\right\} \mathrm{NMR}\left(\mathrm{CDCl}_{3}\right) \delta 38.4$ (br), 10.54 (br).

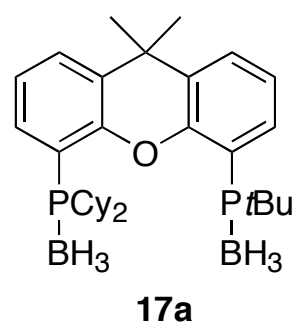

A colorless solution of $\mathbf{1 7 a}(100.0 \mathrm{mg}, 0.173 \mathrm{mmol})$ in $10.0 \mathrm{~mL}$ of TMEDA was heated at $110^{\circ} \mathrm{C}$ for 5 $\mathrm{h}$. The solvent was removed, and $5.0 \mathrm{~mL}$ of hexane was added. The solution was filtered through a short plug of Celite, and the solvent was evaporated under reduced pressure. The residue was dissolved in $3.0 \mathrm{~mL}$ of $\mathrm{CH}_{2} \mathrm{Cl}_{2}$, and aqueous $\mathrm{HBF}_{4}(43 \% \mathrm{w} / \mathrm{w}, 0.2 \mathrm{~mL})$ was added. After vigiously stirring for 10 min, the organic layer was separated and dried over $\mathrm{MgSO}_{4}$. Filtration, followed by removal of the solvent and recrystallization of the residue form $\mathrm{CH}_{2} \mathrm{Cl}_{2}$ by the addition of pentane afforded the product as colorless crystals. ${ }^{1} \mathrm{H} \mathrm{NMR}\left(\mathrm{CD}_{2} \mathrm{Cl}_{2}\right) \delta 8.48(\mathrm{dd}, J=478.3,7.2 \mathrm{~Hz}, 1 \mathrm{H}), 7.91(\mathrm{~d}, J=8.0 \mathrm{~Hz}, 1 \mathrm{H})$, $7.65(\mathrm{t}, J=8.4 \mathrm{~Hz}, 1 \mathrm{H}), 7.48-7.54(\mathrm{~m}, 2 \mathrm{H}), 7.38(\mathrm{~d}, J=7.6 \mathrm{~Hz}, 1 \mathrm{H}), 7.27(\mathrm{t}, J=7.6 \mathrm{~Hz}, 1 \mathrm{H}), 2.06(\mathrm{t}$, $J=7.2 \mathrm{~Hz}, 2 \mathrm{H}), 1.95(\mathrm{~d}, J=7.5 \mathrm{~Hz}, 2 \mathrm{H}), 1.79$ (d, $J=12.8 \mathrm{~Hz}, 2 \mathrm{H}), 1.65(\mathrm{~s}, 6 \mathrm{H}), 1.57$ (d, $J=13.2$ $\mathrm{Hz}, 18 \mathrm{H}), 1.50-1.70(\mathrm{~m}, 5 \mathrm{H}), 0.95-1.42(\mathrm{~m}, 11 \mathrm{H}) ;{ }^{31} \mathrm{P}\left\{{ }^{1} \mathrm{H}\right\} \mathrm{NMR}\left(\mathrm{CD}_{2} \mathrm{Cl}_{2}\right) \delta 22.0(\mathrm{~d}, J=89.0 \mathrm{~Hz})$, $16.55(\mathrm{~d}, J=89.0 \mathrm{~Hz}) .{ }^{13} \mathrm{C} \mathrm{NMR}\left(\mathrm{CD}_{2} \mathrm{Cl}_{2}\right) \delta 154.60(\mathrm{~d}, J=3.0 \mathrm{~Hz}), 154.30(\mathrm{~d}, J=18.4 \mathrm{~Hz}), 134.76(\mathrm{~d}$, $J=4.5 \mathrm{~Hz}), 133.14,132.41(\mathrm{~d}, J=3.0 \mathrm{~Hz}), 131.37,130.73(\mathrm{~d}, J=5.3 \mathrm{~Hz}), 126.95,125.63(\mathrm{~d}, J=11.4$ $\mathrm{Hz}), 124.94,122.19$ (d, $J=19.1 \mathrm{~Hz}), 103.52$ (d, $J=70.4 \mathrm{~Hz}), 35.81,35.13$ (d, J = 33.5 Hz), 33.24 (d, J $=9.9 \mathrm{~Hz}), 30.98(\mathrm{~d}, J=16.0 \mathrm{~Hz}), 30.01,29.53(\mathrm{~d}, J=6.8 \mathrm{~Hz}), 27.93,27.29(\mathrm{~d}, J=13.0 \mathrm{~Hz}), 27.11(\mathrm{~d}$, $J=8.3 \mathrm{~Hz}), 26.53$. 


\section{References:}

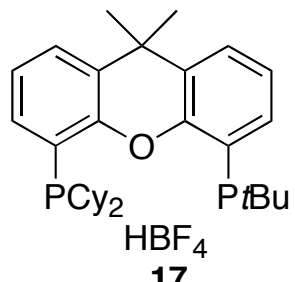

1. Wagaw, S.; Buchwald, S. L. J. Org. Chem. 1996, 61, 7240-7241.

2. Wolfe, J. P.; Tomori, H.; Sadighi, J. P.; Yin, J.; Buchwald, S. L. J. Org. Chem. 2000, 65, 11581174.

3. Morris, J.; Wishka, D. J. J. Org. Chem. 1995, 60, 2642 - 2644.

4. Noveron, J. C.; Lah, M. S.; Del Sesto, R. E.; Arif, A. M.; Miller, J. S.; Stang, P. J. J. Am. Chem. Soc. 2002, 124, 6613-6625.

5. Zhang, Z.; Mao, J.; Zhu, D.; Wu, F.; Chen, H.; Wan, B. Tetrahedron, 2006, 62, 4435.

6. Ma, D. W.; Cai, Q.; Zhang, H. Org. Lett. 2003, 5, 2453 - 2455.

7. Brown, D. S.; Elliott, M. C.; Moody, C. J.; Mowlem, T. J.; Marino, J. P. Jr; Padwa, A. J. Org. Chem. 1994, 59, 2447-2455.

8. Urgaonkar, S.; Nagarajum, M.; Verkade, J. G. Org. Lett. 2003, 5, 815-818.

9. Grail, G. F.; Renenbaum, L. E.; Tolstoouhov, A. V.; Duca, C. J.; Reinhard, J. F.; Anderson, F. E.; Scudi, J. V. J. Am. Chem. Soc. 1952, 74, 1313.

10. Cho, C. S.; Kim, H. S.; Kim, T. J.; Shim, S. C. Synth. Commun. 2001, 31, 3791.

11. Urgaonkar, S.; Verkade, J. G. J. Org. Chem. 2004, 69, 9135.

12. Clark, V. M.; Cox, A.; Herbert, E. J. J. Chem. Soc. 1968, 831-833.

13. Zhou, C.; Wang, Y.; Li, D.; Zhou, L.; Liu, P.; Shi, Q. Eur. J. Inorg. Chem. 2006, 2437-2446.

14. Tundel, R. E.; Anderson, K. W.; Buchwald, S. L. J. Org. Chem. 2006, 71, 430-433.

15. Dai, Q.; Gao, W.; Liu, D.; Kapes, L. M.; Zhang, X. J. Org. Chem. 2006, 71, 3928-3934.

16. Grasa, G. A.; Viciu, M. S.; Huang, J.; Nolan, S. P. J. Org. Chem. 2001, 66, 7729-7737.

17. Zhang, H.; Cai, Q,; Ma, D. J. Org. Chem. 2005, 70, 5164-5173.

18. Xu, C.; Gong, J.; Wu, Y. Tetrahedron Lett. 2007, 48, 1619-1623.

19. Xie, X.; Zhang, T. Y.; Zhang, Z. J. Org. Chem. 2006, 71, 6522-6529.

20. Noriyasu, K.; Quinetta, S.; Stambuli, J. P.; Hartwig, J. F. J. Org. Chem. 2002, 67, 5553-5566.

21. Ryoichi, H.; Masahiro, K.; Tetsutaro, K.; Kiyoshi, T.; Fumio, Y. J. Heterocyclic Chem. 1989, 26, 1255-1259. 


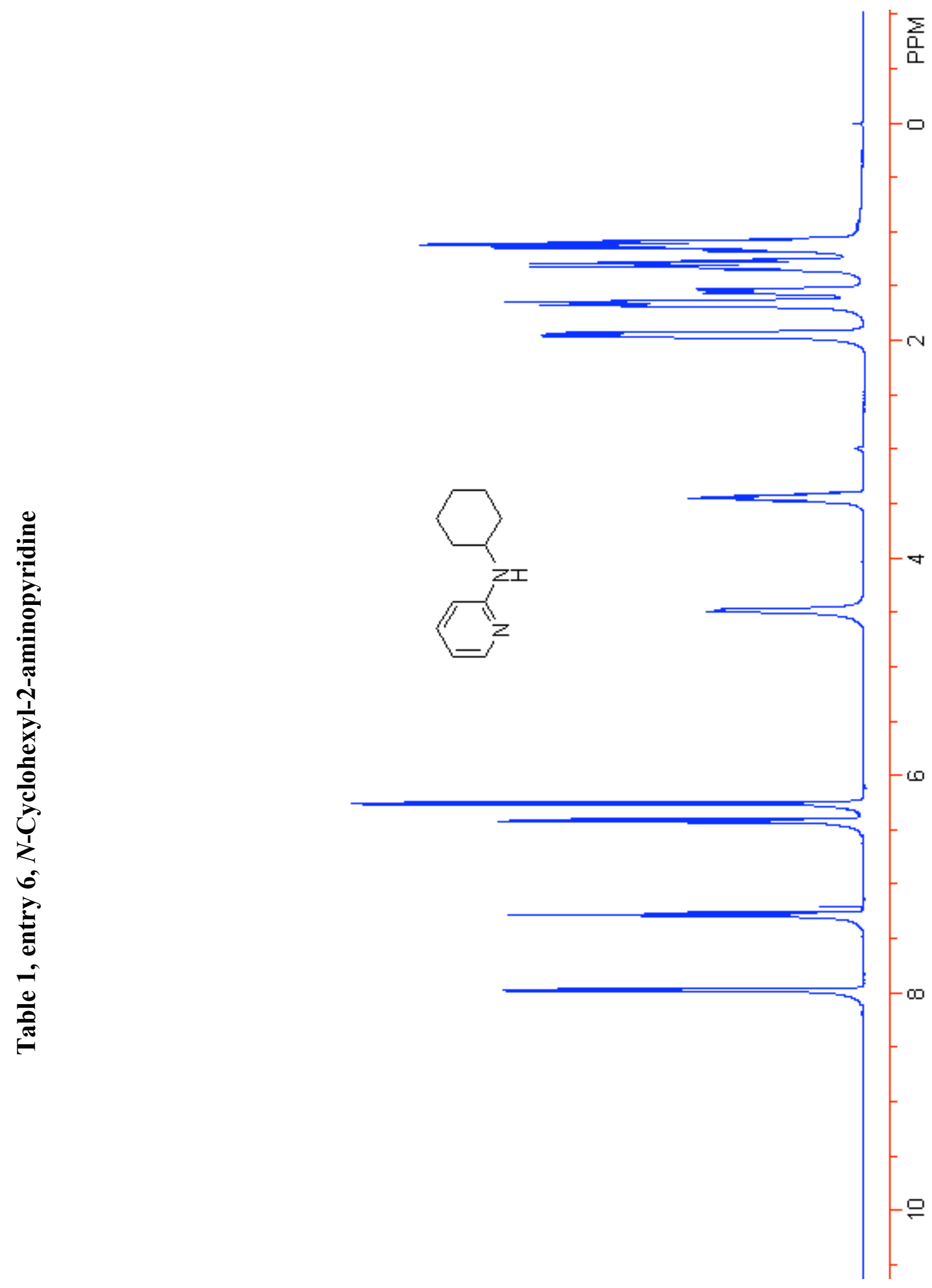



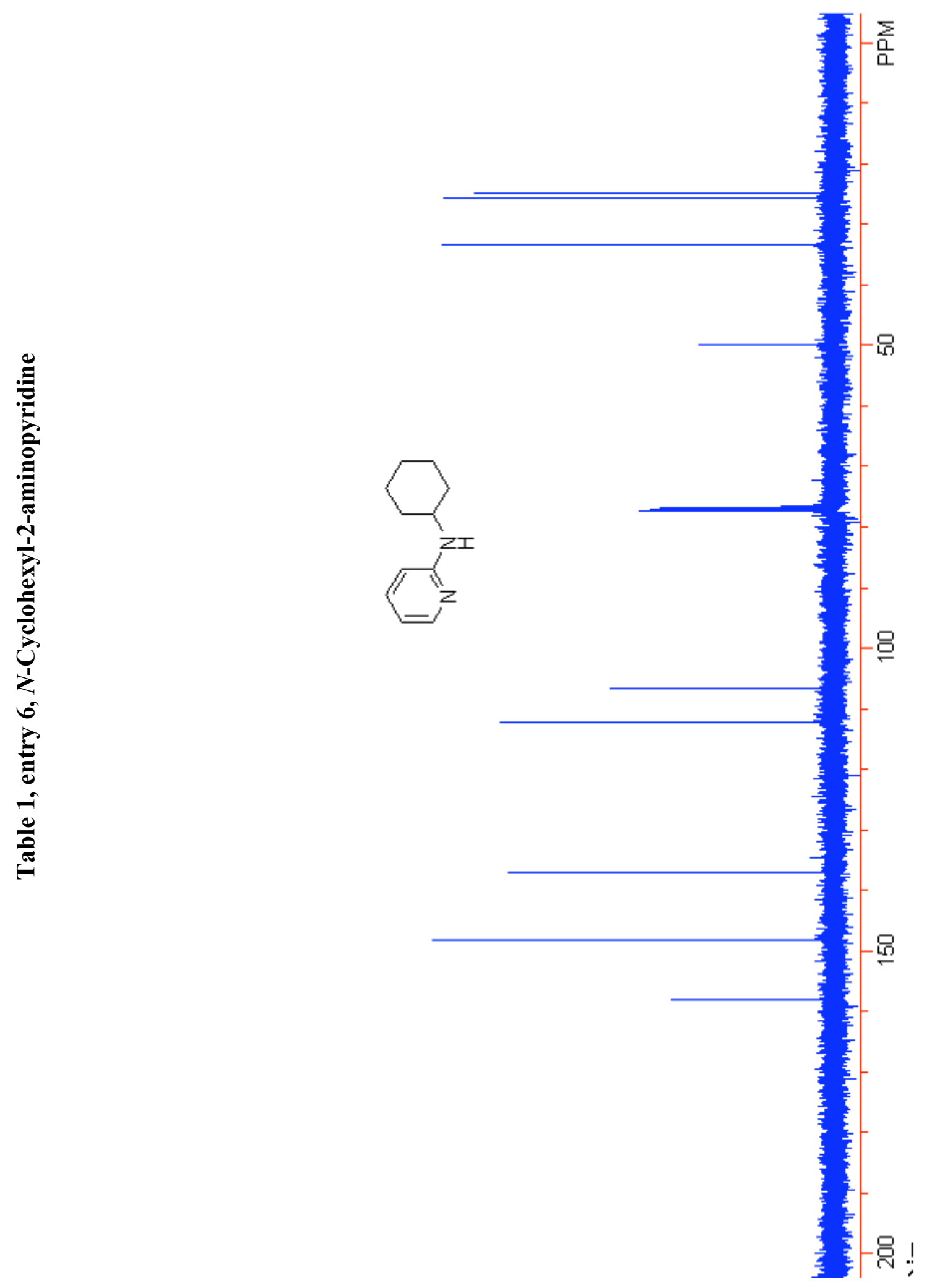


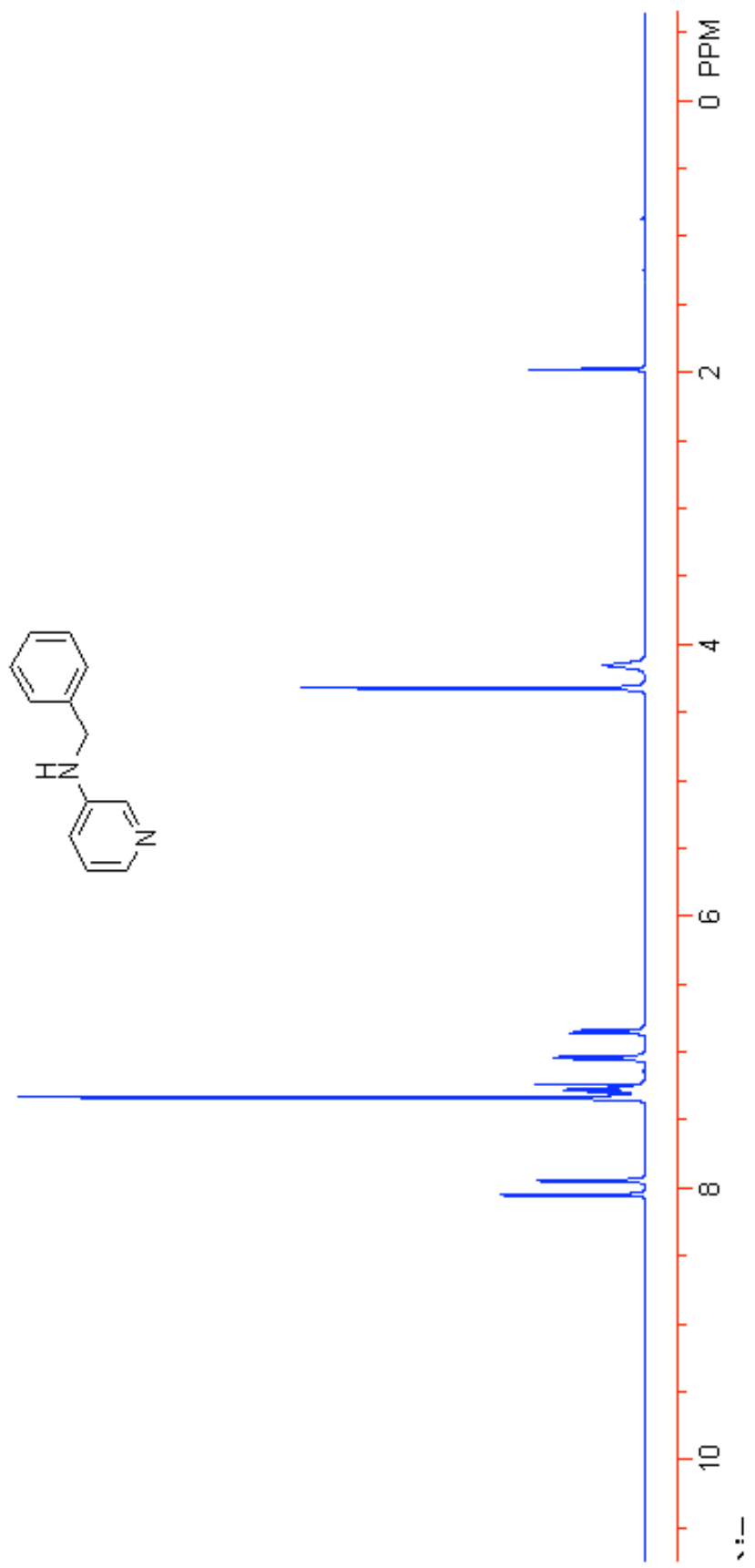



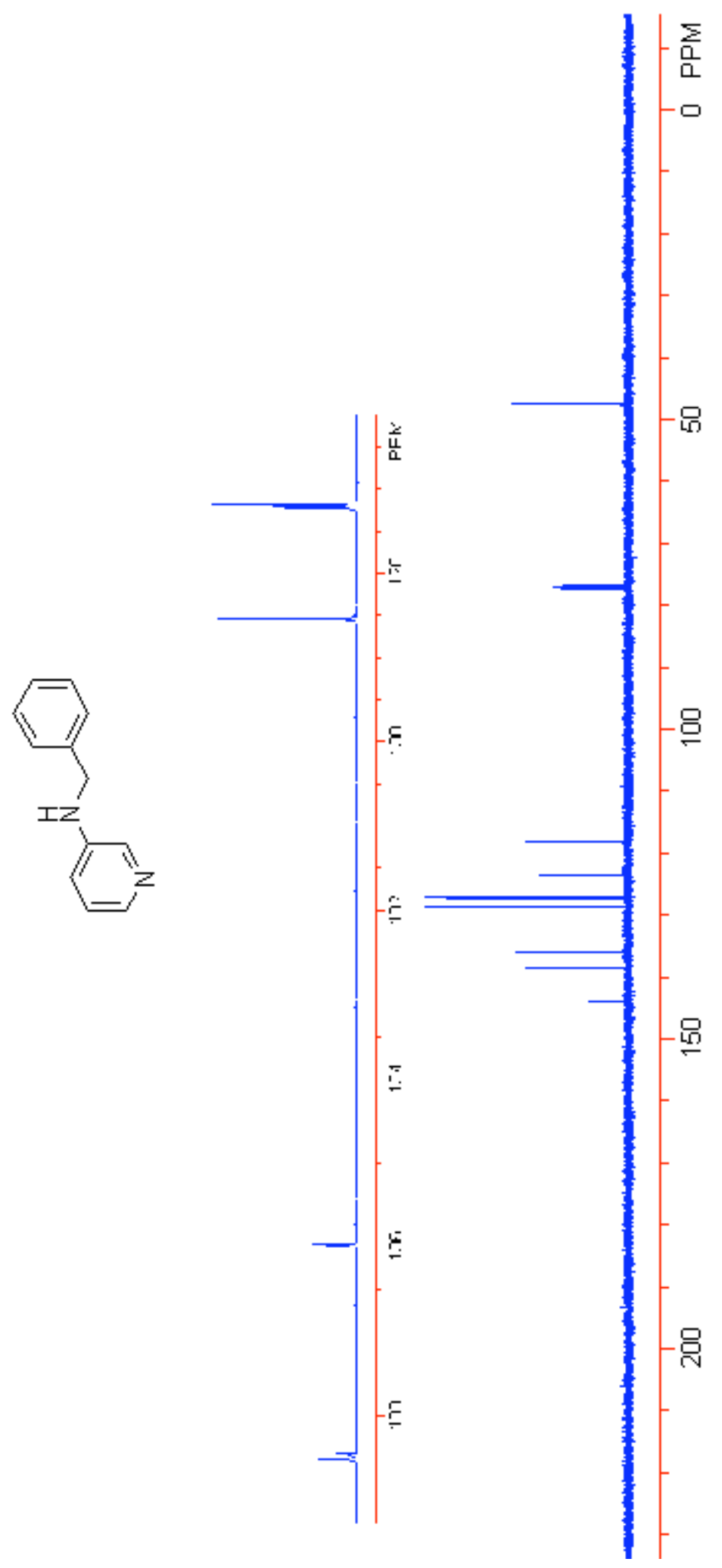


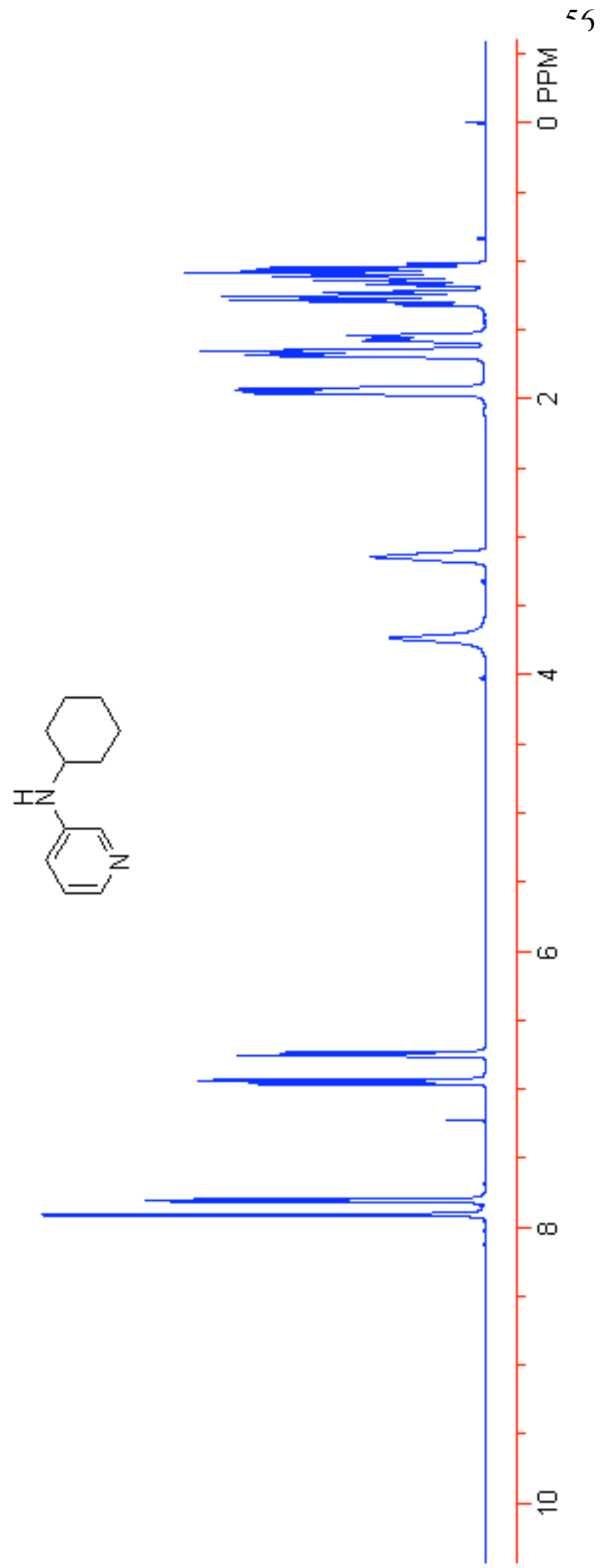



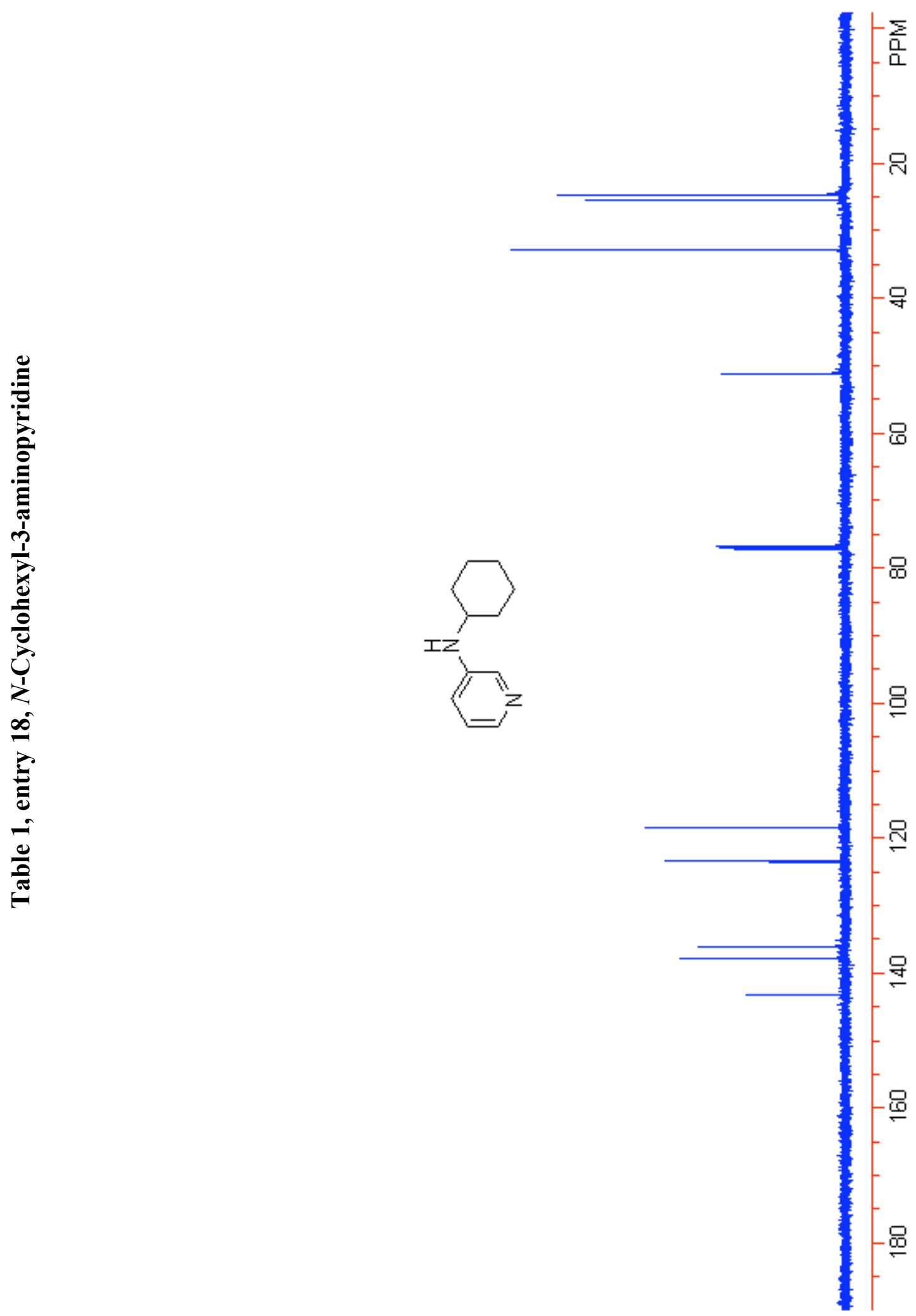


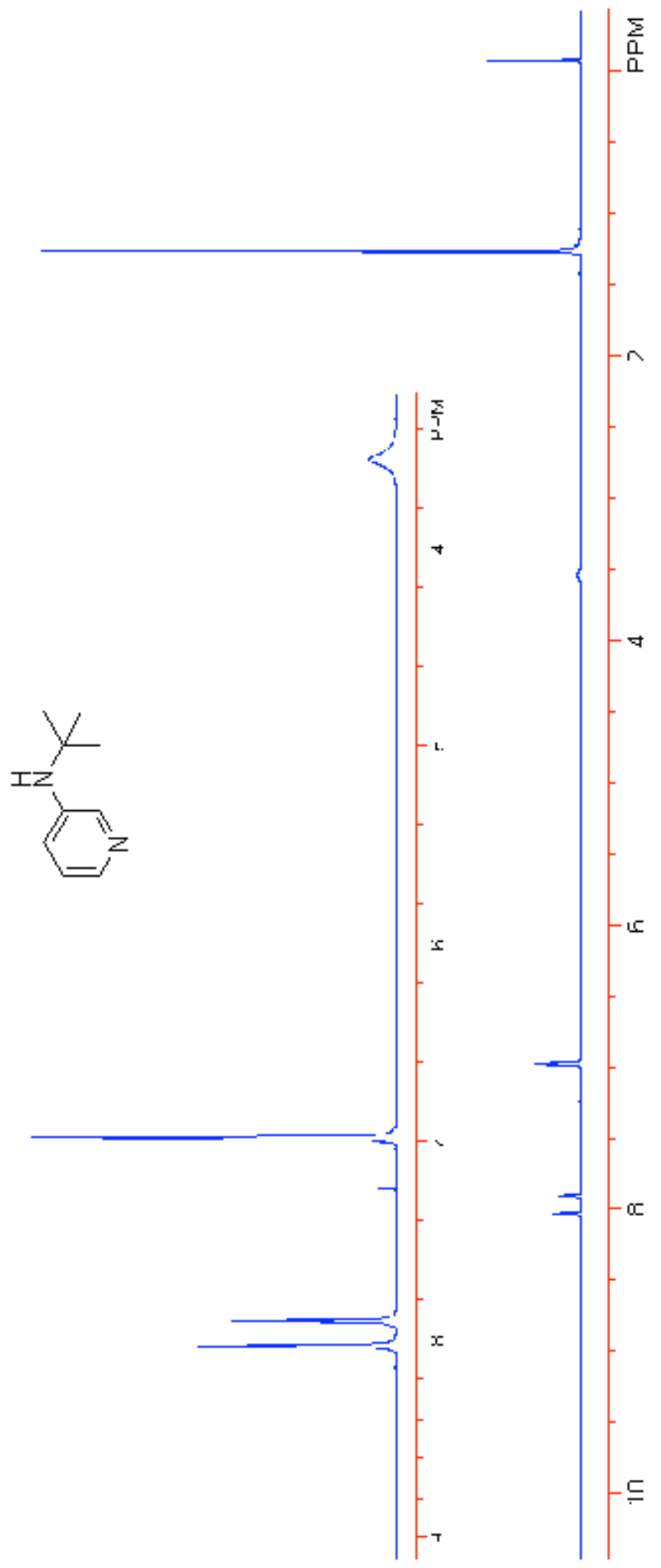



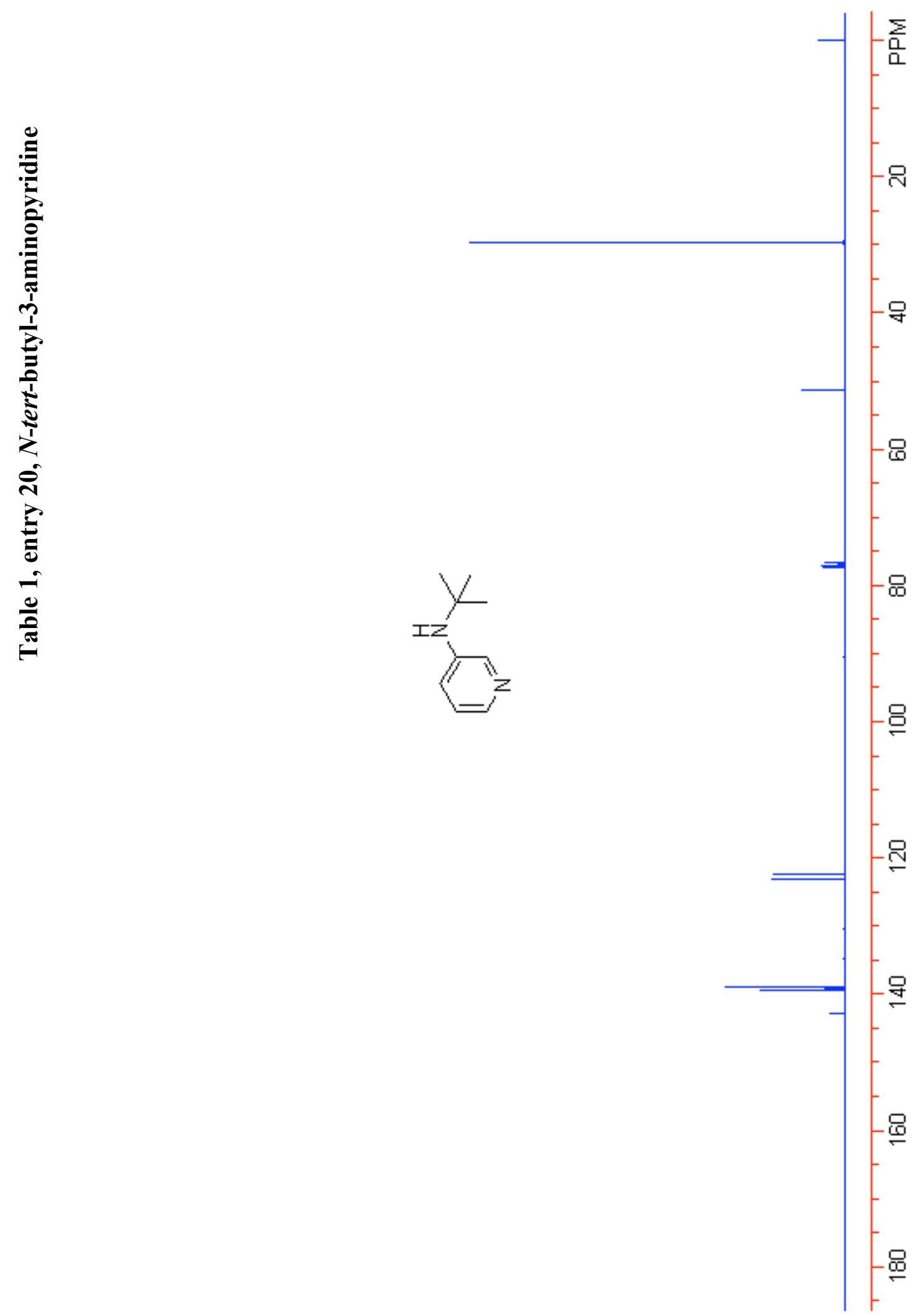


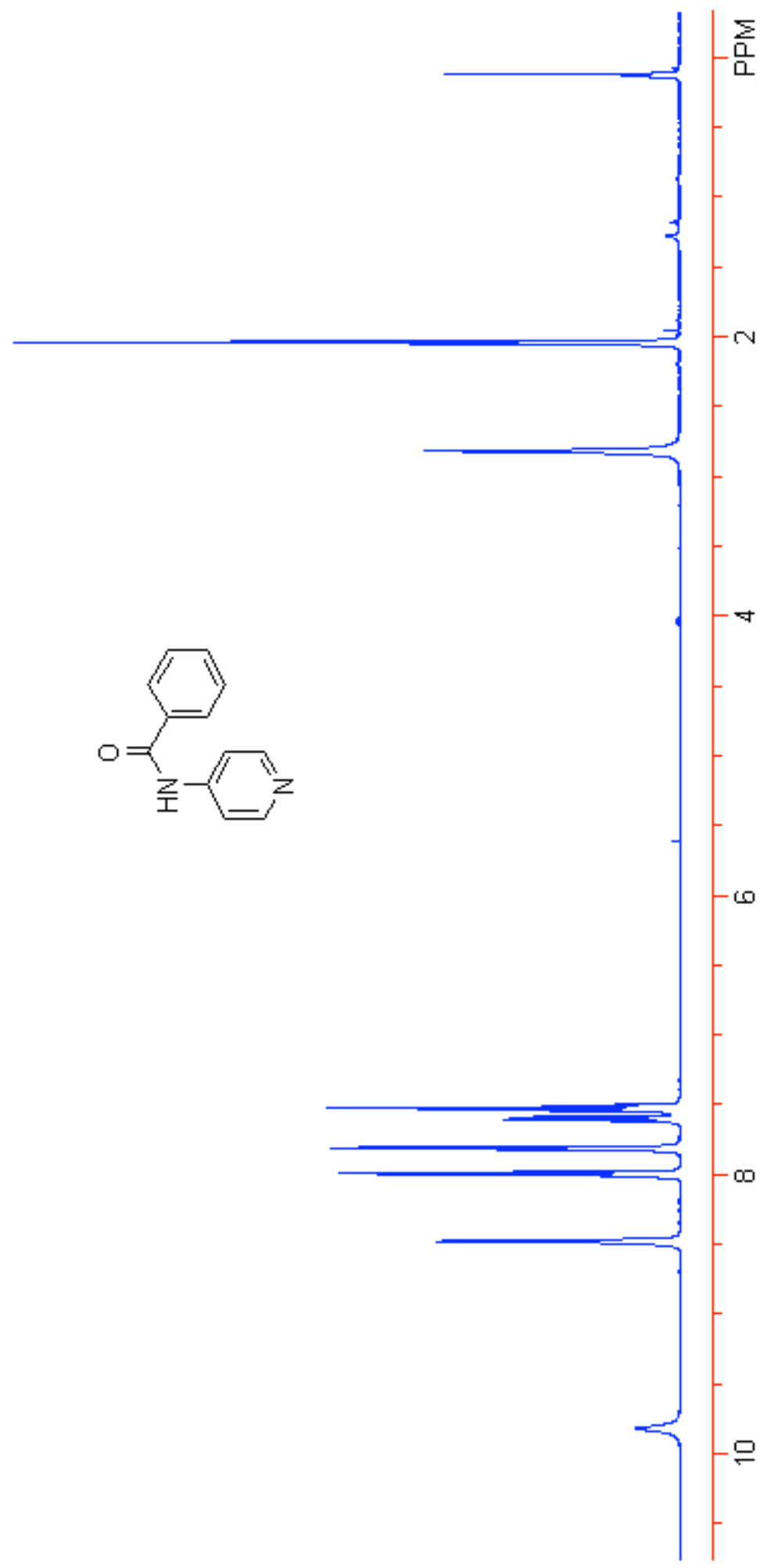




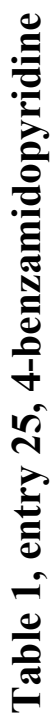

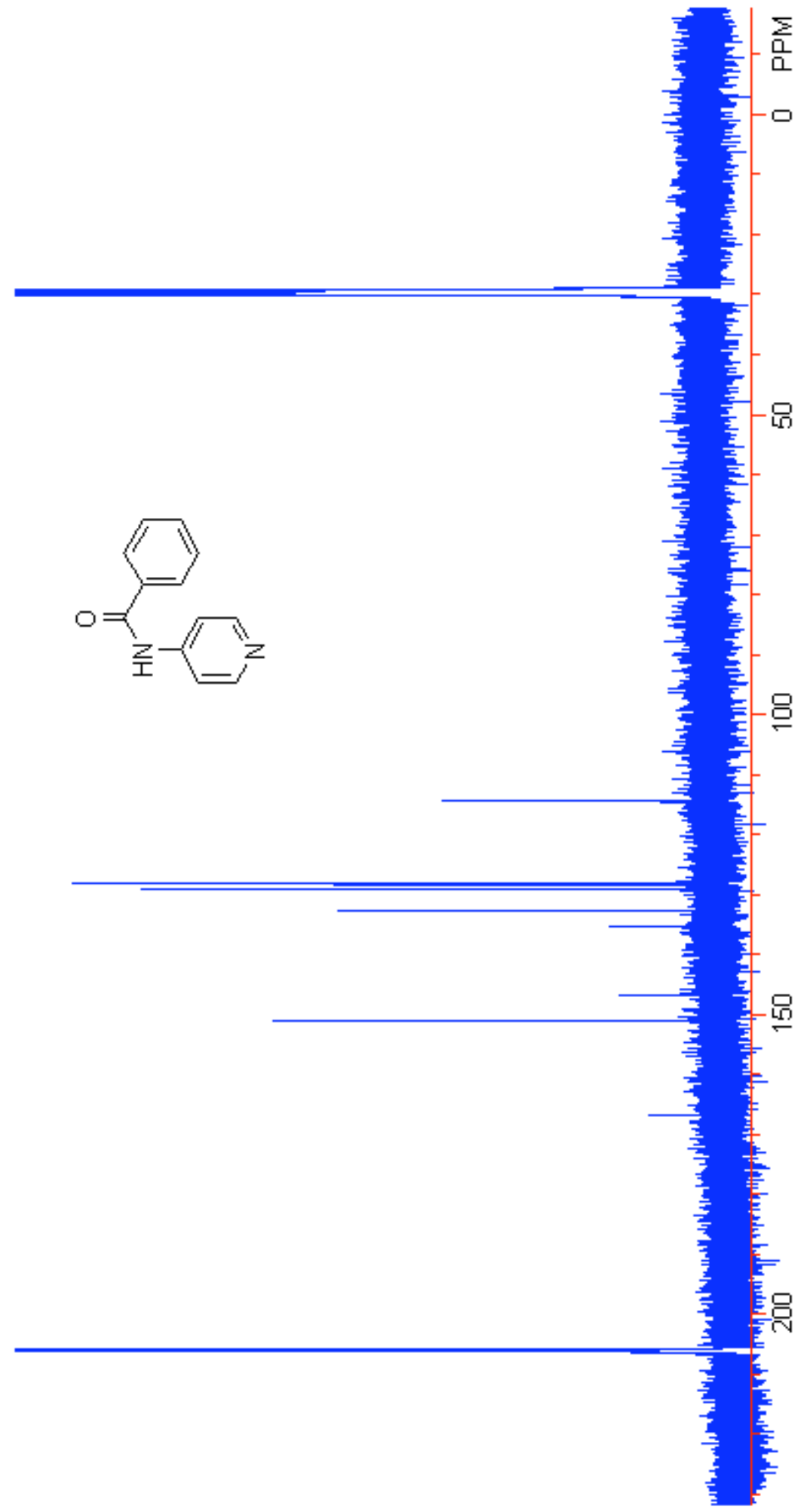




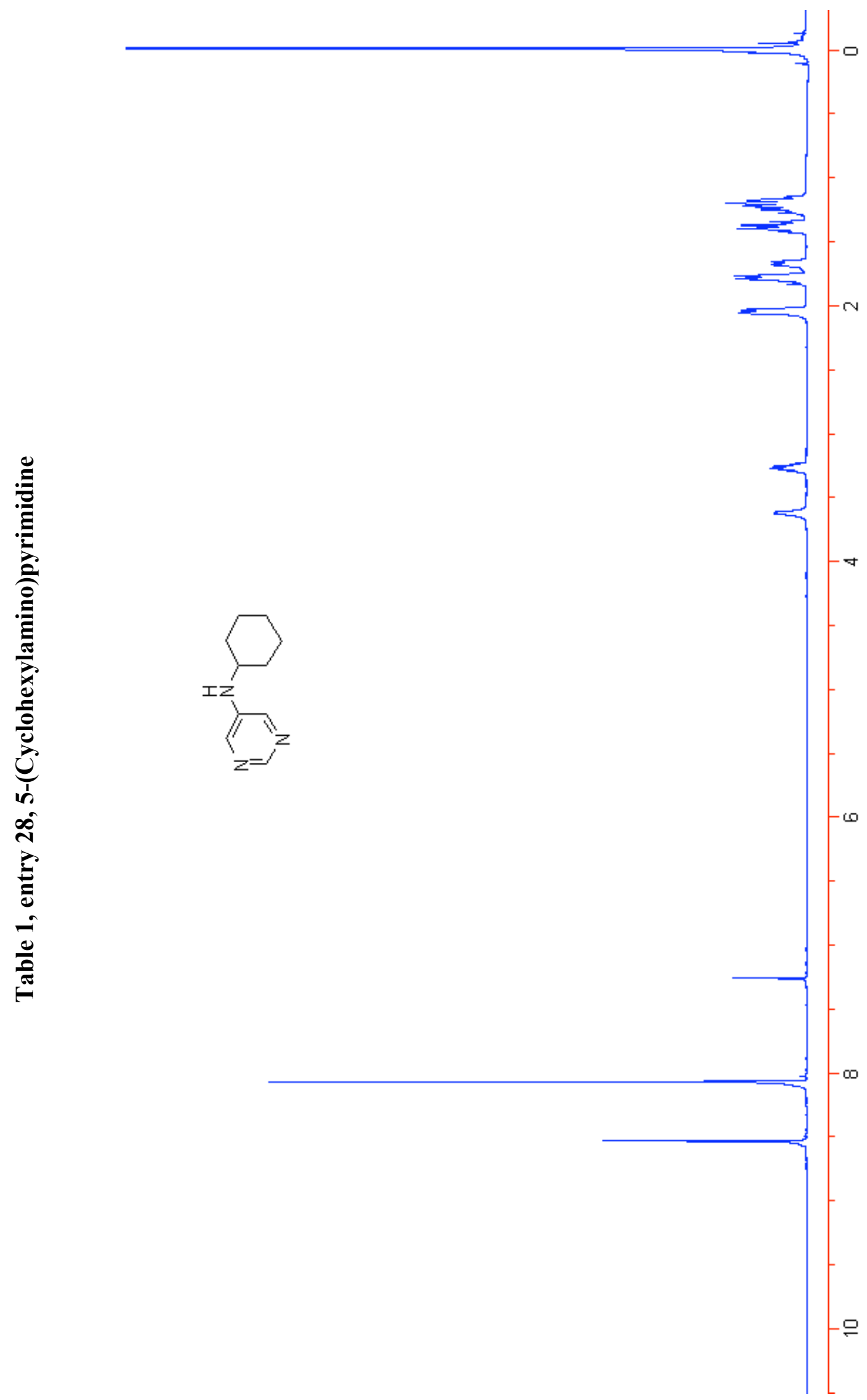




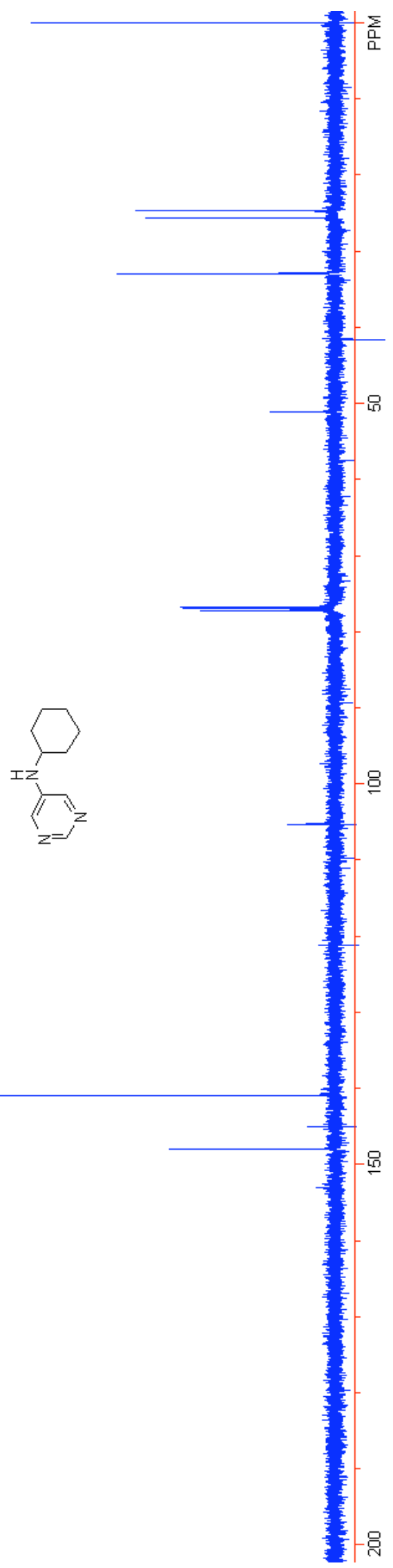




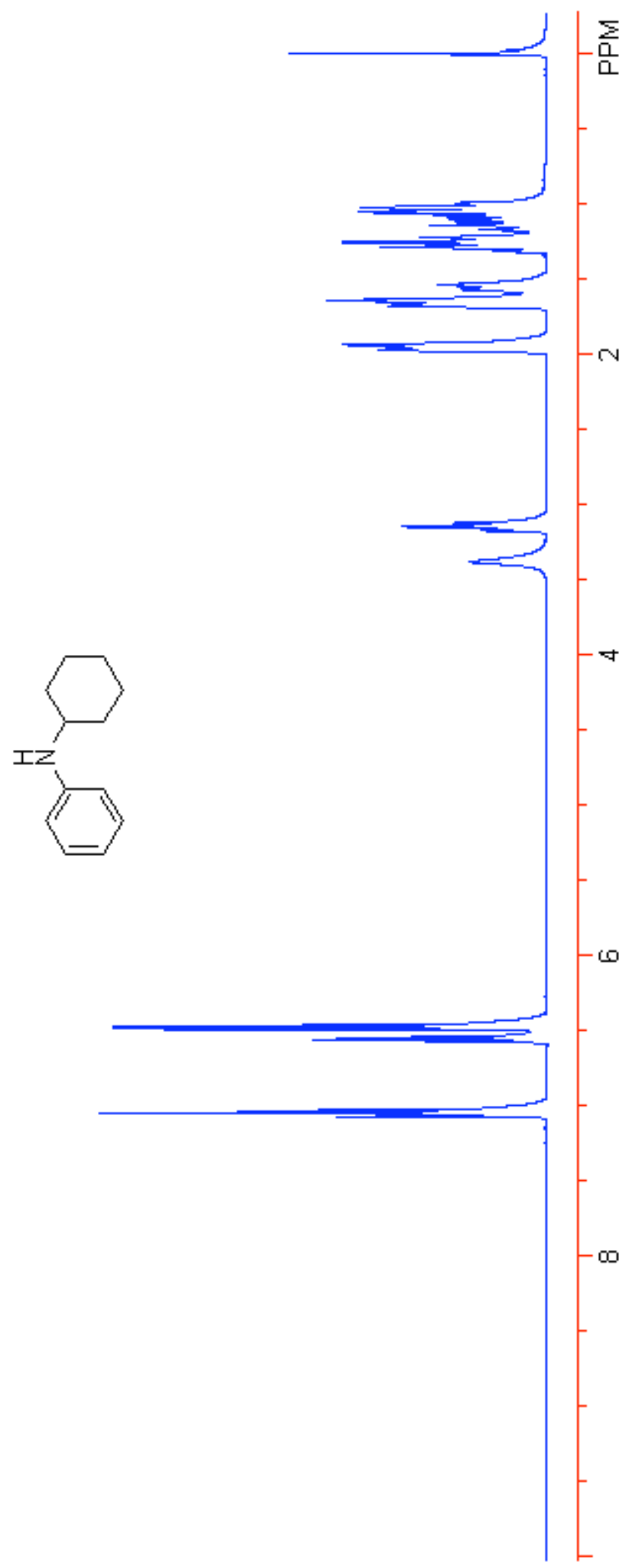




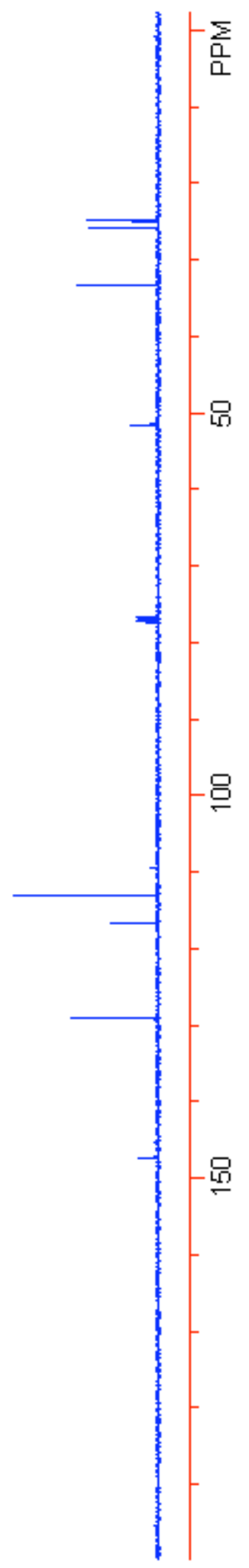




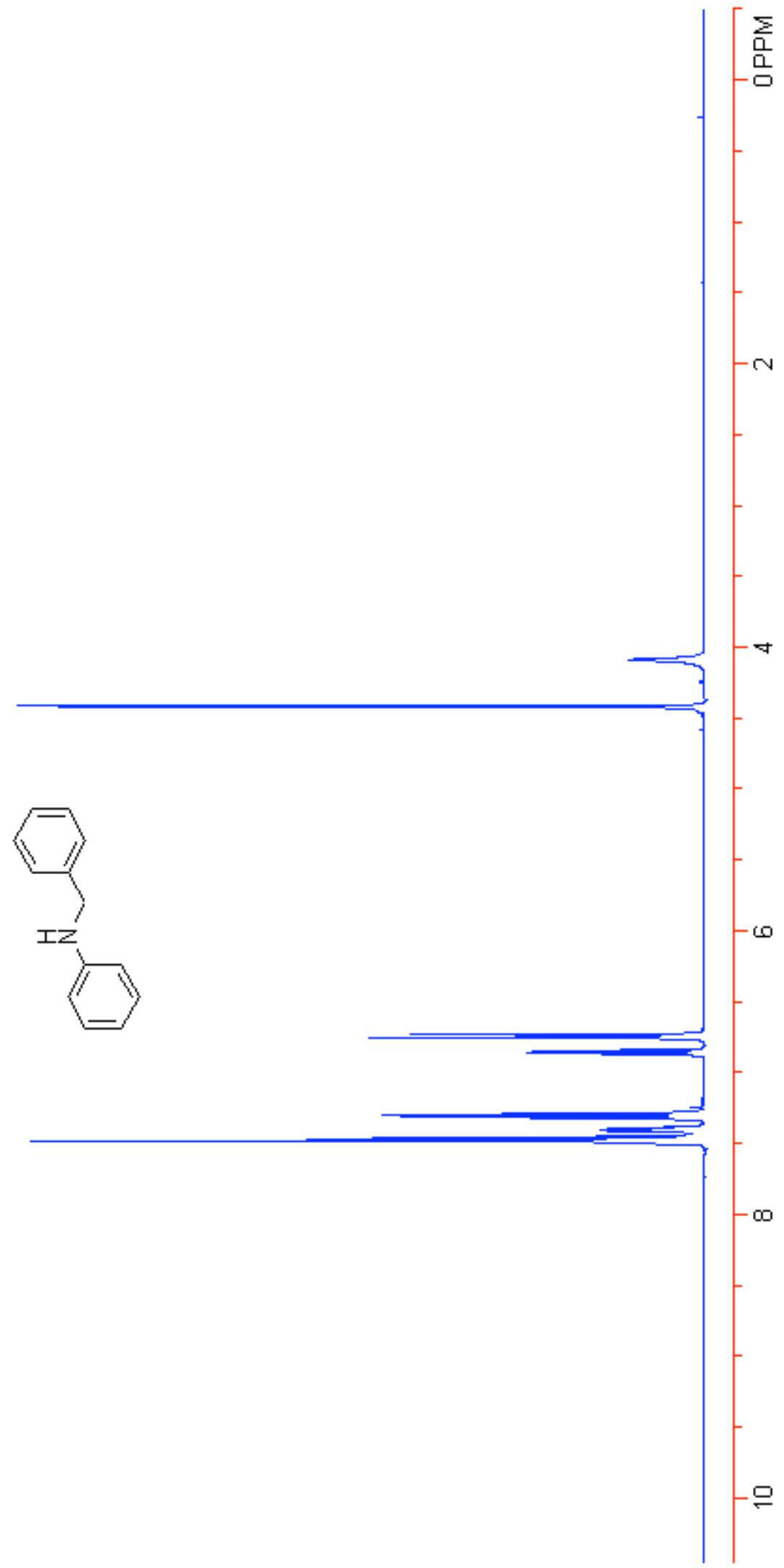




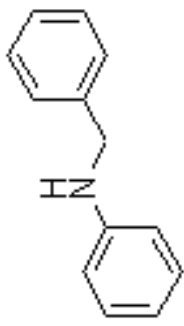

to

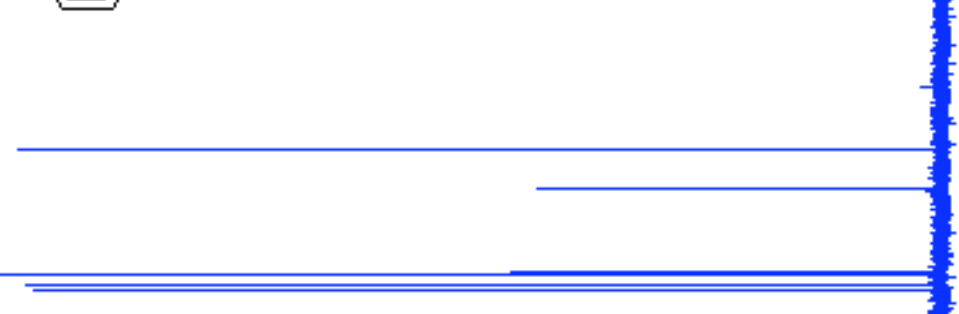

in

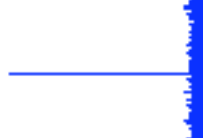




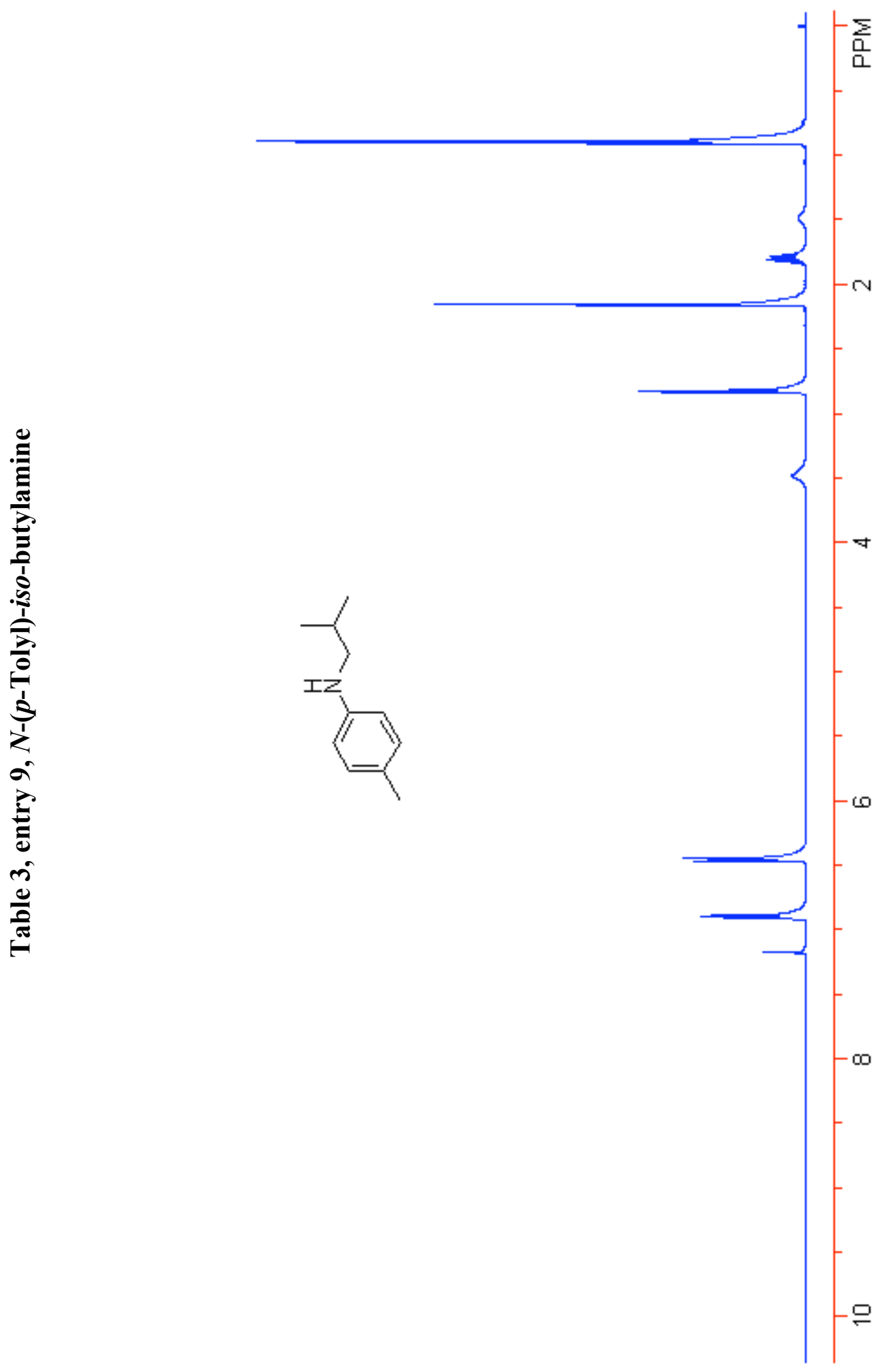



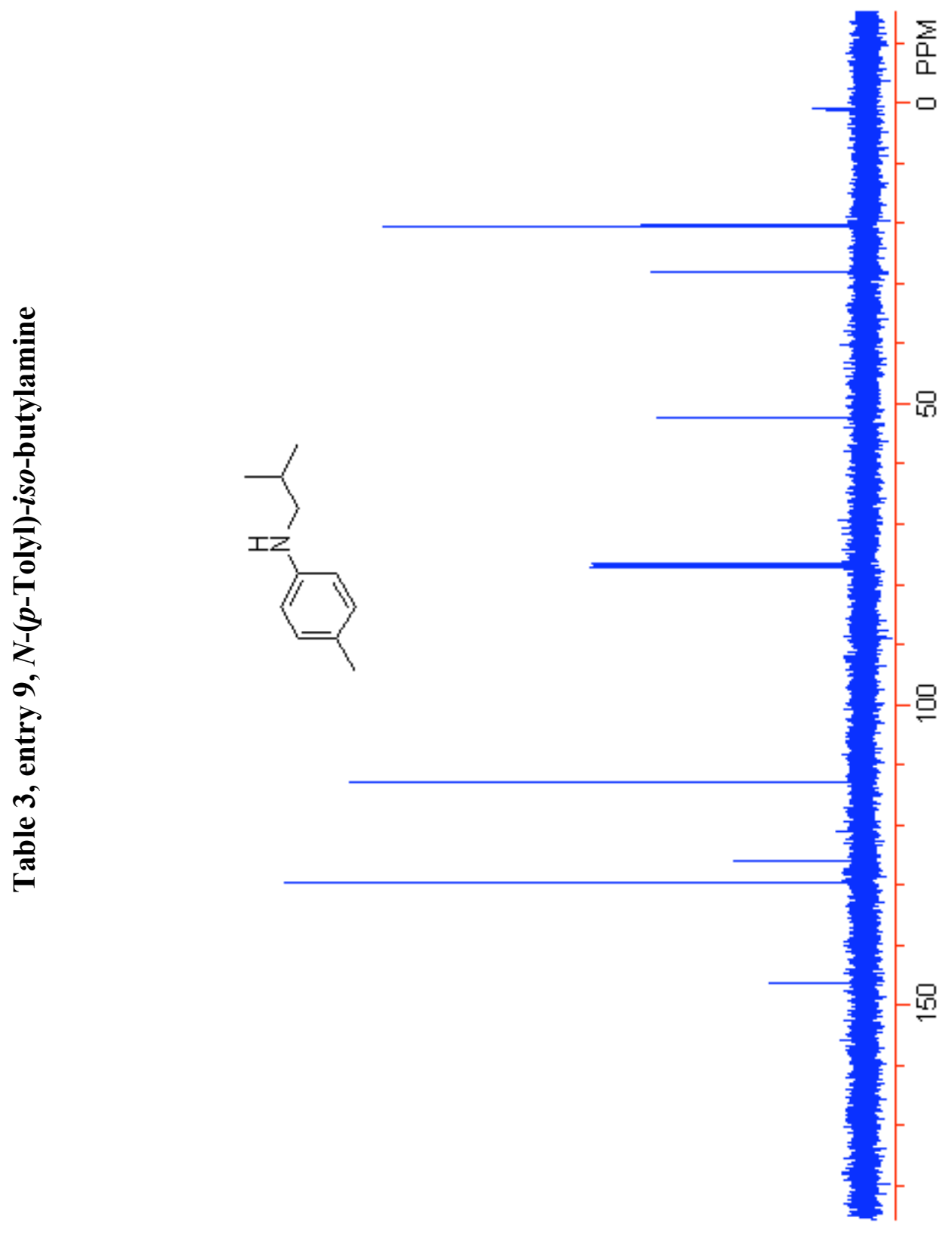


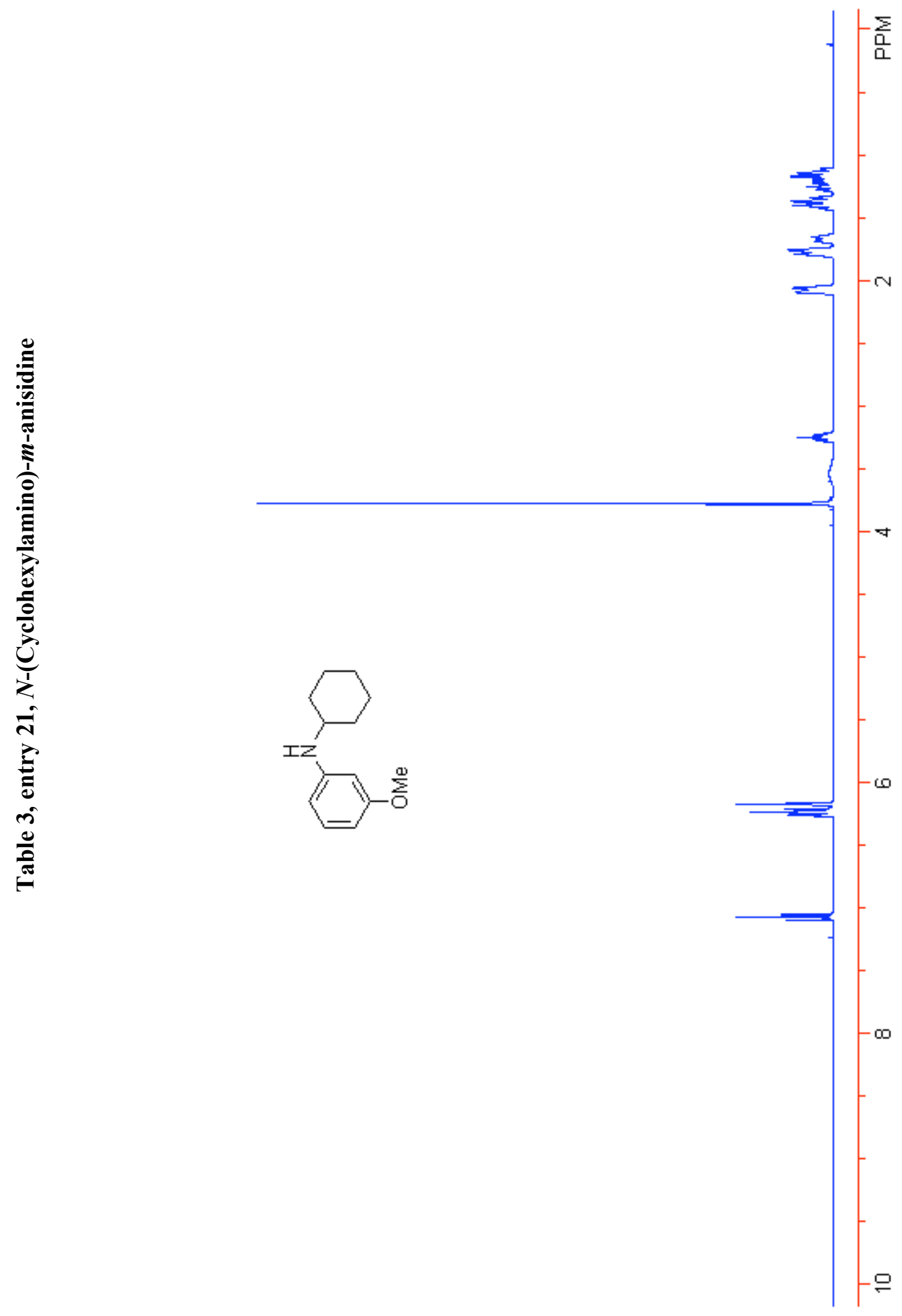




$$
1.1
$$




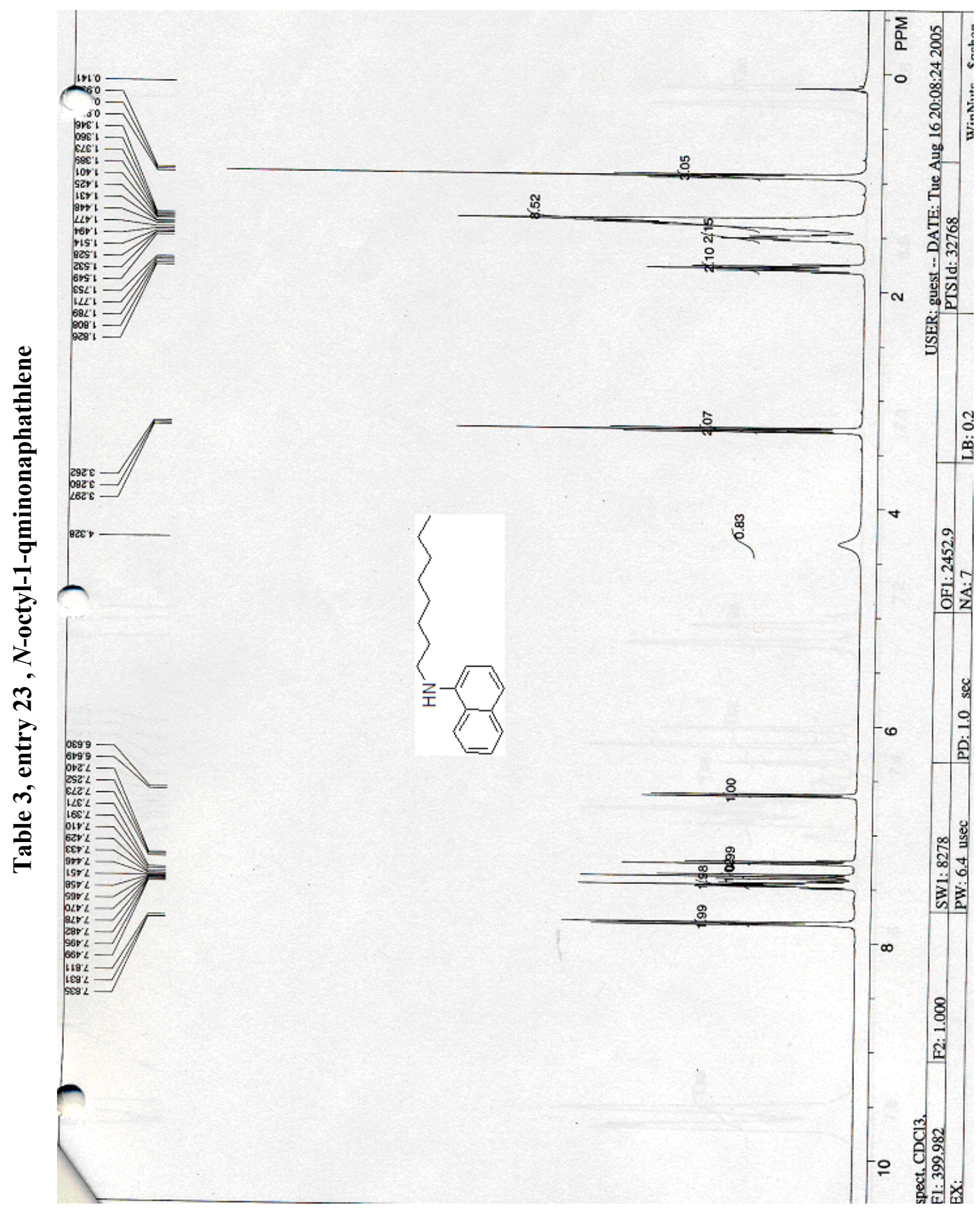




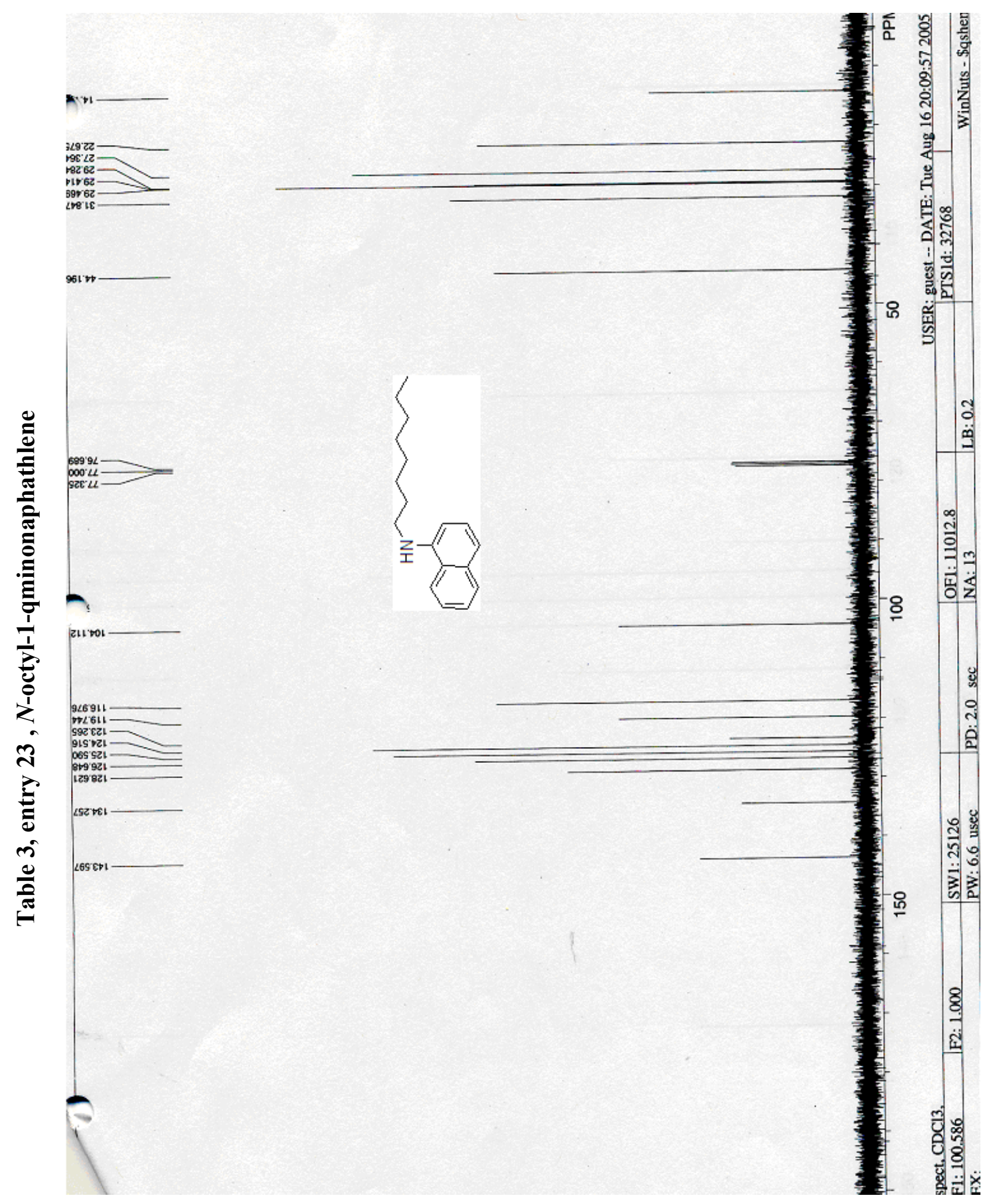




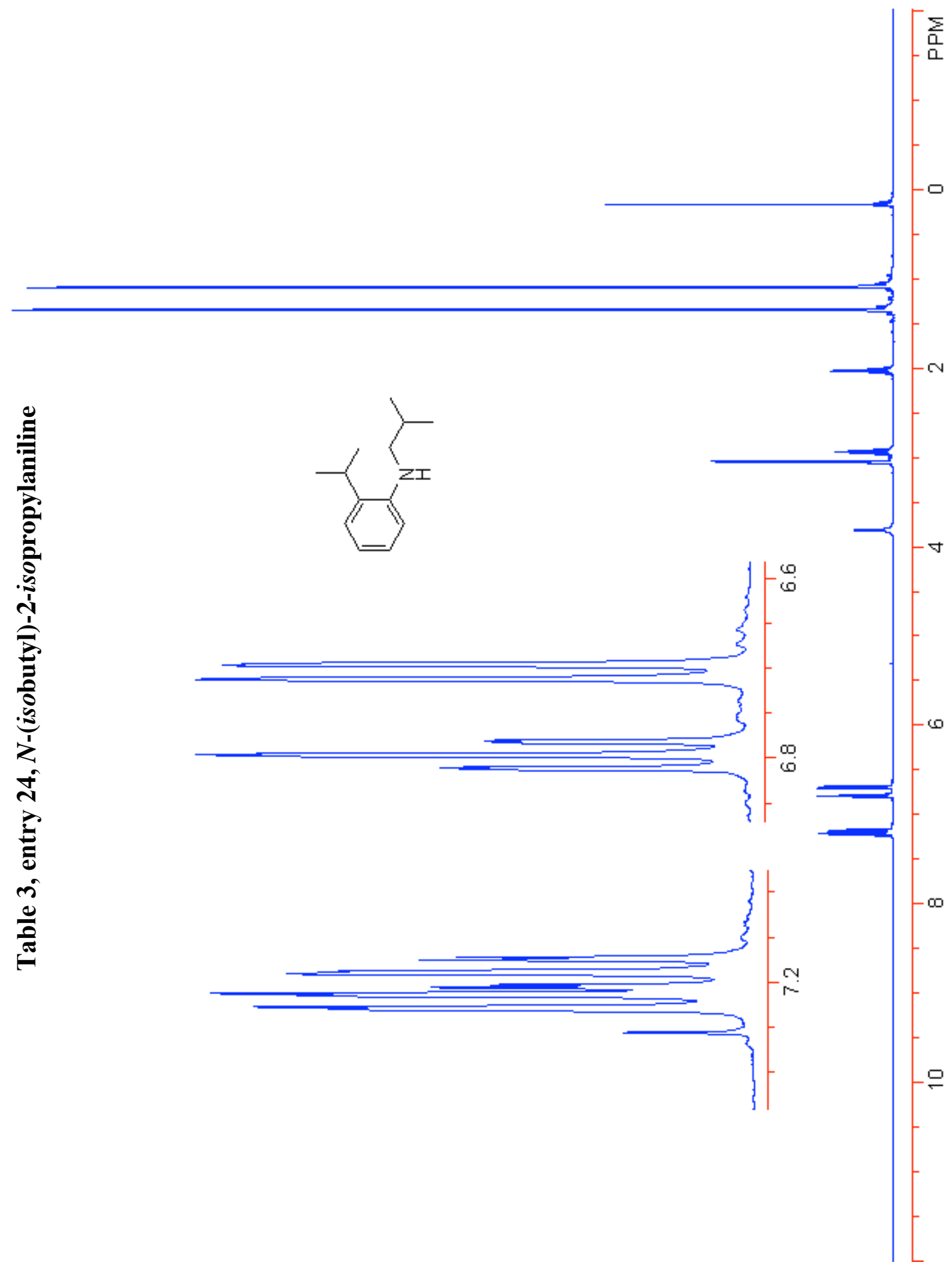




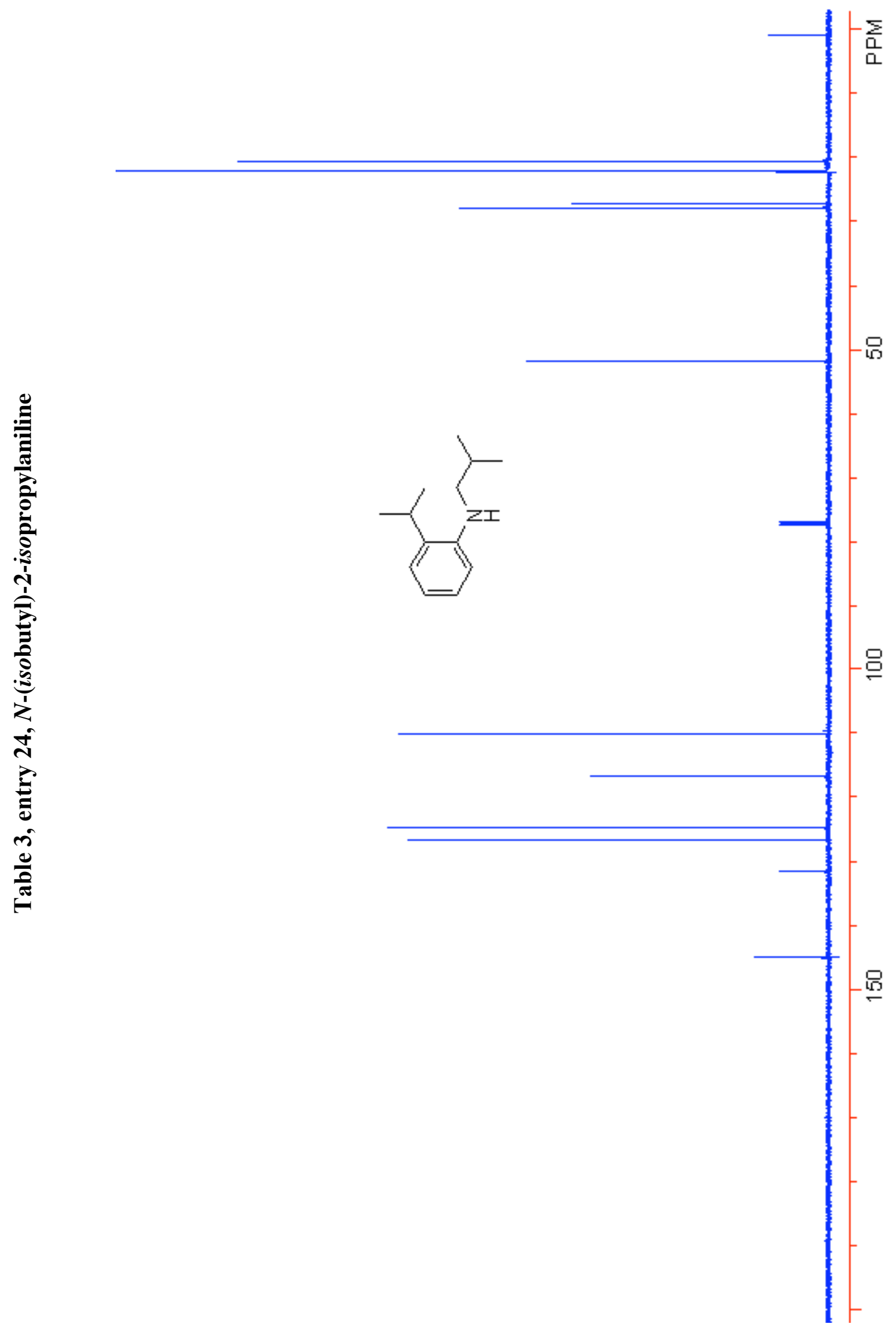




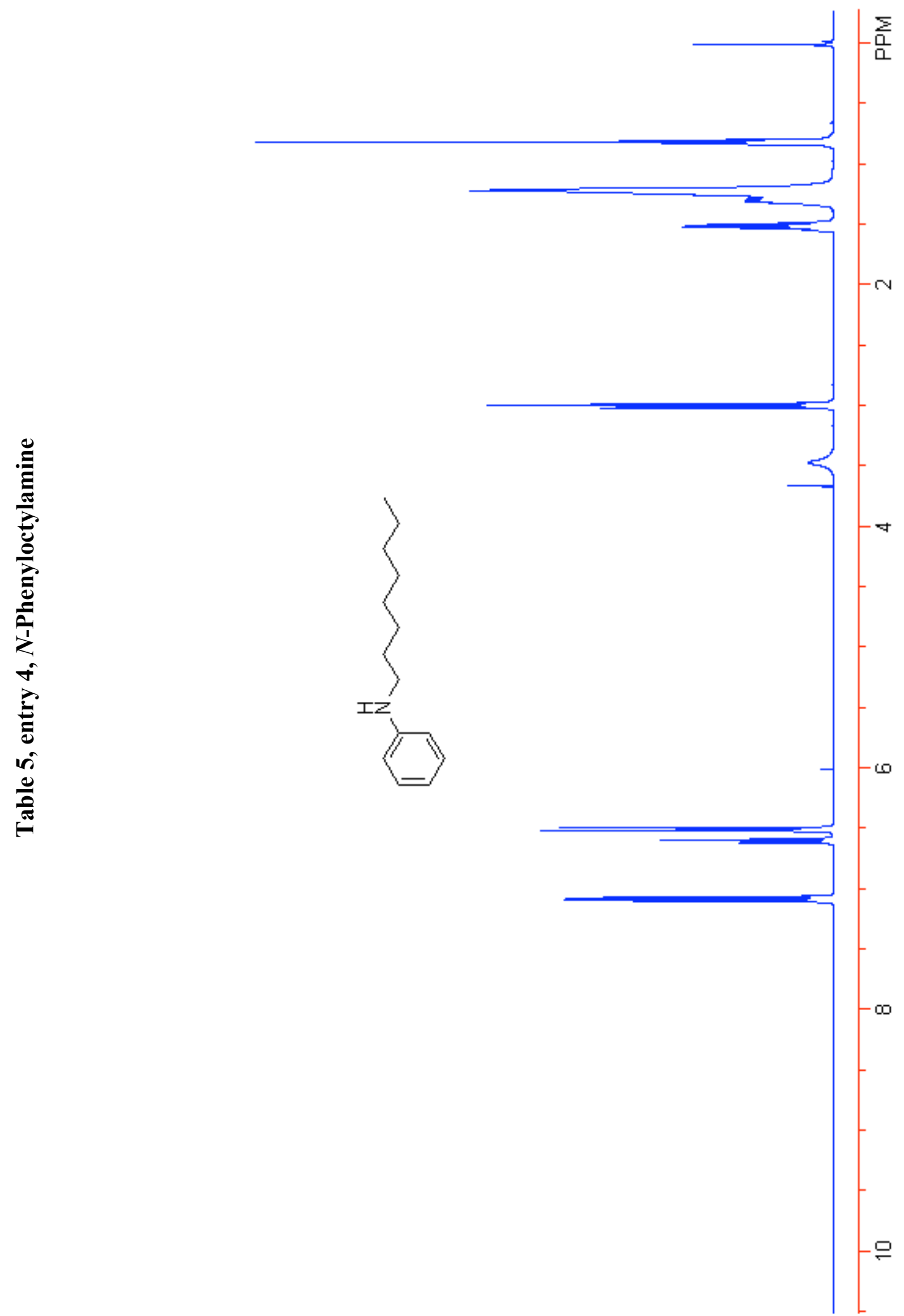



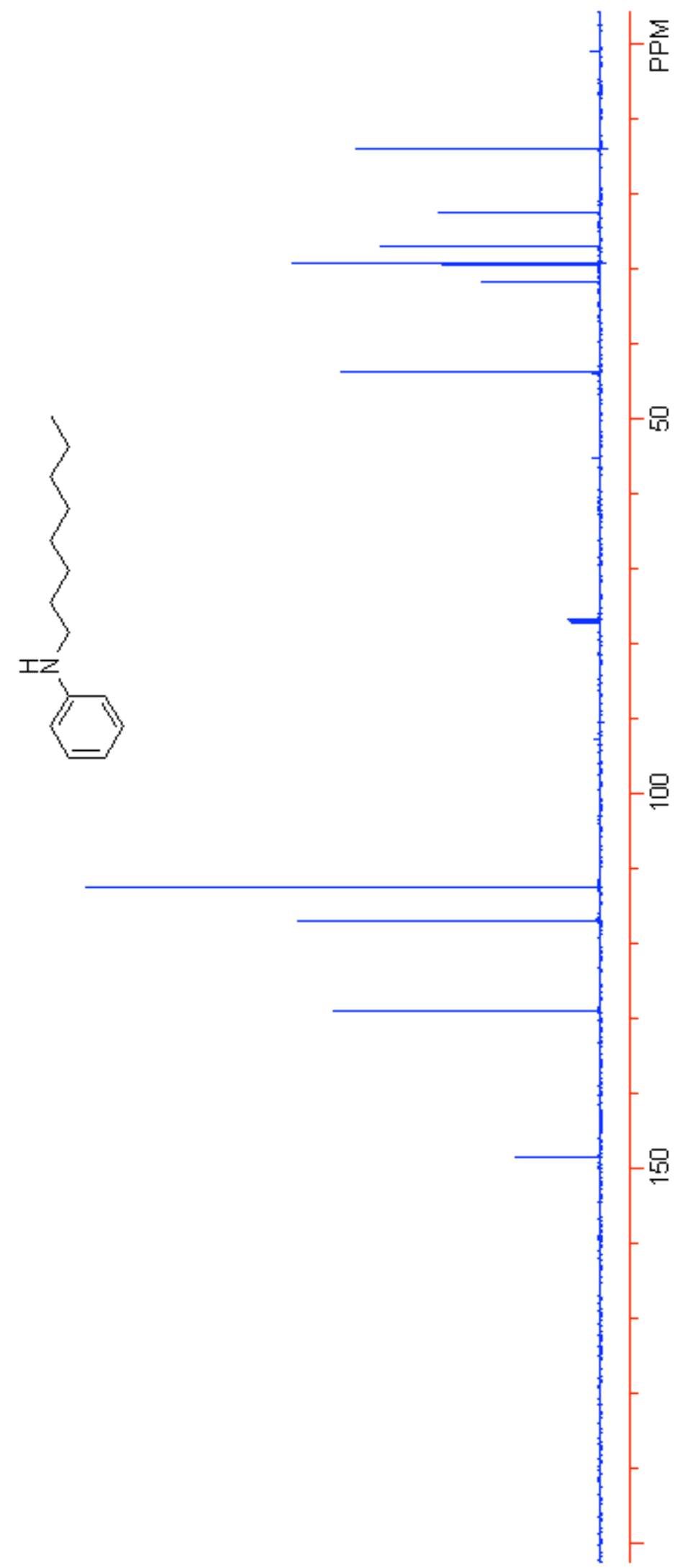


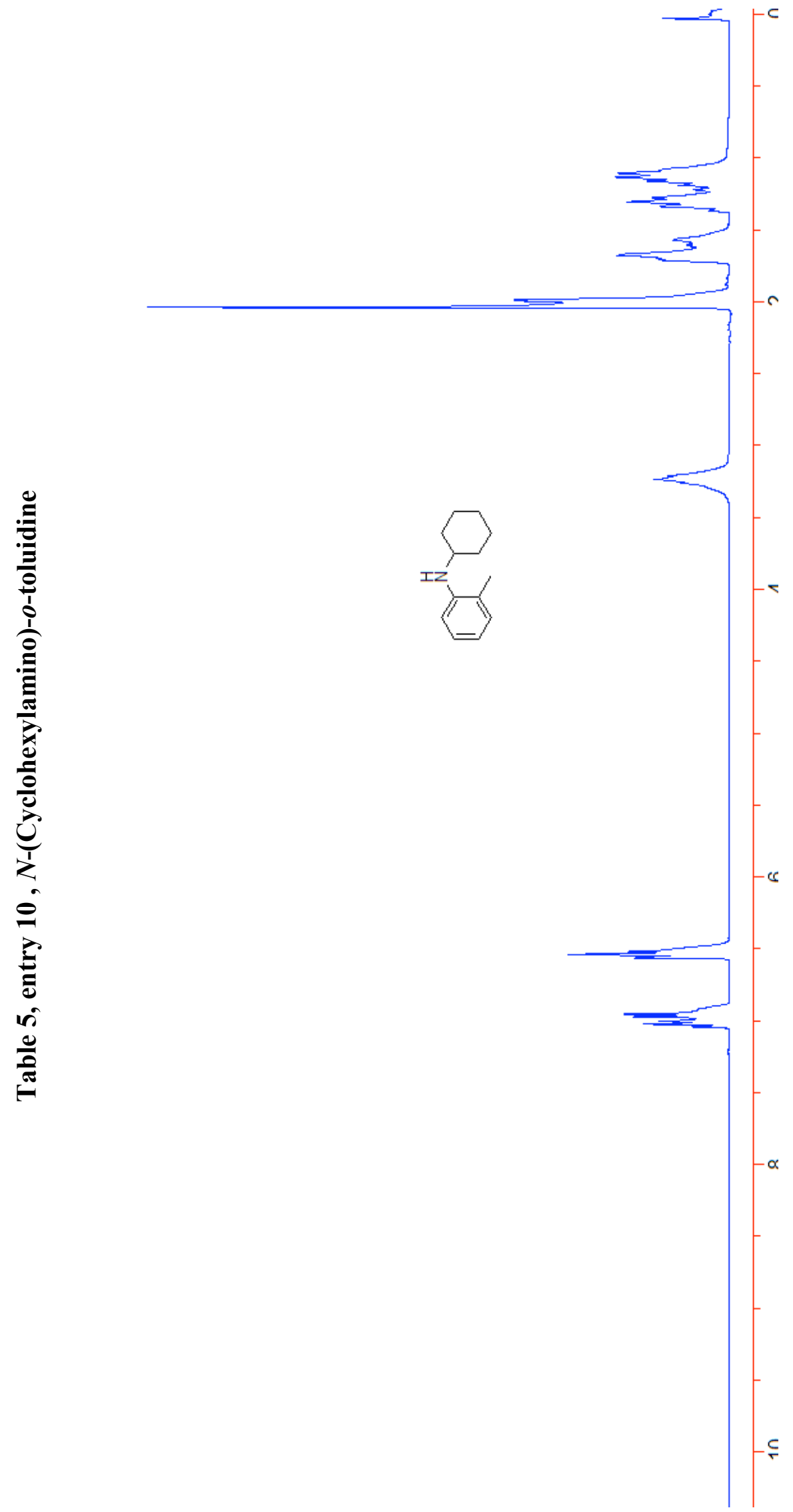




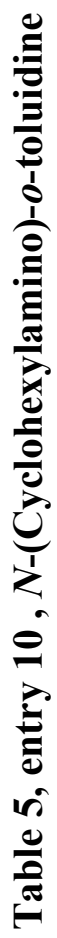

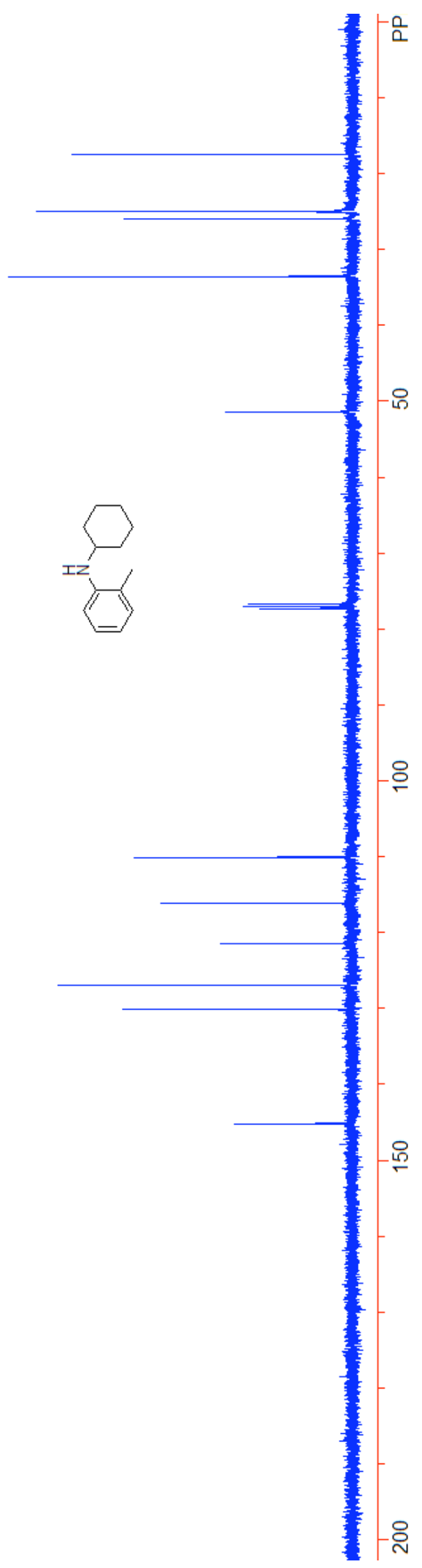




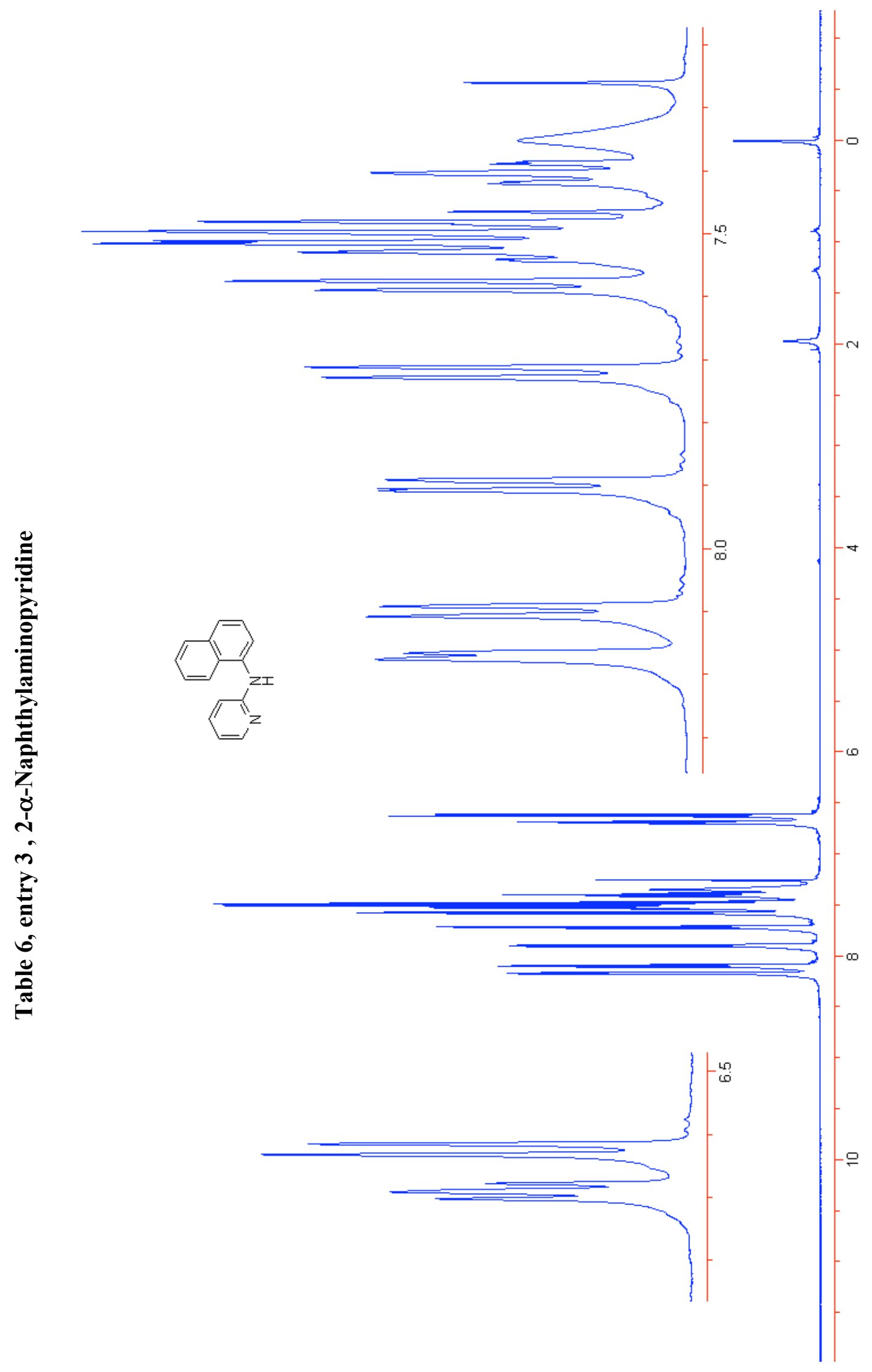




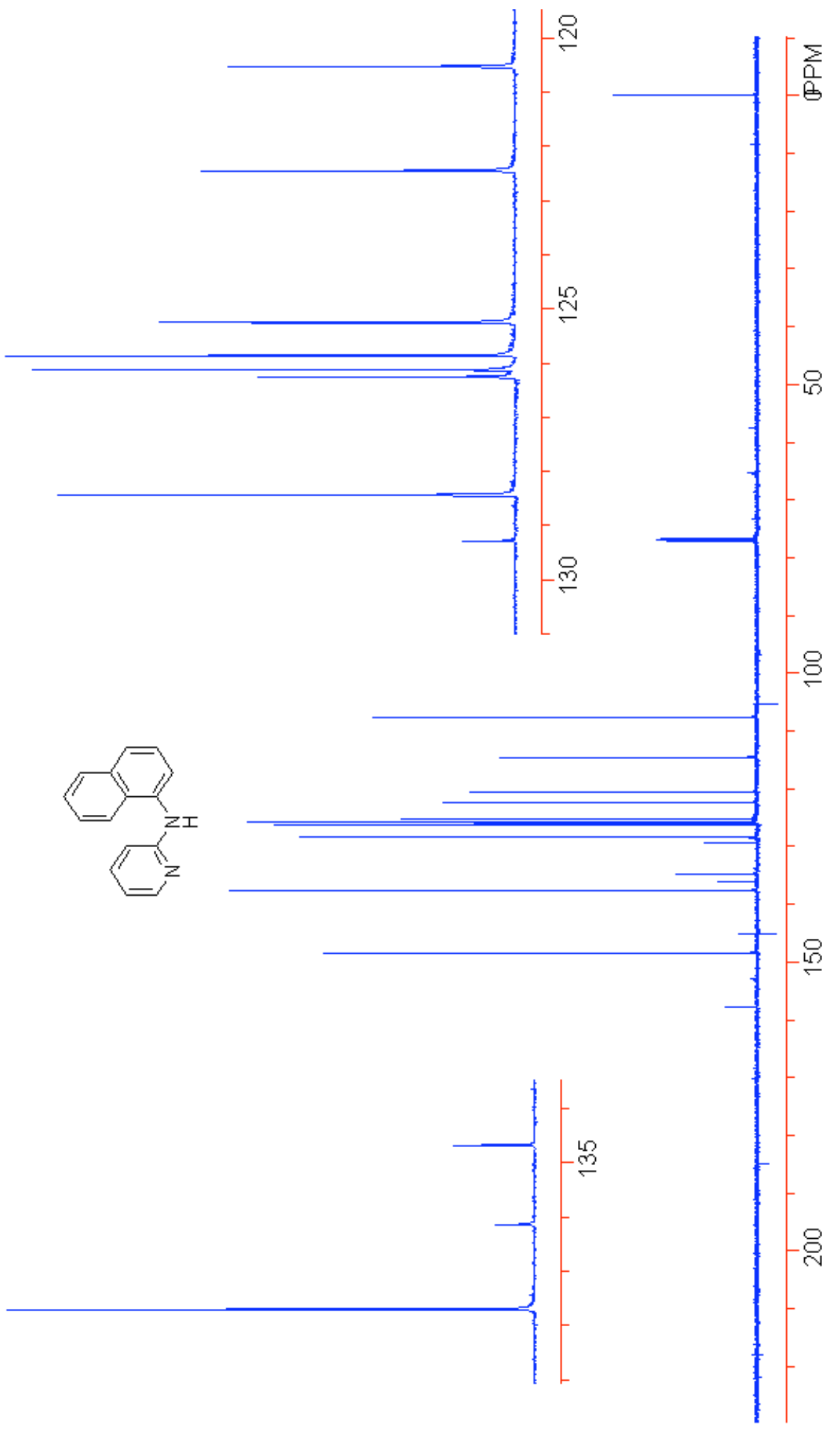




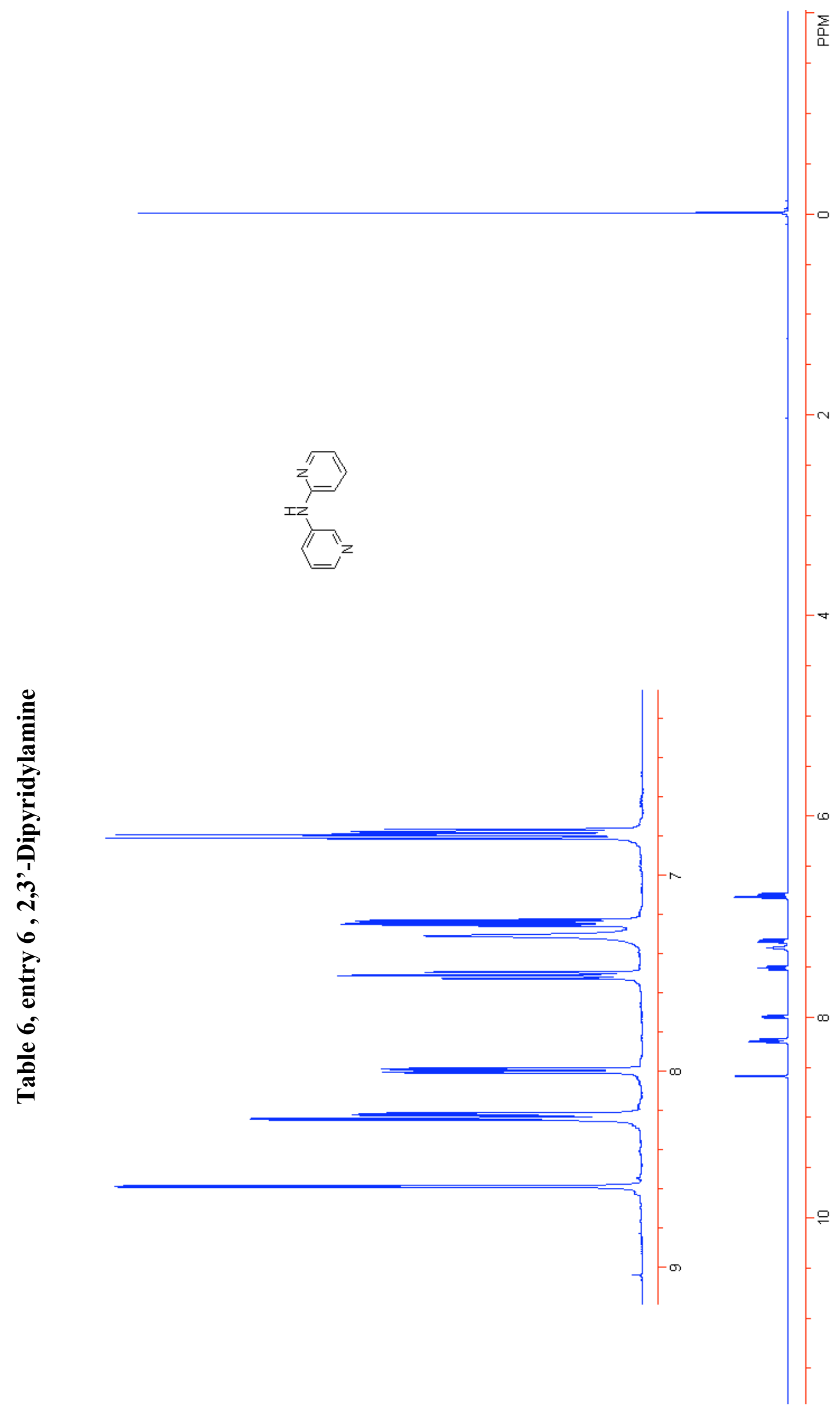




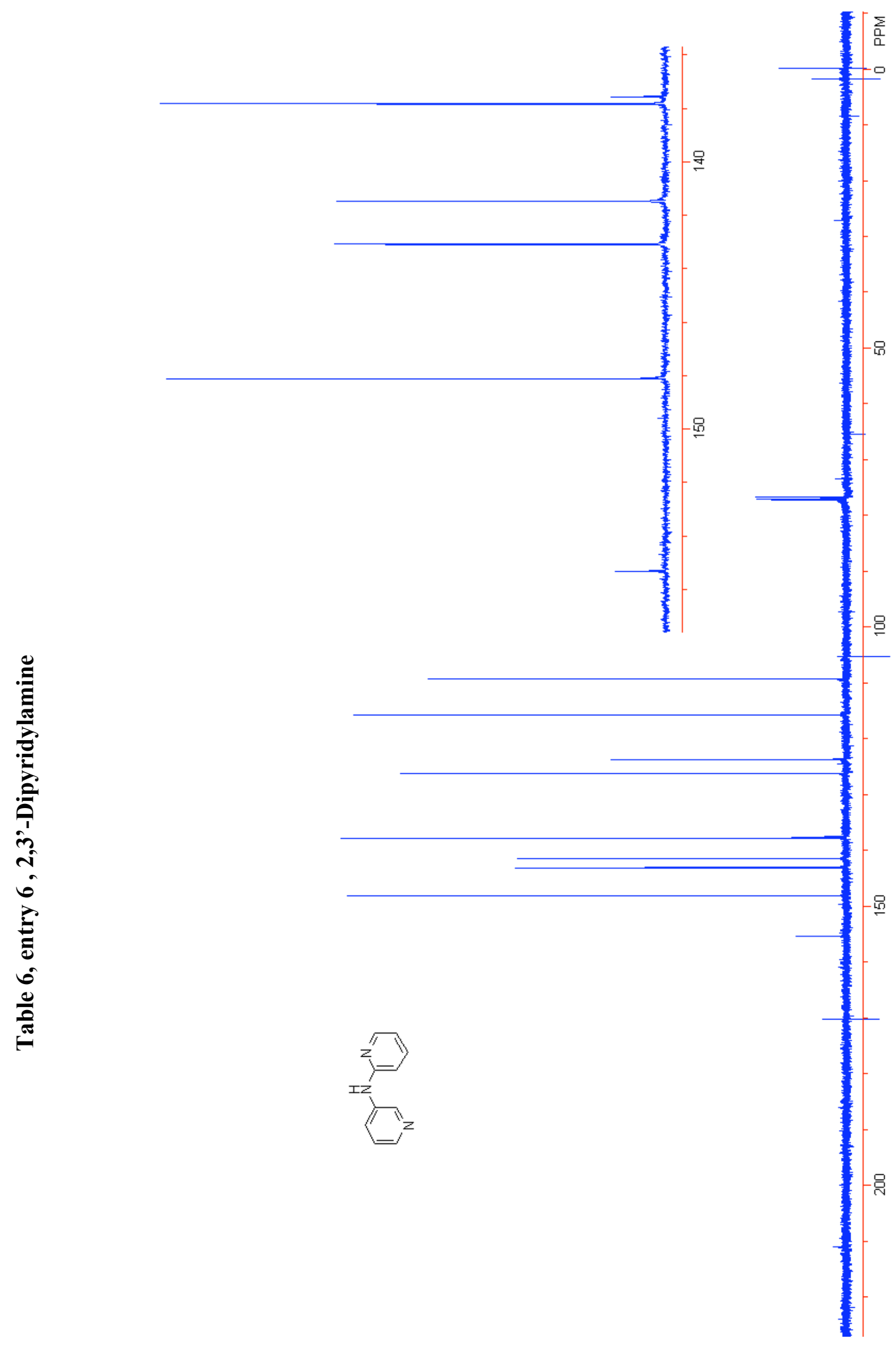




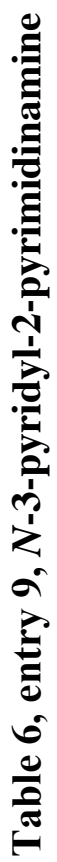

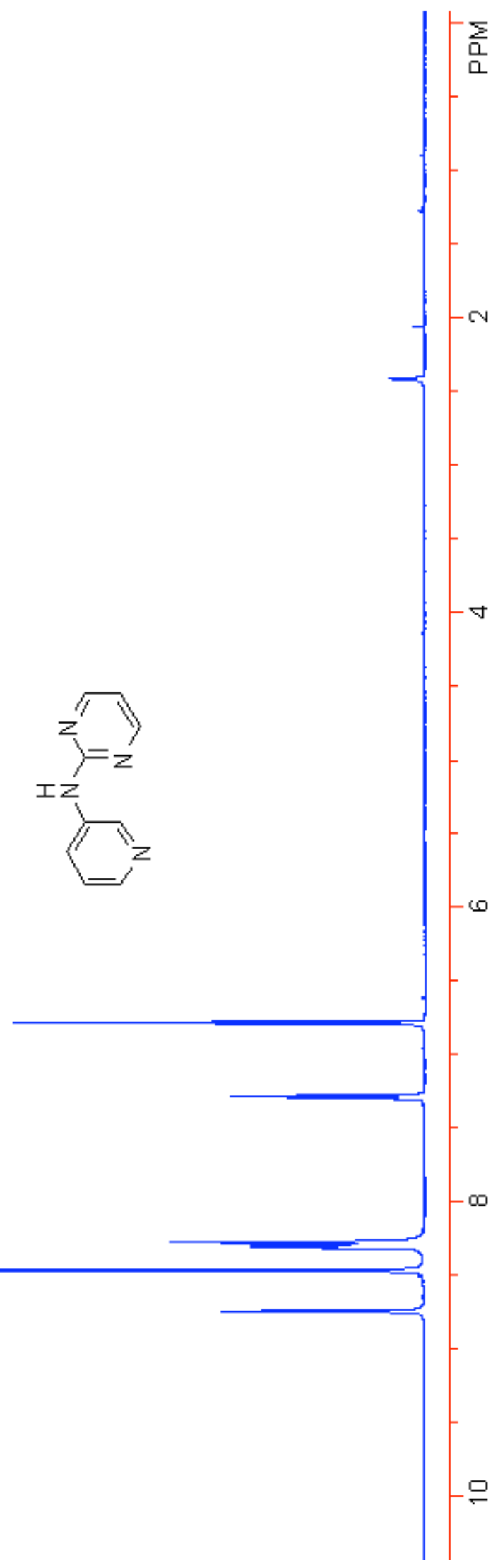




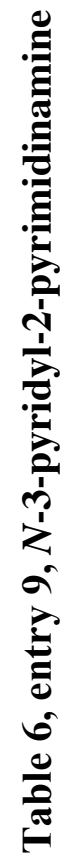

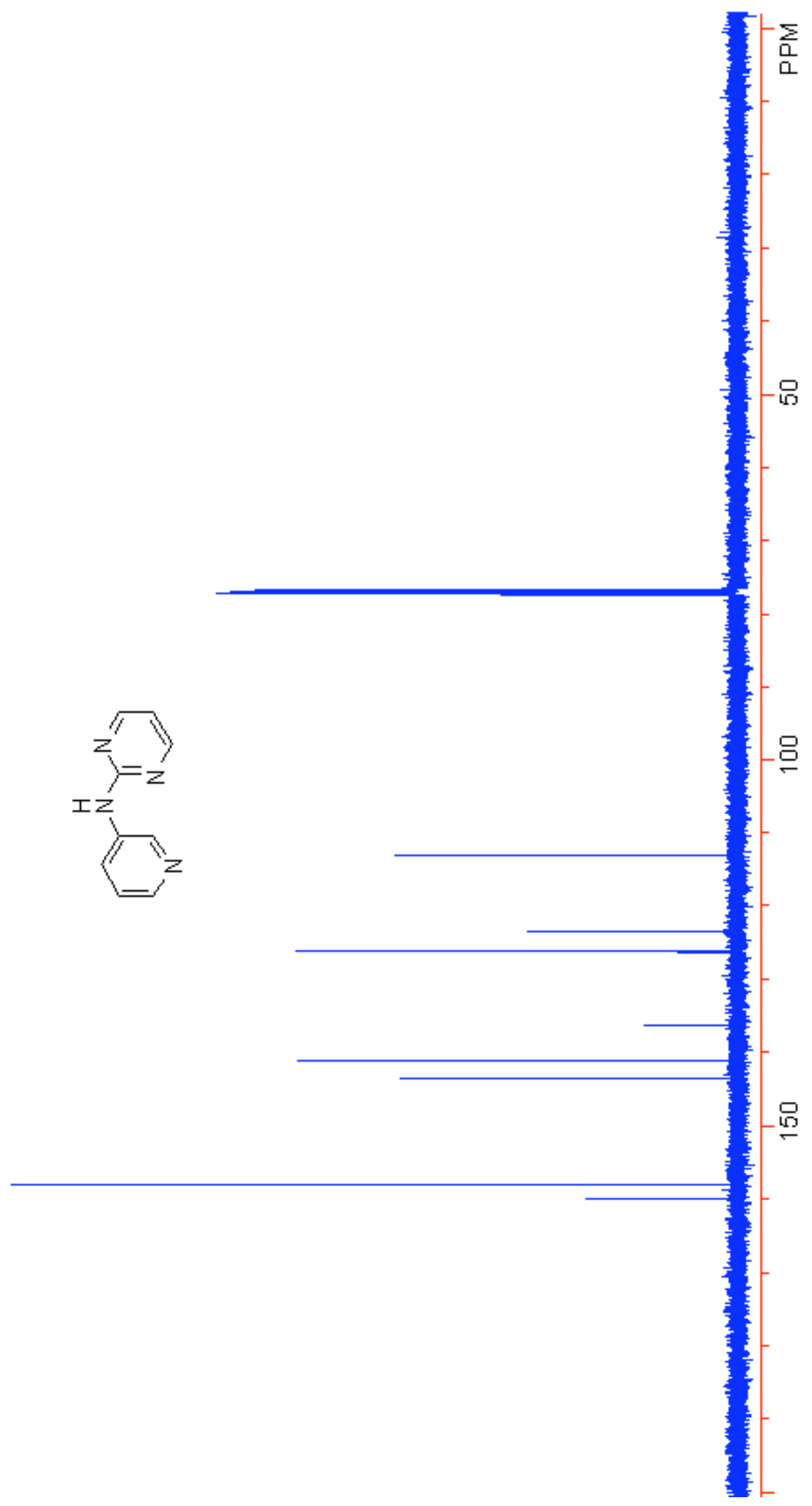




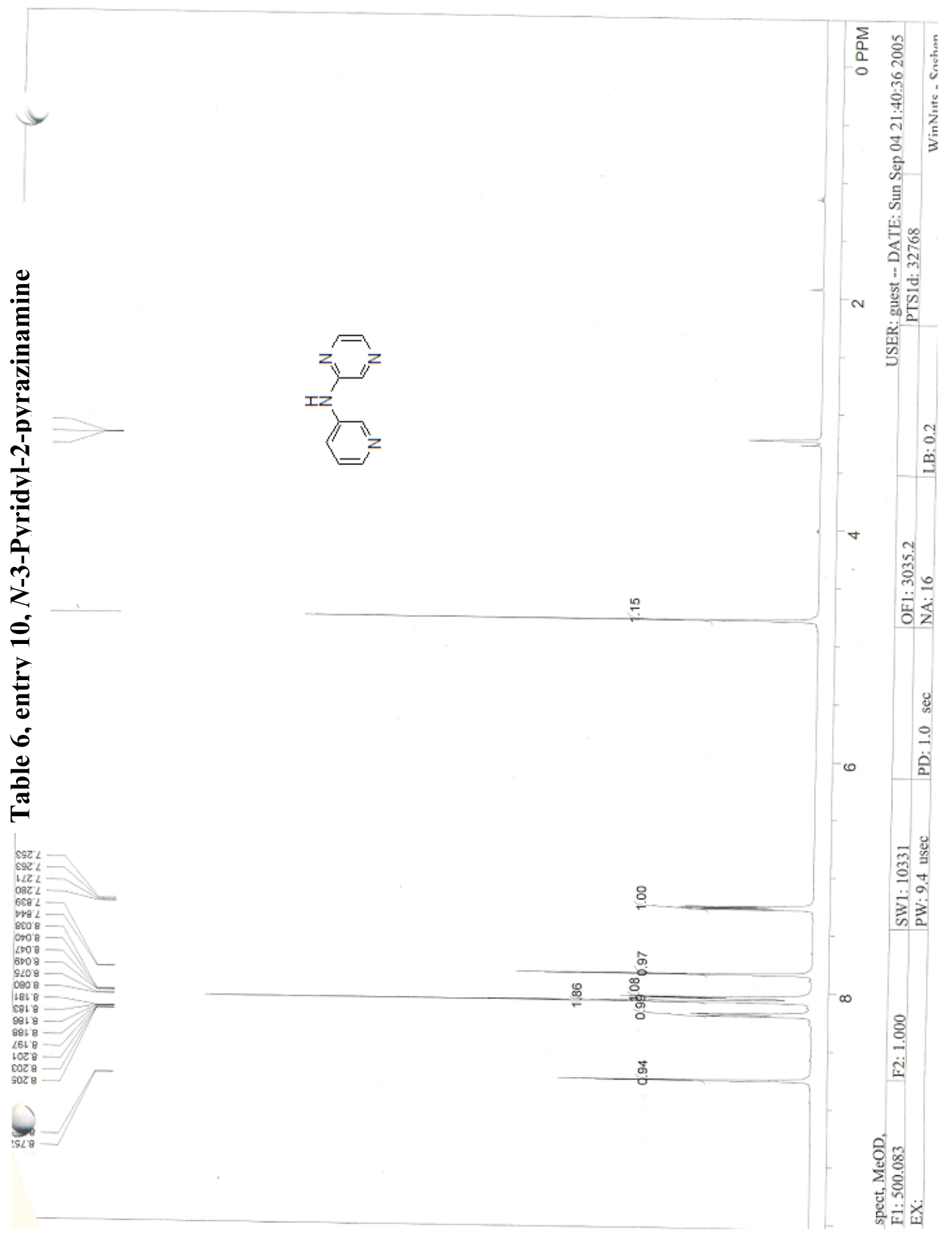




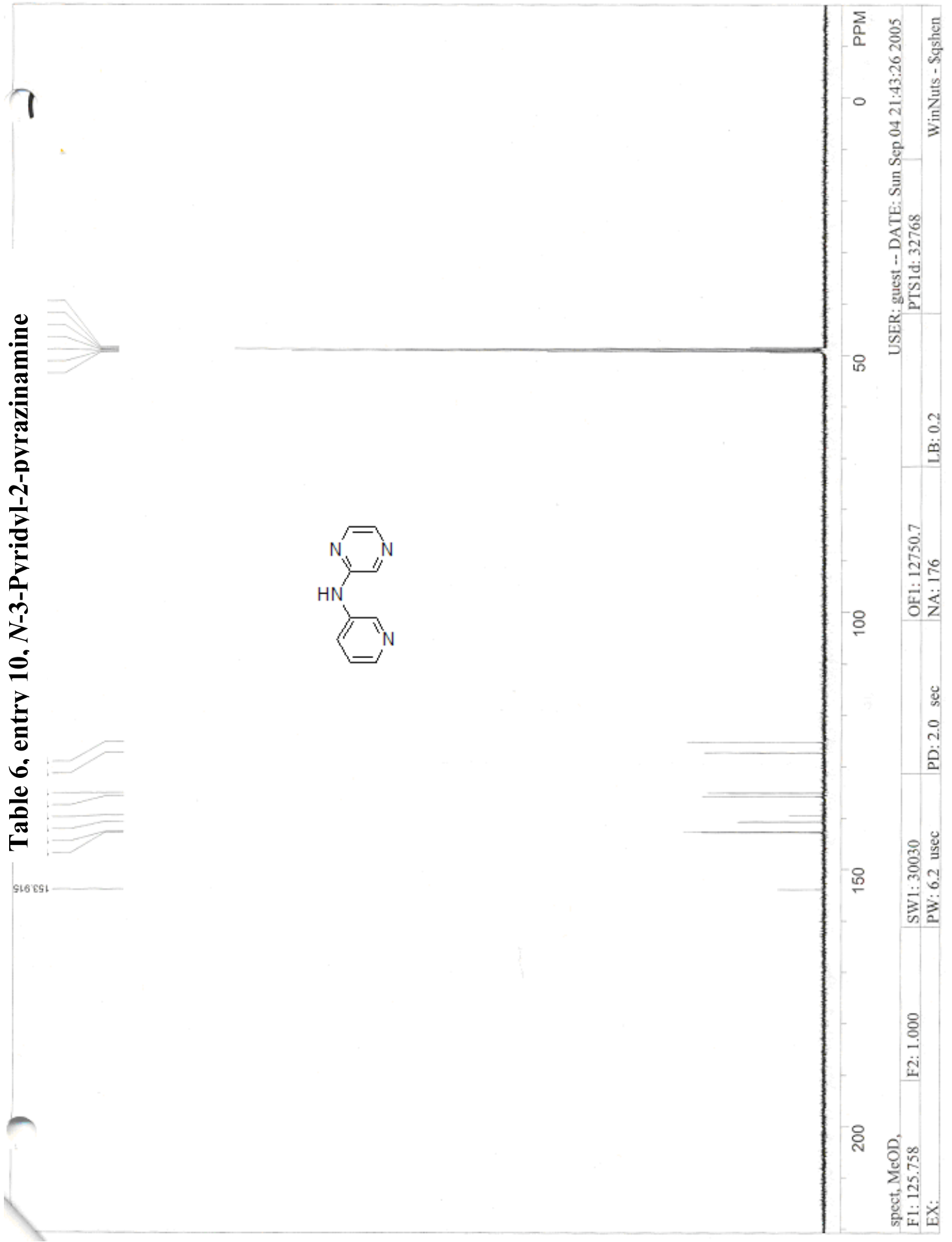




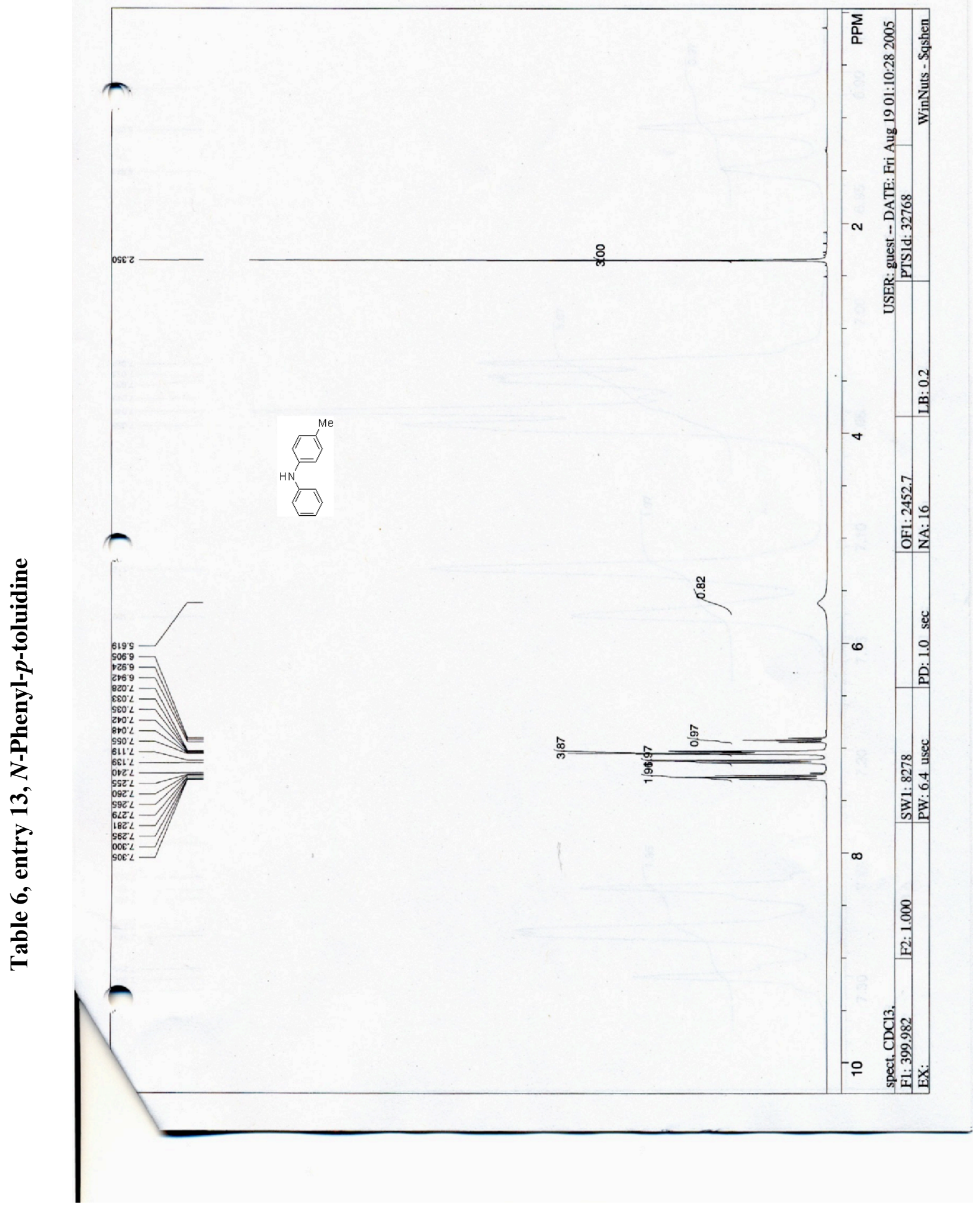




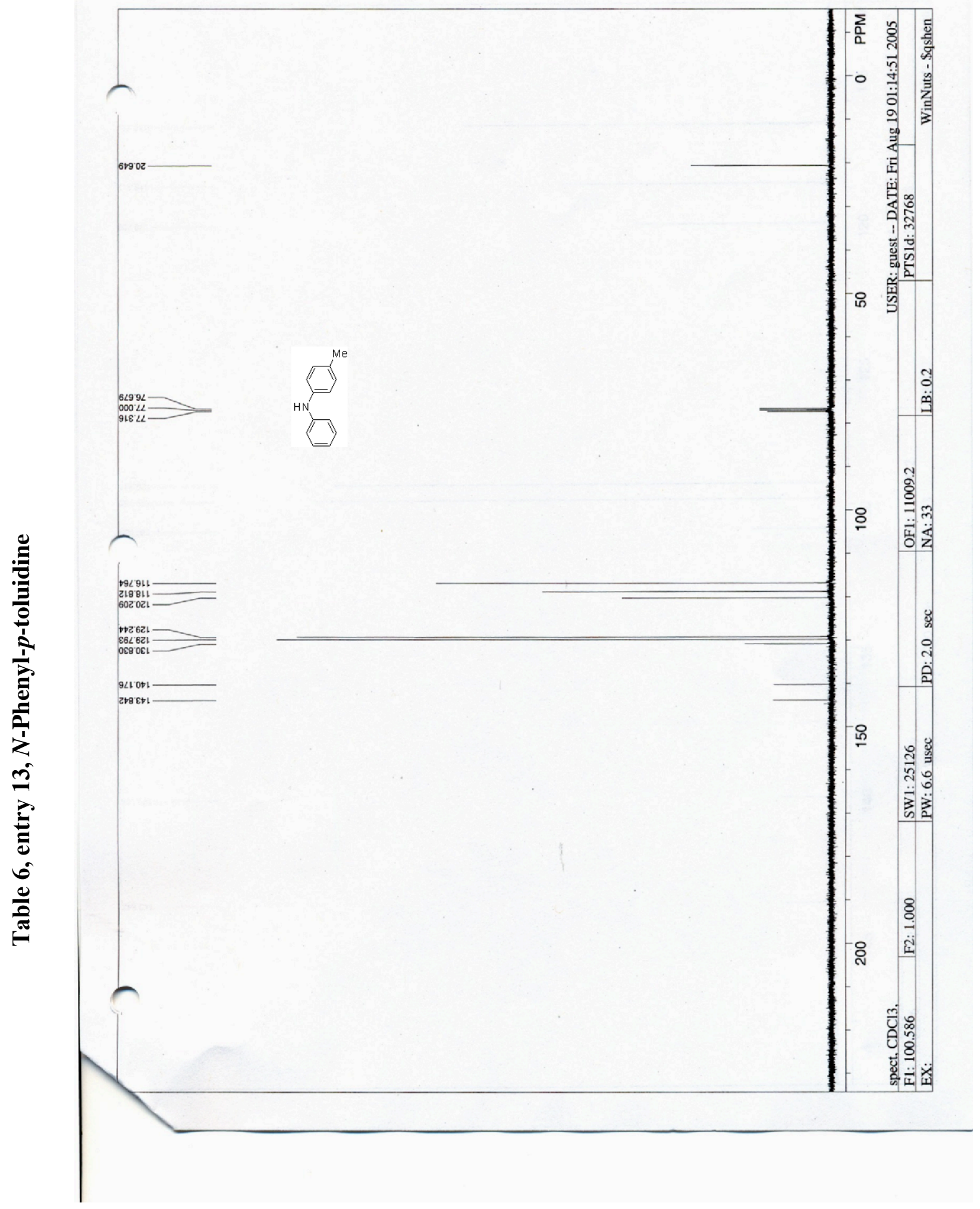




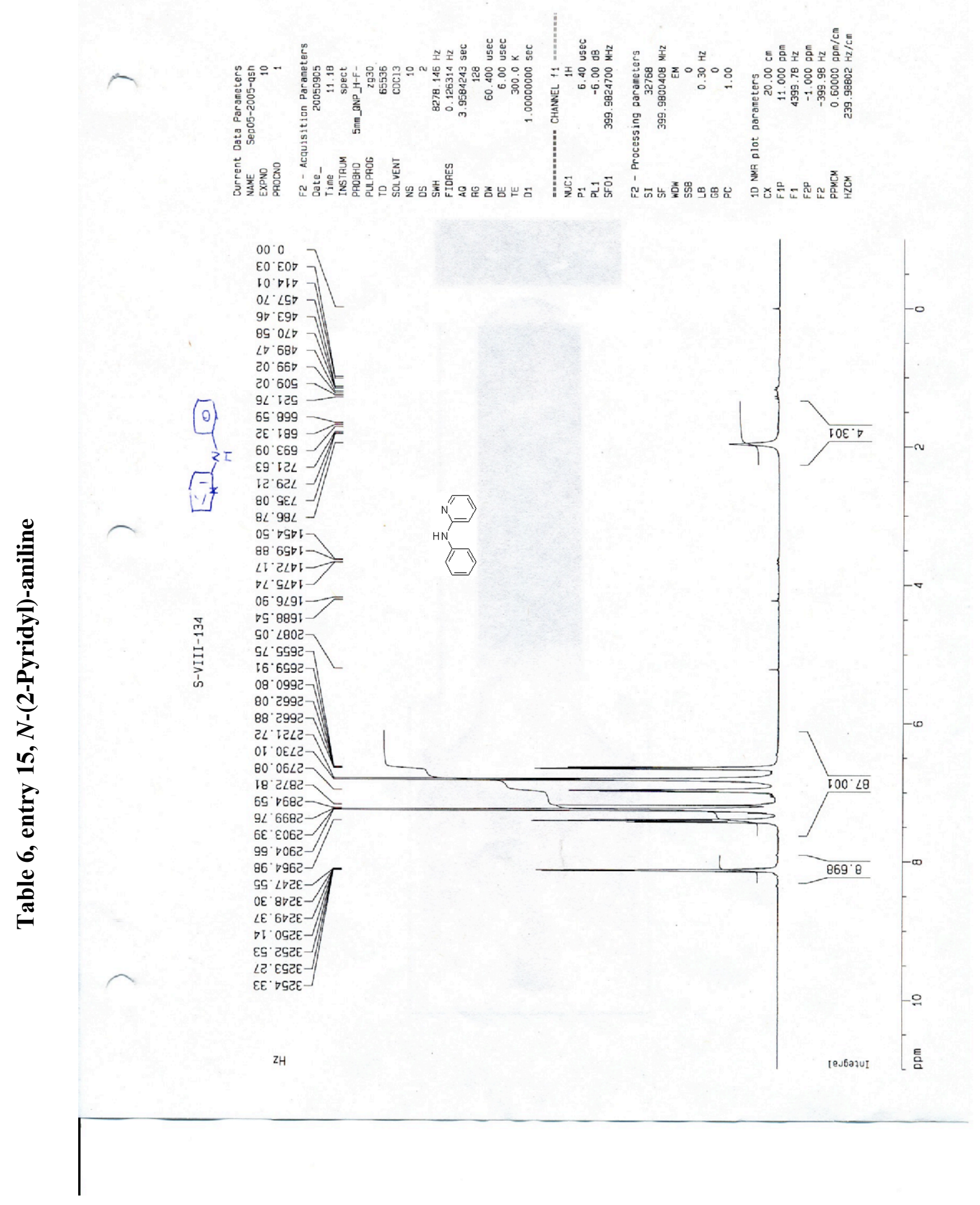




$$
19
$$



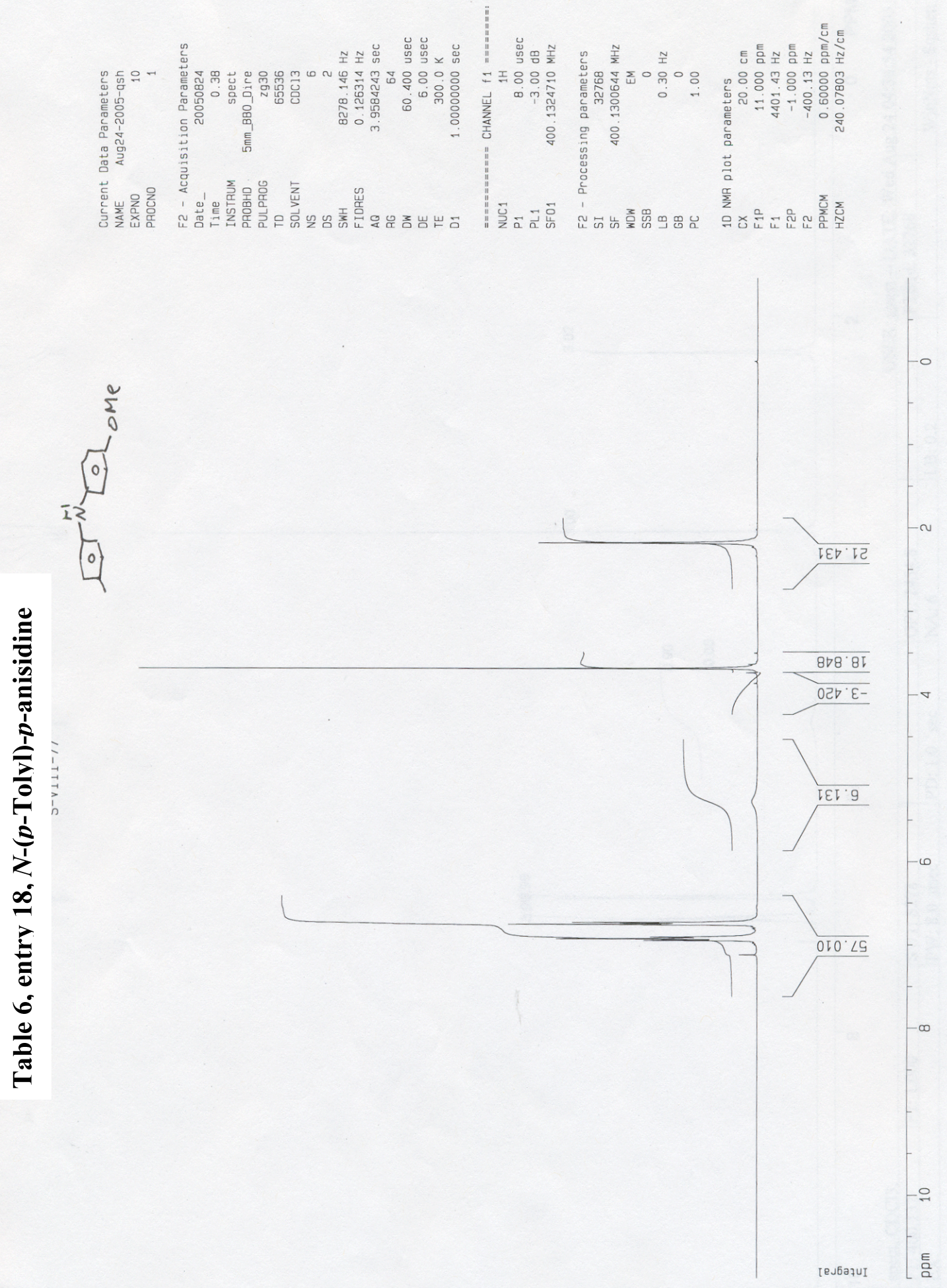

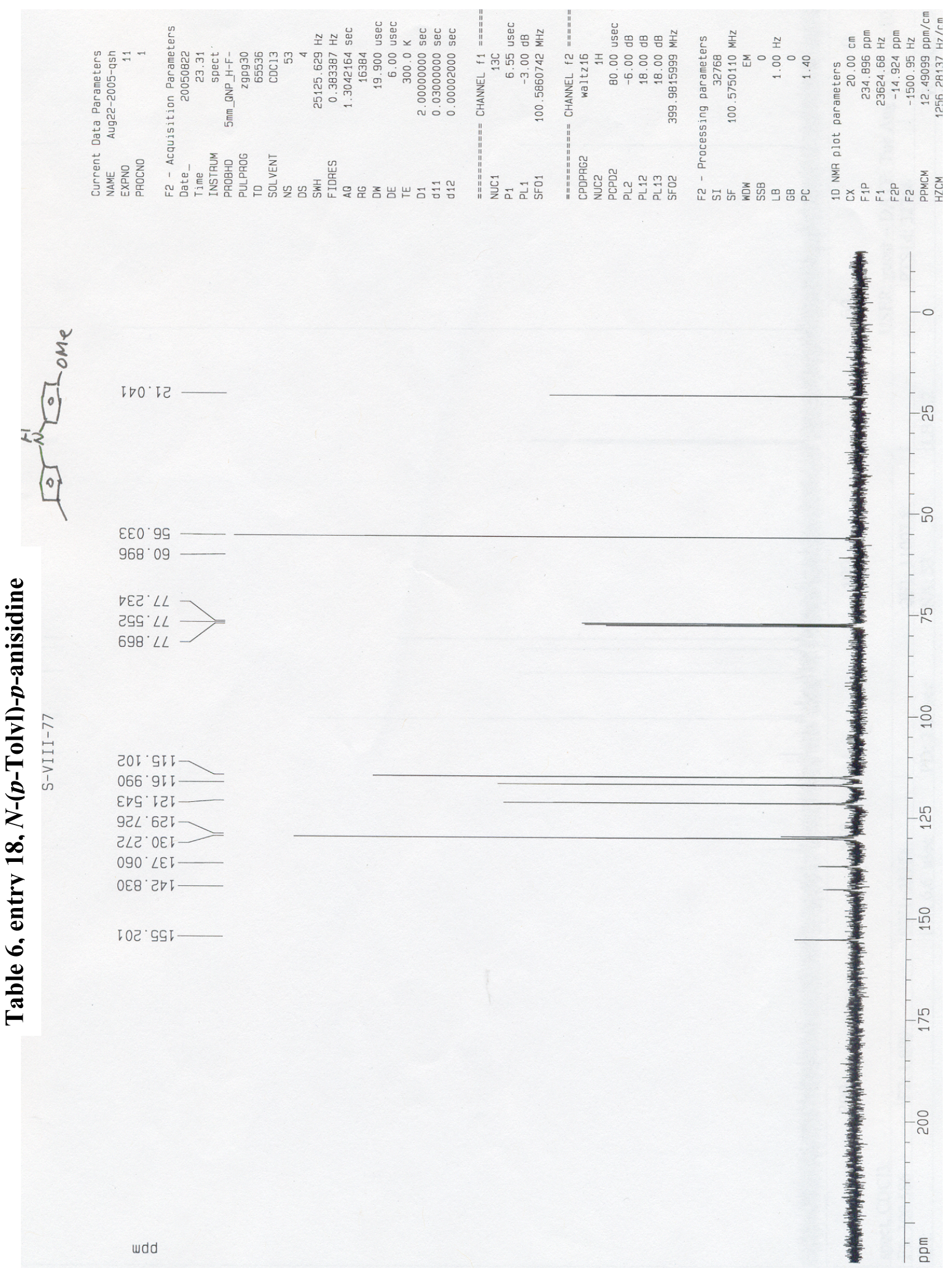


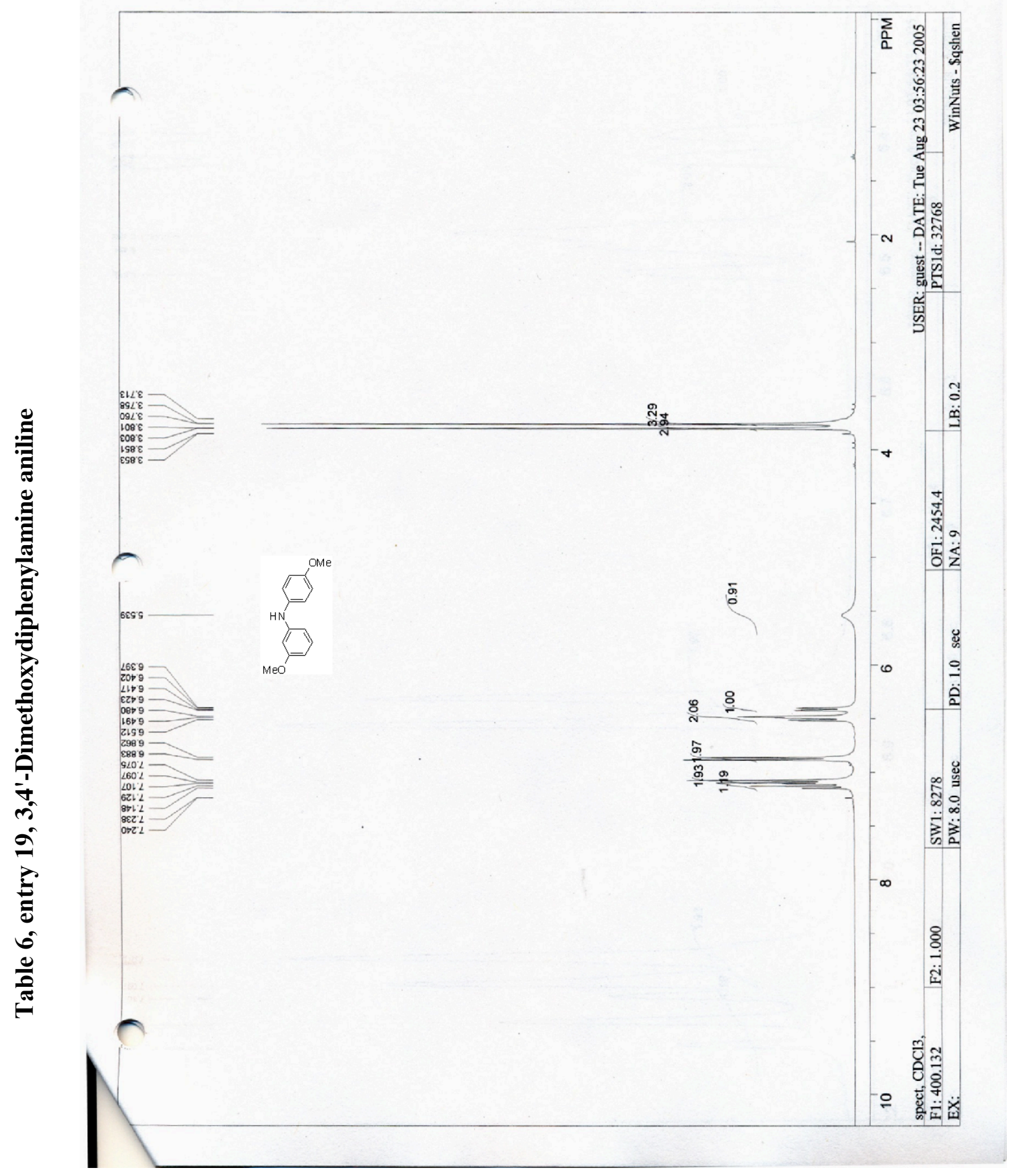




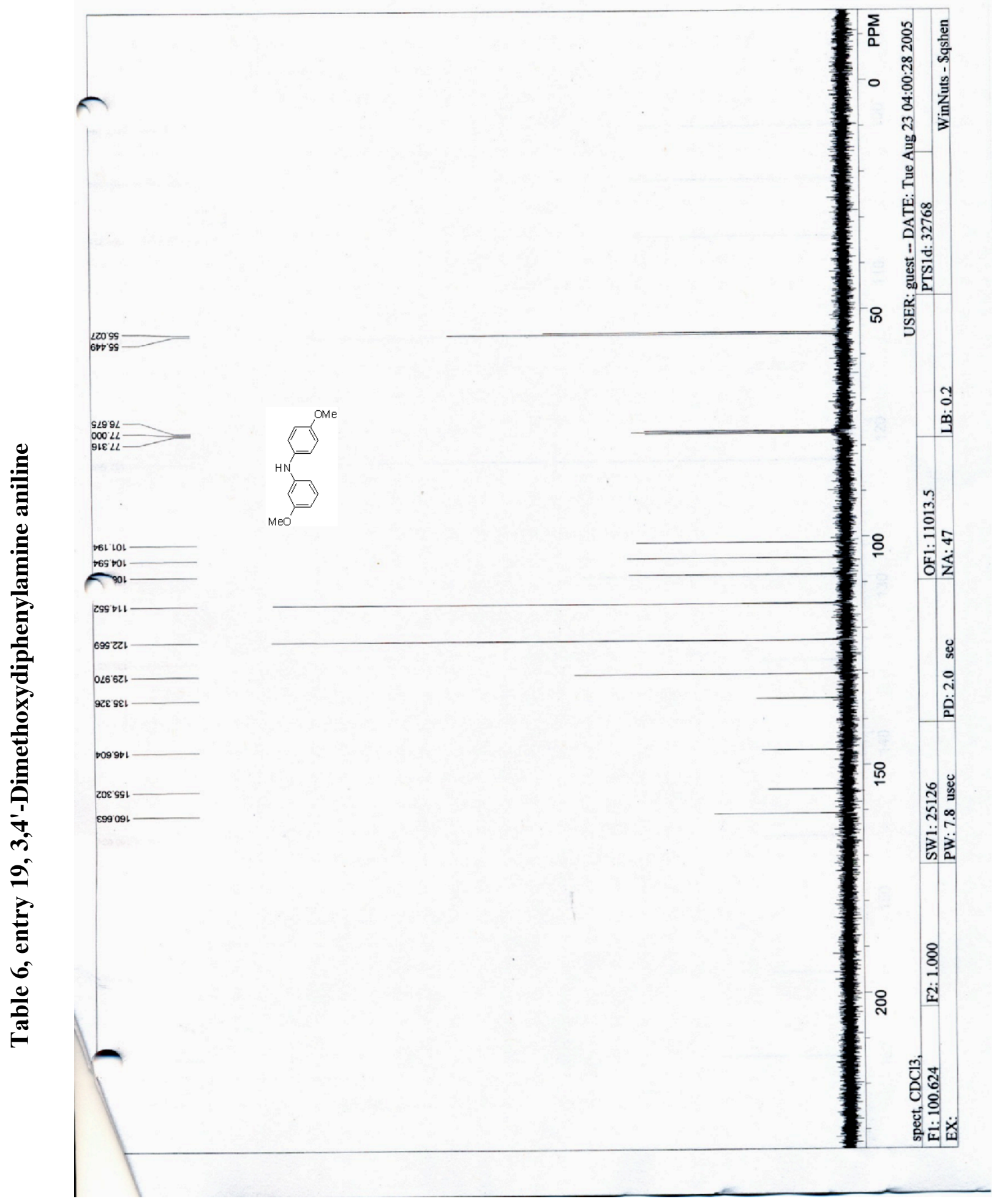




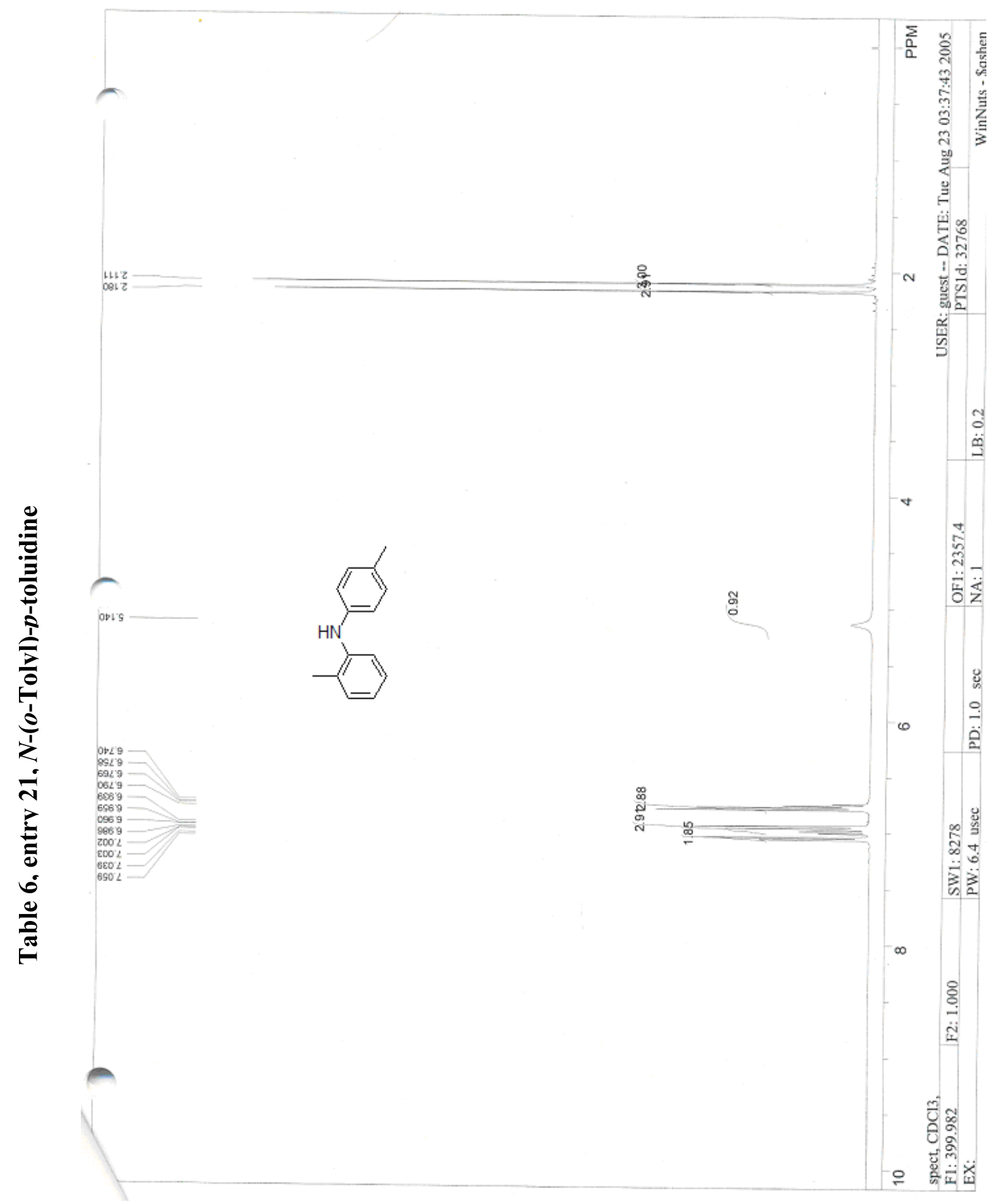




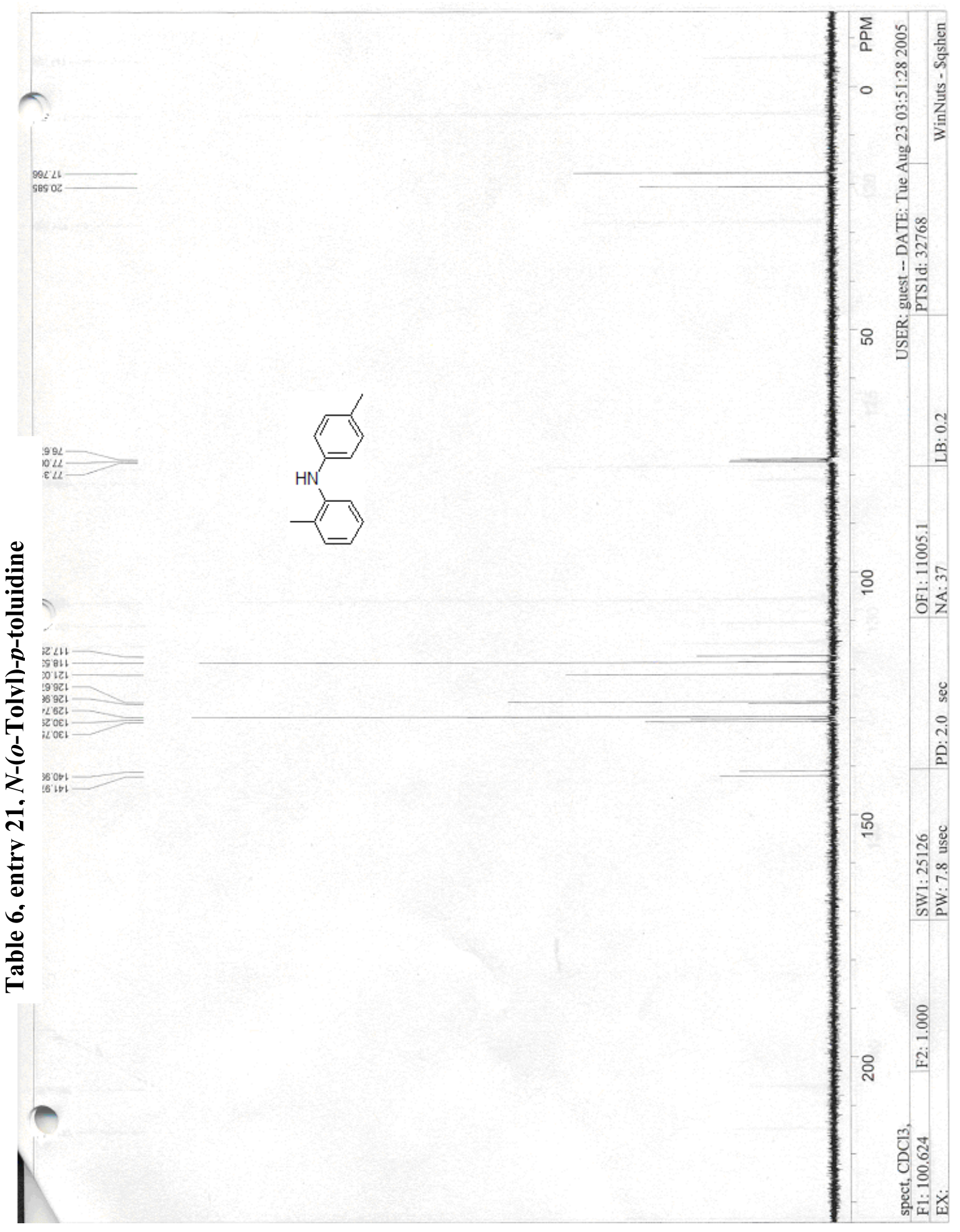




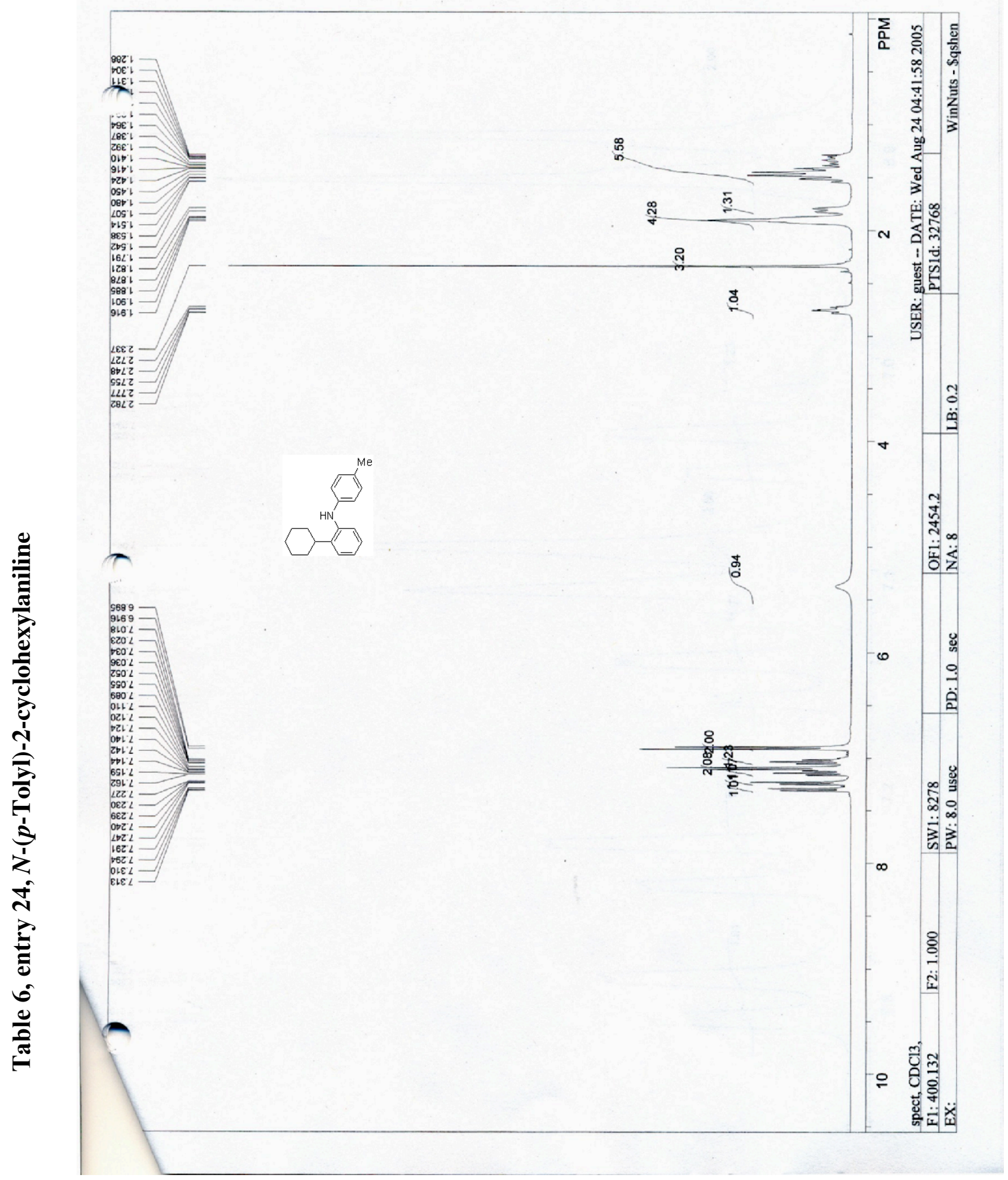




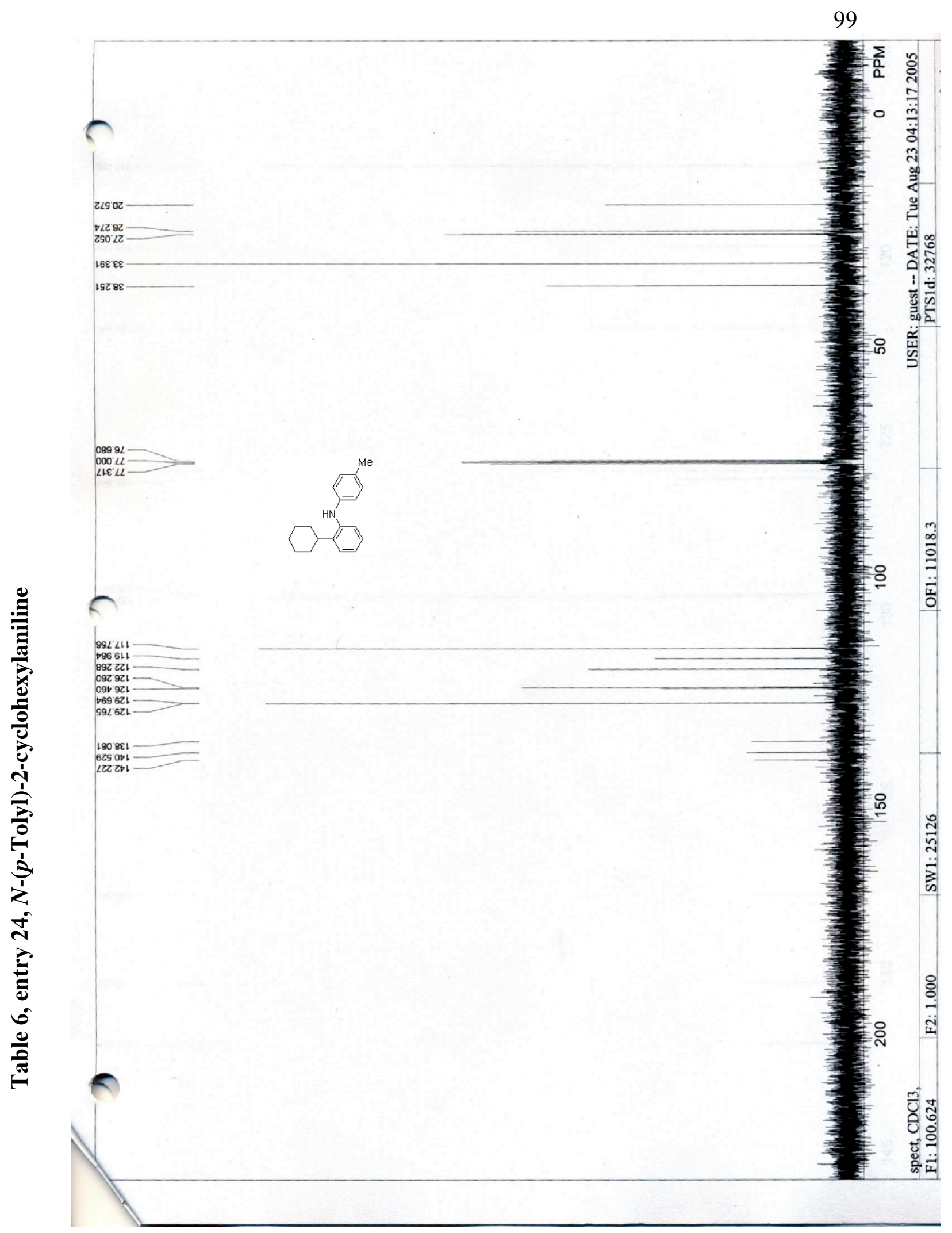




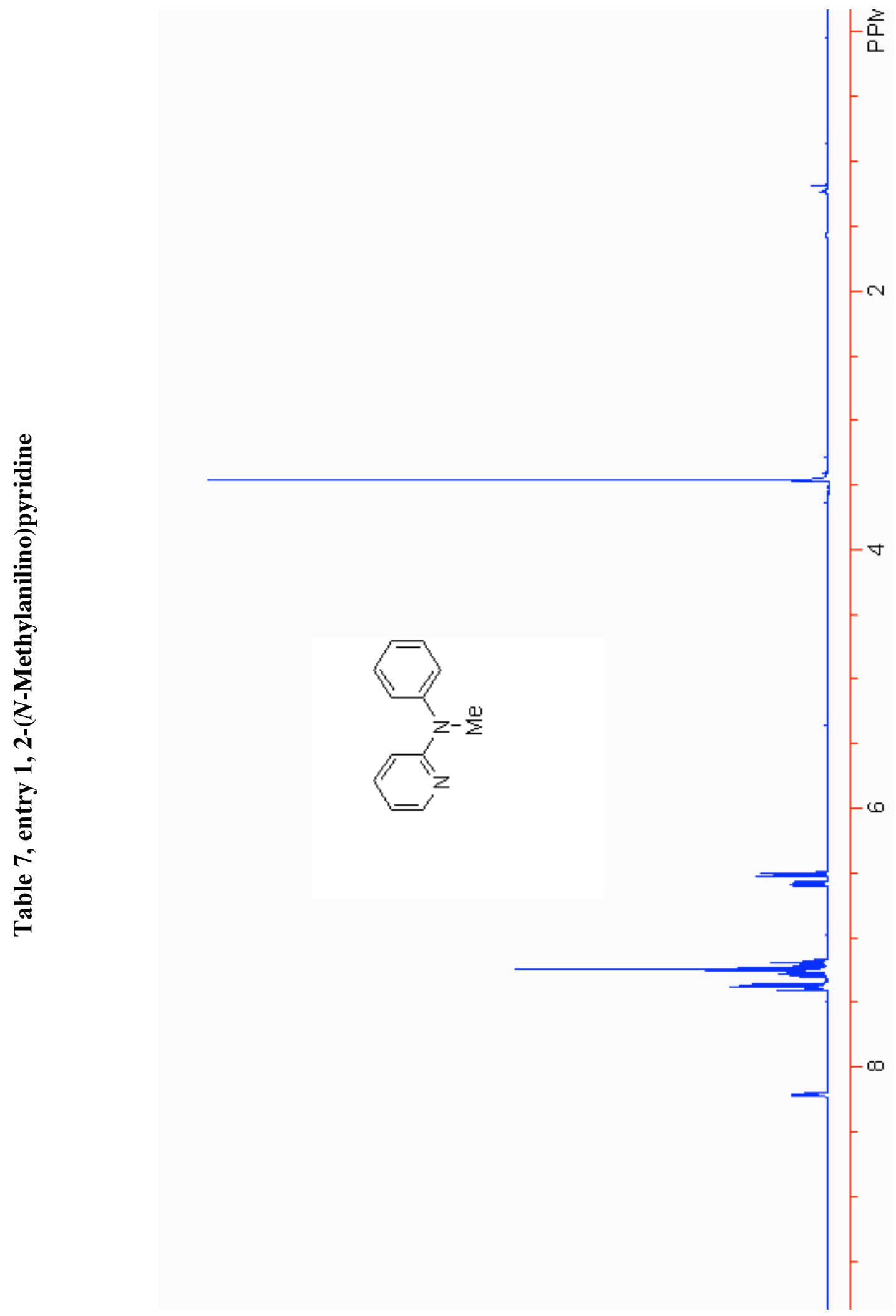


ב⿱艹

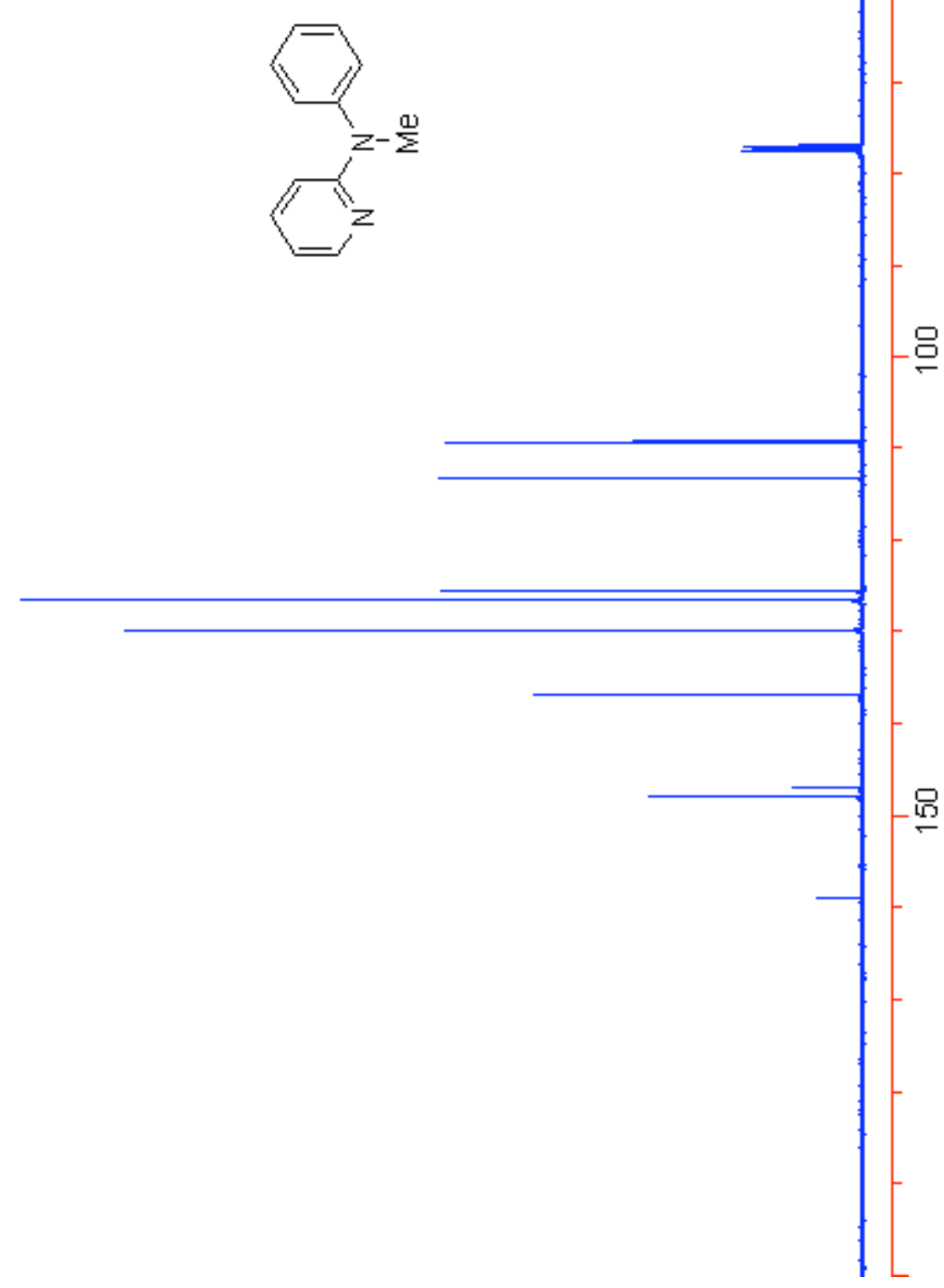

$\mid 5$ 

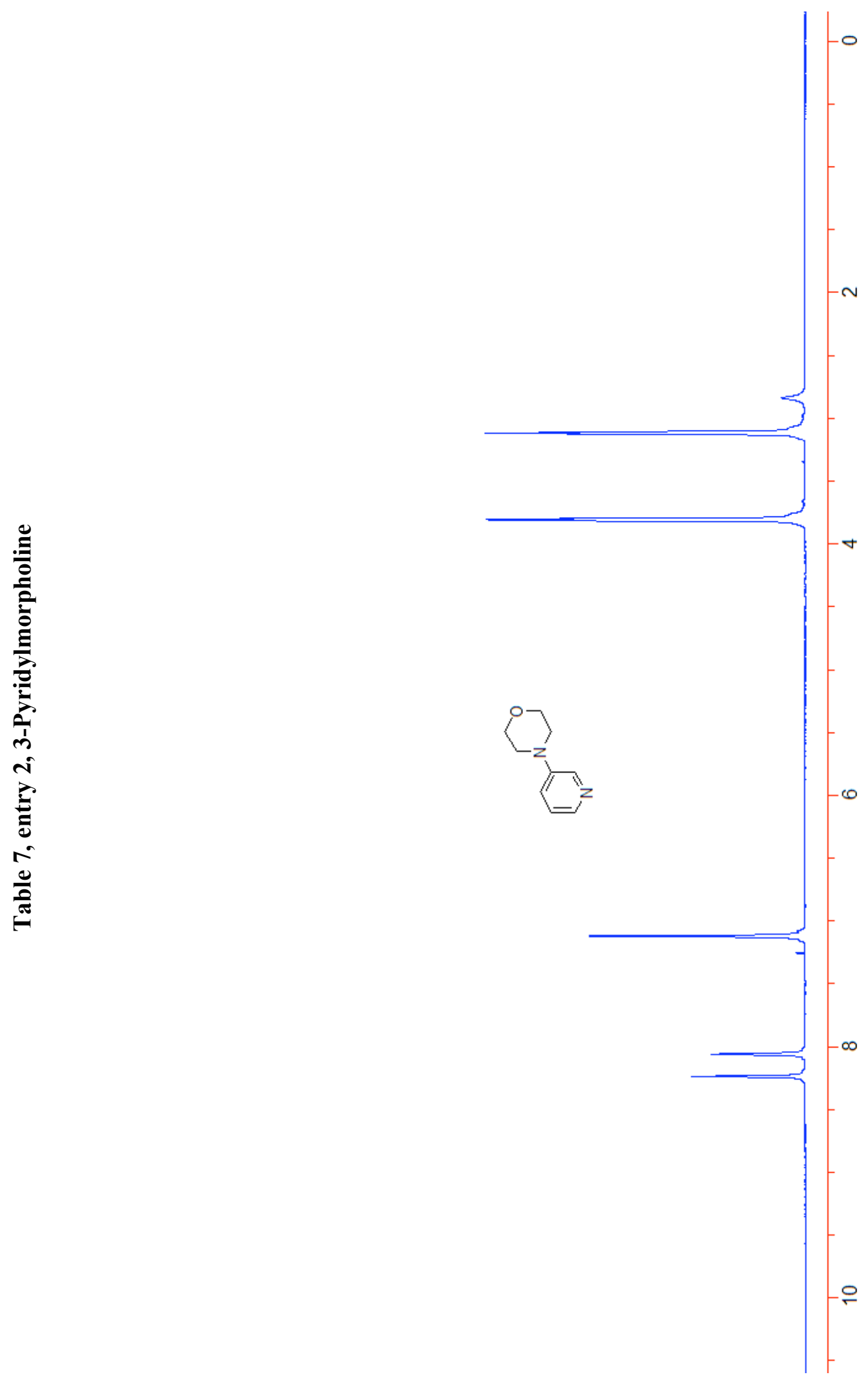
है.

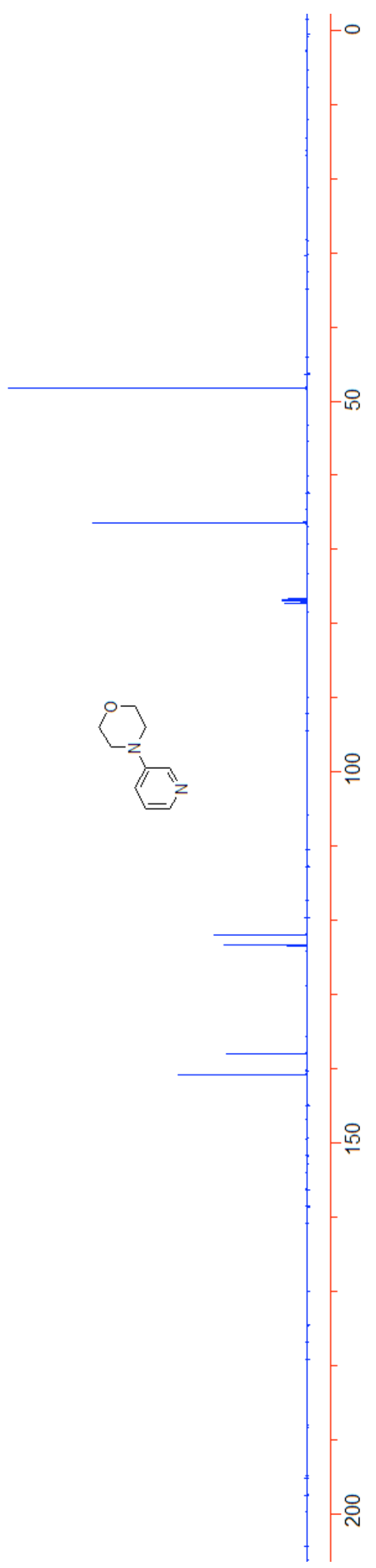



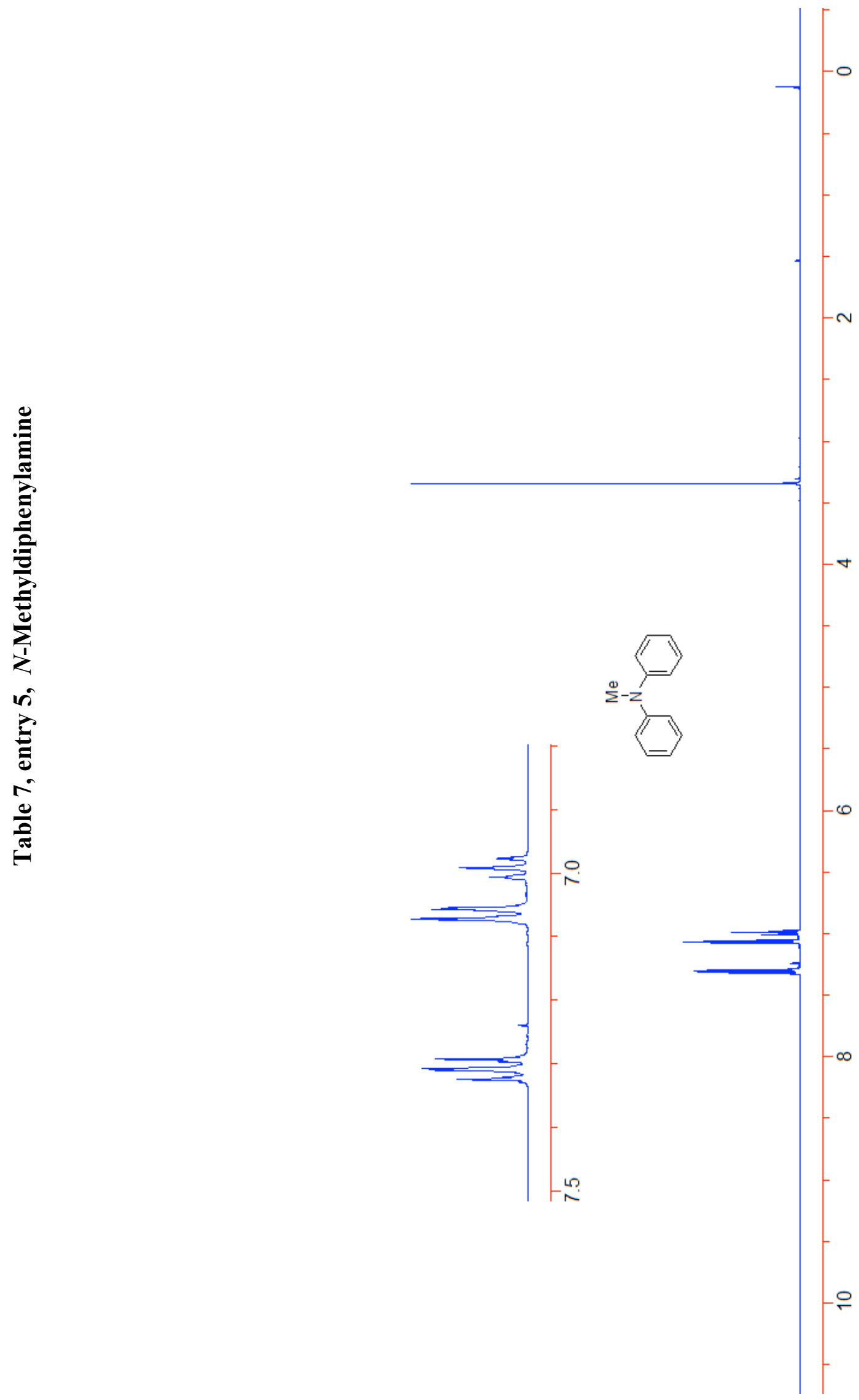
है

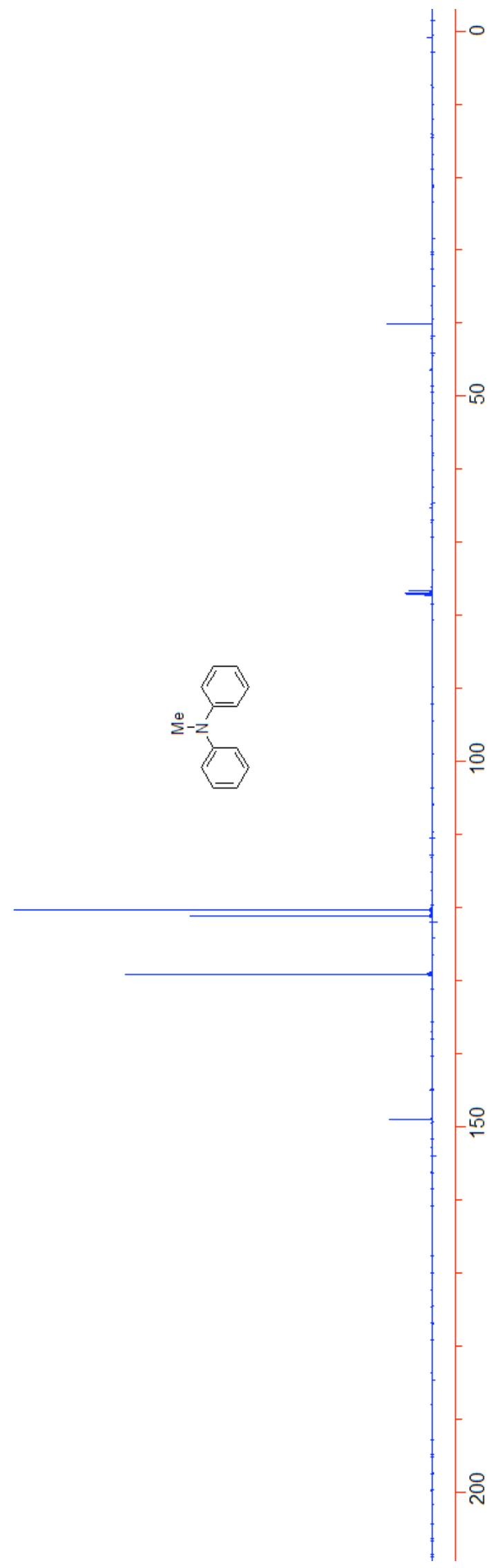



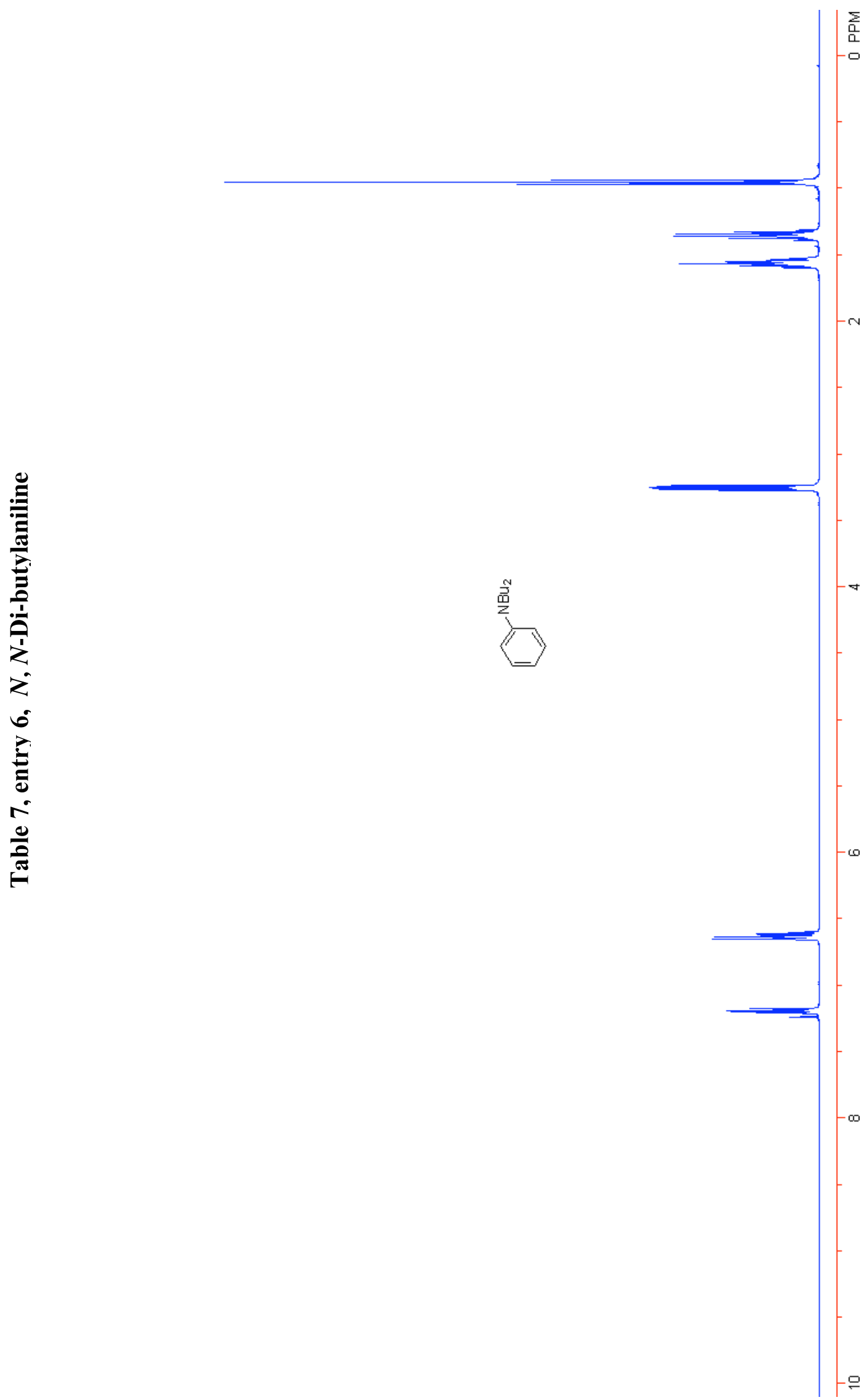
之े

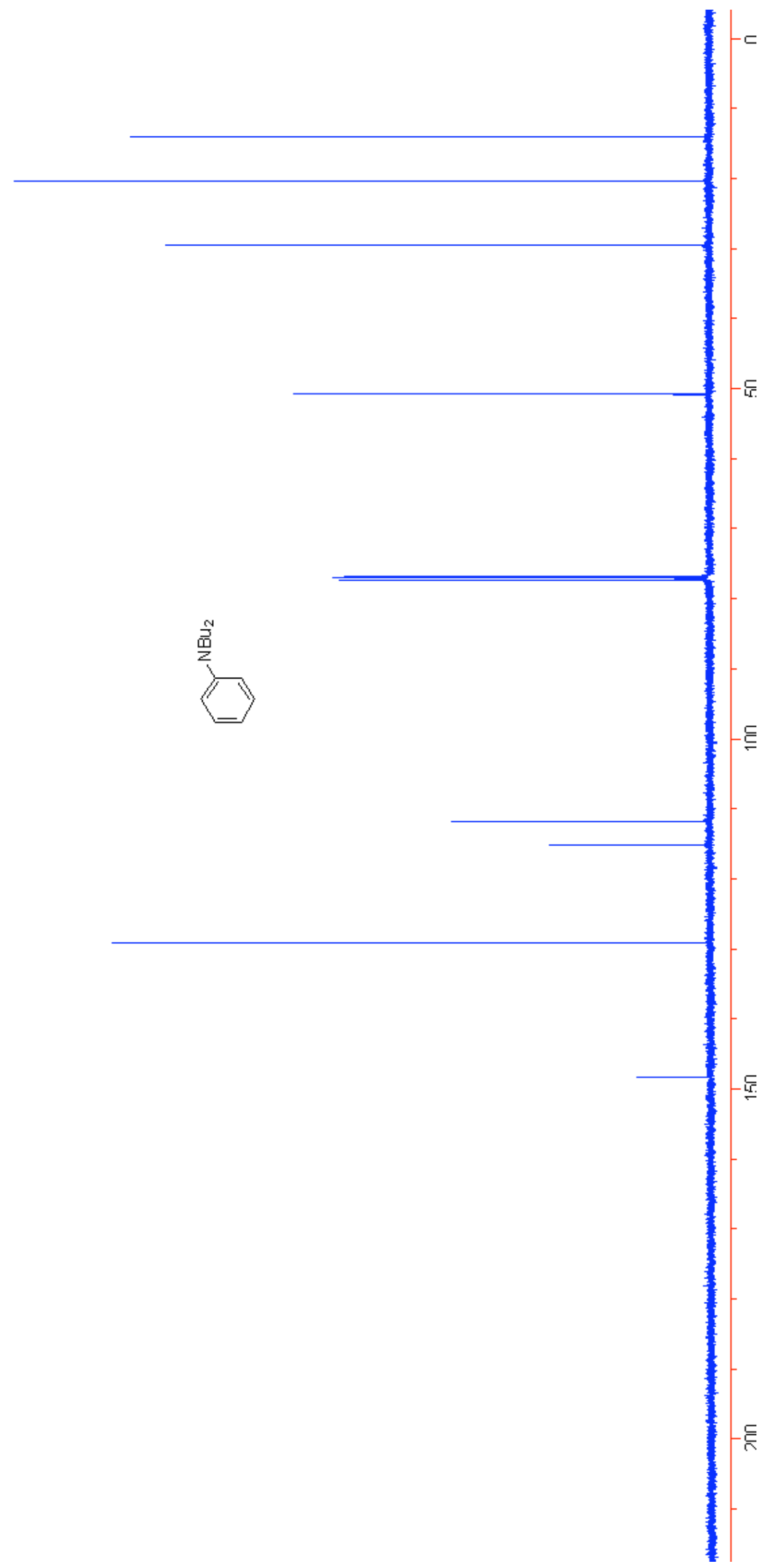


है.

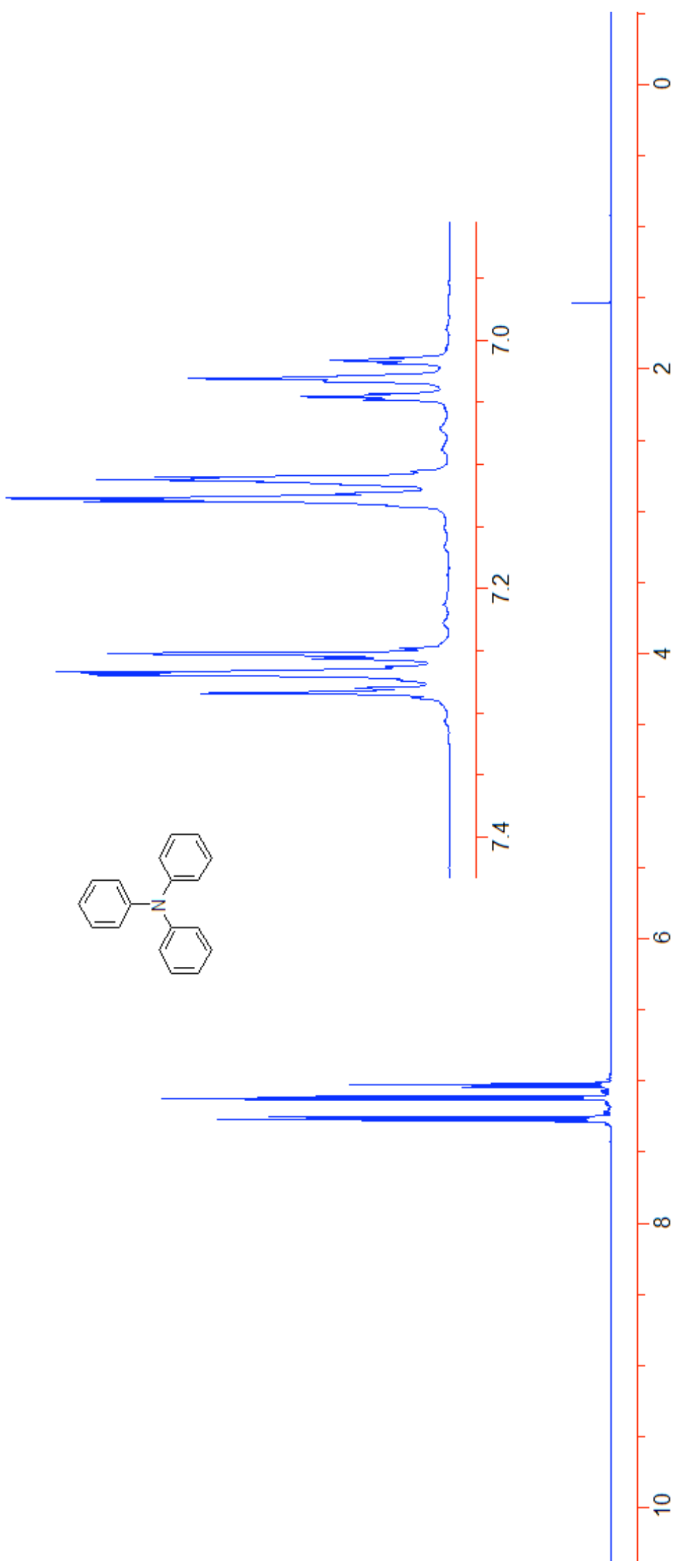


है.

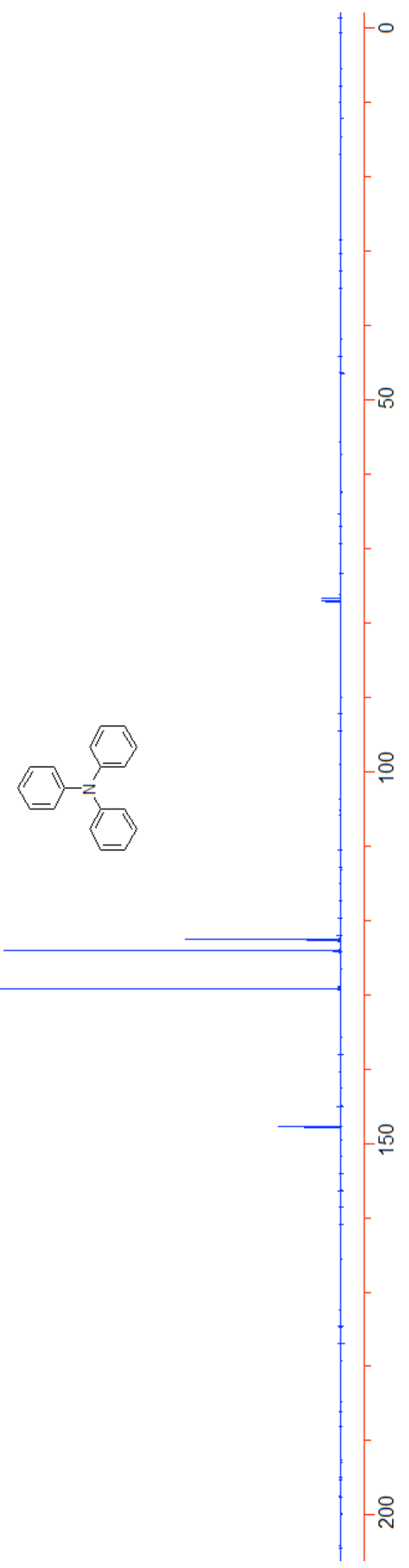


量

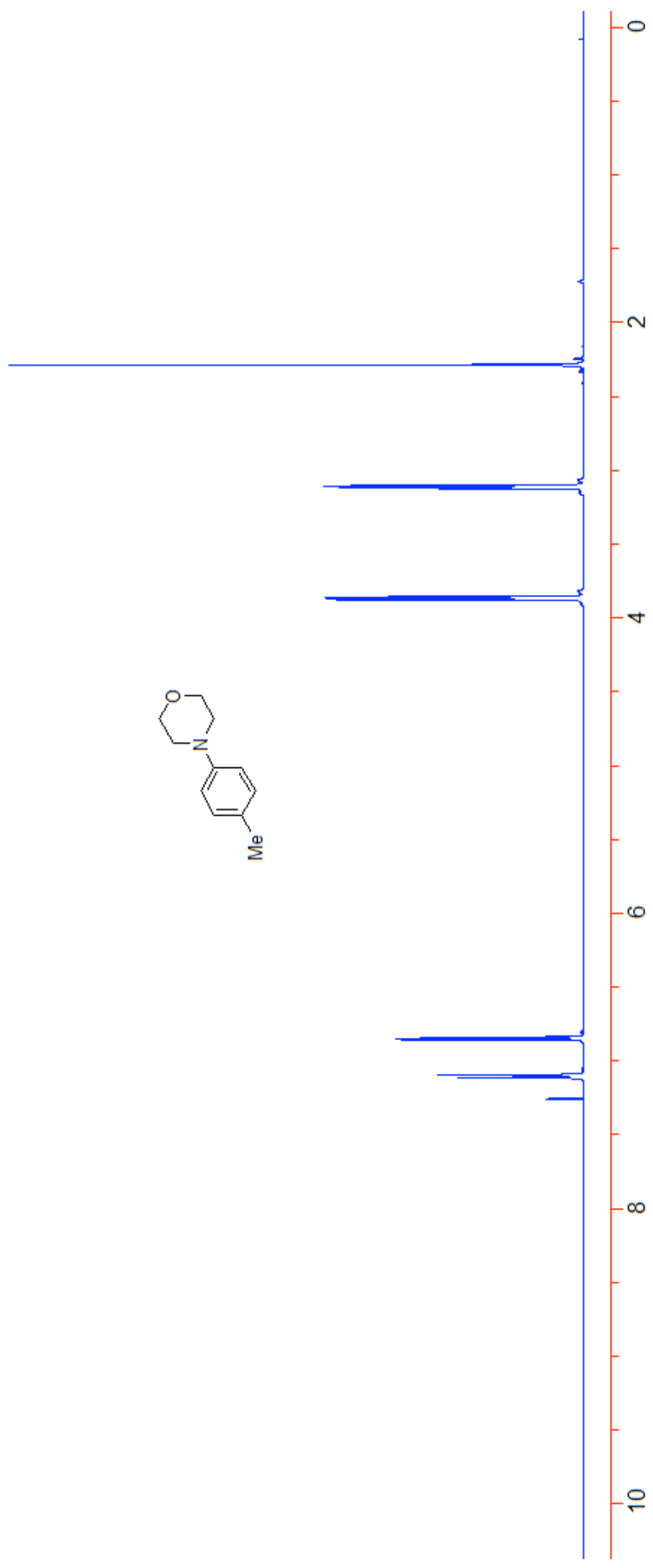




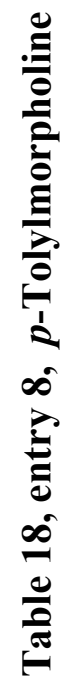

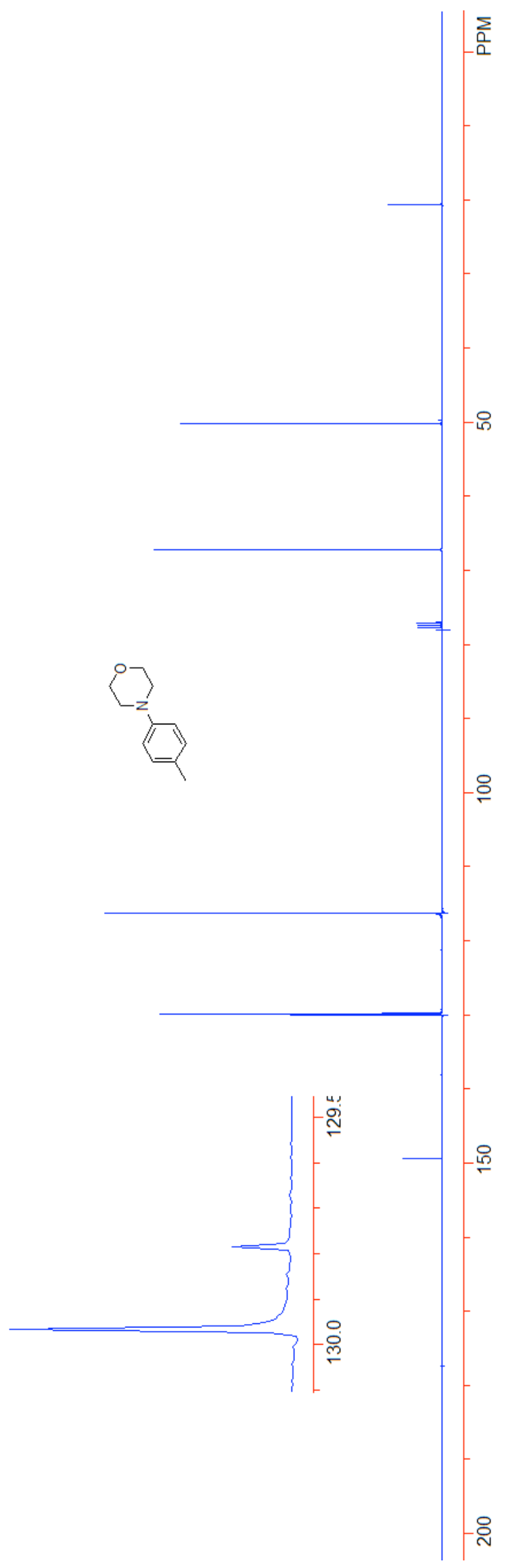



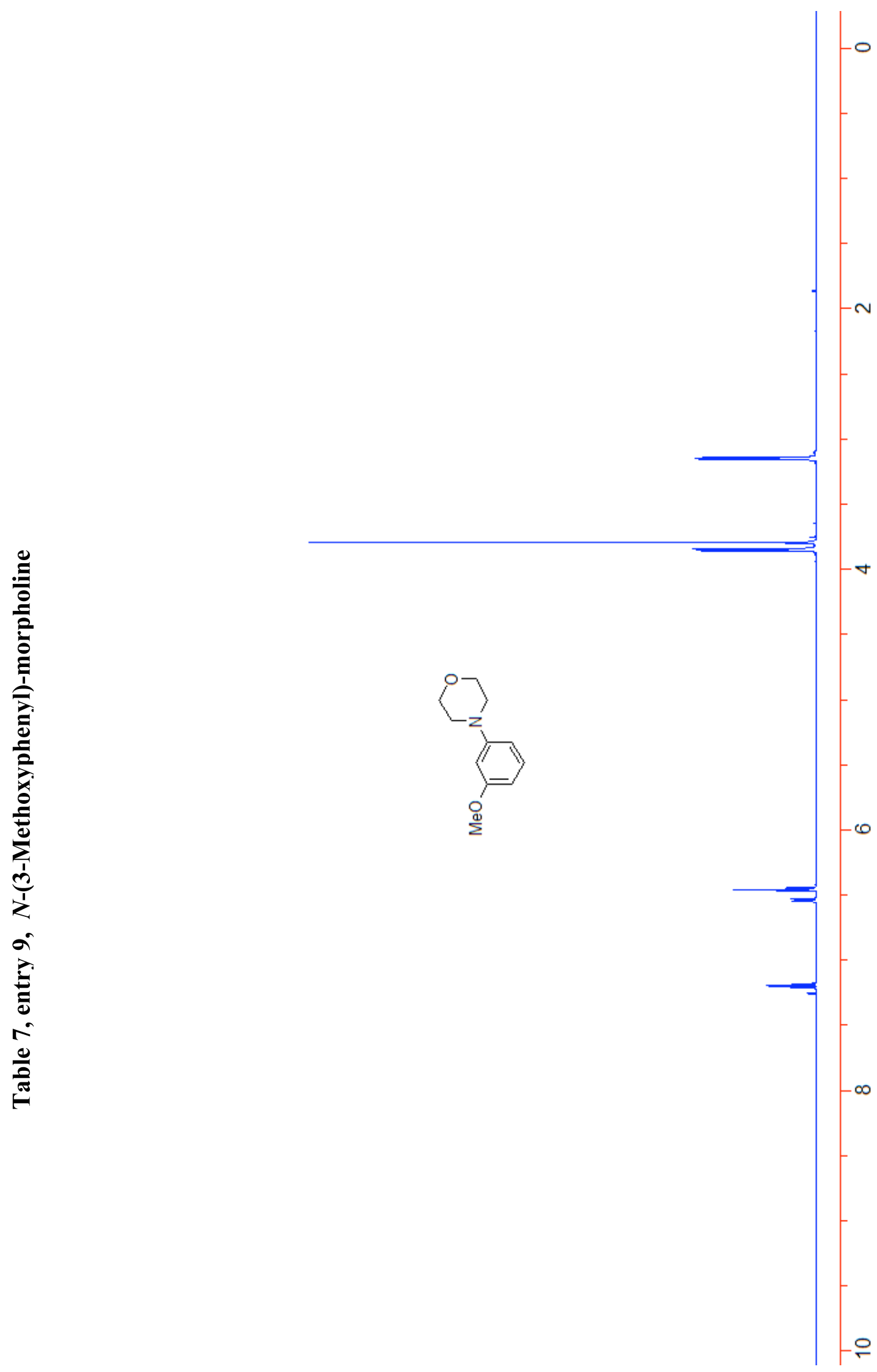
है

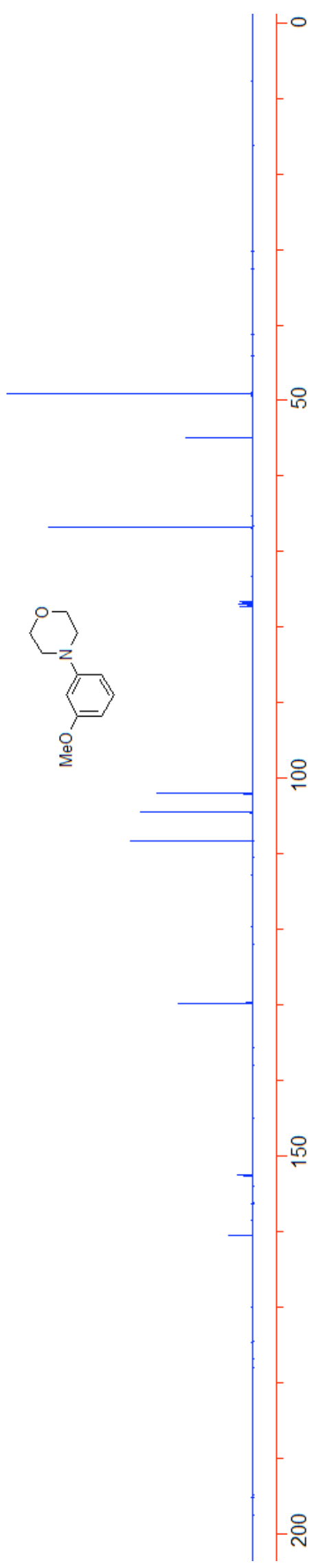




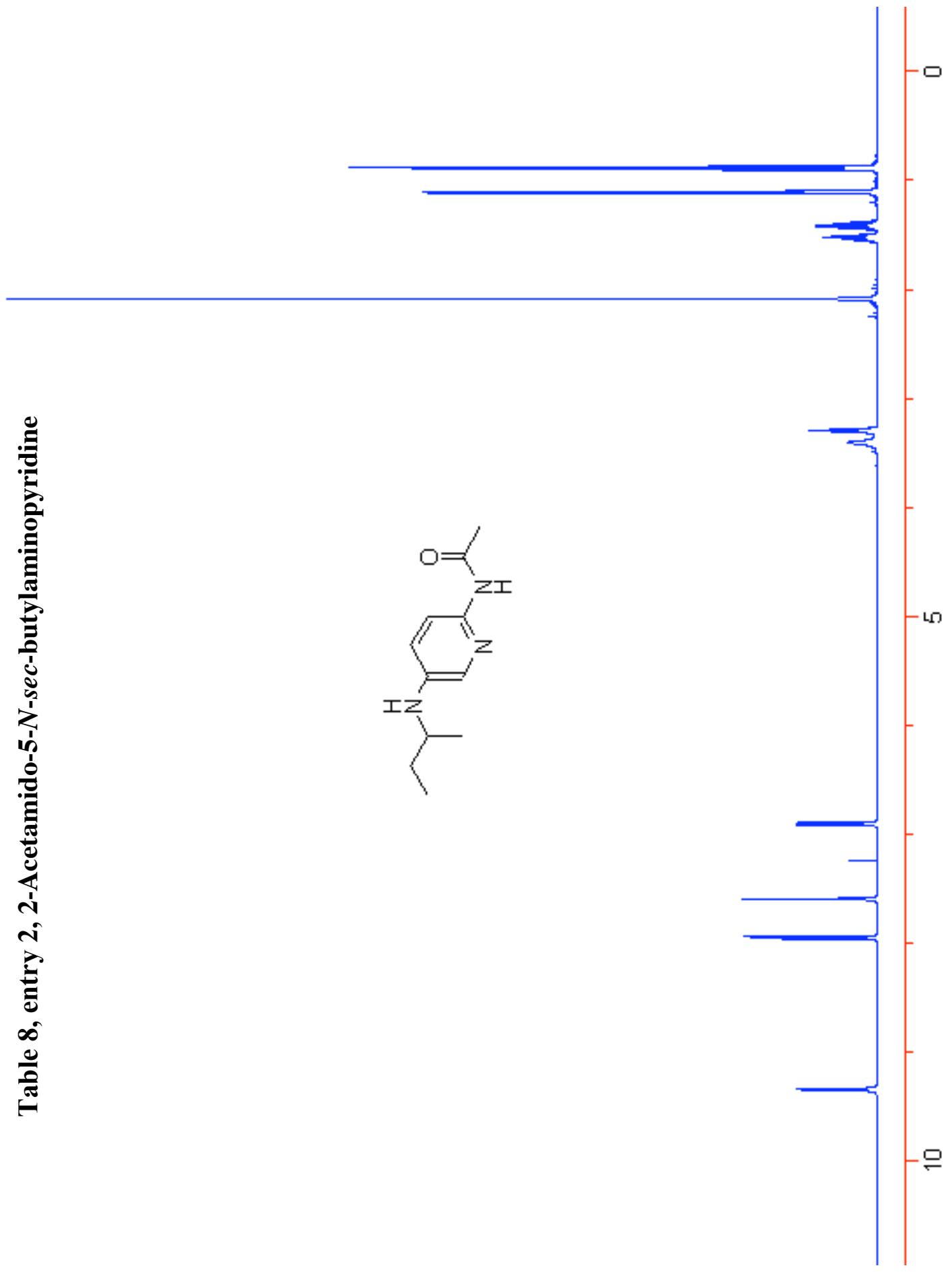




$$
17
$$


'0

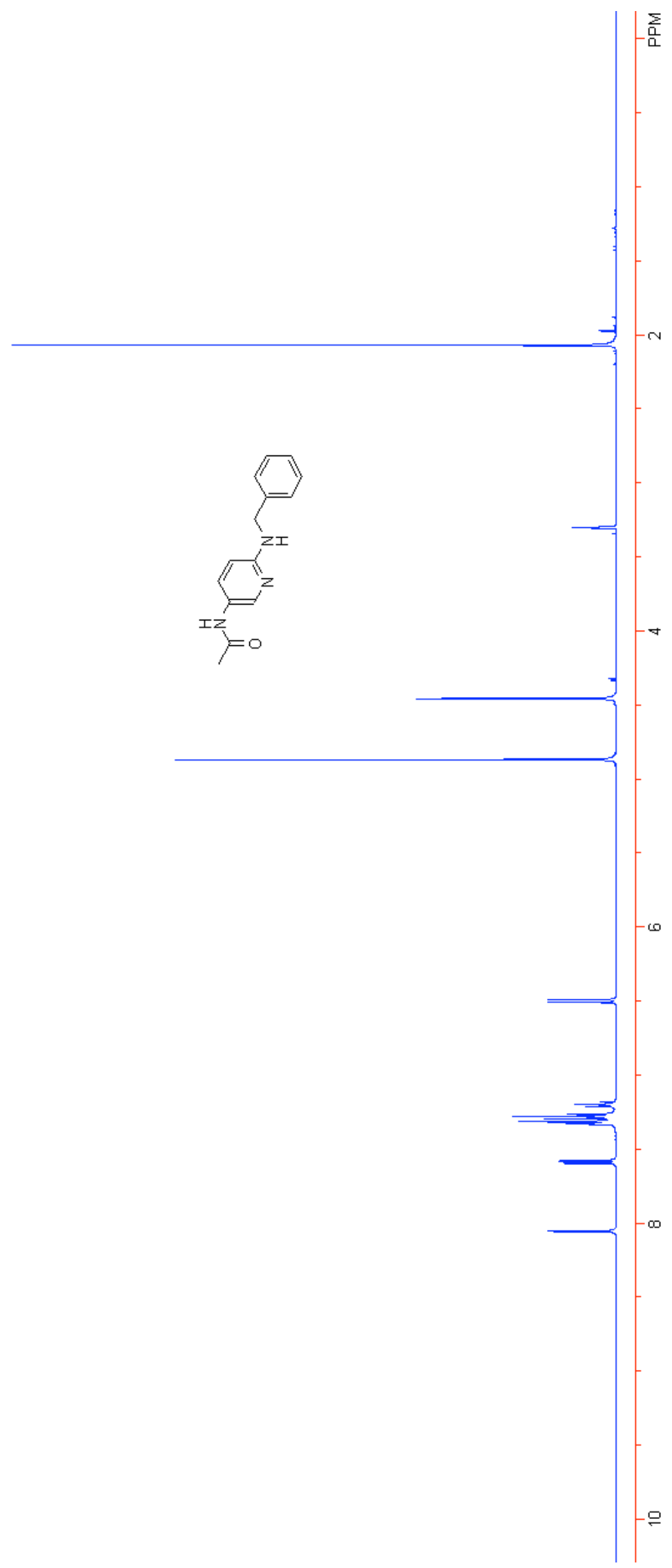




$$
11
$$




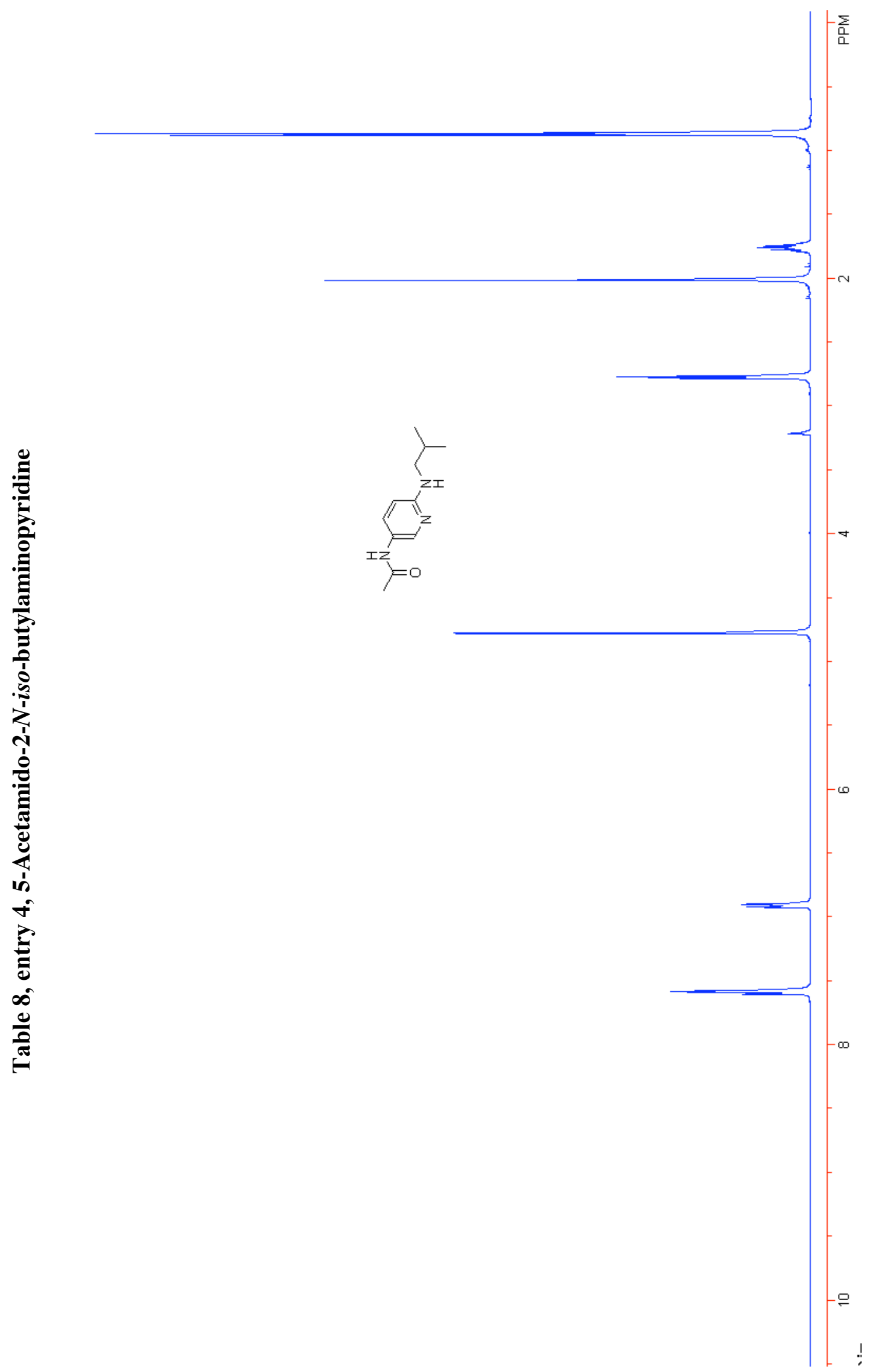




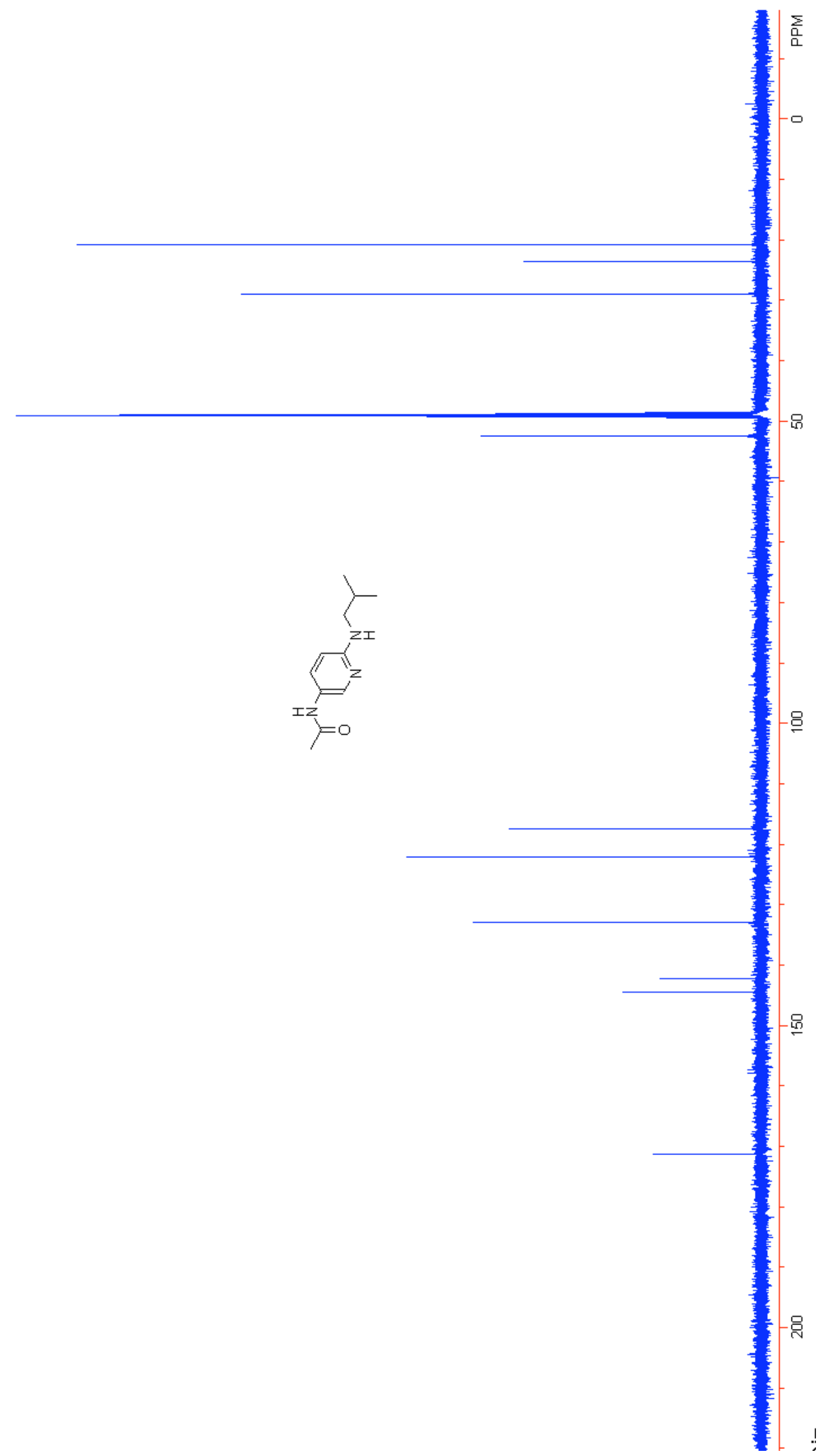




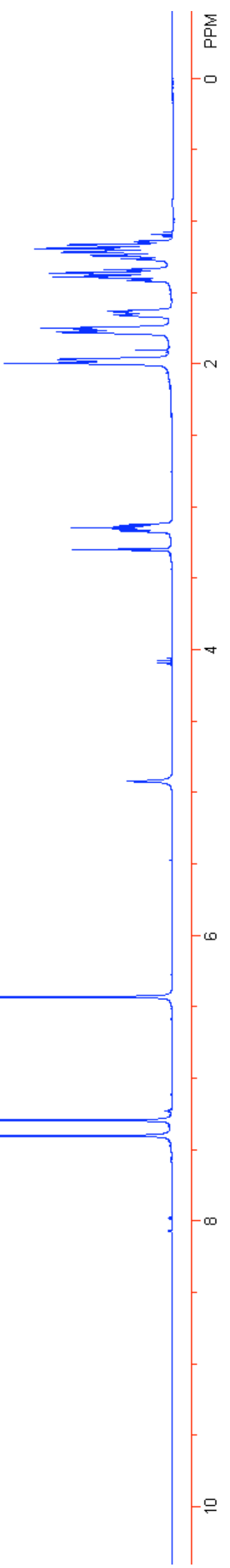


121

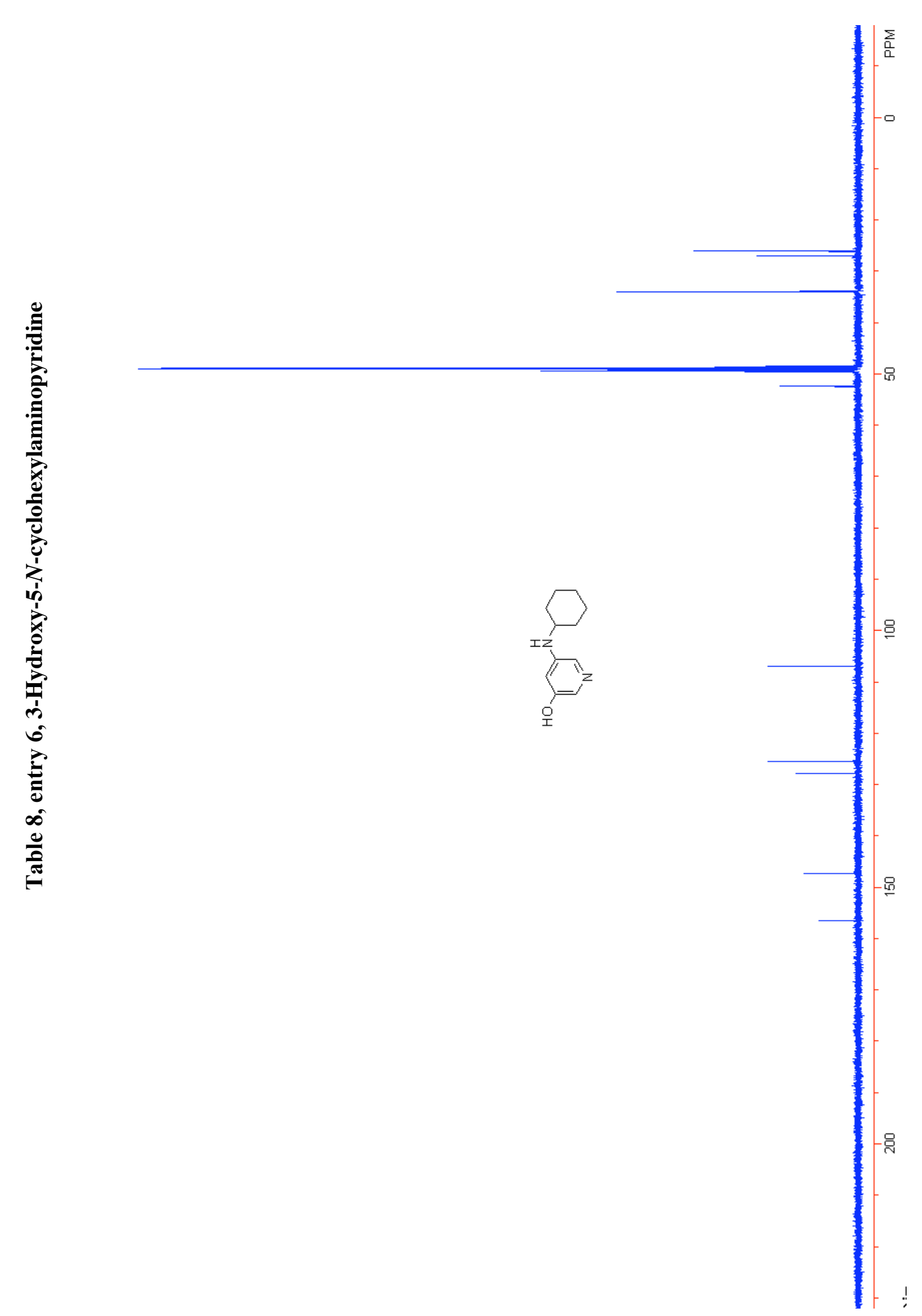



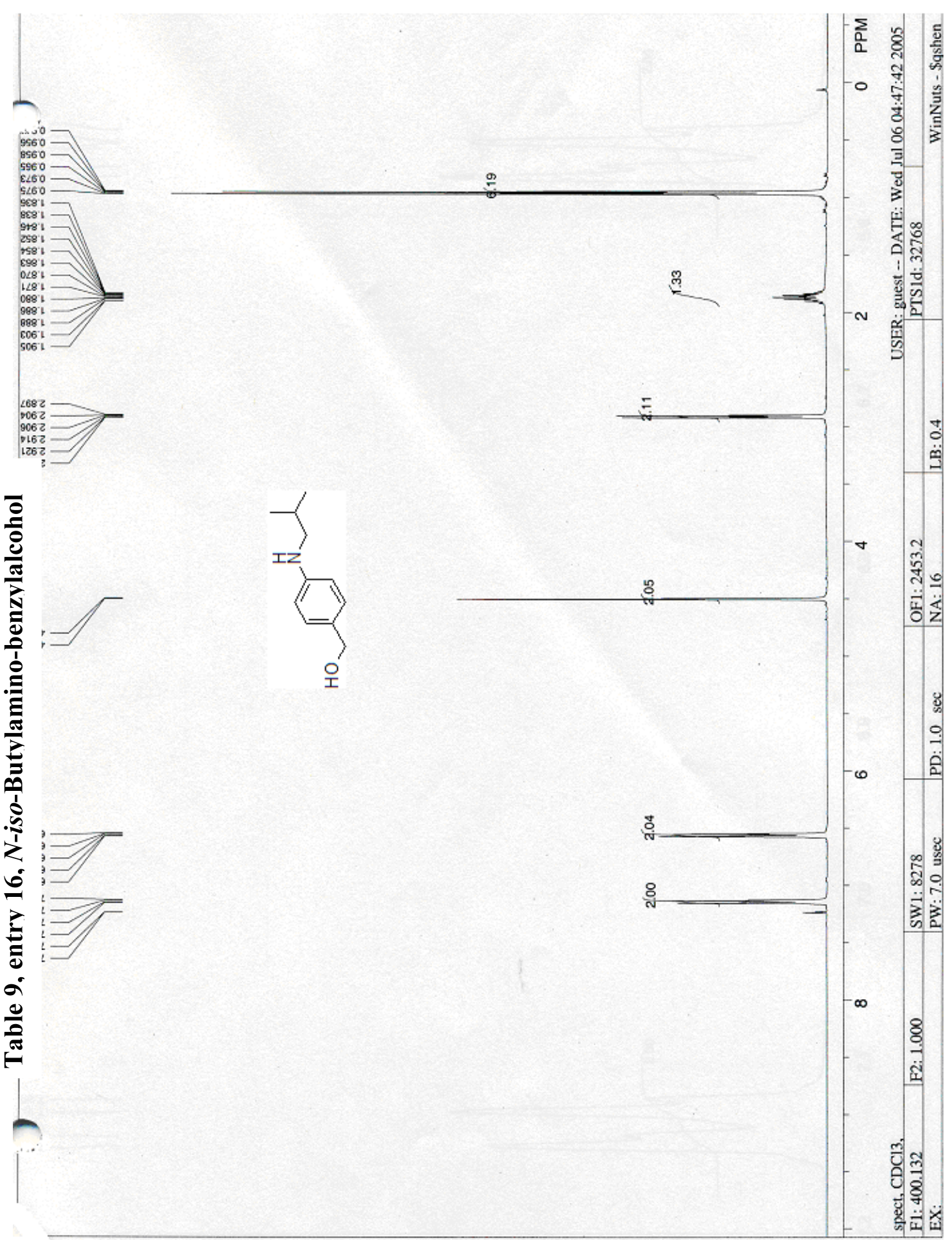
123

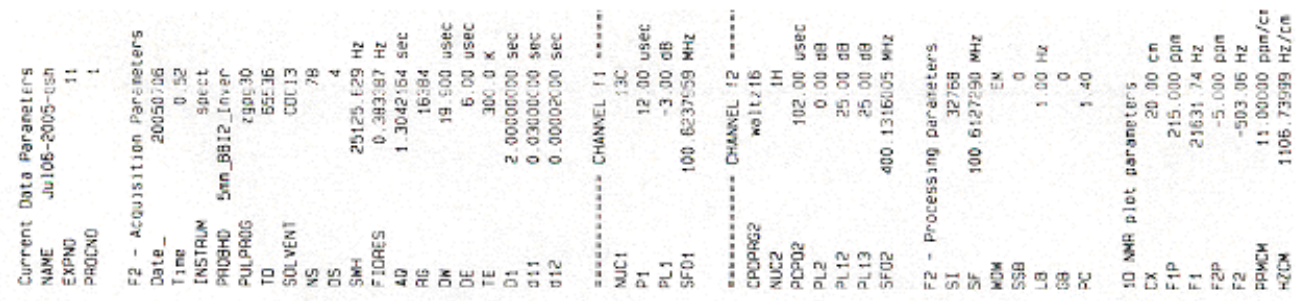



EEC' 29

OLB. 99

$D D I C L$ $08 L^{\circ} \angle L$

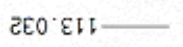

$8 \angle 2 \cdot 62$ $69 L$ ' 62

$9 L \angle B+L$

wdd

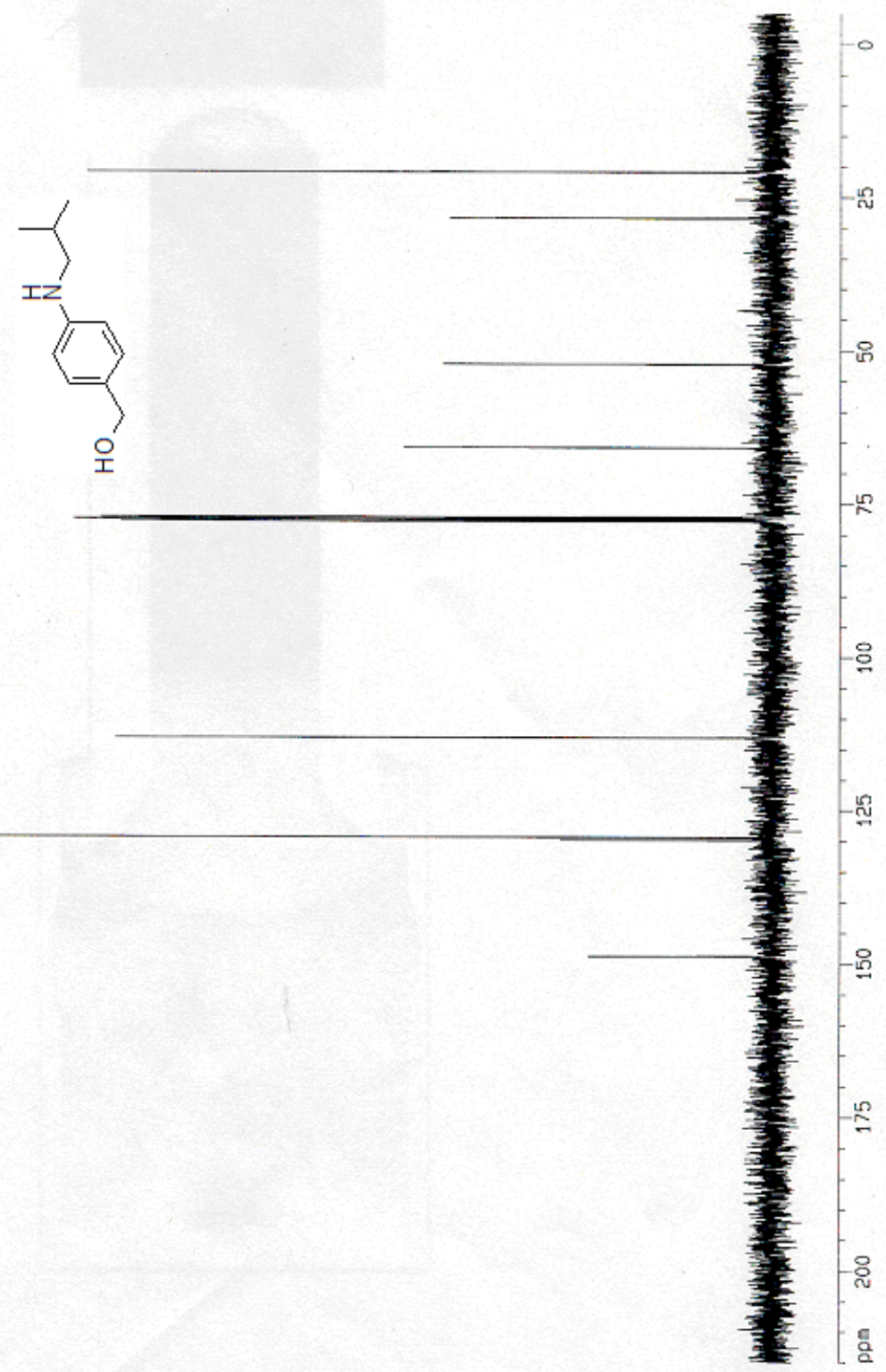




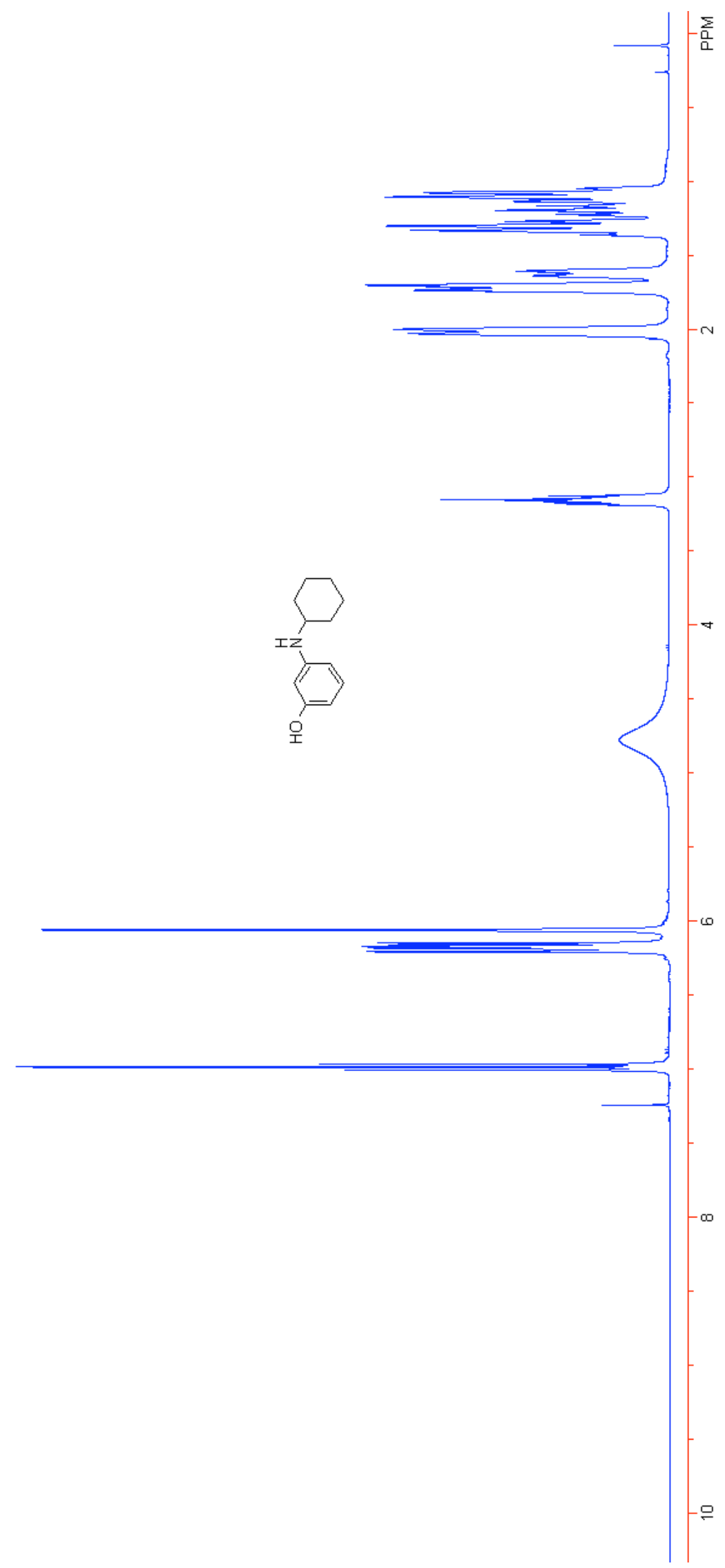

ט. 


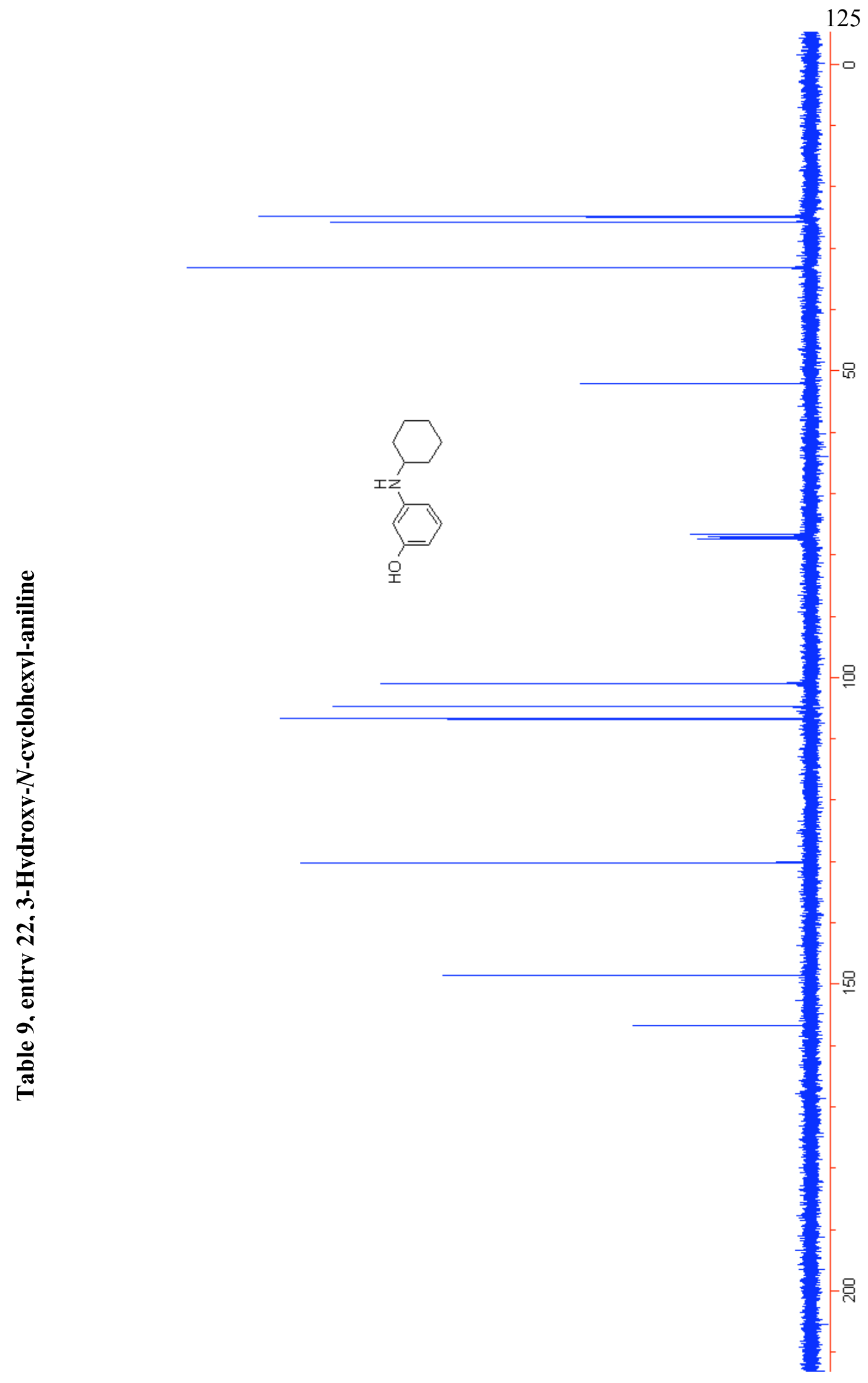




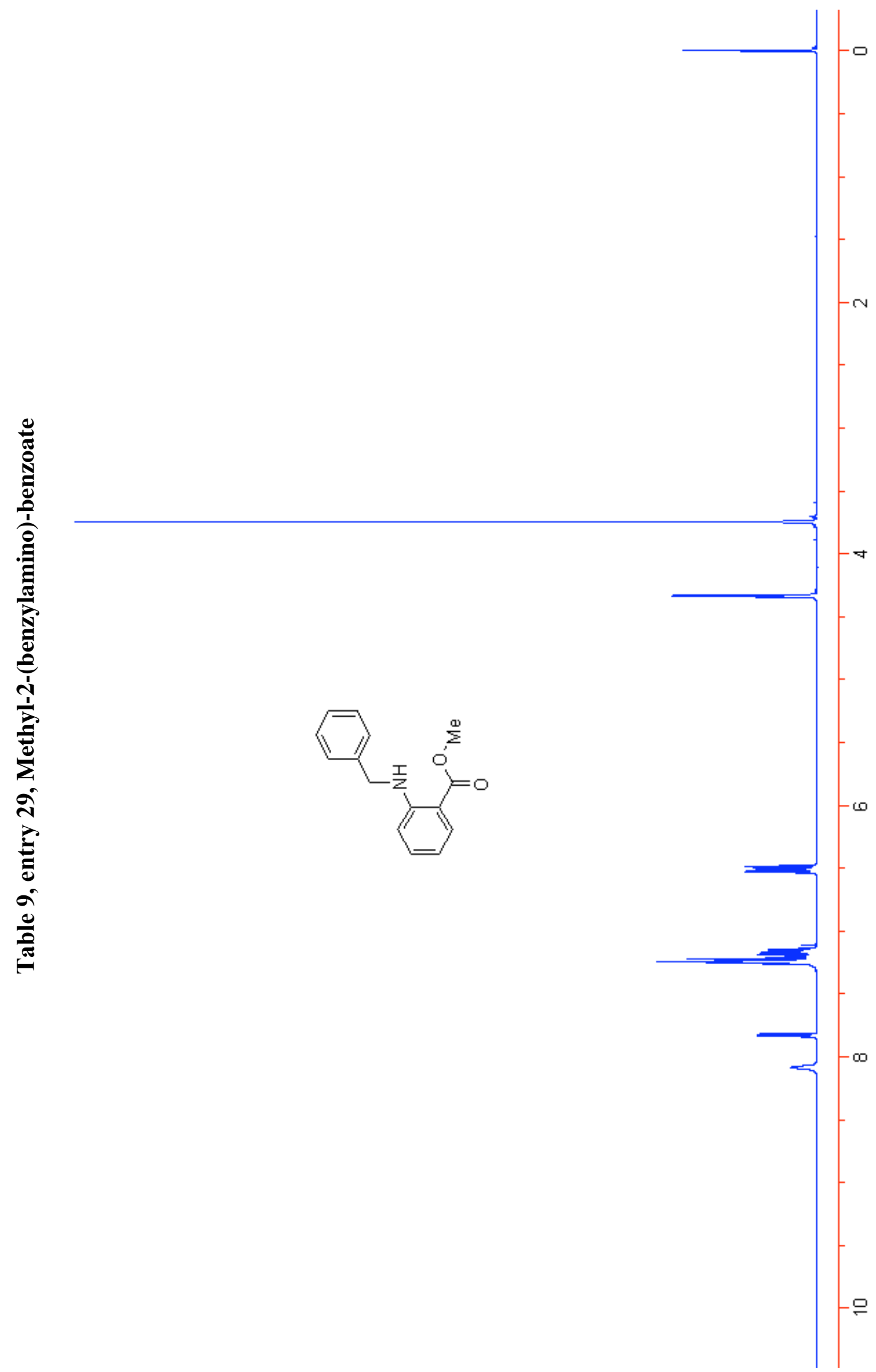



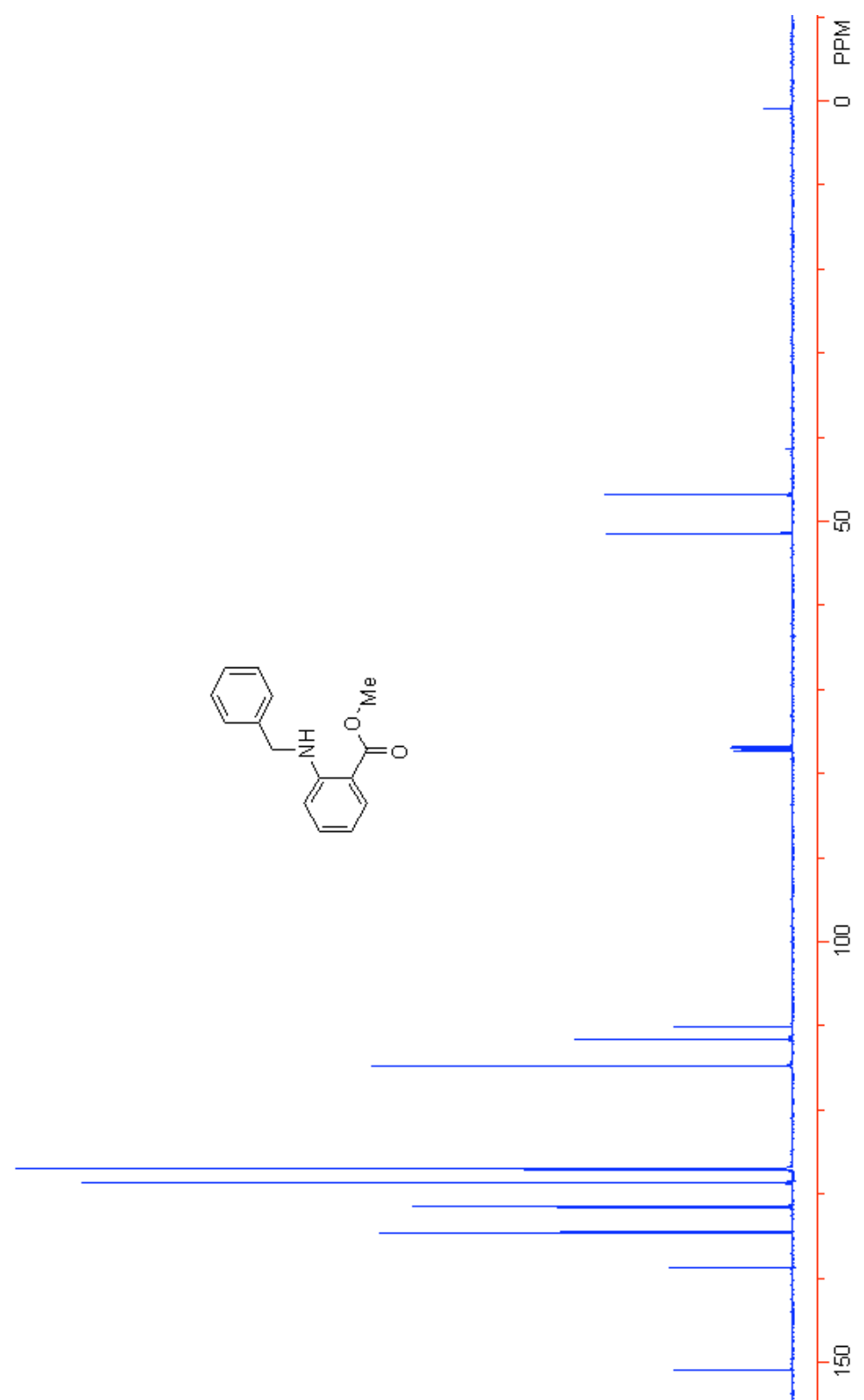


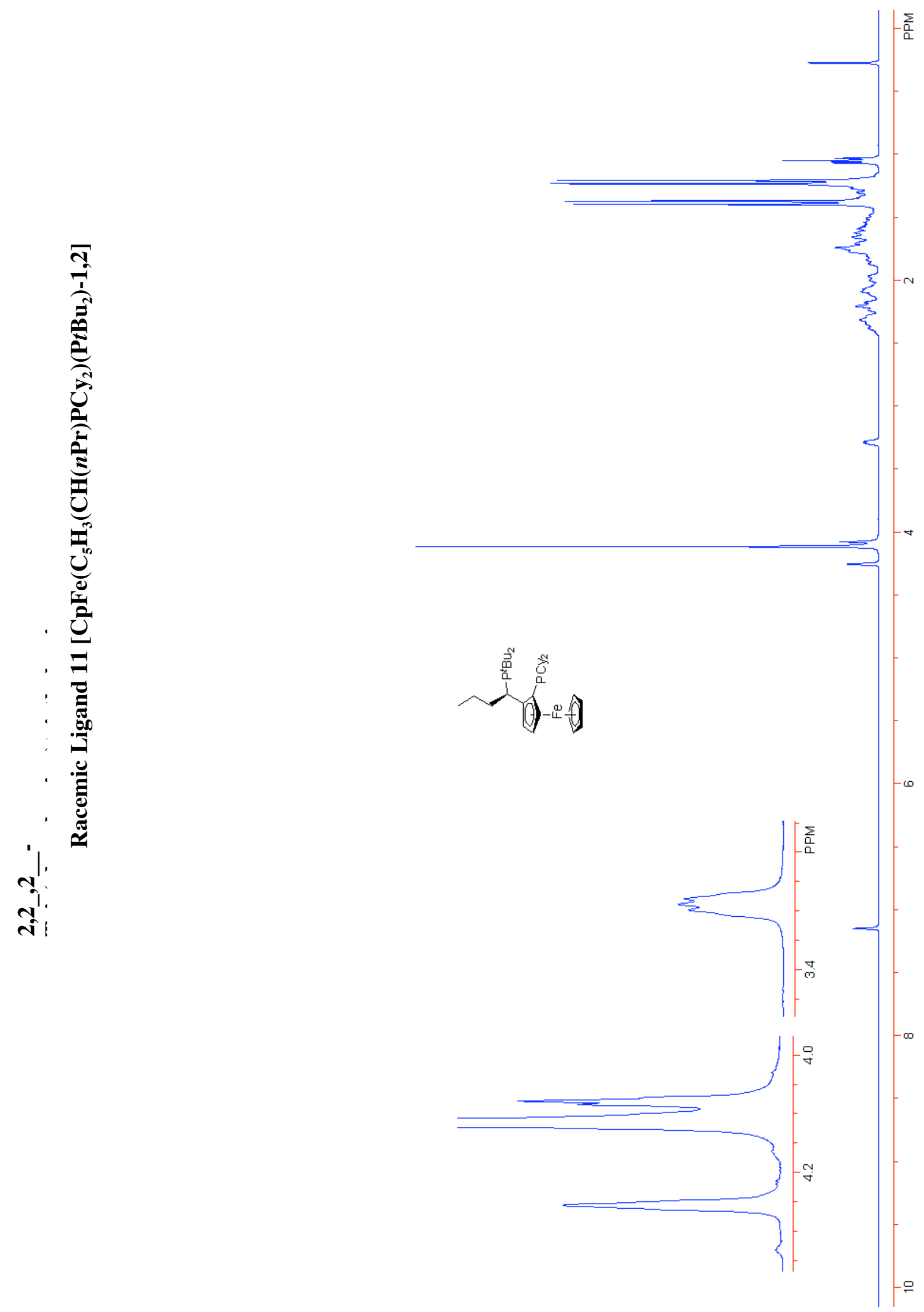



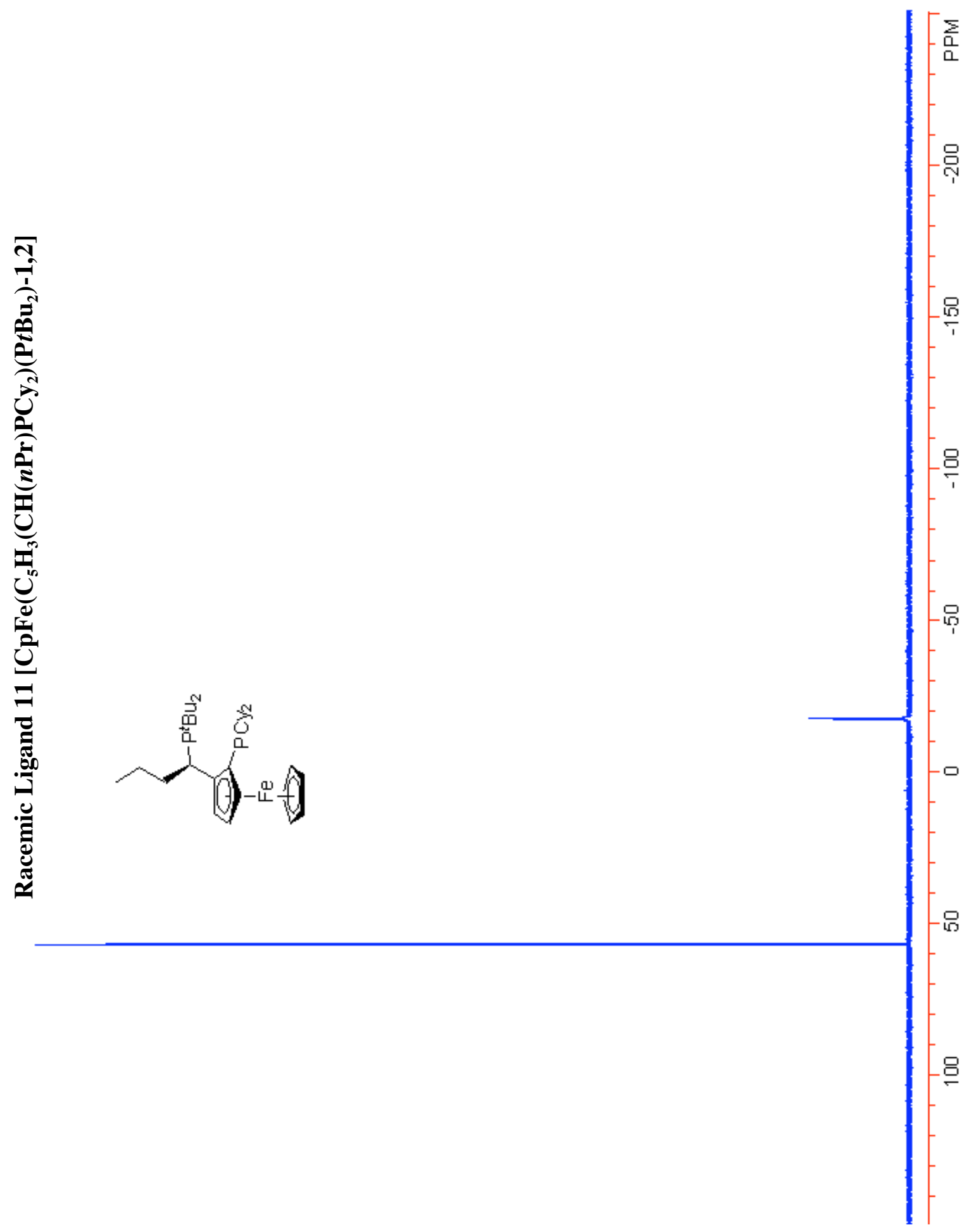


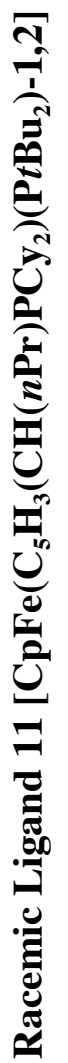
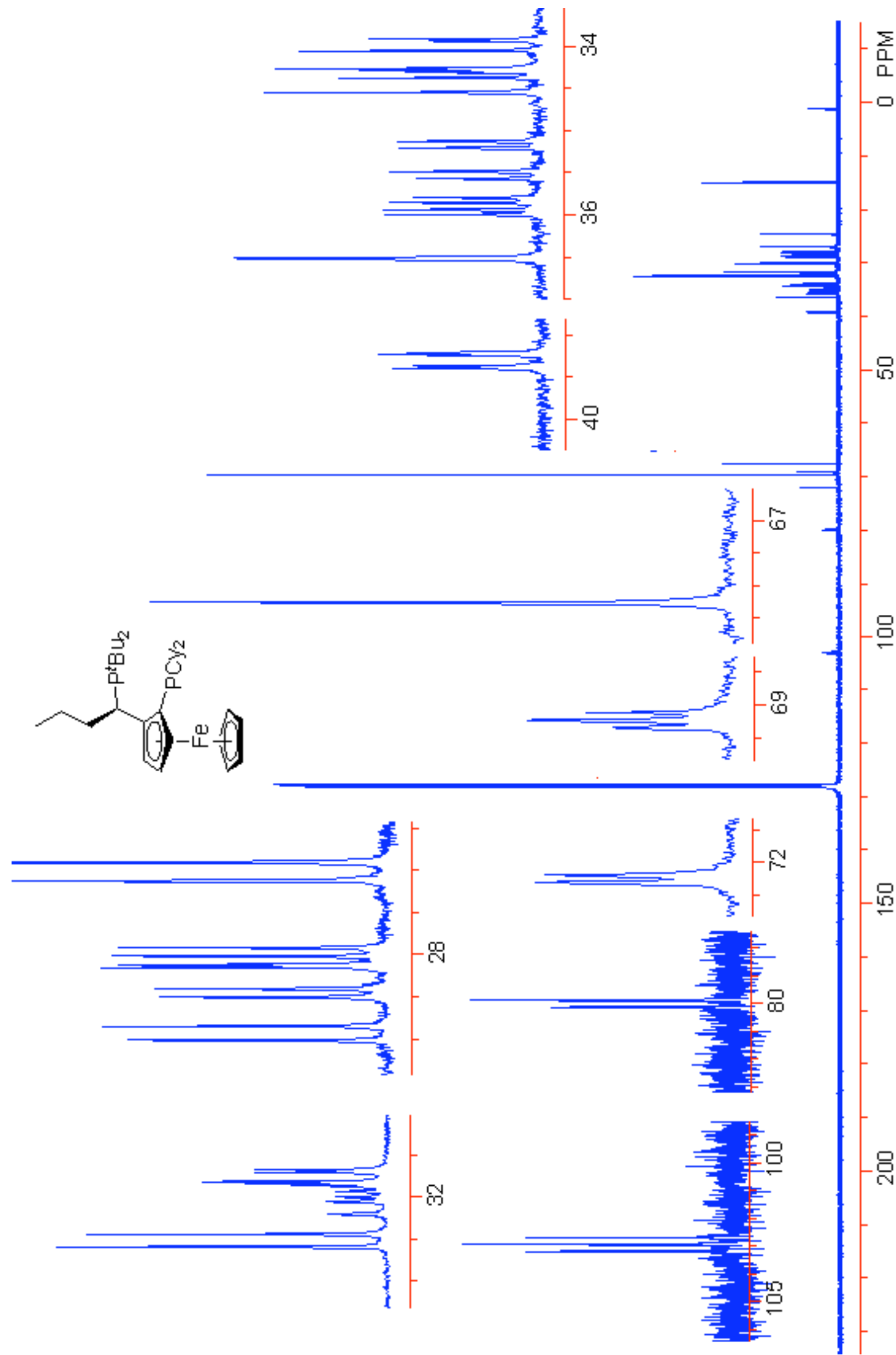

-啚 


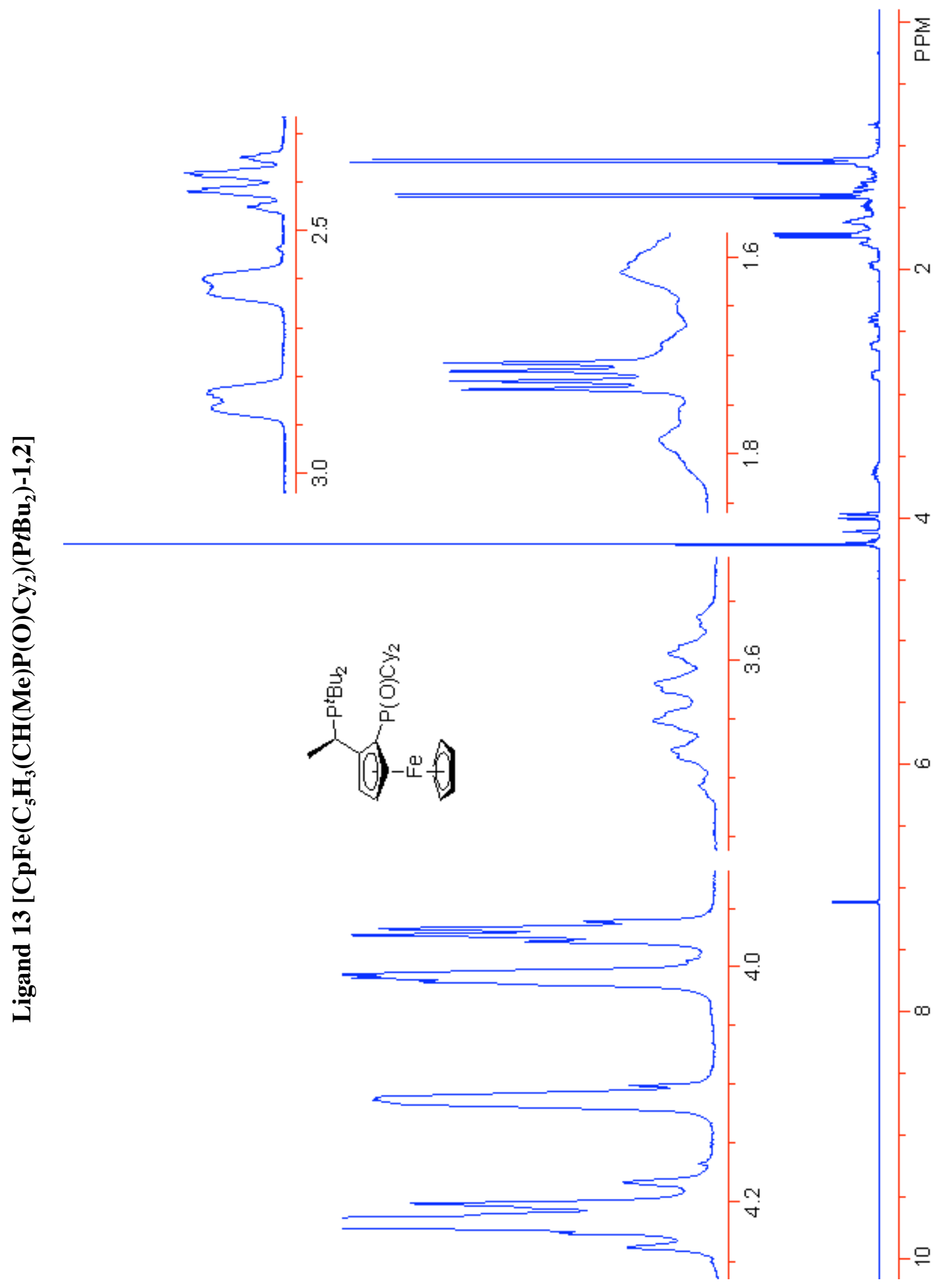




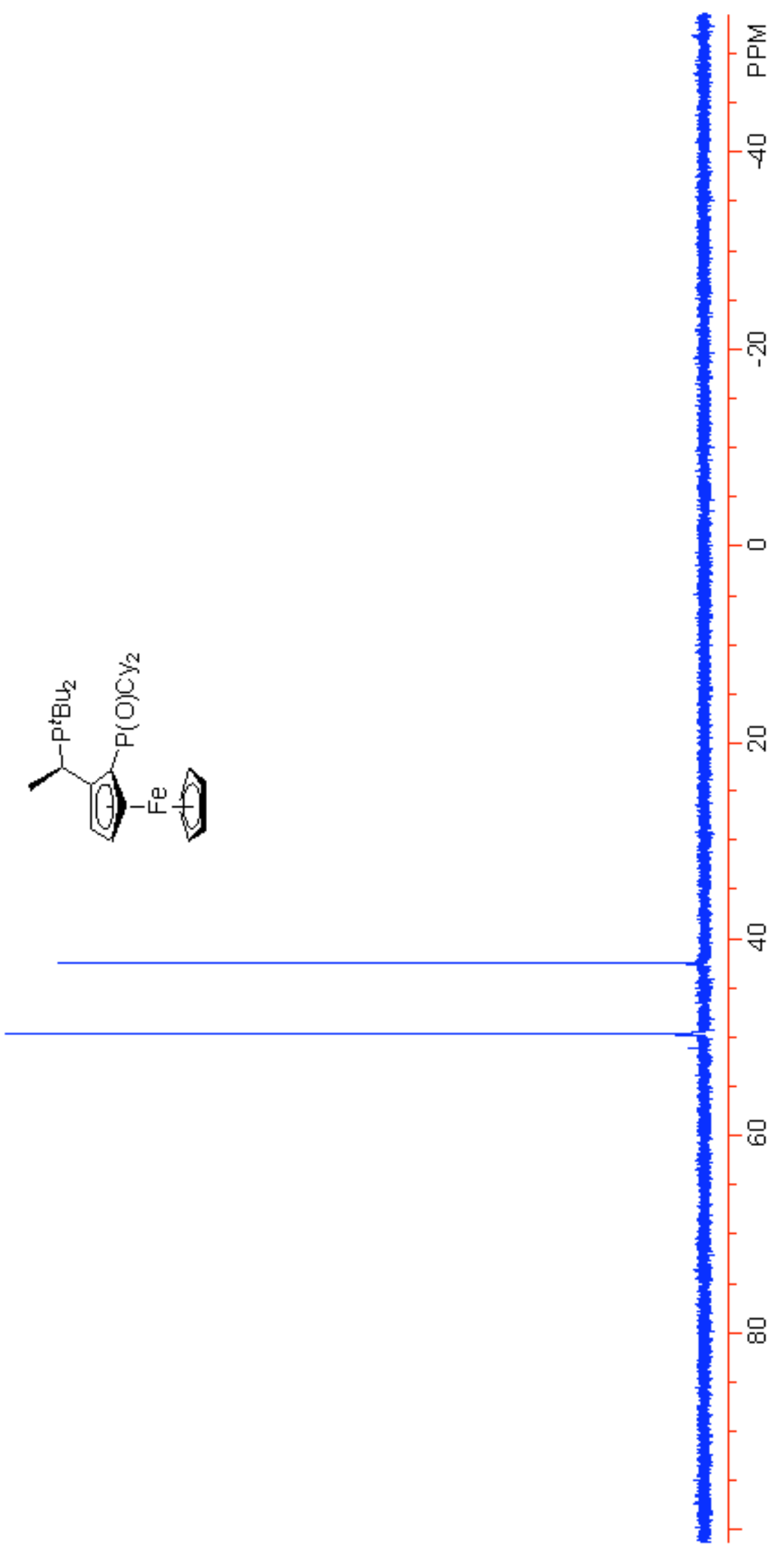




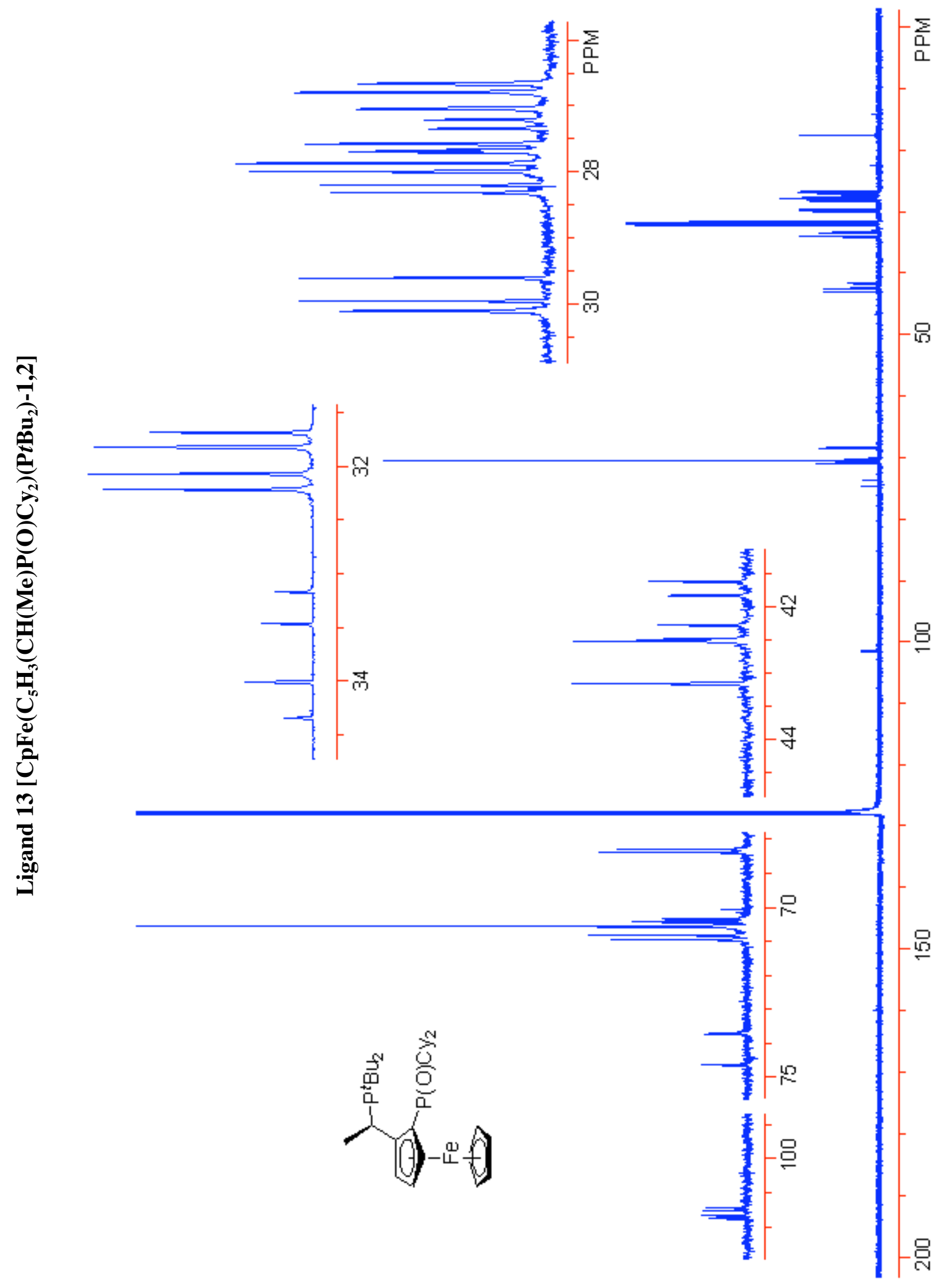




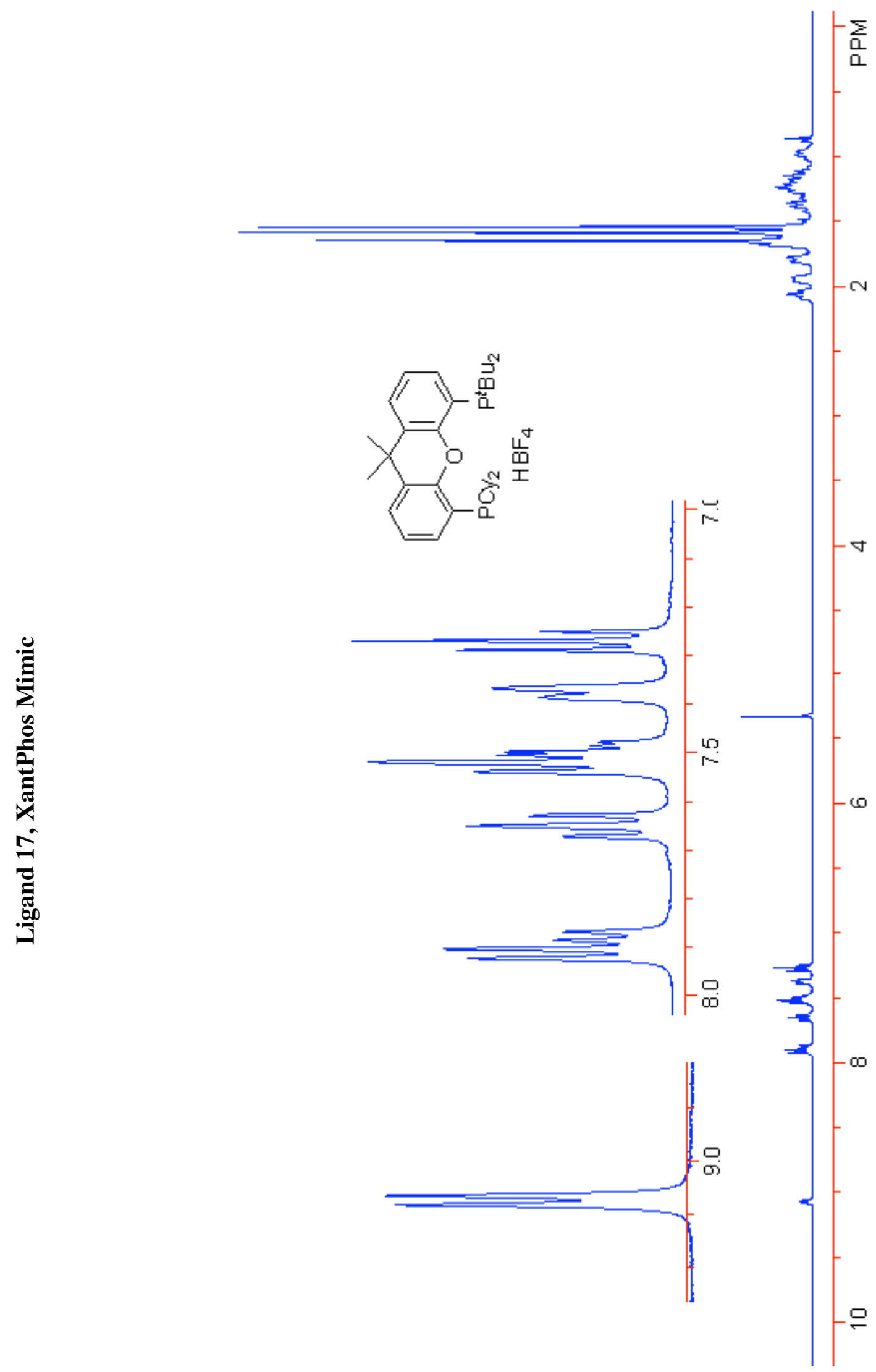


135

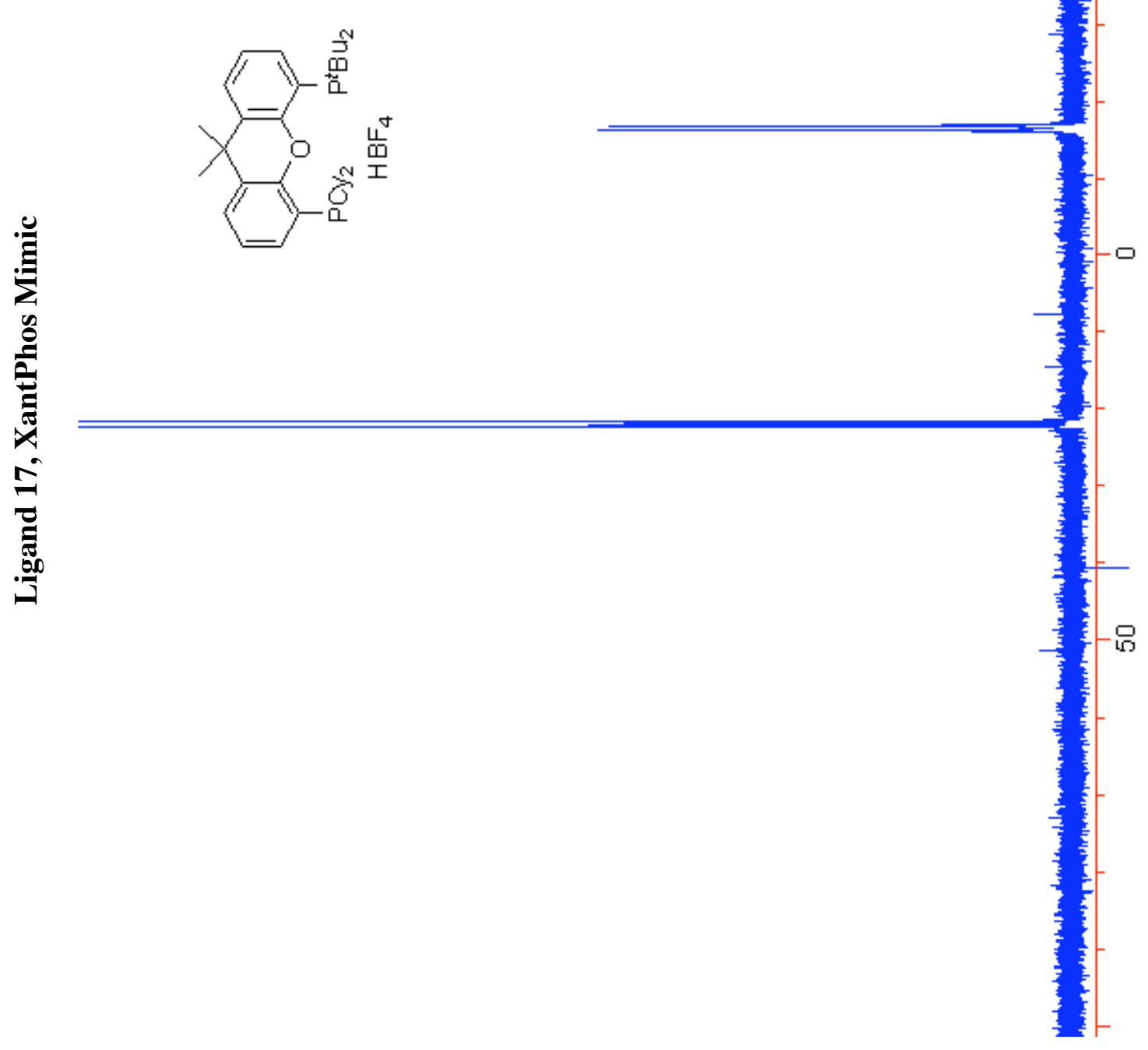




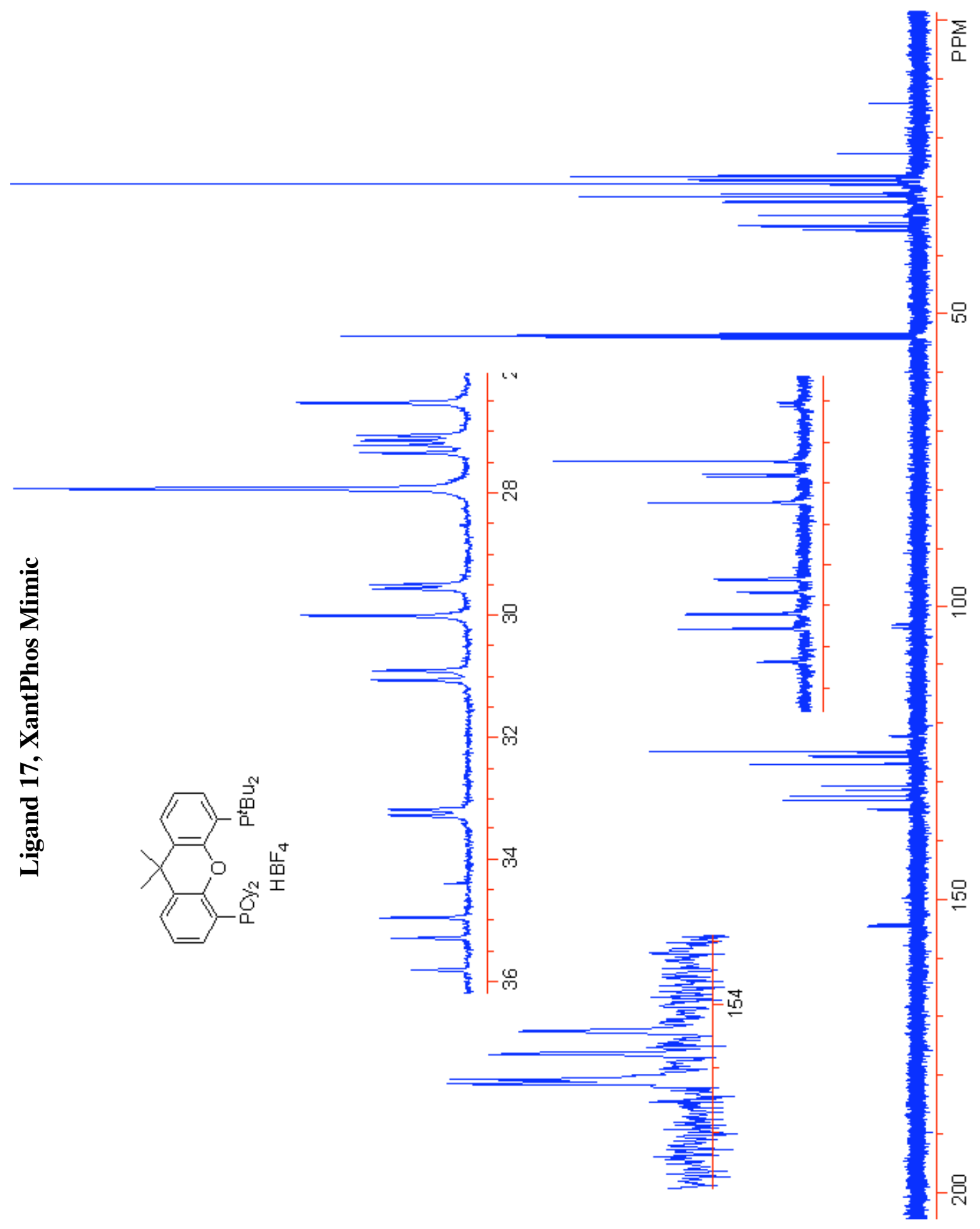

